\title{
Spatially Indirect Excitons in Coupled Quantum Wells
}

\author{
by \\ Chih-Wei Eddy Lai \\ B. S. (National Taiwan University) 1996
}

\begin{abstract}
A thesis submitted in partial fulfillment of the requirements for the degree of Doctor of Philosophy

in

Applied Science and Technology

in the

GRADUATE DIVISION

of the

UNIVERSITY OF CALIFORNIA, BERKELEY
\end{abstract}

\author{
Committee in charge: \\ Professor Daniel S. Chemla, Co-chair \\ Professor Ture K. Gustafson, Co-chair \\ Professor Steven G. Louie \\ Professor Peter Y. Yu
}

Spring 2004 
The dissertation of Chih-Wei Eddy Lai is approved:

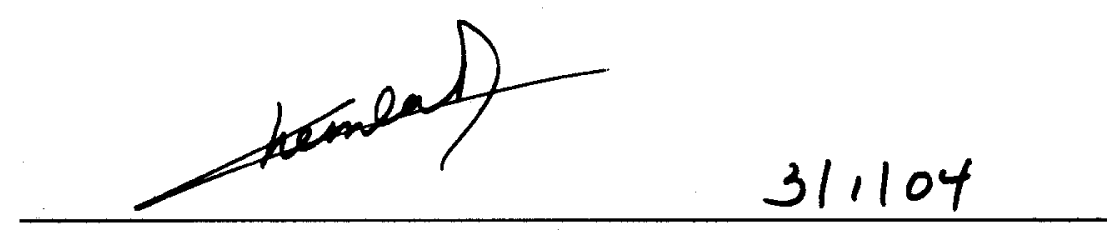

Chair

Date

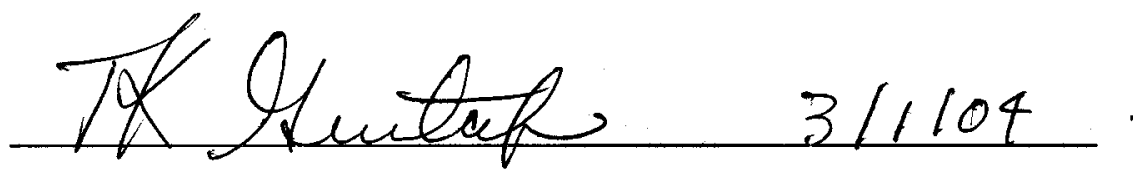

Co-chair

Date

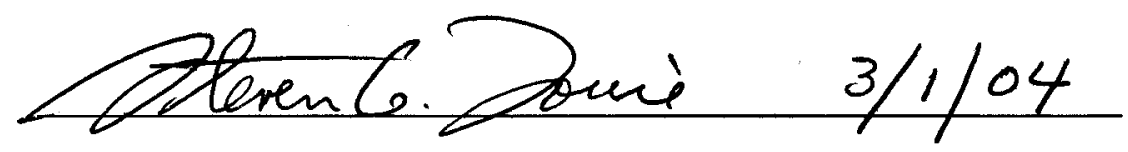

Date

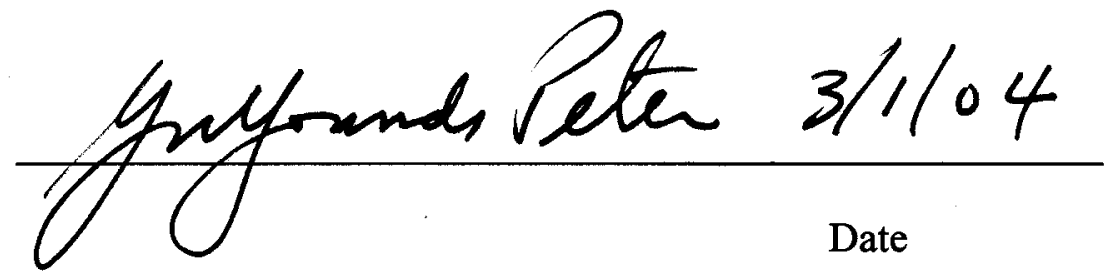

University of California, Berkeley

Spring 2004 


\author{
Abstract \\ Spatially Indirect Excitons in Coupled Quantum Wells \\ by Chih-Wei Eddy Lai \\ Doctor of Philosophy in Applied Science and Technology \\ University of California at Berkeley \\ Professor Daniel S. Chemla, Co-chair \\ Professor Ture K. Gustafson, Co-chair
}

Microscopic quantum phenomena such as interference or phase coherence between different quantum states are rarely manifest in macroscopic systems due to a lack of significant correlation between different states. An exciton system is one candidate for observation of possible quantum collective effects. In the dilute limit, excitons in semiconductors behave as bosons and are expected to undergo Bose-Einstein condensation (BEC) at a temperature several orders of magnitude higher than for atomic BEC because of their light mass. Furthermore, well-developed modern semiconductor technologies offer flexible manipulations of an exciton system. Realization of BEC in solid-state systems can thus provide new opportunities for macroscopic quantum coherence research. In semiconductor coupled quantum wells (CQW) under a cross-well static electric field, excitons exist as separately confined electron-hole pairs. These spatially indirect excitons exhibit a radiative recombination time much longer than their thermal relaxation time - a unique feature in direct band gap semiconductor based structures. Their mutual repulsive dipole interaction further stabilizes the exciton system at low temperature and screens in-plane disorder more effectively. All these features 
make indirect excitons in CQW a promising system to search for quantum collective effects.

Properties of indirect excitons in CQW have been analyzed and investigated extensively. The experimental results based on time-integrated or time-resolved spatially resolved photoluminescence (PL) spectroscopy and imaging are reported in two categories. (i) Generic indirect exciton systems: general properties of indirect exciton such as the dependence of exciton energy and lifetime on electric fields and densities were examined. (ii) Quasi-two-dimensional confined exciton systems: highly statistically degenerate exciton systems containing more than tens of thousands of excitons within areas as small as $(10 \mu \mathrm{m})^{2}$ was observed. The spatial and energy distributions of optically active excitons were used as thermodynamic quantities to construct a phase diagram of the exciton system, demonstrating the existence of distinct phases.

Optical and electrical properties of the CQW sample were examined thoroughly to provide deeper understanding of the formation mechanisms of these cold exciton systems. These insights offer new strategies for producing cold exciton systems, which may lead to opportunities for the realization of BEC in solid-state systems.
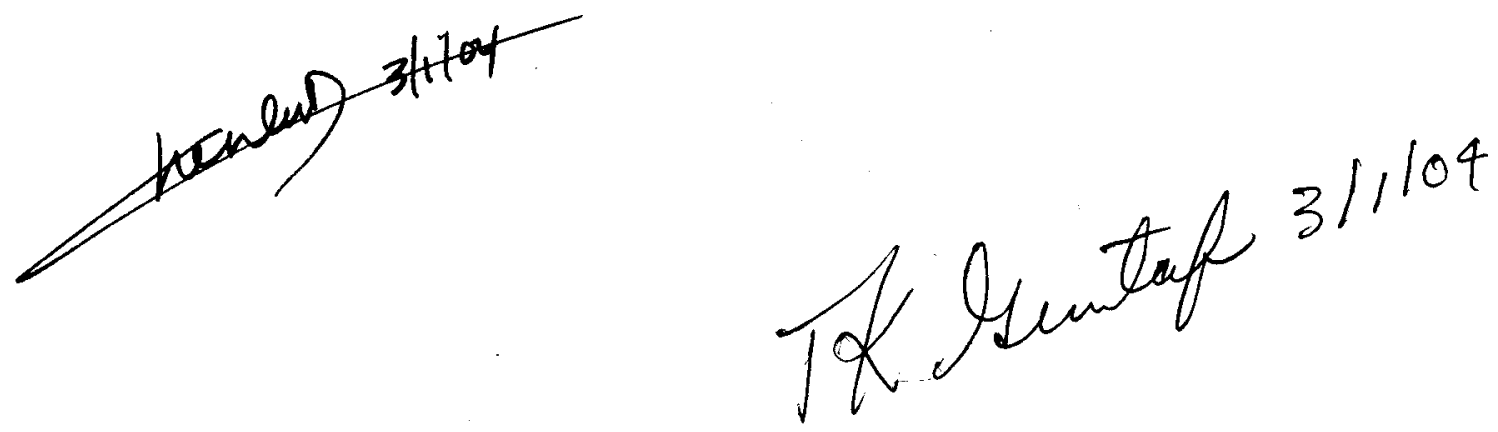
Spatially Indirect Excitons in Coupled Quantum Wells

Copyright 2004

By

Chih-Wei Eddy Lai 


\section{Table of Contents}

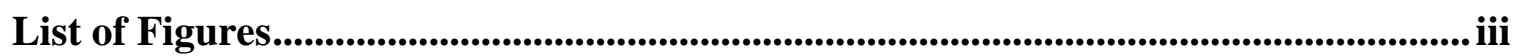

List of Tables .............................................................................................................................. v

Acknowledgments ....................................................................................................................... vi

Chapter 1 Introduction.................................................................................................. 1

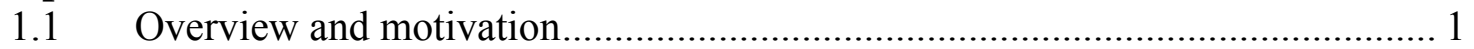

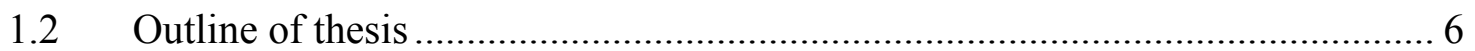

Chapter 2 Background ........................................................................................... 7

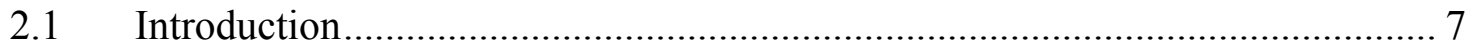

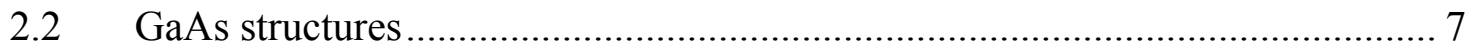

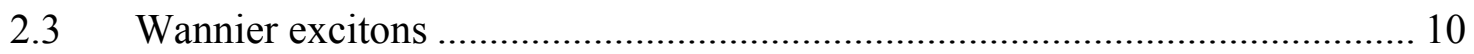

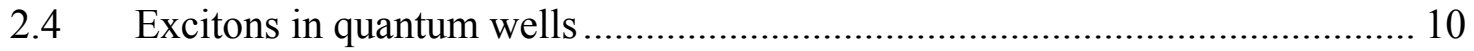

2.4.1 Electric and magnetic fields.............................................................. 11

2.5 Photoluminescence and life cycle of excitons ................................................ 14

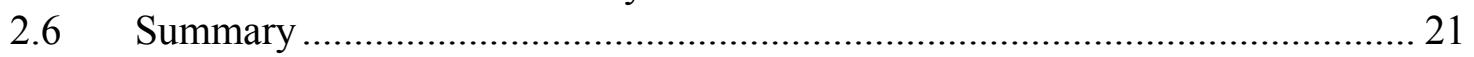

Chapter 3 Experimental Setup ................................................................................. 22

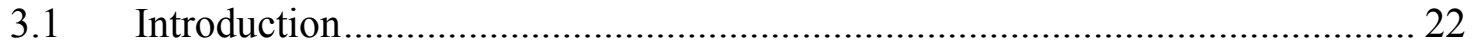

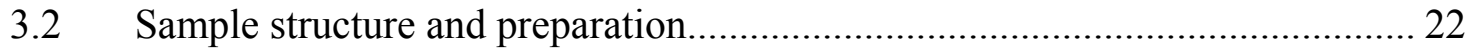

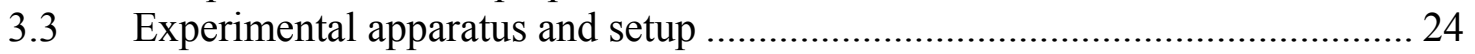

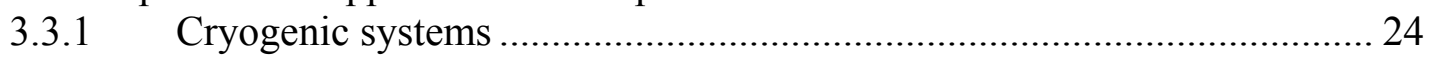

3.3.2 Laser systems and excitation sources …………..................................... 26

$3.4 \quad$ Optical properties: Photoluminescence......................................................... 27

3.4.1 Photoluminescence tomography and $\mu$-PL spectroscopy ......................... 29

3.4.2 Time-resolved photoluminescence …………………………………....... 30

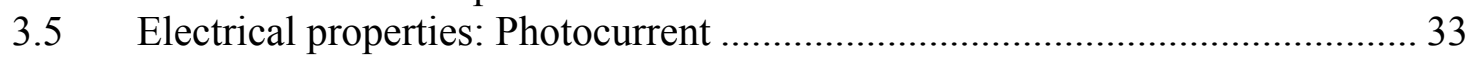

Chapter 4 Spatially Indirect Excitons ......................................................................... 35

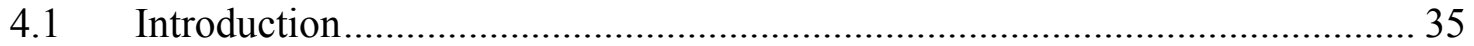

4.2 Exciton states in coupled quantum wells ........................................................ 35

4.3 Time-integrated photoluminescence spectroscopy …………............................ 37

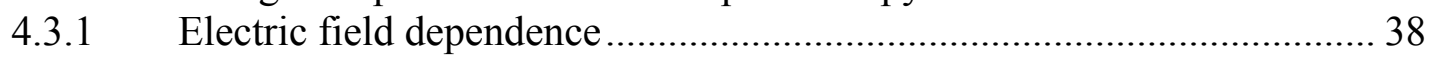

4.3.2 Excitation intensity dependence ……………...................................... 41

4.4 Time-resolved photoluminescence ……………............................................. 45

4.4.1 Spatially- and time-resolved photoluminescence ...................................... 47

4.4.2 Electric field dependence ..................................................................... 49

4.4.3 Excitation intensity dependence ……………….................................... 51

4.4.4 Excitation pulse duration dependence ................................................... 55

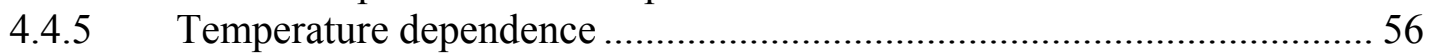

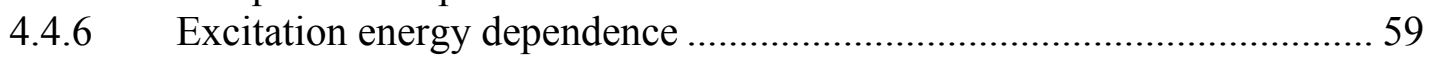

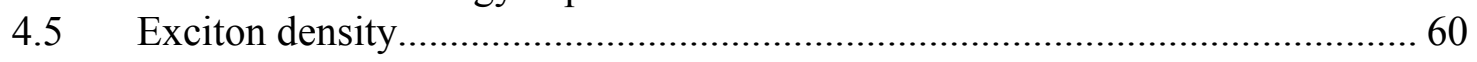

4.5.1 Mean field energy and exciton energy shift............................................. 60 


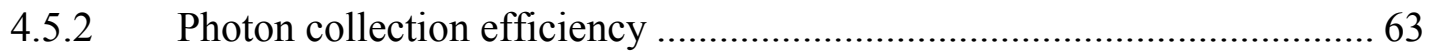

4.6 Effective mass and magneto-optical properties .................................................. 64

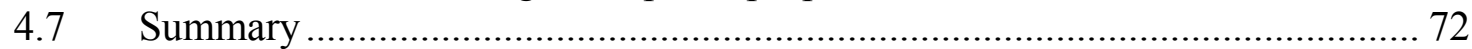

Chapter 5 Confined Exciton Systems............................................................................ 74

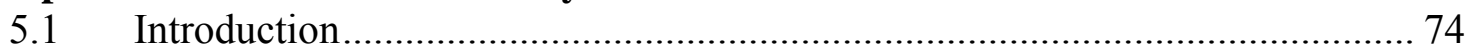

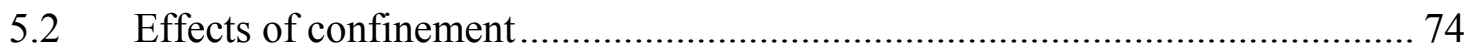

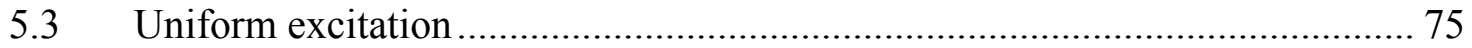

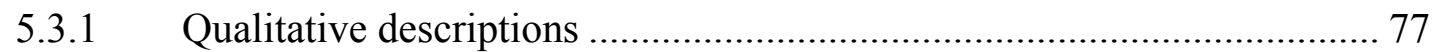

5.3.2 Phase diagram: $\mu$-PL imaging ............................................................ 79

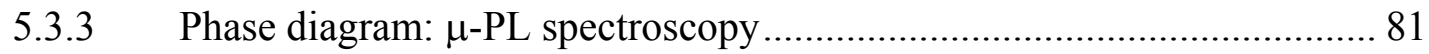

5.3.4 Comparison with remote homogeneous locations ..................................... 89

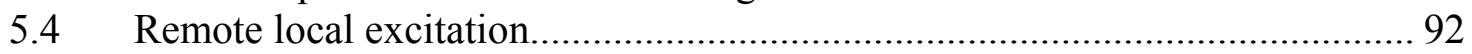

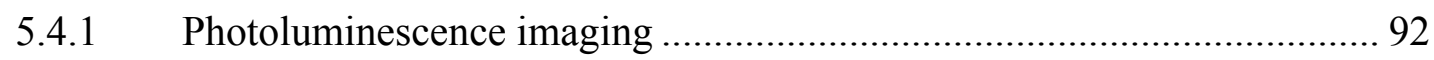

5.4.2 Photoluminescence spectroscopy...……………................................... 94

5.4.3 Time-resolved photoluminescence ………………................................. 95

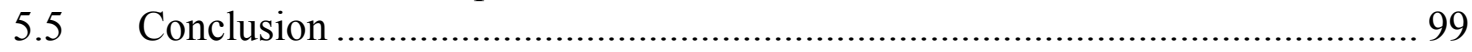

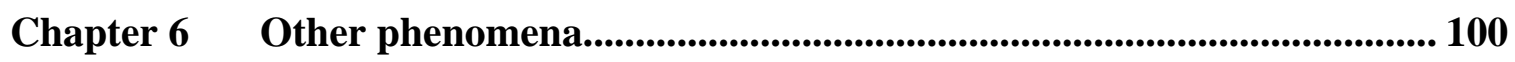

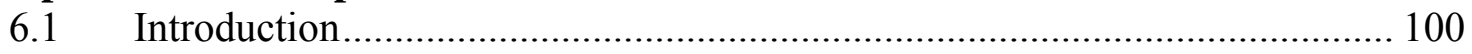

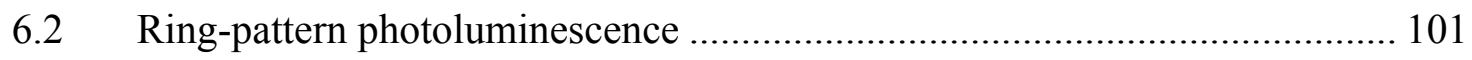

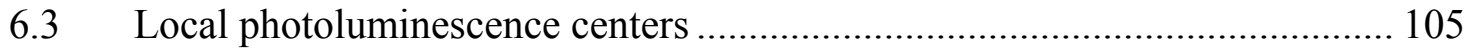

Chapter 7 Formation mechanisms .............................................................................. 107

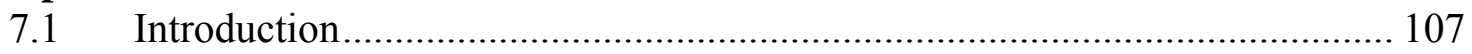

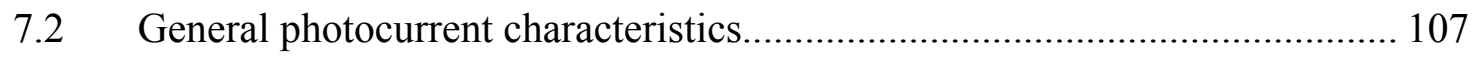

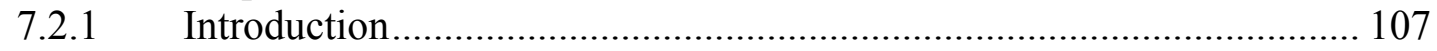

7.2.2 Stationary photocurrent measurement …………................................... 108

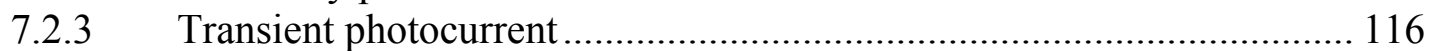

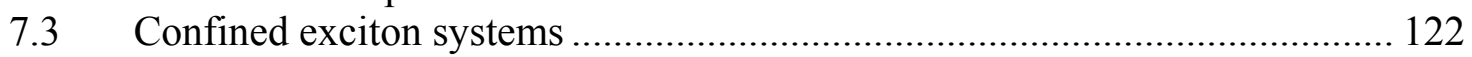

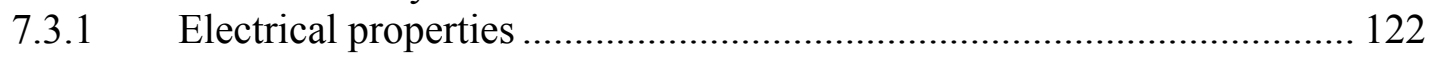

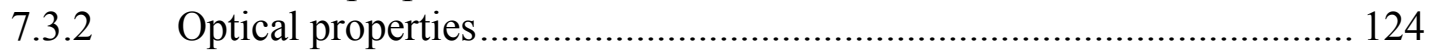

7.4 Photocurrents versus photoluminescence ……......................................... 126

7.4.1 General spatially indirect exciton systems............................................ 126

7.4.2 Confined spatially indirect exciton systems ……………….................... 135

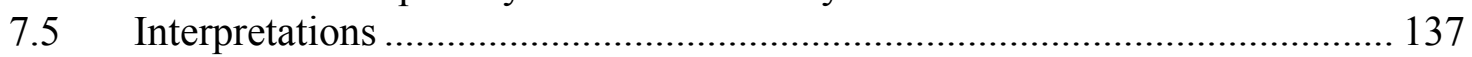

7.5.1 Confined exciton systems ……………………................................. 137

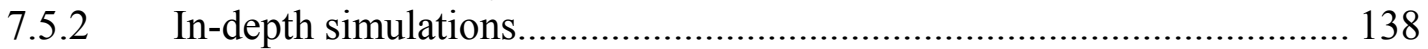

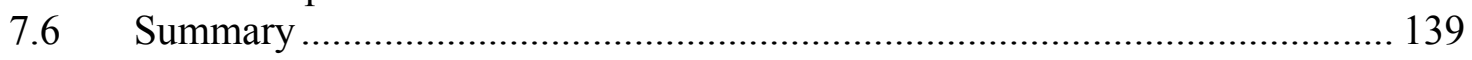

Chapter 8 Conclusion ......................................................................................................... 141

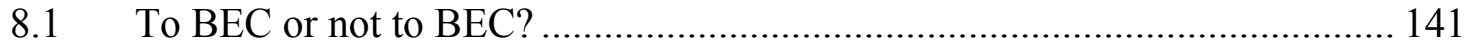

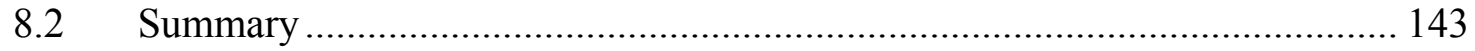

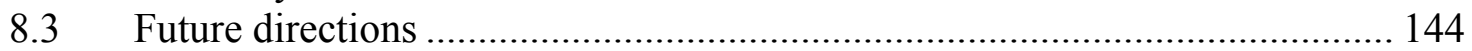

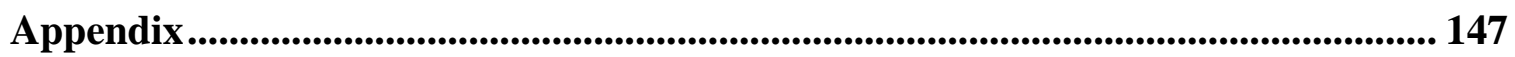

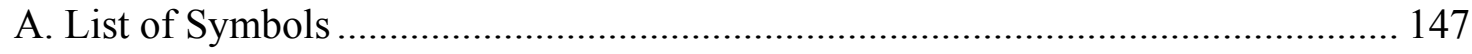

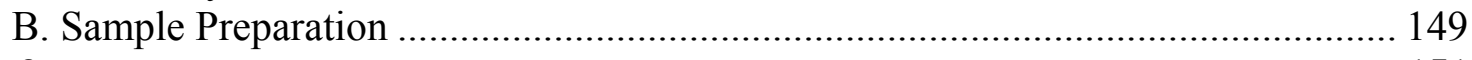

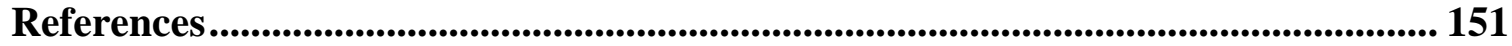




\section{List of Figures}

Number

Figure 1.1 Illustration of the electronic and excitonic phases in semiconductors. 3

Figure 2.1 Schematic diagram of energy levels and transitions in a CQW..................... 12

Figure 2.2 Energy-momentum dispersion and an example life cycle of excitons............ 15

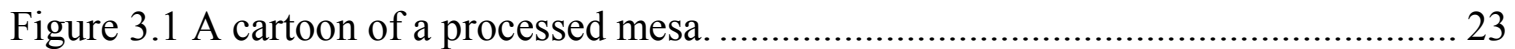

Figure 3.2 Schematic of a Janis Super VariTemp (SVT) cryostat.................................... 24

Figure 3.3 Spectral profile of the HeNe laser and laser diodes.. ……….......................... 27

Figure 3.4 Schematic of the $\mu$-PL imaging, spectroscopy, and PL dynamics setups. ...... 28

Figure 3.5 Indirect-X PL versus transmission of a band pass interference filter.............. 30

Figure 3.6 Block diagram of the photoluminescence dynamics setup.............................. 32

Figure 3.7 Electronic connections of the photocurrent transient measurements. .............. 34

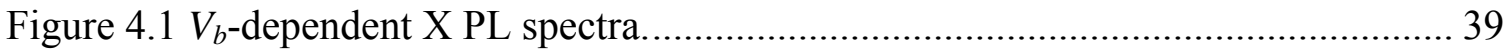

Figure 4.2 HeNe excitation intensity dependent PL spectra at $V_{b}=-0.95 \mathrm{~V}$.................... 42

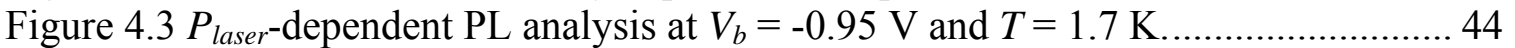

Figure 4.4 $P_{\text {laser }}$-dependent exciton PL energy and peak intensity................................. 45

Figure 4.5 Spatially-resolved PL spectra and time-resolved PL..................................... 47

Figure 4.6 $V_{b}$-dependent PL dynamics of indirect-Xs .................................................. 50

Figure $4.7 V_{b}$-dependent PL decay time of indirect-Xs. ............................................... 51

Figure 4.8 $P_{\text {laser }}$-dependent PL dynamics...................................................................... 52

Figure 4.9 $P_{\text {laser }}$-dependent X PL decay/rise time under $V_{b}=-0.95 \mathrm{~V}$ and $-1.5 \mathrm{~V}$........... 53

Figure $4.10 P_{\text {laser }}$-dependent PL spectra (normalized) at $V_{b}=-0.95 \mathrm{~V}$............................ 54

Figure 4.11 TRPL versus the excitation pulse duration................................................. 55

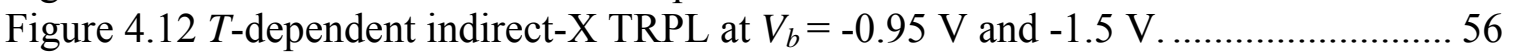

Figure $4.13 T$-dependent indirect-X PL decay time, peak energy, and linewidth. ........... 57

Figure 4.14 Estimated exciton density versus $P_{\text {laser }}$ at $V_{b}=-0.95 \mathrm{~V}$................................. 62

Figure 4.15 2D-magnetoexciton center-of-mass and internal motions coupling.............. 68

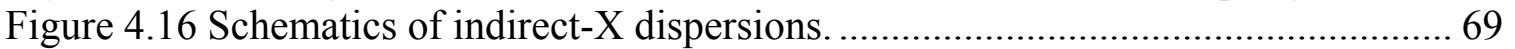

Figure 4.17 Magnetoexciton dispersions and magnetoexciton effective mass................. 70

Figure 5.1 CCD PL image of a $780 \mu \mathrm{m}$ mesa with two natural 'traps'........................... 76

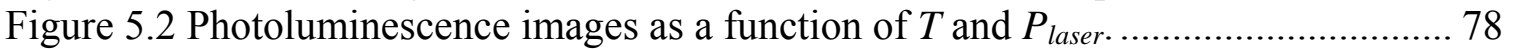

Figure 5.3 Spatially-resolved PL spectra across a 'trap' ................................................. 78

Figure 5.4 X-cloud radius $\left(\sigma_{\mathrm{PL}}\right)$ phase diagram of the confined indirect-X systems ....... 80

Figure 5.5 Kurtosis $\left(\mathrm{K}_{\mathrm{PL}}\right)$ phase diagram of the confined indirect-X systems ................. 80

Figure 5.6 Spatially-resolved PL spectra of the confined indirect-X systems.................. 82

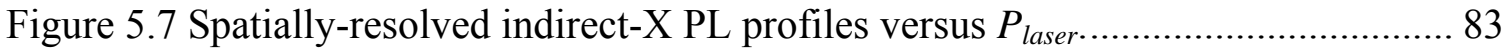

Figure 5.8 Spatially-resolved PL spectra analysis versus T.......................................... 86 
Figure 5.9 Analysis of $T$-dependent PL spectra of the confined indirect-X systems.. ..... 88

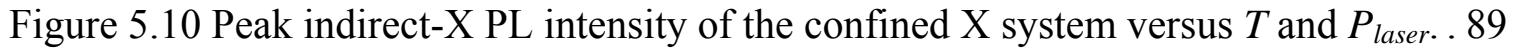
Figure 5.11 Indirect-X PL spectra at the 'trap' center (TC) versus remote spots (RS)... 90 Figure 5.12 T-dependent indirect-X peak and average energies: TC versus RS. ............. 91 Figure 5.13 T-dependent indirect-X PL linewidth at remote spots................................. 91 Figure 5.14 PL images of confined indirect- $X$ systems under a remote local excitation.. 93 Figure 5.15 False color 3D PL images around Trap-B................................................. 93 Figure 5.16 Integrated PL under a uniform and remote localized HeNe excitation......... 94 Figure $5.17 V_{b}$-dependent PL spectra and TRPL of Trap-A............................................ 96

Figure 5.18 TRPL of indirect-Xs from Trap-A versus $\Delta \mathrm{y}$ at $V_{b}=-0.95 \mathrm{~V}$....................... 97

Figure $5.19 P_{\text {laser }}$-dependent TRPL of indirect-Xs from Trap-A at $V_{b}=-0.95 \mathrm{~V}$............. 97 Figure 5.20 T-dependent TRPL of indirect-Xs from Trap-A at $V_{b}=-0.95$ and $-1.5 \mathrm{~V}$.... 98 Figure 5.21 Spatially resolved TRPL of indirect-Xs from Trap-A at $V_{b}=-0.95 \mathrm{~V}$.......... 98

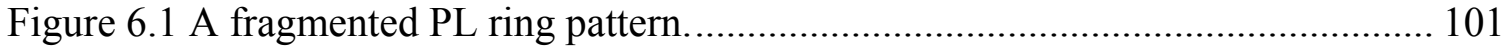

Figure 6.2 Spatially resolved PL spectra of PL rings ………....................................... 103

Figure 6.3 $P_{\text {laser }}$-dependent indirect-X PL intensity and energy of PL rings. ................. 103

Figure $6.4 V_{b}$-dependent PL images at $T=1.7 \mathrm{~K}$ under a uniform HeNe excitation ..... 106

Figure 7.1 Current-voltage characteristics in the dark.............................................. 108

Figure 7.2 Photocurrent-voltage characteristics under a local HeNe excitation............. 109

Figure 7.3 Zoom-in view near the origin of the photocurrent-voltage characteristics. .. 110

Figure 7.4 $P_{\text {laser }}$-dependent short-circuit current......................................................... 111

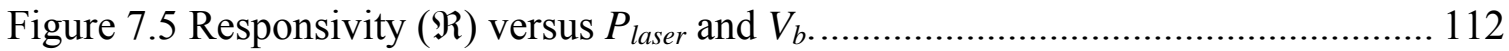

Figure 7.6 Transient photocurrent at $V_{b}=-1$ and $+1 \mathrm{~V}$.............................................. 117

Figure 7.7 Transient photocurrent (TPC) measured with different pulse durations....... 118

Figure 7.8 Time-resolved I-V characteristics .......................................................... 119

Figure 7.9 Transient photocurrent versus the laser repetition rate................................. 120

Figure 7.10 Photocurrent versus laser excitation locations. ........................................... 123

Figure 7.11 I-V characteristics under a local HeNe excitation near traps. ..................... 123

Figure 7.12 Spatially-resolved PL spectra from Trap-A at $V_{b}=0 \ldots \ldots \ldots \ldots \ldots \ldots \ldots \ldots \ldots \ldots . . .125$

Figure 7.13 Photocurrents versus estimated $X$ photons................................................. 128

Figure $7.14 V_{b}$-dependent integrated PL: HeNe vs. laser diode $(\mathrm{h} v=1.802 \mathrm{eV}) \ldots \ldots \ldots \ldots . .132$

Figure $7.15 V_{b}$-dependent PL spectra: HeNe vs. laser diode $(\mathrm{h} v=1.802 \mathrm{eV}) \ldots \ldots \ldots \ldots \ldots . .133$

Figure 7.16 PL- and I-V characteristics: HeNe vs. laser diode $(\mathrm{h} v=1.802 \mathrm{eV}) \ldots \ldots \ldots \ldots . .135$

Figure 7.17 TRPL of indirect-Xs from Trap-A versus the transient photocurrent ......... 136 


\section{List of Tables}

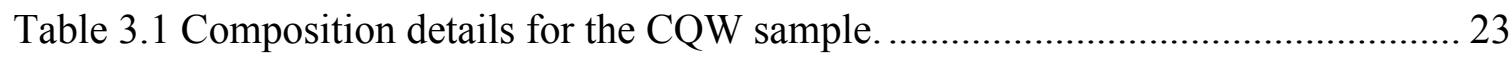

Table 3.2 Photoluminescence dynamics equipment list. ................................................... 33

Table 4.1 List of symbols characterizing exciton photoluminescence. ............................ 38

Table 6.1 List of measurements and experimental parameters....................................... 100 


\section{Acknowledgments}

I wish to express my deep gratitude to my advisor, Daniel Chemla, for his guidance, encouragement, support, and willingness to entrust so much of the development of the project to my judgment. I am also grateful to Ken Gustafson and Peter Yu's help and advice during the dissertation writing process.

In my first couple years in Chemla's group, Neil Fromer taught me many cryogenic and experimental skills such as how to do a four-wave mixing experiment. In the beginning of 2001, I began working on the indirect exciton project introduced by Leonid Butov. I built another experimental setup and took independent research after 2002. These two years of independent research has been challenging and rewarding. Through these years, I have a great time with a hard-working intelligent crew: Marc Carnahan, Robert Kaindl, Jean Zoch, Daniel Haegele, Andreas Schumacher, Keshave Dani, Michael Briet, Jerome Tignon - many of whom were there to offer advices or help with my various projects. And without the professional technical support from people like Bruce Rude, John Pepper and many others, my graduate research would not have progressed smoothly.

The company of Marc, Neil, Nuh Gedik, Cristopher Webber, Keshave made my long days of data-taking more enjoyable. I am very fortunate to have many close friends from Taiwan (Sherry Cho, Chih-Wei Chang, Yun-pei Chang, Da-Wei Chiou, Chih-hao $\mathrm{Li}$, and many others). The games, parties, and hiking activities with them provided much 
needed play-time. The regular weekly lunch debates on various physics subjects with them made studying physics fun and kept me upbeat during the most difficult years in my life when I almost gave up being a scientist.

I am grateful to the support of my family over the years, especially my parents' patience and love. Finally, I am most thankful for the sun that has shone in my own life throughout these years because of the constant love and support of my fiancée, Chen-hui. Our physical separation across the Pacific Ocean or the North American Continent will have to continue across the Atlantic Ocean. But, with a high binding energy, we will remain a boud pair as always.

Berkeley, California Chih-Wei Lai

February, 2004 



\section{Chapter 1 Introduction}

\subsection{Overview and motivation}

Microscopic quantum phenomena such as interference or phase coherence between different quantum states are rarely manifest in macroscopic systems due to a lack of significant correlation between different states. Several phenomena, such as superconductivity, superfluidity, laser action, Bose-Einstein condensation (BEC), and quantum Hall effects which involve superpositions of states of bosons or fermions at macroscopic scales, are striking examples of macroscopic quantum coherent states. Among these, Bose-Einstein condensation probably is most elusive. The existence of BEC was first predicted by Einstein based on Bose statistics as a new form of matter in 1924-25 [1-3]. BEC was inferred in superfluid liquid helium [4] but a more pure BEC was not created until almost 70 years later with ultra-cold $(\sim 100 \mathrm{nK}$ to $1 \mu \mathrm{K})$ dilute gases of composite-boson alkali atoms in magneto-optical traps $[5,6]$. Over the past few years, the subsequent demonstration of BEC in several atomic species confined in optical or magnetic traps has triggered intense interest [7-10].

A variety of quasi-particles with bosonic character are also found in condensed matter. In particular, semiconductors can sustain bound electron-hole (e-h) pairs, excitons (Xs). In the dilute limit, $\mathrm{Xs}$ in semiconductors behave as bosons and are expected to undergo $\mathrm{BEC}$ at a temperature several orders of magnitude higher than for atomic $\mathrm{BEC}$ 
because of their light mass. ${ }^{1}$ Following proposals of possible excitonic BEC in 1962 [11,12], theoretical work [13-19] has suggested that Xs could undergo BEC at a critical temperature $\left(T_{c}\right)$ as high as 1 to $10 \mathrm{~K}$, a factor of $10^{6}$ higher than for atoms. The realization of excitonic BEC in semiconductors would open new opportunities in the manipulation of macroscopic quantum coherence because of the greater flexibility and well-developed technology of semiconductor materials [20]. However, excitonic BEC presents both fundamental and practical experimental challenges.

From a fundamental viewpoint, Xs are spatially extended composite particles (Bohr radius: $\mathrm{ax} \approx 10 \mathrm{~nm}-50 \mathrm{~nm}$ ) made of loosely-bound fermions that evolve not in the real vacuum but in an extremely dense $\left(\approx 10^{23}\right.$ particles $\left./ \mathrm{cm}^{3}\right)$ solid matrix. The $\mathrm{X}$ density, $\mathrm{n}_{\mathrm{X}}$, can be widely varied with the photo-excitation intensity, and when $\mathrm{n}_{\mathrm{X}}$ approaches the level where the inter-particle spacing becomes comparable to $\mathrm{ax}_{\mathrm{x}}$, a crossover from Bose to Fermi statistics occurs. Therefore, excitons no longer retain their boson-like character at high densities [21-23]. Moreover, complicated interactions such as screening [24-27] and spin [28-30] effects in the electron-hole (e-h) and exciton systems make prediction of ground state of an high density e-h system challenging. For example, at sufficiently high densities, screening of the e-h coulomb attraction prevents the binding of e-h pairs, and the insulating $\mathrm{X}$ gas can undergo a Mott transition to a conducting e-h plasma (EHP) [31]. In some semiconductors a gas-liquid phase transition can also occur where the $\mathrm{X}$

${ }^{1}$ For an ideal non-interacting three-dimensional bosonic system, the critical temperature of BEC is $T_{c}=\frac{2 \pi \hbar^{2}}{M k_{B}}\left(\frac{n / g}{2.612}\right)^{2 / 3}$, where $\mathrm{n}$ is the density, $\mathrm{g}$ the ground state degeneracy, and $\mathrm{M}$ the mass of bosons. 
gas condenses into an e-h liquid (EHL) [13,14,32,15,33]. Figure 1.1 schematically illustrates possible phases of low to high density e-h systems.

\section{Dilute Exciton Gas Dense Exciton Fluid Electron-Hole Fluid}

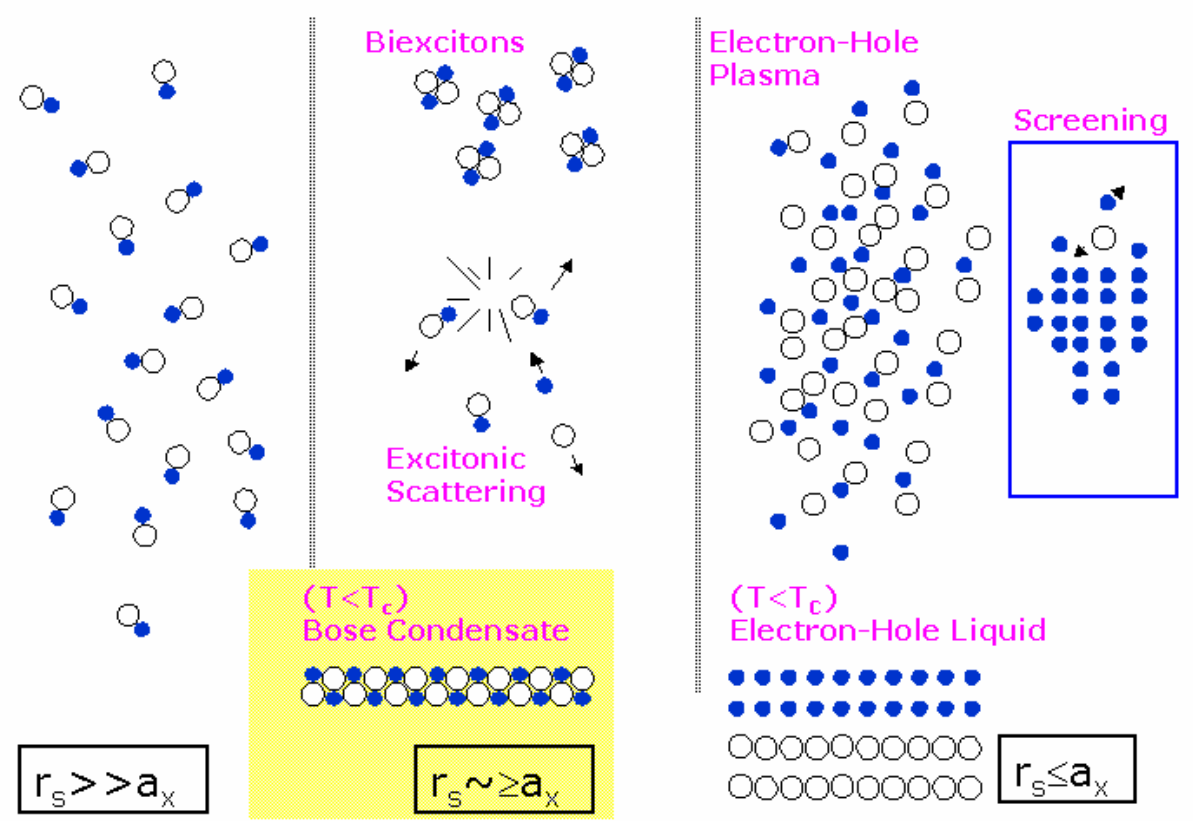

Figure 1.1 Illustration of the electronic and excitonic phases in semiconductors. In the dilute exciton gas, where the mean interparticle distance $r_{s}$ is much larger than the exciton Bohr radius $a_{x}$, the spontaneous annihilation yielding luminescence is the main decay channel. With increasing densities $\left(\mathrm{r}_{\mathrm{s}} \geq \mathrm{a}_{\mathrm{x}}\right)$ excitonic scattering processes and possible biexciton formation mediate the decay process. At high carrier densities $\left(r_{s}\right.$ $\leq a_{x}$ ) the screening of the Coulomb interaction (right inset) leads to the ionization of excitons. The radiative processes are hence dominated by free carrier recombination. It is expected that below a certain critical temperature either the dense exciton fluid or the dense electron-hole fluid can undergo a phase transition, forming an electron-hole liquid (EHL) or a condensed Bose state. The later has not been established experimentally. (Adapted from [34])

Experimentally, though many claims have been made over the past thirty years, the evidence for BEC has not been unambiguous (see for example ref. [35] for reviews).

From a practical viewpoint, the crucial step toward excitonic BEC is the realization of cold statistically degenerate X systems. Xs as quasi-particles of excited semiconductors have a finite lifetime and typically annihilate radiatively on the order of nanosecond in 
direct-bandgap semiconductors. For possible occurrence and observation of excitonic $\mathrm{BEC}, \mathrm{Xs}$ are required at least to cool down via emission of phonons and reach quasiequilibrium in a time much shorter than their lifetime. In semiconductor coupled quantum wells (CQW) under a static electric field perpendicular to the QW-plane (z-direction), the ground state is a spatially indirect exciton (indirect-X) with the electron confined in one QW and the hole in the other [36,37]. These indirect-Xs in CQW possesses several unique properties. (i) The separation of electrons and holes in the z-direction reduces the wave function overlap, resulting in an increased radiative lifetime that in our GaAs/AlGaAs CQW samples is about two orders of magnitude longer than that of the direct-Xs (e-h pairs in the same QW) (see Chapter 4). (ii) Furthermore, the CQW are embedded in a three-dimensional (3D) structure resulting in relaxation of the momentum conservation along the $\mathrm{z}$-direction. Therefore, cooling to the lattice temperature, via emission of bulk longitudinal acoustic (LA) phonons, is about three orders of magnitude faster for Xs in GaAs QWs than that in the bulk [38]. (iii) Finally, the e-h spatial separation give rise to a repulsive dipole-dipole $\mathrm{X}$-X interaction that effectively screens the in-plane disorder potential [39]. Because of these unique features of spatially indirectXs, CQW nanostructures provide new opportunities for discovery of macroscopic collective quantum effects.

The quasi-two-dimensional (quasi-2D) nature of CQW also raises important theoretical issues related to phase transitions in reduced dimensionality. BEC only occurs at $\mathrm{T}=0$ in a homogeneous infinite-plane $2 \mathrm{D}$ system of ideal bosons $[40,41]$, but can occur at finite $\mathrm{T}$ when they are confined in $2 \mathrm{D}$ traps $[42,43]$. Hence producing local confined cold X systems may be a key step to realize excitonic BEC. 
This dissertation describes experimental efforts to characterize properties of spatially indirect-Xs in CQW. The realization, phase diagram, and formation mechanisms of degenerate cold indirect-X systems confined in in-plane localities ('traps', for brevity) are reported $[44,45]$. Contraction of clouds containing more than tens of thousands of excitons within areas as small as $(10 \mu \mathrm{m})^{2}$ below $\sim 10$ Kelvin was observed. Applying the $T_{c}$ formulae for ideal bosons in a square 2D-box [43] to the confined Xs observed gives $\mathrm{T}_{\mathrm{c}} \approx 1 \mathrm{~K}$ for reachable experimental conditions. ${ }^{2}$ Photoluminescence (PL) hundreds microns away from localized excitation spots were also observed in the form of ordered fragmented rings with a extended dark zone in between [46,47]. This signaled macroscopic carrier transport and possible ordering in the system.

The formation mechanisms of these unusual 'naturally' formed cold dense $\mathrm{X}$ systems were not clear. We thoroughly investigated the optical and electrical properties of the CQW sample to provide deeper understanding of the formation mechanisms of these cold exciton systems. These insights in the formation mechanisms offer new strategies for artificially producing cold exciton systems in a more controllable way, where trapping and manipulation of Xs can be engineered. This may lead to opportunities for the realization of $\mathrm{BEC}$ or observation of quantum collective effects in solid-state systems.

${ }^{2}$ For a $2 \mathrm{D}$ box, $T_{c}=\frac{4 \pi \hbar^{2} n}{2 M k_{B} g} \frac{1}{\ln (n S / g)}$, where $\mathrm{n} \approx 5 \times 10^{10} \mathrm{~cm}^{-2}$ is the density, $\mathrm{M} \approx 0.22 \mathrm{~m}_{0}$ the indirect-X effective mass, $S \approx(10 \mu \mathrm{m})^{2}$ the area, and $g=4$ the degeneracy. Here the the confinement across wells is neglected (i.e. assume ideal $2 \mathrm{D}$ excitons). 


\subsection{Outline of thesis}

In the next chapter, we present an introduction to the physics of GaAs structures, Wannier excitons in semiconductors [48,49] and coupled quantum wells [50]. An overview of the photoluminescence and life cycle of excitons is also given. In Chapter 3, we detail both the experimental apparatus and the structures of the CQW samples. Chapter 4 presents an experimental investigation of the properties of the spatially indirect excitons, including the dependence of exciton energy and lifetime on the electric fields and laser excitation intensities. In Chapter 5, we describe the production of statistically degenerate exciton systems confined within in-plane localities and their phase diagram as a function of temperature and excitation intensity. In Chapter 6, we briefly review the ordered necklace-like PL patterns and other photoluminescence centers observed on the same CQW samples. The photocurrent characteristics of the sample and the formation mechanisms of the confined exciton systems in localities are addressed in Chapter 7. In Chapter 8, we review experimental progress and criteria for excitonic BEC. Finally, we summarize the results and discuss future research directions. 


\section{Chapter 2 Background}

\subsection{Introduction}

This chapter provides a general background for understanding the main results of this work. We begin by reviewing GaAs and quantum-well structures, followed by reviews of Wannier excitons and excitons in quantum wells. We will then describe the effects of electric and magnetic fields on the quantum wells, especially the energy levels in coupled quantum wells (CQW). After describing life cycles of excitons, we will end the chapter with a discussion of photoluminescence as a probe of the energy and spatial distributions of optically active excitons.

\subsection{GaAs structures}

Various material parameters and properties of GaAs and related materials are well-known [51]. Here we only review some properties relevant to the thesis. The electronic energy-band structures of bulk GaAs have been extensively studied [5255,51,56,57]. The fundamental absorption edge of GaAs corresponds to direct transitions from the highest valence band to the lowest conduction band at the $\Gamma$ point $(\mathrm{K}=0)$ in the first Brillouin zone (BZ). Near the $\Gamma$ point, the band structure is well described by the effective mass approximation. At $\mathrm{K}=0$ the conduction band electrons exhibit $s$-like symmetry while the valence band electrons exhibit $p$-like symmetry. With the inclusion of spin, there are two s-like conduction bands and six $p$-like valence bands, respectively degenerate at the $\Gamma$ point. The low temperature bandgap is $E_{g}=1.519 \mathrm{eV}$. These bands 
are $\left|S_{1 / 2}\right\rangle$ with total angular momentum $\mathrm{J}=1 / 2,\left|P_{3 / 2}\right\rangle$ with $\mathrm{J}=3 / 2$, and $\left|P_{1 / 2}\right\rangle$ with $\mathrm{J}=1 / 2$ as detailed in ref. [56]. When spin-orbit effects are included, the two split-off hole states $\left(\left|P_{1 / 2}\right\rangle \equiv\left|J=1 / 2, m_{J}= \pm 1 / 2\right\rangle\right)$ drop in energy, opening a gap $\left(\Delta_{0} \approx 0.34 \mathrm{eV}\right.$ at low temperature) between it and the light (lh, $\left.\left|J=3 / 2, m_{J}= \pm 1 / 2\right\rangle\right)$ and heavy-hole (hh, $\left.\left|J=3 / 2, m_{J}= \pm 3 / 2\right\rangle\right)$ bands without affecting the s-like states [58,51]. These bands have, in general, different curvature so that they split away from the $\Gamma$-point $(K \neq 0)$. The electron and hole effective mass can be expressed by $E_{v(c)}(\mathbf{K})=E_{v(c)}(\Gamma)-\frac{\hbar^{2} k^{2}}{2 m_{h(e)}^{*}}$, where $m_{h(e)}^{*}$ can be the electron, heavy-hole, or light hole effective mass. The isotropic effective mass of the electron is $m_{e}^{*}=0.067 m_{0}$, where $m_{0}$ is the electron rest mass. The effective masses of holes in bulk GaAs are also relatively isotropic near the BZ center. The spherically-averaged effective masses of the holes are $m_{h h}^{*}=0.5 m_{0}$ and $m_{l h}^{*}=0.08 m_{0}$ $[51]$.

Due to the enormous advances in the epitaxial crystal growth techniques such as molecular beam epitaxy (MBE) and MOCVD, one can grow alternating layers of different semiconductors with well-controlled thickness (down to one mono-atomic layer precision) and composition. This has provided new systems for both basic physics research and various device applications. The GaAs/AlGaAs heterostructure system is one prime example. Because the lattice parameter difference between $\mathrm{GaAs}$ and $\mathrm{Al}_{\mathrm{x}} \mathrm{Ga}_{1}$ ${ }_{\mathrm{x}}$ As $(0 \leq \mathrm{x} \leq 1)$ is negligible $(<0.15 \%$ at $300 \mathrm{~K})$, we can create lattice-matched heterointerfaces with various band offsets and tailor the barrier heights for particular applications. By sandwiching a layer of GaAs between AlGaAs, a quantum well (QW) is 
created in the growth direction (z-direction). The electrons in the GaAs layer are confined in a one-dimensional (z-direction) potential well whose height is labeled as $\Delta \mathrm{E}_{\mathrm{c}}$. Similarly, the holes are also confined in a potential well with height $\Delta \mathrm{E}_{\mathrm{v}}$. This confinement has a significant effect on the energy states: (i) It lifts the hh-lh degeneracy at the $\Gamma$ point due to the reduction of symmetry. (ii) The energy bands $k_{z}$ has discrete values but are continuous function of $k_{x}$ and $k_{y}$. (iii) The hh and $\mathrm{lh}$ masses are quite anisotropic due to the $p$-like symmetry of the valance bands, though the electron mass is still isotropic. The dispersion equation, taken from the Luttinger Hamiltonian [59], near $\mathrm{K}=0$ gives the functional expression for in-plane (x-y plane, $\|)$ and perpendicular (zdirection,$\perp$ ) hole masses in a [001]-grown QW as:

$$
m_{h h \perp}=\frac{1}{\gamma_{1}-2 \gamma_{2}}, \quad m_{l h \perp}=\frac{1}{\gamma_{1}+\gamma_{2}}
$$

and

$$
m_{h h \|}=\frac{1}{\gamma_{1}+\gamma_{2}}, \quad m_{l h \|}=\frac{1}{\gamma_{1}-\gamma_{2}}
$$

, where $\gamma_{1}=7.10$ and $\gamma_{2}=2.02$ are Luttinger parameters [51].

The masses given by Eq. (2.1) are identical as those for bulk [001] hh and lh masses. In contrast, Eq. (2.2) shows that the in-plane hh (1h) band now has a light (heavy) mass. The masses determined by these formula are $\mathrm{m}_{\mathrm{hh} \|}=0.11 \mathrm{~m}_{0}, \mathrm{~m}_{\mathrm{lh} \|}=0.20, \mathrm{~m}_{\mathrm{hh} \perp}=$ $0.33 \mathrm{~m}_{0}$ and $\mathrm{m}_{\mathrm{l} \perp \perp}=0.09$. These expressions are highly simplified so that they may differ from the real QW case. Nevertheless, they provide indications of the in-plane and perpendicular masses in an approximate manner. More exact calculations of the Luttinger Hamiltonian including the detailed band mixing have been carried out $[60,61]$. The hole 
effective masses in GaAs/AlGaAs heterostructures have been examined by various means such as photoluminescence, magnetooptics, cyclotron resonance, etc. (see for example, references in chapter 9 of [51]). We will report in Sec. 4.6 the measured heavy-hole exciton dispersion and effective mass $\left(\approx \mathrm{m}_{\mathrm{e}}+\mathrm{m}_{\left.\mathrm{hh}_{\mid}\right)}\right)$utilizing magnetooptics.

\subsection{Wannier excitons}

In semiconductors, the excited electron and hole interact attractively according to the Coulomb law modified by the medium, $-\mathrm{e}^{2} / \varepsilon \mathrm{r}_{\text {eh }}$. Here $\varepsilon$ is the dielectric constant and $\mathrm{r}_{\mathrm{eh}}$ is the distance between the excited electron and hole. Due to the relatively large dielectric constants in semiconductors, the excited electrons and holes are loosely coupled with most of their free particle features retained. Therefore, the wavefunction of these composite bound electron-hole pairs, known as Wannier excitons [48,62,49,63 ], includes a slowly varying envelope with their unmodified Bloch functions.

\subsection{Excitons in quantum wells}

Bound and continuum states of excitons in $3 \mathrm{D}$ are basically similar to those found in the hydrogen atom discussed in general quantum mechanics books. The energies of the bound states are given by

$$
E_{n}^{(3 D)}=E_{g}-\frac{R y^{*}}{n^{2}}, \quad R y^{*}=\frac{\mu}{2}\left(\frac{e^{2}}{4 \pi \varepsilon \hbar}\right)^{2},
$$

where $\mathrm{Ry}^{*}$ is the Rydberg energy scaled by the dielectric constant and the reduced mass $\left(\mu^{-1}=\mathrm{m}_{\mathrm{e}}^{-1}+\mathrm{m}_{\mathrm{h}}^{-1}\right)$ of the exciton. The theory of excitons is similar in strictly twodimensional (2D) limit, where the thickness in the z-direction is assumed to be zero. Analytical solutions for the 2D exciton problems in momentum-space or real-space 
(coordinate-space) have been solved or discussed, for example, in refs. [64,57,65]. In this limit 2D case, the energy levels are given by

$$
E_{n}^{(2 D)}=E_{g}-\frac{R y^{*}}{(n-1 / 2)^{2}} .
$$

Therefore, the binding energy of excitons in $2 \mathrm{D}$ is four times that in the 3D. For finite thickness quantum well structures, theory of excitons can be found for example in refs. [66-71].

\subsubsection{Electric and magnetic fields}

Electric and magnetic fields are valuable probes of electronic systems. We discuss the effects of electric and magnetic fields on quantum wells structures separately in the sections below.

\subsubsection{Quantum wells under electric field}

An electric field is commonly used to drive a current through a conductor; however, useful information or applications can be extracted by applying an electric field to an insulator as well. In the bulk case, a strong electric field causes a change in optical absorption near the band edge - known as the Franz-Keldysh effect. This is even more powerful when the electrons and holes are confined in a QW. An electric field in excess of $10^{5} \mathrm{kV} / \mathrm{cm}$ can be applied in the growth direction of the QW structures either via a Schottky barrier or by inserting the structure into the intrinsic part of a p-i-n or n-i-n junction. The consequent dramatic effects on the optical properties of quantum well structures have been extensively studied from both fundamental and practical point of view [72-76]. With a perpendicular electric field on the QW, significant changes in the optical absorption, reflection, and PL spectra can be observed and are attributed to modifications of the spatial confinement of the electron and hole wave functions. The 
shift of the absorption edge caused by the so called quantum confined Stark effect (QCSE) has found applications in high speed optical modulators [77].

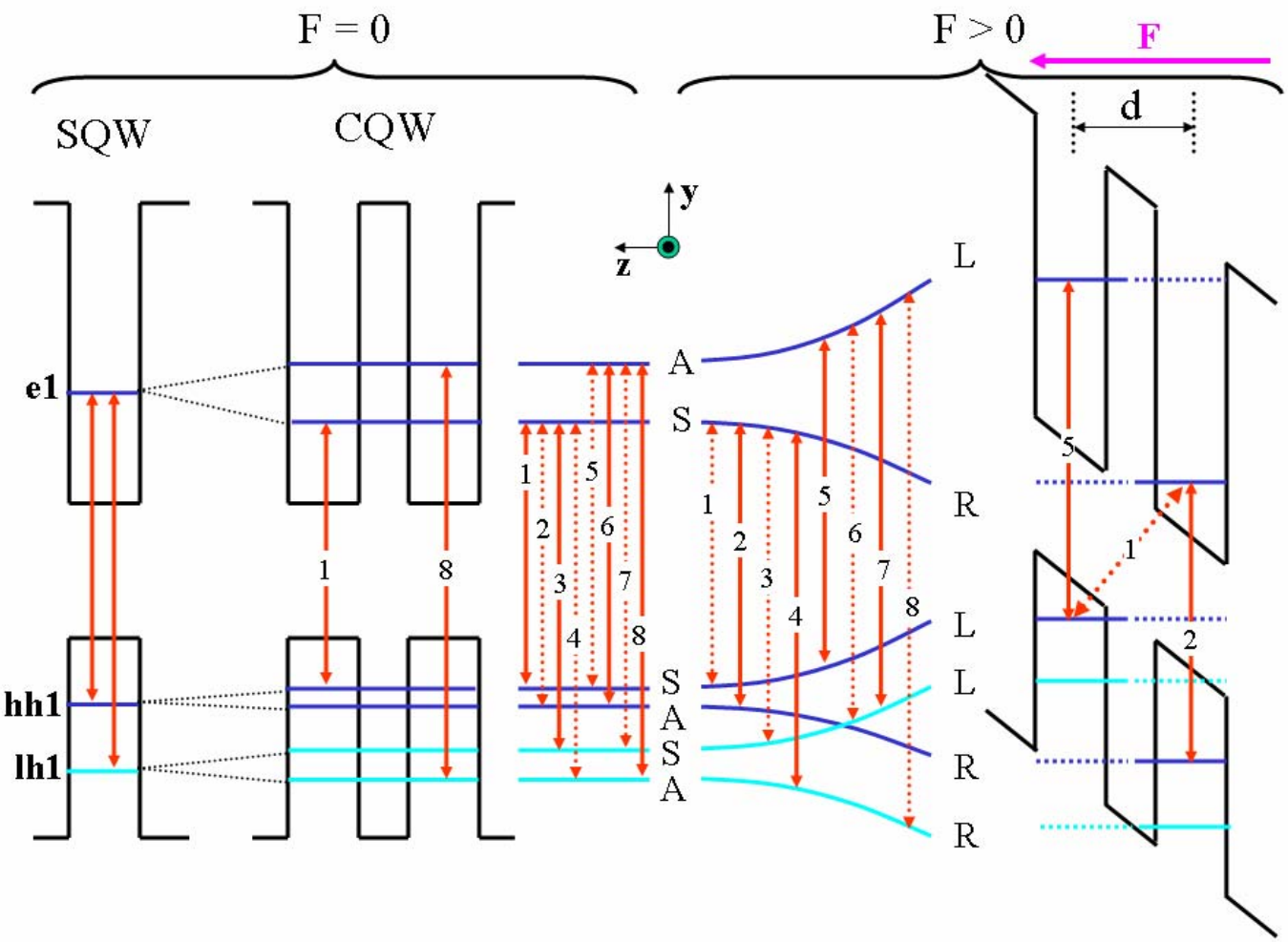

Figure 2.1 Schematic diagram of energy levels and transitions in a CQW under flat-band (electric field $\mathrm{F}=0$ ) and electric field $(\mathrm{F}>0)$ conditions. Only the first electron, heavy-hole, and light hole subbands are shown and the energy levels are not drawn to scale. Symbols: S - symmetric states; A - asymmetric states; L - states with probability densites peaked in the left QW; R - states with probability densites peaked in the right QW (Adapted from [50])

Pairs of QWs structures with an inter-well barrier sufficiently narrow that the electron and/or hole wave functions in the two wells overlap considerably were also employed to study the electric field effects [78-82]. We will refer to such structures as coupled double quantum wells $(\mathrm{CDQW})$ or coupled quantum wells $(\mathrm{CQW})$ for brevity. In an unperturbed system of two identical QWs separated by a thin barrier, degenerate single QW states split into symmetric (S) and antisymmetric (A) doublet states (Figure 2.1). In an isolated single $\mathrm{QW}$, the effect of the electric field is to reduce both conduction 
and valence band energies and exciton binding energies. In a CQW, the split levels of each of the coupled electronic states move in opposite direction as a function of the applied electric field (Figure 2.1). Transitions between these levels are essentially either: (a) spatially indirect (inter-well), associated with recombination of electrons in one well and holes in the other, or (b) spatially direct (intra-well). In the case of spatially indirect transitions, the exciton radiative lifetime is increased due to the reduction in spatial overlap between the electron and hole wave functions.

Figure 2.1 shows the feature energy states, positive and negative Stark shifts, and the crossings of some levels [50]. Observable optical transitions are determined by parity symmetry between the states for symmetric CQW. In the absence of an electric field (the flat-band condition), the coupled states possess definite parity under reflection in the zdirection. In this condition, the only dipole allowed transitions are between electron and hole states having the same z-directed symmetry. When a perpendicular electric field is applied to the CQW, the wave functions are distorted, reflection symmetry destroyed, selection rules are relaxed and all transitions become dipole allowed. As shown in Figure 2.1, the symmetry allowed transitions under the flat-band condition become inter-well (spatially indirect) transitions under an applied field, whereas the previous symmetryforbidden transitions become intra-well (direct) transitions. Note that transition 1, which is the main transition observed in PL and symmetry allowed in the absence of a bias voltage, becomes a spatially indirect (inter-well) transition between electrons and holes in separate wells with applied electric fields. The lowest exciton state formed by this transition [electron (e1) to heavy-hole (hh1)] is the most interesting state for investigation of exciton condensation. It was investigated through $\mathrm{CW}$ PL or time-resolved PL 
extensively in this dissertation. We refer to the inter-well excitons as spatially indirect excitons (indirect-Xs) and intra-well excitons as direct excitons. The plethora of different transitions involved can be effectively explored with PL excitation (PLE) and photocurrent spectroscopy. In this dissertation, only a limited number of such measurements are addressed.

\subsubsection{Quantum wells under magnetic field}

The quantum-mechanical effects of an electric field are usually not too divergent from that expected classically. A magnetic field has more profound effects, particularly on low dimensional systems. The continuous density of states of a two-dimensional electron gas splits into a discrete set of $\delta$-function Landau levels $[83,65]$, which manifest in the longitudinal conductivity as the Shubnikov-de Hass effect [84]. In a twodimensional electron gas, the integer quantum Hall effects and fractional quantum Hall effects are well-known phenomena [85-87]. These effects and general magneto-optical properties of excitons or electron/hole gases in quantum wells are beyond the scope of this dissertation. One aspect of interest is the magnetic enhancement of the effective mass of the spatially indirect excitons in CQW. This is described in Sec. 4.6

\subsection{Photoluminescence and life cycle of excitons}

After the creation of electron-hole (e-h) pairs by optical or other excessive energy excitation, thermal equilibrium is restored through the carrier relaxation, exciton formation, and eventual recombination giving rise to observable luminescence (Figure 2.2). The radiative recombination processes appear to dominate the non-radiative processes in QWs at low temperatures. However, electron-hole pairs in QWs do relax to respective sub-bands via emission of longitudinal acoustic (LA) phonons or optical phonons (LO, for $\mathrm{T}>100 \mathrm{~K}$ ), or through carrier-carrier scattering [88]. In CQW with 
structural and/or electric field induced asymmetry, the probability densities for the ground and first excited states are peaked in separate wells. Impurity or optical phonon assisted tunneling and scattering through the coupling barrier allows carriers created in the anti-symmetric excited states to relax quickly to the symmetric ground state. Electron tunneling time of tens of picoseconds for electric fields of a few tens of $\mathrm{kV} / \mathrm{cm}$ was reported for CQW with AlGaAs barrier thickness $\sim 40-50 \mathrm{~A}^{\circ}[89,90]$. Relaxation of electrons from the anti-symmetric to symmetric sub-band states through LO phonon scattering is possible when the sub-band transition energy exceeds the LO photon energy $\left(\hbar \omega_{\mathrm{LO}}=36 \mathrm{meV}\right.$ in GaAs [51]). LO phonon scattering time under $10 \mathrm{ps}$ has been predicted in CQW for experimental conditions similar to those investigated here [90]. Impurity assisted tunneling between the excited and ground state sub-bands for heavyholes within symmetric $\mathrm{GaAs} / \mathrm{Al}_{0.3} \mathrm{Ga}_{0.7} \mathrm{As} \mathrm{CQW}$ similar to those studied were reported, which showed a $340 \mathrm{ps}$ tunneling time for electric field of $\sim 16 \mathrm{kV} / \mathrm{cm}$ [91].

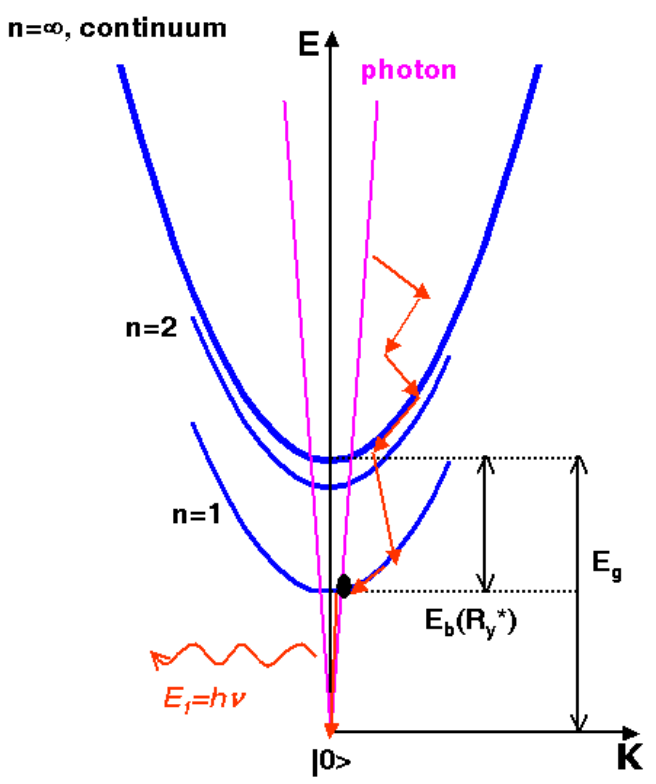

Figure 2.2 Energy-momentum dispersion and an example of the life cycle of excitons. The red arrows represent example relaxation processes after formation of an exciton. 
Upon relaxation to the respective ground sub-band states in times on the order of sub-nanoseconds, the mutually coulomb attraction of electrons and holes dominates to form excitons (Xs). These initial Xs are energetic (hot) with finite center-of-mass (CM) momenta $(\mathbf{P}=\hbar \mathbf{K} \neq 0)$. After relaxation (cooling) through $\mathrm{X}-\mathrm{X}, \mathrm{X}$-free carrier, and $\mathrm{X}$ acoustic phonon scattering $[88,92]$ to reach a quasi-thermal equilibrium, cold Xs with $\mathrm{K} \approx 0$ eventually annihilate and emit detectable photons (photoluminescence). At low temperatures X-LA phonon scattering dominates the cooling process, exhibiting a characteristic sub-nanosecond relaxation time [38]. Note that the relaxation time can increase depending on the interface roughness and localization.

The CQW are embedded in a three-dimensional structure resulting in relaxation of the momentum conservation along the QW growth direction (z-direction). Therefore, cooling to the lattice temperature, via emission of bulk longitudinal acoustic phonons, is about three orders of magnitude faster for Xs in GaAs QWs than that in the bulk [38]. This cooling is an elastic process that conserves both in-plane momentum and the total energy. The energy of a phonon mode with momentum $\hbar \mathbf{K}_{\|}$is given by $\hbar \mathbf{K}_{\|} v_{s}$, where $v_{s}$ is the sound velocity. A free $\mathrm{X}$ with mass $\mathrm{M}$ and wave vector $\mathrm{K}$ cannot be scattered into a lower kinetic energy state by emission of a single acoustic phonon if the slope of the $\mathrm{X}$ energy dispersion at $\mathrm{K}$ is less than $\hbar v_{s}$, i.e. if $\hbar K<M v_{s}$. A bottleneck for cooling by acoustic phonon scattering occurs at a free $\mathrm{X}$ energy of $\frac{1}{2} M v_{s}$. For bulk acoustic phonons in GaAs, $\mathrm{v}_{\mathrm{s}} \approx 3.4 \times 10^{7} \mathrm{~cm} / \mathrm{s}$. For heavy-hole spatially indirect-Xs in CQW, $\mathrm{M} \approx 0.22 \mathrm{~m}_{0}$ (see Sec. 4.6 for measured effective mass), where $\mathrm{m}_{0}$ is the free electron rest mass. The low temperature limit for acoustic phonon assisted cooling is then given by $\frac{1}{2} M v_{s} / k_{B} \sim 0.1 \mathrm{~K}$. For a lattice temperature much higher than this values, as in most 
cases of PL experiments herein, thermalization of free Xs can proceed without encountering this bottleneck.

Under a static electric field perpendicular to the QW-plane (z-direction), the ground state is a spatially indirect- $\mathrm{X}$ with the probability amplitudes of the electron and hole wave functions peaked in separate adjacent QWs. This separation reduces the electron and hole wave function overlap, resulting in an increased radiative recombination lifetime that in our GaAs/AlGaAs CQW samples can be $\approx 100$ ns, about two orders of magnitude longer (sec. 4.4) than that of the direct-X (e-h pairs in the same QW). The long indirect-X lifetime leads to a high density quasi-thermal X distribution, in which collective quantum effects may be observed [93-98].

\section{Lifetime}

QW excitons interact with a quasi-continuum of photon states, which give rise to a finite lifetime. Assuming a thermal distribution of the in-plane center of mass energy $\hbar^{2} K^{2} / 2 M$, and using the fact that because only excitons with in-plane wave vector $\mathrm{K}$ equal to the photon wave vector $\hbar \omega n / c$ can radiate, the Fermi Golden Rule gives a radiative lifetime of free excitons proportional to the temperature $T$. On the other hand, the radiative lifetime decreases with decreasing well thickness due to a better overlap of the envelope electron- and hole- wave functions [99]. The picture of free-exciton radiative decay is not valid when excitons are localized and when the characteristic coherence length of the exciton $l_{c}$ satisfies the condition that $\hbar^{2} / 2 M l_{c}^{2}>k_{B} T$. The localized exciton lifetime becomes temperature independent and is generally in the 100 ps range. In moderate quality QW samples, the recombination time can be governed by this and other non-radiative processes, which gives rise to a recombination lifetime that 
decreases with temperature. Finally, at room temperature most excitons are thermally ionzed and the radiative recombination process is essentially governed by band-to-band bimolecular processes.

\section{PL: k-selection rule}

Photoluminescence results from the radiative recombination of optically active, bound or unbound, electron-hole pairs with $\mathrm{CM}$ momentum $\mathrm{P} \approx 0[100,99]$. Only $\mathrm{X}$ states which can decay into photons with conservation of both momentum and energy can contribute to the PL signal. The momentum of PL photons emitted has a z-component generated from the Fourier components of the quantum confined electron and hole wave

functions; whereas the in-plane momentum component is determined by the X center-ofmass momentum. For free excitons with homogeneous linewidth $\hbar \Gamma$, then all states satisfying the following relationship can becom optically active:

$$
\frac{\hbar^{2} K_{\text {o.a. }}^{2}}{2 M} \leq \frac{\hbar \Gamma}{2}
$$

For radiative recombination lifetimes on the order of $100 \mathrm{~ns}$ in a two level system, the homogeneous linewidth is on the order of $10^{-8} \mathrm{eV}$. This is much less than the thermal energy of Xs, which is on the order of the spread in kinetic energy $k_{B} T \sim \frac{\hbar^{2} \Delta K^{2}}{2 M}$. Therefore, optically active Xs constitute only a small fraction of a thermally quasiequilibrium distribution of exciton population.

\section{PL Linewidth}

Molecular beam epitaxy grown QW structures have typical thickness fluctuations on the order of one monolayer or greater. The resultant confining potential variation in the QW contributes to an inhomogeneous broadening of exciton linewidth. Hence, the X 
PL linewidth is better described by the convolution of the homogeneous and inhomogeneous linewidths with the latter being the dominant term [101].

Inelastic electron-phonon scattering processes and other scattering processes due to coulombic impurities, alloy fluctuations etc. all contribute to the homogeneous linewidth of X transition in QWs. The homogeneous linewidth of excitonic lines in GaAs has been found to be well approximated by the following equation:

$$
\Gamma=\Gamma_{0}+\gamma T+\Gamma_{L O} \frac{1}{e^{\hbar \omega_{L O} / k_{B} T}-1}
$$

with $\Gamma_{0}=0.5-1 \mathrm{meV}$ represents the temperature-independent contribution from defect scattering, $\gamma=5-10 \mu \mathrm{eV} / \mathrm{K}$ is due to acoustical phonons scattering, while $\Gamma_{\mathrm{LO}}=5 \mathrm{meV}$ is due to longitudinal optical phonons ( $\left.\hbar \omega_{L O}=36 \mathrm{meV}\right)$ scattering [102].

At low temperature, the excitonic linewidth is inhomogeneously broadened principally because of interface roughness.

\section{PL intensity}

As a result of the disorder present in QWs, a finite fraction of the Xs can be trapped at potential minima depending on the amount of disorder and the lattice temperature. At low lattice temperature, more Xs are trapped by disorder due to the reduced thermal activation energy available to detrap them. These Xs with localized wave functions are delocalized in momentum space, which leads to enhanced radiative recombination. Therefore, the absolute PL intensity increases with decreasing temperature as more localized Xs become optically active while at the same time more excitons are localized at traps. 
The PL spectrum is a measure of the rate of photons emitted at a particular energy $\left[\operatorname{PL}_{\mathrm{I}}(\varepsilon)\right]$, which depends inversely upon the radiative recombination lifetime $[\tau(\varepsilon)]$, and directly on the density of available $\mathrm{X}$ states $[\mathrm{DOS}(\varepsilon)]$ as well as the occupancy of the $\mathrm{X}$ state $[\mathrm{f}(\varepsilon)]$ for a given $\mathrm{X}$ energy:

$$
P L_{I}(\varepsilon) \propto \frac{1}{\tau(\varepsilon)} \operatorname{DOS}(\varepsilon) f(\varepsilon) .
$$

In a disordered 2D potential, the density of states can be described by a Voigt distribution reflecting the intrinsic Lorentzian line-shape of the free Xs convolved with a broader Gaussian due to inhomogeneous broadening [103]. The density of states is independent of the temperature or X density. Changes in the PL line shape are thus determined by the occupancy of available exciton states and the kinetic processes by which vacated exciton states are subsequently repopulated.

\section{Polariton}

Finally, for the experimental configuration shown in Figure 3.4, the incident laser excitation is almost normal to the QW plane and has a polarization parallel to the plane. As a result, due to conservation of momentum, quantum well exciton polariton modes are not directly excited [104,105]. In addition, although the spontaneously emitted PL propagates generally in all directions with random polarizations, the finite numerical aperture of the optical system only extracted PL emissions with an angle less than $\sim 4^{\circ}$ relative to the normal z-direction within the semiconductor. Therefore, the polariton modes propagating in the QW plane were not coupled into the photoluminescence extraction cone and thus were not detected. 


\subsection{Summary}

In this chapter, we reviewed some basic properties of GaAs, the electronic states of GaAs CQW samples and the effects of an applied electric field. We outlined some of the properties of excitons in semiconductor nanostructures. We also reviewed exciton photoluminescence which is an important tool for probing the spatial and energy distribution of optically active excitons. 


\section{Chapter 3 Experimental Setup}

\subsection{Introduction}

This chapter provides a description of the photoluminescence (PL) experiments studied with coupled quantum wells (CQW) in this work. We will begin with a description of the CQW sample used in the experiments, including the sample structure and processing procedure. Next we will describe the experimental apparatus and setup, including the cryogenic systems, laser systems and the microscopic PL ( $\mu-\mathrm{PL})$ imaging and spectroscopic setups. We will then describe the time-resolved PL measurements with a time-correlated single photon counting system. We conclude with a description on photocurrent measurements.

\subsection{Sample structure and preparation}

The GaAs/AlGaAs coupled quantum well (CQW) sample studied consisted of two $80 \AA$ GaAs wells separated by a $40 \AA \mathrm{Al}_{0.33} \mathrm{Ga}_{0.67} \mathrm{As}$ barrier with an overall $n^{+}-i-n^{+}$ structure. The sample was grown by Ken L. Campman in Professor Art Gossard's group at UC Santa Barbara. Table 3.1 details the layer structure of the CQW sample as grown by the molecular beam epitaxy (MBE).

Mesas (ranging from $\sim 400$ to $1000 \mu \mathrm{m}$ squares on a single chip) were defined by standard photolithography and wet chemical etching. $\mathrm{Au} / \mathrm{Pd} / \mathrm{Ge}$ alloy was deposited by e- 
beam evaporation to form ohmic contacts with square openings (ranging from $\sim 300$ to $800 \mu \mathrm{m}$ squares) for the photoexcitation and extraction of photoluminescence. ${ }^{3}$

Table 3.1 Composition details for the CQW sample.

Top Surface

\begin{tabular}{lll}
\hline Top electrode and cap layer & $\mathrm{GaAs}\left(\mathrm{n}+-5 \times 10^{17} \mathrm{~cm}-3\right)$ & $1050 \AA$ \\
\hline Top spacer layer & $\mathrm{Al}_{\mathrm{x}} \mathrm{Ga}_{1-\mathrm{x}} \mathrm{As}(\mathrm{x}=0.33$, intrinsic) & $2000 \AA$ \\
\hline Quantum well & $\mathrm{GaAs}_{\text {intrinsic })}$ & $80 \AA$ \\
\hline Barrier layer & $\mathrm{Al}_{\mathrm{x}} \mathrm{Ga}_{1-\mathrm{x}} \mathrm{As}(\mathrm{x}=0.33$, intrinsic) & $40 \AA$ \\
\hline Quantum well & $\mathrm{GaAs}_{\text {intrinsic })}$ & $80 \AA$ \\
\hline Bottom spacer layer & $\mathrm{Al}_{\mathrm{x}} \mathrm{Ga} a_{1-\mathrm{x}} \mathrm{As}(\mathrm{x}=0.33$, intrinsic) & $2000 \AA$ \\
\hline Bottom electrode layer & $\mathrm{GaAs}\left(\mathrm{n}+-5 \times 10^{17} \mathrm{~cm}-3\right)$ & $3050 \AA$ \\
\hline Superlattice & $\mathrm{AlAs} / \mathrm{GaAs}$ end with $\mathrm{AlAs}$ & $20 \AA / 20 \AA \times 5=200 \AA$ \\
\hline Buffer layer & $\mathrm{GaAs}$ (intrinsic) & $8000 \AA$ \\
\hline Substrate & $\mathrm{GaAs}(\mathrm{n})$ & Crystal orientation $[100]$ \\
\hline
\end{tabular}

Figure 3.1 shows a processed mesa and a photo of a processed chip mounted on a die. Forward (positive) bias is defined as electron flow from the bottom contact through the wells and barriers to the top contact. (i.e., The bottom contact is grounded to the case.)

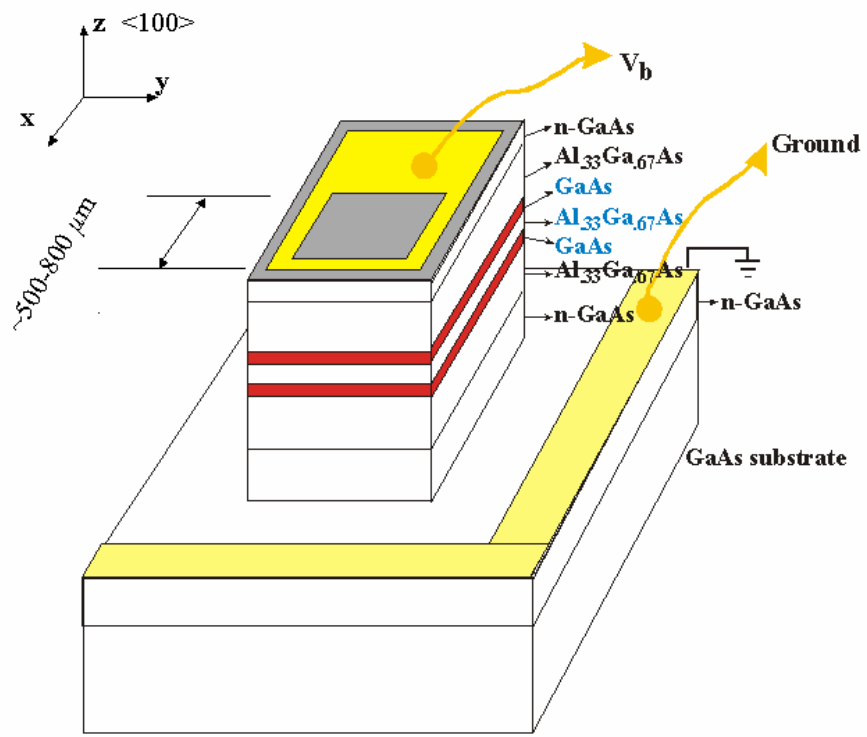

Figure 3.1 A cartoon of a processed mesa.

${ }^{3}$ See Appendix for detailed sample processing procedures. 


\subsection{Experimental apparatus and setup}

\subsubsection{Cryogenic systems}

The coupled quantum well devices were maintained at a constant bath temperature by adjusting the heating power and the flow of helium vapor. The mounted devices were inserted into a socket embedded within a copper block and placed within the optically and electrically accessible sample chamber of (i) a Janis Super VariTemp cryostat, similar to the one shown in Figure 3.2, or (ii) an Oxford 12 Tesla magnet cryostat.

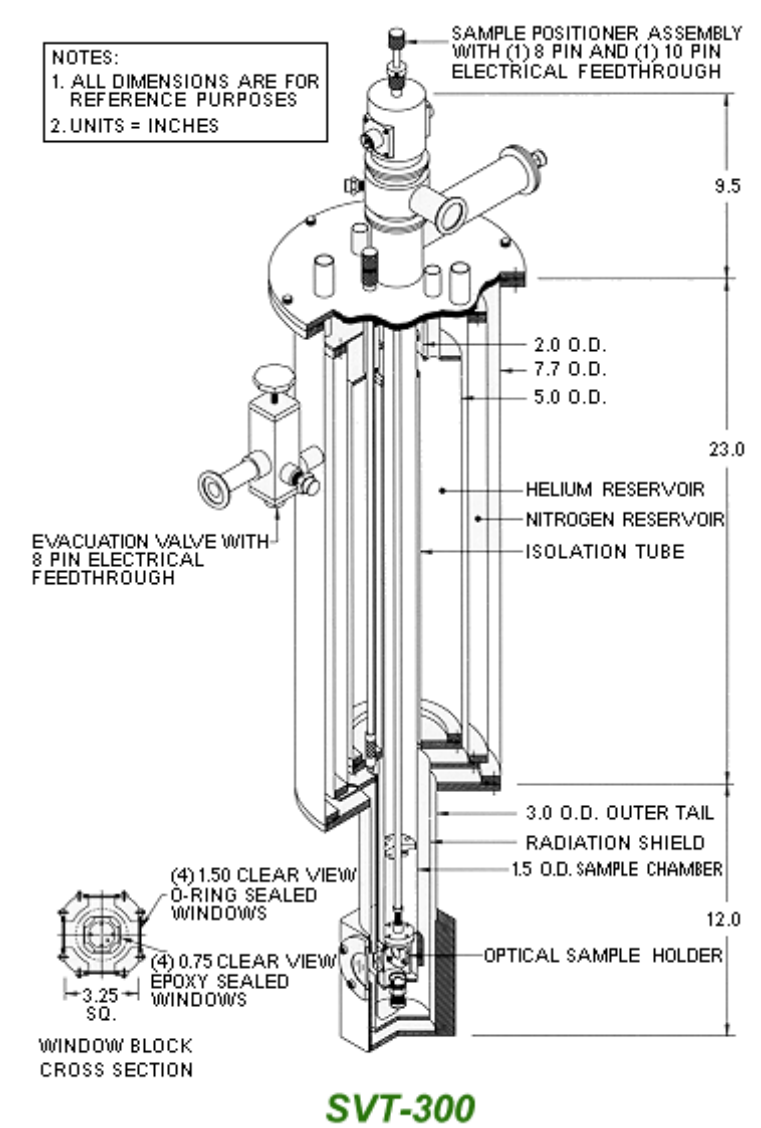

Figure 3.2 Schematic of a Janis Super VariTemp (SVT) cryostat with optical access windows used for the low temperature photoluminescence experiments (Adapted from Janis, http://www.janis.com).

Some of the data were measured inside a magneto-optic cryostat designed and built by Oxford Instruments, Inc. The magnet itself was a superconducting split-coil 
magnet which operates between 0 and 12 Tesla, and is uniform to better than $0.25 \%$ within a $10 \mathrm{~mm}$ diameter volume in the center of the coils. A microscope objective ${ }^{4}$ was installed inside the cryostat near the center of the split coil magnet. The focus of the objective was adjusted at room temperature before cooling down the magnet cryostat. A variable temperature sample cell created a sample space in the uniform area of the magnetic field, with optical access both along the magnet axis and perpendicular to the field direction.

The samples were fixed to a die mounted on a flat metal sample holder. Once thermal equilibrium was reached, the sample position was stable to within $\approx 2 \mu \mathrm{m}$. A capillary tube connected the sample space to a helium reservoir. By opening a needle valve in the capillary tube and regulating the pumping rate on the sample space, it was possible to fill the bottom of the sample cell with superfluid He-4 at a temperature of $\approx 1.7 \mathrm{~K}$. In the Janis cryostat, the sample temperature was measured with a Lakeshore Cryogenics Si diode sensor attached near the sample and a controller/monitor. In the Oxford magnet cryostat, the temperature was determined by measuring the resistance of an ITS-90 calibrated Cernox resistor under a four-lead scheme with a lock-in amplifier.

A heater was attached to the sample holder above the sample mounted. By regulating the He-4 gas flow and voltage applied to the heater, a stable sample temperature between $1.7 \mathrm{~K}$ and $\sim 30 \mathrm{~K}$ was reached.

\footnotetext{
${ }^{4}$ Mututoyo infinitely corrected objective - M Plan Apo 10x: numerical aperture N.A. $=0.28, \mathrm{f}=20 \mathrm{~mm}$, and working distance $=33.5 \mathrm{~mm}$. This objective consists of no compound lenses and can sustain at cryogenic temperatures if care has been taken to avoid thermal shock.
} 


\subsubsection{Laser systems and excitation sources}

Photoluminescence was excited by a HeNe laser or index guided AlGaInP laser diodes (LDs) with spectral profiles given in Figure 3.3. LDs were mounted onto an adjustable focal length collimating optics with a heat sink and driven by (i) a current source for the $\mathrm{CW}$ mode or (ii) a fast pulse generator ${ }^{5}$ for the pulsed mode. A gradient neutral density filter ${ }^{6}$ combined with other neutral density filters were inserted in the optical path to vary the excitation intensity over more than three orders of magnitude. The laser beam was either (i) defocused by a combination of a lens and rotary holographic diffuser to a $\sim 500-700 \mu \mathrm{m}$ spot diameter for uniform excitation or, (ii) focused down to a minimum of $\sim 10 \mu \mathrm{m}$ diameter spot for localized excitation. For the uniform excitation, a rotary diffuser was added in the optical path to eliminate speckle patterns. Experiments with varying excitation energy were also performed. The excitation source was the white light from an ORIEL tungsten lamp dispersed by a SPEX 270M monochromator equipped with a $300 \mathrm{gr} / \mathrm{mm}$ grating blazed at $400 \mathrm{~nm}$. This provided a $\sim 10 \mathrm{~nm}$ bandwidth light from $\sim 400 \mathrm{~nm}$ to $800 \mathrm{~nm}$ with incident power $20 \mu \mathrm{W}$ or more on the sample surface.

The laser power was measured by a Newport optical power meter ${ }^{7}$ placed before the cryostat windows. The laser power $\left(P_{\text {laser }}\right)$ specified in the dissertation was the power deposited on the CQW sample surface, taking into account the transmission loss in transversing the cryostat windows.

\footnotetext{
${ }^{5}$ AVTech AVPP-1-B-P-PN-OT 1MHz pulse generator with 100ps rise and fall time \& pulse widths up to 100ns.

${ }^{6}$ Newport 925B Compensated Attenuator include a matched pair of linear variable metallic neutral density filters, providing high resolution intensity control without introducing a gradient in the intensity distribution.

${ }^{7}$ Newport Optical Power Meter Model 1830-C equipped with a Model 818-ST detector head.
} 


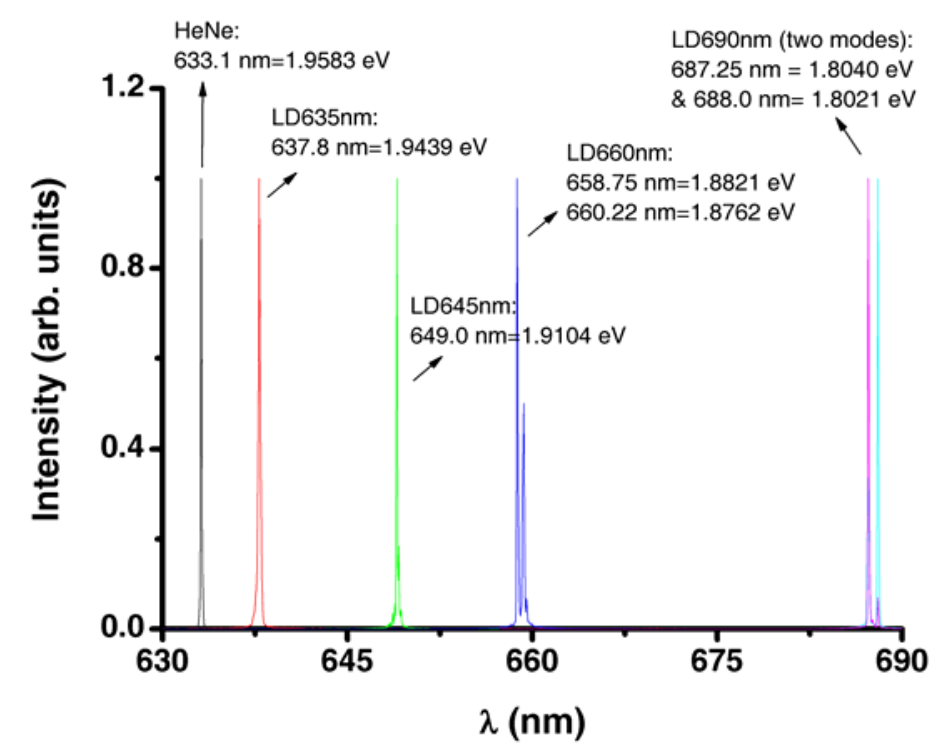

Figure 3.3 Spectral profile of the HeNe laser and laser diodes used as the excitation sources.

\subsection{Optical properties: Photoluminescence}

The photoluminescence (PL) excitation frequency (energy) and intensity used to characterize material parameters are chosen to probe different regions and energy states in the sample. Because PL spectroscopy is a highly selective and extremely sensitive probe of discrete electronic states, features of the emission spectrum such as the signal intensity peak energy $\left(\mathrm{E}_{0}\right)$, and linewidth $(\Gamma)$ are commonly used to identify surface, interface, and impurity states, to gauge alloy disorder and interface roughness, and to determine the quality of the sample. Variation of the PL intensity with an applied bias can be used to map the electric field of a sample. For pulsed excitation, the transient PL intensity provides information on the decay process (e.g. lifetime) of non-equilibrium states. In addition, thermally activated processes cause changes in PL intensity, linewidth, and lifetime with temperature, providing further information regarding the interactions among excited carriers of the probed state. PL analysis is nondestructive and timeresolved PL can be very fast, making it useful for characterizing the most rapid processes 
in a material. However, PL analysis is only sensitive to optically active states. Other techniques are required to study states that couple weakly with light.

The experimental arrangement shown schematically in Figure 3.4 consists of both a spatially- and temporally-resolved microscopic PL ( $\mu$-PL) to study these various aspects of PL. Figure 3.4 is a schematic of the $\mu$-PL imaging and spectroscopy setup. The system has a diffraction limited spatial resolution of $\sim 1.7 \mu \mathrm{m}$ and time resolution of $\sim 40$ ps. The time-integrated PL tomography (PL imaging), spatially-resolved PL ( $\mu-\mathrm{PL})$ spectroscopy, and time-resolved PL setups are discussed separately in the following two sections.

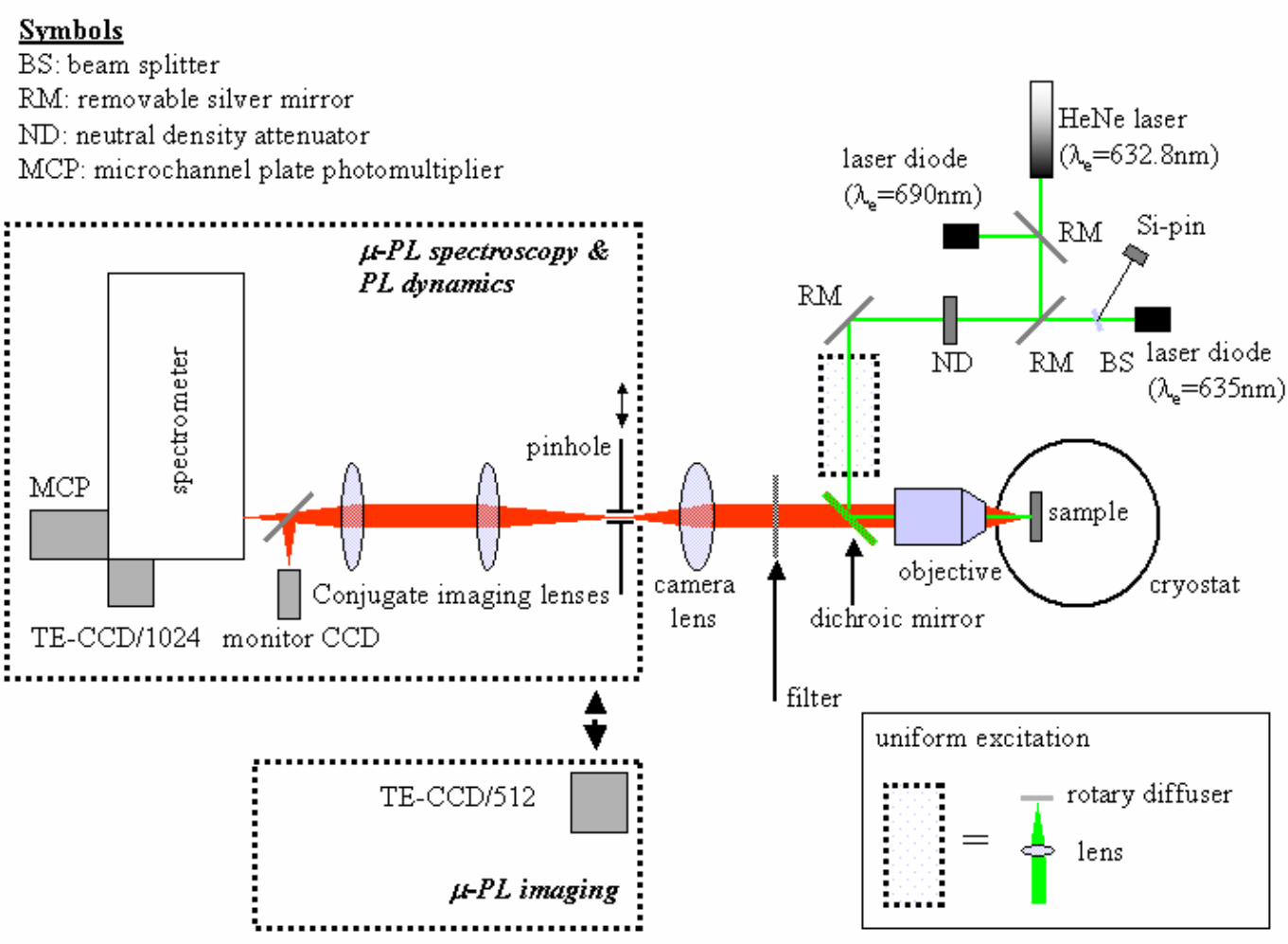

Figure 3.4 Schematics of the PL tomography (PL imaging), spatiallyresolved ( $\mu-\mathrm{PL})$ spectroscopy, and time-resolved PL (PL dynamics) setups. 


\subsubsection{Photoluminescence tomography and $\mu$-PL spectroscopy}

The sample was excited through a long working distance objective ${ }^{8}$ by the laser or incoherent light source described in previous section. The luminescence is collected by the same focusing objective, i.e., confocal configuration, and imaged through a camera lens $(f=300 \mathrm{~mm}$ ), giving a magnification of $\sim \times 15$. Two series of experiments were performed: (i) photoluminescence (PL) imaging by a TE-cooled CCD camera ${ }^{9}$ with a 10 $\mathrm{nm}(\sim 21 \mathrm{meV})$ bandpass interference filter centered at the indirect-X emission line (Figure 3.5) for which a $\sim 2 \mu \mathrm{m}$ spatial resolution is achieved; and (ii) diffraction limited $(\sim 1.7 \mu \mathrm{m})$ spatially resolved PL spectra obtained by scanning a pinhole in the confocal image plane. The PL-imaging (PL tomography) provides direct information on the spatial distribution of the Xs within the filter bandwidth, whereas the PL-scans ( $\mu$-PL) determined the local $(\sim 1.7 \mu \mathrm{m})$ energy distribution of the optically active indirect-Xs with a $\sim 0.1 \mathrm{meV}$ spectral resolution.

In case (ii), spatially resolved spectra were measured with a confocal microscope scheme. Pinholes with different size (typically $\phi=50 \mu \mathrm{m}$ in the conforcal image plane, equivalent to $\phi \approx 3 \mu \mathrm{m}$ on the sample) were installed in the confocal image plane. By scanning the pinhole in the confocal image plane, one can detect local PL in a welldefined way. The transmitted PL emissions were then spectrally resolved by a spectrometer ${ }^{10}$ and captured by a CCD camera ${ }^{11}$. The spectra were digitalized by a

\footnotetext{
${ }^{8}$ Mututoyo infinitely corrected objective - M Plan NIR 10×: numerical aperture N.A. $=0.26, \mathrm{f}=20 \mathrm{~mm}$, and working distance $=30.5 \mathrm{~mm}$.

${ }^{9}$ Princeton Instrument TEA/CCD-512TKB: $512 \times 512 ; 13.8 \times 13.8 \mathrm{~mm}$ overall; $27 \times 27 \mu \mathrm{m}$ and quantum efficiency $=65 \%$ for $\lambda=800 \mathrm{~nm}$.

${ }^{10}$ Acton Spectra Pro750: f=750mm; Grating: $600-\mathrm{g} / \mathrm{mm}, 800 \mathrm{~nm}$ blaze, gold coating, efficiency $\sim 80 \%$ for $\lambda=800 \mathrm{~nm}$.
} 
Princeton Instruments CCD-camera controller ST-130, which was connected to the computer via a high speed ISA card. With the $600 \mathrm{gr} / \mathrm{mm}$ grating and a $10 \mu \mathrm{m}$ wide entrance slit, the dispersion on the CCD array is $\sim 0.05 \mathrm{~nm} / \mathrm{pixel}$, and thus gave rise to a spectral resolution of $\sim 0.1 \mathrm{meV}$. The spectrometer was calibrated using an Oriel argon and helium calibration lamp. Additionally, a removable mirror was installed in front of the spectrometer, reflecting the light into another CCD camera connected with a monitor. This configuration achieveed a direct imaging of the sample surface on the monitor, thus ensuring fine alignments of the laser beam, objective, and pinhole.

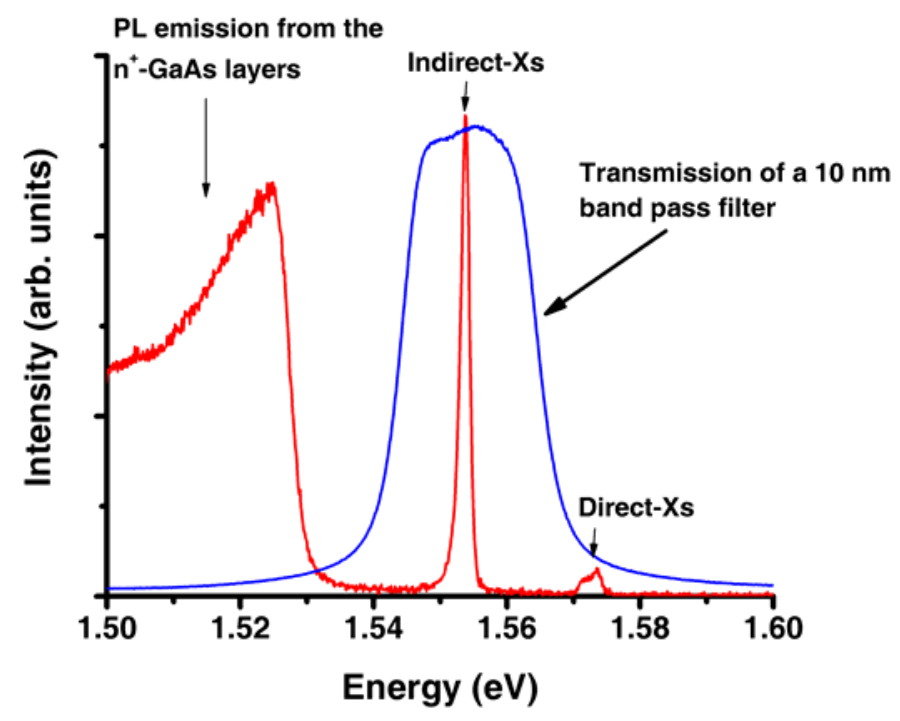

Figure 3.5 Example indirect-X PL versus transmission of a band pass interference filter used for PL imaging.

\subsubsection{Time-resolved photoluminescence}

Time-resolved PL (TRPL) or PL dynamics spectrometry was used to investigate the formation, relaxation and annihilation process of excitons in CQW. A pulse of light was directed at the sample to excite electron-hole pairs. The geminated e-h pairs or

\footnotetext{
${ }^{11}$ Princeton Instrument TEA/CCD-1024-EM/1 UV: $1024 \times 256 ; 26.6 \times 6.7 \mathrm{~mm}$ overall; $26 \times 26 \mu \mathrm{m}$ and quantum efficiency $\sim 37 \%$ for $\lambda=800 \mathrm{~nm}$.
} 
excitons typically decay back to the lower energy states by emitting photons on the order of 10-100 ns. The PL dynamics provides information on the formation and interactions of excitons.

The functional diagram for the PL dynamics spectrometer is illustrated in Figure 3.6. The beam splitter delivered a large portion of the laser pulse to the sample while simultaneously feeding a small fraction of the light pulse to a fast photodiode. The function of the photodiode is to sense "time zero", the time at which the excitation pulse hits the sample. A monochromator was inserted between the sample and the microchannel plate (MCP) detector to select the desired wavelength of PL photons from the sample.

The Time-Correlated Single Photon Counting ${ }^{12}$ - or TCSPC sampling system is capable of single photon counting. TCSPC measurements of periodic light signals provide the detection times of the individual photons and a reconstruction of the waveform [106,107]. Because of the sampling, the time resolution of TCSPC is not limited by the width of the output pulse of the detector, but by the transit-time spread or the jitter of the timing descriminator. The overall instrument response time is $\sim 30-40 \mathrm{ps,}$ which limits the time resolution available. In addition, TCSPC has a near-perfect counting efficiency and therefore achieves optimum signal-to-noise ratio for a given number of detected photons $[108,109]$.

The setup is best for photon counting rates $<50,000$ counts/s due to the deadtime limit of the multi-channel analyzer (MCA) (see Table 3.2 for a list of equipment). This can be alleviated for high counting rates by using a high rate MCA. And thus, the light

\footnotetext{
${ }^{12}$ Becker-Hickl provides excellent notes and resources for TCSPC instrumentation, see http://www.beckerhickl.com/literature.htm.
} 
intensity detected by the MCP was adjusted accordingly if the counting rates were too high.

Thus, over the range of intensities used, the MCP was able to respond to the arrival of single photons. Consequently the time interval between the laser excitation pulse and the detected PL photon randomly sampled the shape of the PL decay curve as the laser pulse was periodically repeated. The function of the remaining instrumentation in Figure 3.6 was to record and sort these time intervals to form the spectrum (see Sec.

\section{4 for TRPL measurements).}

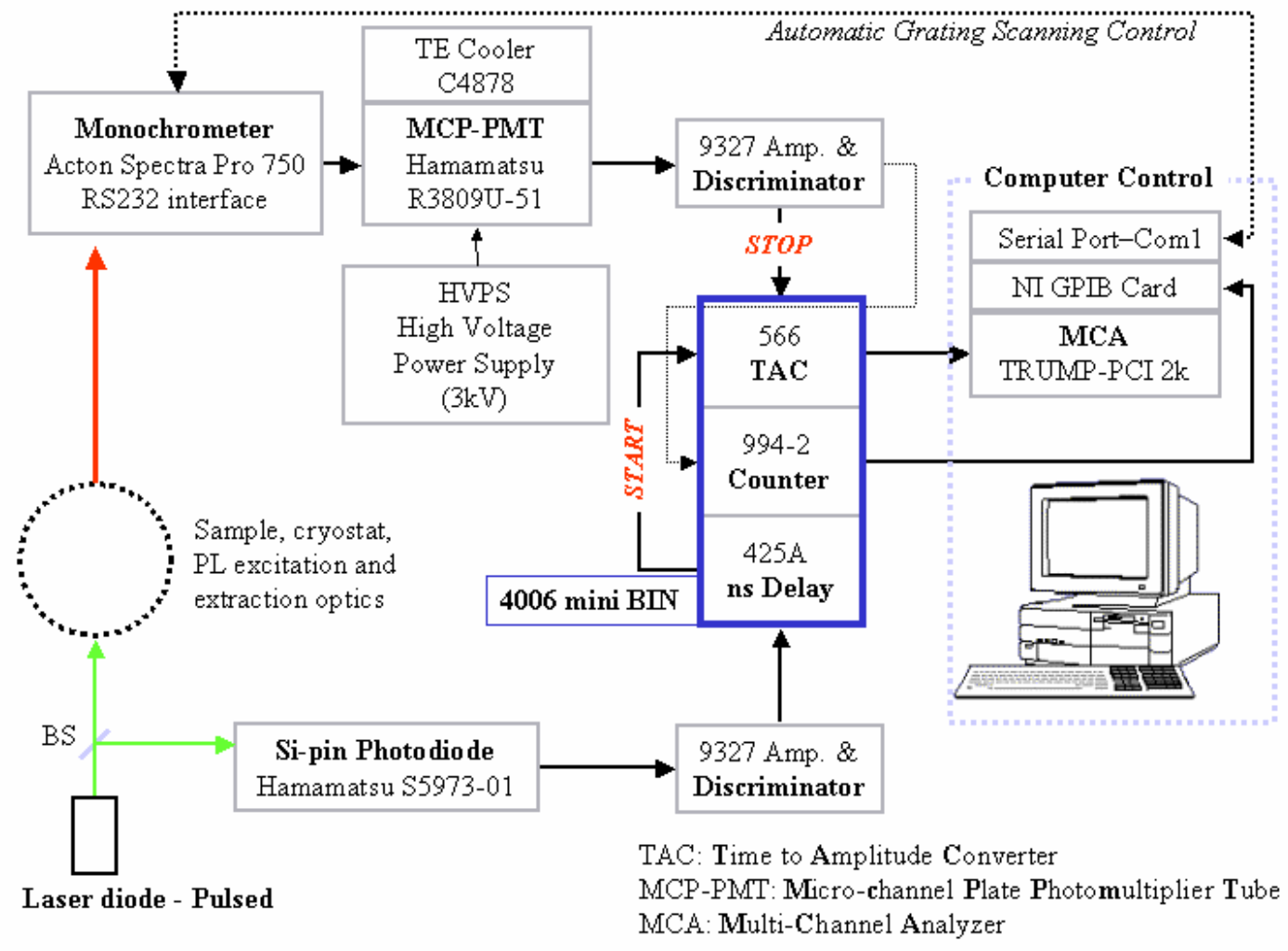

Figure 3.6 Block diagram of the time-resolved photoluminescence (TRPL) (or PL dynamics) setup with a time-correlated single photon counting (TCSPC) system. 
Table 3.2 Photoluminescence dynamics equipment list.

\begin{tabular}{|c|c|c|}
\hline Item \# & Model number & Description \\
\hline 1 & ORTEC 9327 & 1-GHz Amplifier and Timing Discriminator, jitter $<20 \mathrm{ps}$ \\
\hline 2 & ORTEC 425A & Nanosecond Delay \\
\hline 3 & ORTEC 4006 & Minibin and Power Supply \\
\hline 4 & ORTEC 566 & Time-to-Amplitude Converter \\
\hline 5 & $\begin{array}{l}\text { ORTEC } \\
\text { TRUMP-PCI-2k }\end{array}$ & $\begin{array}{l}\text { TRUMP-PCI MCA for Windows }(8-\mu \text { s conversion time, } 2 \mathrm{k} \\
\text { channels) (include MASESTRO }{ }^{\mathrm{TM}}-32 \text { ) }\end{array}$ \\
\hline 6 & R3809U-51 & $\begin{array}{l}\text { Micro-Channel Plate Photomultiplier Tube (Hamamatsu) } \\
\text { Qantum efficiency } 5 \% \text { at } 800 \mathrm{~nm} \text {, transit-time spread }<25 \mathrm{ps} \text {. }\end{array}$ \\
\hline & C4878 & Thermoelectric Cooler for MCP (Hamamatsu) \\
\hline 7 & S5973-01 & Si-pin fast photodiode: Cut-off $1.2 \mathrm{GHz}$ (Hamamatsu) \\
\hline 8 & & Pulsed lasers (laser diodes in the pulsed modes) \\
\hline
\end{tabular}

\subsection{Electrical properties: Photocurrent}

The exciton gas was subject to a transverse (cross-well) static electric field by applying a bias to the $n+-i-n+$ CQW samples with a DC precision voltage source. The stationary photocurrent was measured by an HP4140B pA meter.

Under pulsed laser excitations, photocurrent transients were converted to voltage transients by a transpedence preamplifier and measured by a digital oscilloscope which can be read out through the GPIB interface by a computer. With a combination of a 100 $\mathrm{MHz}$ transpedence (current-to-voltage) preamplifier $^{13}$ and $500 \mathrm{MHz}$ digital oscilloscope $^{14}$, a time resolution of $\sim 10 \mathrm{~ns}$ was achieved for the photocurrent transient measurements (Figure 3.7).

\footnotetext{
${ }^{13}$ Femto HCA-100M-50K-C: $100 \mathrm{MHz}$, Gain=50K V/A.

${ }^{14}$ LeCroy Waverunner LT344 500MHz digital oscilloscope: Maximum effective sampling rate is $500 \mathrm{MS} / \mathrm{s}$ in single-shot mode and $25 \mathrm{GS} / \mathrm{s}$ in random interleaved sampling mode.
} 


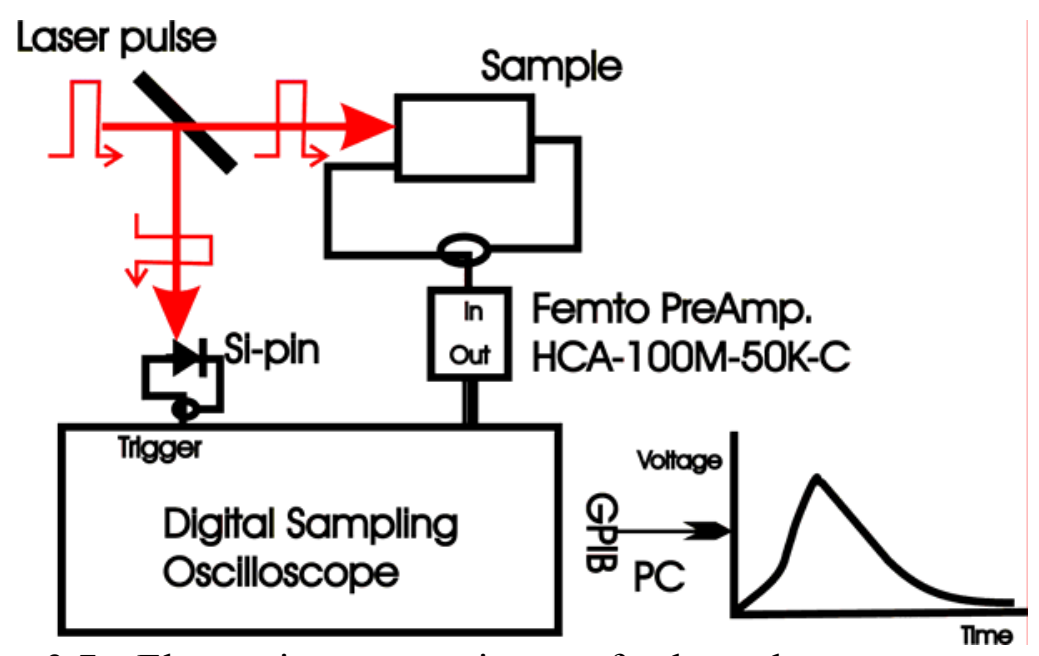

Figure 3.7 Electronic connections of the photocurrent transient measurements. 


\section{Chapter 4 Spatially Indirect Excitons}

\subsection{Introduction}

In this chapter, the exciton states in coupled quantum wells (CQW) are first described. Then the properties of the spatially indirect excitons (indirect-Xs) through time-integrated and time-resolved photoluminescence (PL) measurements as a function of bias voltage $\left(V_{b}\right)$, temperature $(T)$ and laser excitation power or intensity ( $\left(P_{\text {laser }}\right.$ or $\left.I_{\text {laser }}\right)$ are detailed.

\subsection{Exciton states in coupled quantum wells}

The Hamiltonian for a nearly free electron-hole pair confined to potentials $\mathrm{V}_{\mathrm{e}}$ and $\mathrm{V}_{\mathrm{h}}$ by the quantum wells in the presence of an external static electric field $\vec{F}$ is given by

$$
\begin{aligned}
\hat{H}= & -\frac{\hbar^{2}}{2 m_{e}} \nabla_{e}^{2}-\frac{\hbar^{2}}{2}\left(\frac{1}{m_{h \|}} \nabla_{h \|}^{2}+\frac{1}{m_{h \perp}} \frac{\partial^{2}}{\partial z_{h}^{2}}\right)-\frac{e^{2}}{\varepsilon\left|\vec{r}_{e}-\vec{r}_{h}\right|} \\
& +V_{e}\left(\vec{r}_{e}\right)+V_{h}\left(\vec{r}_{h}\right)-e \vec{F} \cdot\left(\vec{r}_{e}-\vec{r}_{h}\right)+E_{g}
\end{aligned}
$$

where $\vec{r}_{e(h)}=\left(x_{e(h)}, y_{e(h)}, z_{e(h)}\right)$ denotes the electron (hole) coordinates. The Hamiltonian can be recasted into the relative coordinates $(\vec{r})$ and center of mass $(\mathrm{CM})$ coordinates $(\vec{R})$ according to

$$
\begin{aligned}
& \vec{r}=(x, y, z)=\left(x_{e}-x_{h}, y_{e}-y_{h}, z_{e}-z_{h}\right), \text { and } \\
& \vec{R}=(X, Y, Z)=\left(\frac{m_{e} x_{e}+m_{h \|} x_{h}}{m_{e}+m_{h \|}}, \frac{m_{e} y_{e}+m_{h \|} y_{h}}{m_{e}+m_{h \|}}, \frac{m_{e} z_{e}+m_{h \perp} z_{h}}{m_{e}+m_{h \perp}}\right) .
\end{aligned}
$$


The confining potential in the quantum wells depends on coordinate $\mathrm{z}$ only (the growth direction). If the static electric is applied in the z-direction as well, the Hamiltonian can be simplified in the relative and $\mathrm{CM}$ coordinates as

$$
\begin{aligned}
\hat{H} & =-\frac{\hbar^{2}}{2 m_{e}} \frac{\partial^{2}}{\partial z_{e}{ }^{2}}+V_{e}\left(z_{e}\right)-e F z_{e}-\frac{\hbar^{2}}{2 m_{h \perp}} \frac{\partial^{2}}{\partial z_{h}{ }^{2}}+V_{h}\left(z_{h}\right)+e F z_{h} \\
& -\frac{\hbar^{2}}{2 \mu_{\|}}\left(\frac{\partial^{2}}{\partial x^{2}}+\frac{\partial^{2}}{\partial y^{2}}\right)-\frac{\hbar^{2}}{2 M_{\|}}\left(\frac{\partial^{2}}{\partial X^{2}}+\frac{\partial^{2}}{\partial Y^{2}}\right)-\frac{e^{2}}{\varepsilon \sqrt{x^{2}+y^{2}+\left(z_{e}-z_{h}\right)^{2}}}+E_{g}
\end{aligned}
$$

where $\mu_{\|}=\left(m_{e}^{-1}+m_{h \|}^{-1}\right)^{-1}$ and $M_{\|}=m_{e}+m_{h \|}$ are reduced and total masses of the exciton respectively.

The electron effective masses in GaAs and $\mathrm{Al}_{0.33} \mathrm{Ga}_{0.67} \mathrm{As}$ are given empirically [51] as

$$
\begin{array}{lll}
\text { GaAs } & m_{e} & =0.067 m_{0} \\
\mathrm{Al}_{0.33} G a_{0.67} \text { As } & m_{e} & =0.067+0.0838 \times 0.33 \quad m_{0} \\
& & =0.0947 m_{0} .
\end{array}
$$

The effective heavy-hole (HH) masses given by Eq. (2.1) and (2.2) with the empirical Luttinger parameters for $\mathrm{GaAs}$ and $\mathrm{Al}_{\mathrm{x}} \mathrm{Ga}_{1-\mathrm{x}} \mathrm{As}$ are as follows:

\begin{tabular}{|l|l|l|}
\hline Direction & GaAs: $\mathrm{m}_{\mathrm{hh}} / \mathrm{m}_{0}$ & $\mathrm{Al}_{\mathrm{x}} \mathrm{Ga}_{1-\mathrm{x}} \mathrm{As}(\mathrm{x}=0.33): \mathrm{m}_{\mathrm{hh}} / \mathrm{m}_{0}$ \\
\hline$[001]: \|$ & 0.11 & $0.11+0.10 \mathrm{x}=0.143$ \\
\hline$[001]: \perp$ & 0.33 & $0.33+0.18 \mathrm{x}=0.169$ \\
\hline
\end{tabular}

Using an empirical conduction-band offset ratio $\mathrm{Q}_{\mathrm{c}} \equiv \Delta \mathrm{E}_{\mathrm{d}} / \Delta \mathrm{E}_{\mathrm{g}} \approx 0.65$, we obtain $\Delta \mathrm{E}_{\mathrm{c}} \approx 0.274 \mathrm{eV}$ and $\Delta \mathrm{E}_{\mathrm{v}} \approx 0.148 \mathrm{eV}$ for GaAs/ $/ \mathrm{Al}_{0.33} \mathrm{Ga}_{0.67} \mathrm{As}$ heterostructures (band gaps $\mathrm{E}_{\mathrm{g}}(\mathrm{GaAs})=1.519 \mathrm{eV}$ and $\left.\mathrm{E}_{\mathrm{g}}\left(\mathrm{Al}_{0.33} \mathrm{Ga}_{0.67} \mathrm{As}\right)=1.84 \mathrm{eV}\right)$. The eigenstates, including bound and free electron-hole pair eigenstates, of the Hamiltonian can then be solved with these given values. Note that the Hamiltonian given above is for a single exciton without considering the spin or many-body interaction of excitons [110]. The energy levels, 
binding energy, Bohr radius, and oscillator strength (lifetime) of excitons in CQW have been calculated by variational methods [111-118]. In ref. [119], Szymanska et al. calculated these parameters for the 8-4-8nm GaAs/AlGaAs CQW structures studied here. Tunneling and relaxation processes in CQW have also been studied [120,90]. Here we focus on properties of spatially indirect-Xs based on PL spectroscopic experiments.

\subsection{Time-integrated photoluminescence spectroscopy}

This section describes time-integrated photoluminescence of the CQW as a function of the bias voltage $\left(V_{b}\right)$, temperature $(T)$, and excitation intensity $\left(P_{\text {laser }}\right)$ and energy $(h v)$. The studied CQW sample has an 8-4-8-nm GaAs/AlGaAs active region and an overall $n^{+}-i-n^{+}$structure (see Table 3.1 for the complete structure). A bias voltage $V_{b}$ was applied between the metallic $\mathrm{n}^{+}$-layers. The electric field is approximated as $F=V_{b} / W_{i}$, where $W_{i}=420 \mathrm{~nm}$ is the total thickness of the intrinsic insulating layer (neglecting the screening effect of photogenerated carriers).

The exciton $(\mathrm{X})$ PL spectral lines $\left[S_{X}(E)\right]$ are typically characterized by the peak intensity $\left(\mathrm{I}_{0}\right)$, peak energy $\left(\mathrm{E}_{0}\right)$, and linewidth (Full-Width-at-Half-Maxima, $Г$ ). To further analyze the X PL spectra, we introduce the central moments and associated cumulants $[121,122] .{ }^{15}$

The central moments of $S_{X}(E)$ are given by:

$$
\mu_{n}=\left\langle(E-\langle E\rangle)^{n}\right\rangle=\int(E-\mu)^{n} S_{X}(E) d E,
$$

where $\langle E\rangle$ denotes the expectation value.

And the cumulants can be expressed in terms of the central moments as:

\footnotetext{
${ }^{15}$ See also Worlfram Research (http://mathworld.wolfram.com/Moment.html) or Numerical Recipes Home Page (http://www.nr.com/).
} 


$$
\kappa_{1}=\mu ; \quad \kappa_{2}=\mu_{2} ; \quad \kappa_{3}=\mu_{3} ; \quad \kappa_{4}=\mu_{4}-3 \mu_{2}^{2}
$$

where $\mu$ is the mean and $\sigma^{2} \equiv \mu_{2}$ is the variance. $S_{X}(E)$ can also be characterized by the reduced cumulants $k_{n} \equiv \kappa_{n} / \sigma^{n}$, in particular the skewness $s=k_{3}$ (asymmetry) and the kurtosis $k=k_{4}$ (the concentration peakedness as compared to a Gaussian distribution). Table 4.1 lists the spectral characteristics of X PL and corresponding symbols.

Table 4.1 List of symbols characterizing exciton photoluminescence.

\begin{tabular}{|l|l|}
\hline Characteristics of X PL & Symbols \\
\hline Peak intensity & $\mathrm{I}_{0}$ \\
\hline Peak energy & $\mathrm{E}_{0}$ \\
\hline Linewidth (FWHM) & $\Gamma$ \\
\hline Integrated intensity: $\mu_{0}$ & $\mathrm{I}_{\mathrm{X}}$ \\
\hline Mean energy: $\mu$ & $\mathrm{E}_{\mathrm{X}}$ \\
\hline Standard variation: $\sigma$ & $\sigma_{\mathrm{X}}$ \\
\hline Skewness/Asymmetry: $s$ & $\mathrm{~A}_{\mathrm{X}}$ \\
\hline Kurtosis: $k$ & $\mathrm{~K}_{\mathrm{X}}$ \\
\hline
\end{tabular}

\subsubsection{Electric field dependence}

In the absence of an external electric field, the optical transition with lowest energy is the direct exciton (direct-X) recombination. By tuning the energy levels of the two wells by an electric field $(F)$, the spatially indirect-Xs become the lowest-energy interband excitation. Figure 4.1A-B are the PL spectra at $\mathrm{T}=1.7 \mathrm{~K}$ excited with a uniform HeNe laser $\left(P_{\text {laser }}=2711 \mu \mathrm{W}\right.$ and spot radius $\sigma_{\mathrm{r}}=130 \mu \mathrm{m}$, corresponding to an excitation intensity $\left.I_{\text {laser }} \approx P_{\text {laser }} /\left(2 \pi \sigma_{\mathrm{r}}^{2}\right) \approx 2.5 \mathrm{~W} / \mathrm{cm}^{-2}\right)$ for bias voltages $V_{b}=-1.5 \rightarrow+1.5$ V. The PL intensities of direct-X (inter-well) transitions are one to two orders of magnitude lower than those of indirect-Xs for $V_{b}>0.1 \mathrm{~V}$. Figure $4.1 \mathrm{C}$ shows the peak energies of excitons or trions (three-particle charge complexes) [123-126]. The direct-X 
peak energy is almost constant up to $V_{b}=1.5 \mathrm{~V}(F \approx 36 \mathrm{kV} / \mathrm{cm})$, while as the indirect-X peak shifted more than $30 \mathrm{meV}$ (Figure 4.1C).
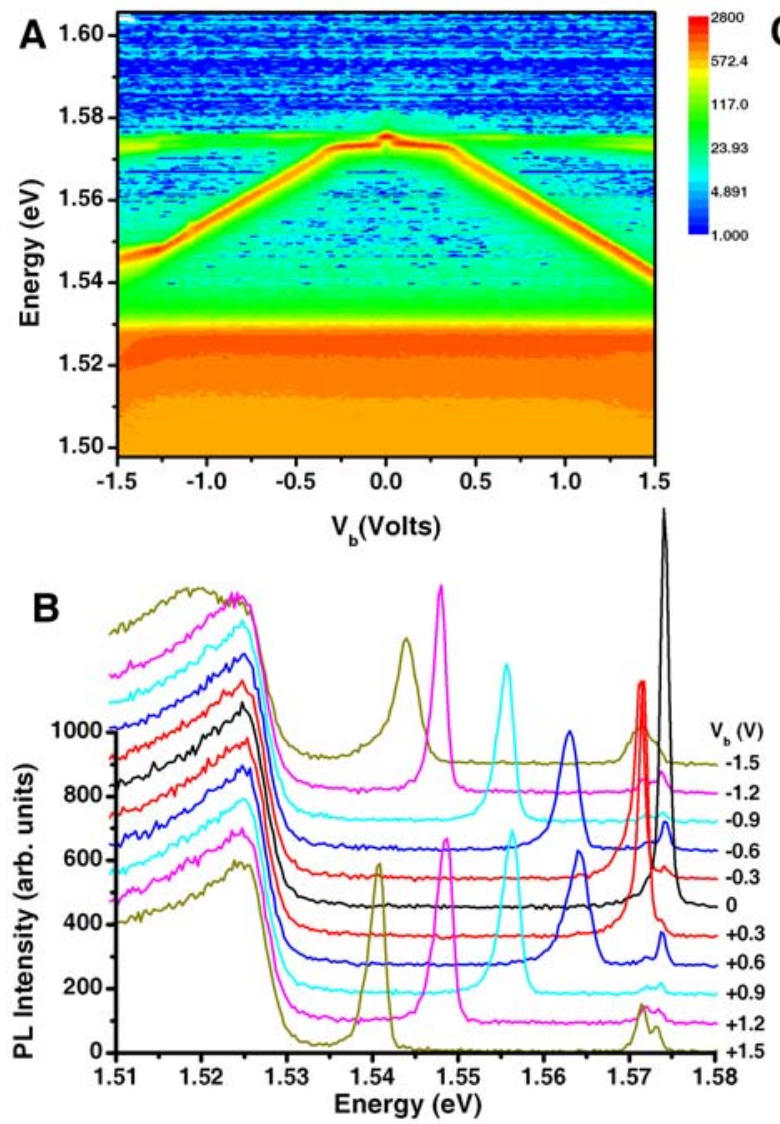
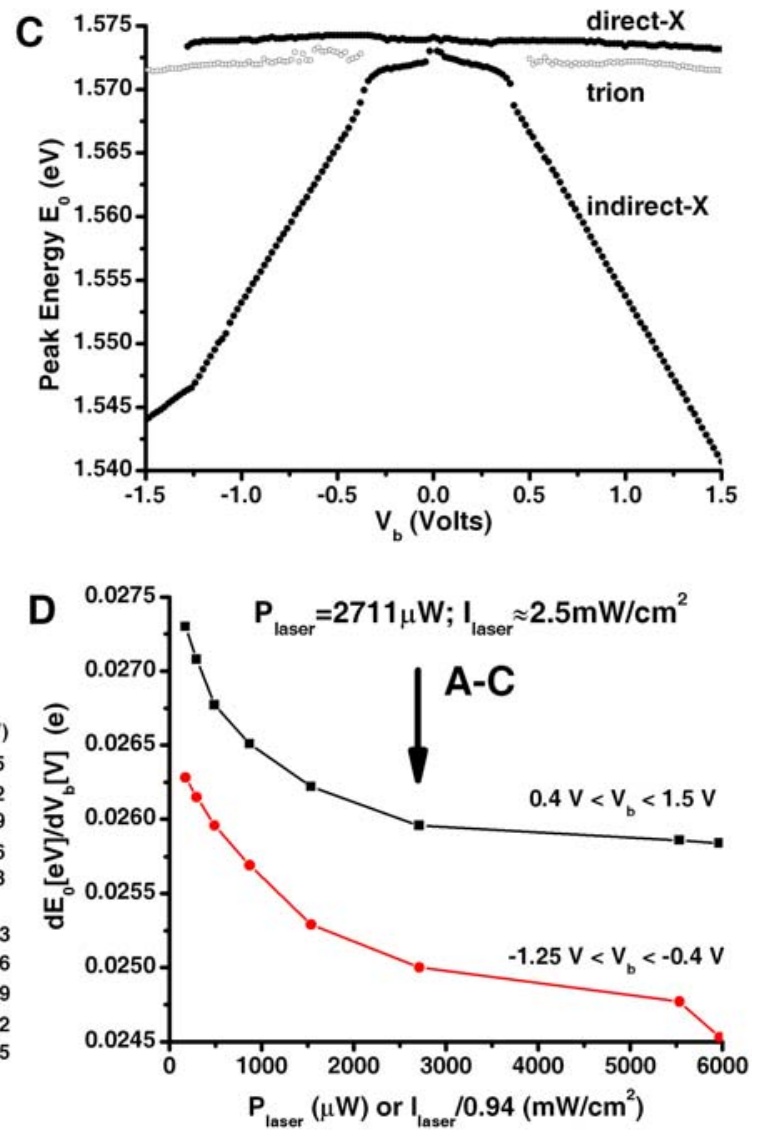

Figure $4.1 V_{b}$-dependent PL spectra and analysis. (A) PL spectra versus $V_{b}$. (B) Selected $V_{b}$-dependent spectra. (C) Peak energies of Xs or trions versues $\mathrm{Vb}$. (D) Derivitives of $\mathrm{E}_{0}\left(V_{b}\right)$ with data for the specified range. .

The X PL peak is assigned to a transition involving an electron and a heavy-hole with peak energy $\mathrm{E}_{0} \approx \mathrm{E}_{\mathrm{g}}+\mathrm{E}_{\mathrm{e} 1}+\mathrm{E}_{\mathrm{hh} 1}-\mathrm{E}_{\mathrm{b}}$, where $\mathrm{E}_{\mathrm{g}}$ is the GaAs bandgap, $\mathrm{E}_{\mathrm{e} 1}$ and $\mathrm{E}_{\mathrm{hh} 1}$ the first electron and heavy-hole subbands, and $\mathrm{E}_{\mathrm{b}}$ the $\mathrm{X}$ binding energy (see also transition $\# 2$ in Figure 2.1). The $V_{b}$-dependent indirect-X energy is quadratic-like at low fields $(\approx$ flat-band conditions) and become linear at higher fields $\left(\left|\mathrm{V}_{\mathrm{b}}\right|>0.3 \mathrm{~V}\right.$ here), in agreement with theory [80]. 
The electric field dependence of the exciton binding energy $\left(E_{b}\right)$ is more than an order of magnitude weaker that that of the X peak/average energy. Binding energies of indirect-Xs in the GaAs/AlGaAs CQW structure used have been determined by exact solutions of the Schrödinger equation in a certain basis within the anisotropic effective mass approximation [119]. The calculated $\mathrm{E}_{\mathrm{b}}$ is almost constant $(\sim 4.2 \mathrm{meV})$, for $F=5$ $40 \mathrm{kV} / \mathrm{cm}$. Therefore, the change of $\mathrm{E}_{\mathrm{b}}$ with the field can be neglected for high fields. $\mathrm{E}_{\mathrm{el}}$ and $E_{\text {hhl }}$ follow the shift of the Fermi energy in the presence of the electric fields, thus the peak energy $\mathrm{E}_{0}$ shift linearly with $F$. This shift $\left(\Delta \mathrm{E}_{0}\right)$ can be approximated by

$$
\Delta E_{0} \approx e F d \approx e \frac{f \times V_{b}}{W_{i}} d
$$

where $F$ is the electric field, $d$ the average separation of e-h in the z-direction, $W_{i}$ the thickness of the insulating intrinsic region $\left(W_{i}=420 \mathrm{~nm}\right)$, and $f$ the fraction of $V_{b}$ dropping across $W_{i}$. When the bias voltage drops completely across the intrinsic region (i.e. perfect ohmic contacts with negligible junction gap or screening due to photocarriers or space charges), $f=1$. The deviation from this ideal situation can be significant and $f$ can be much less than 1 . The average separation between electron and hole layers, $d$, is almost independent of $F[119]$; therefore, the slope of the linear shift of $\mathrm{E}_{0}$ with $V_{b}$ $\left(d E_{0} / d V_{b}=f \times e d / W_{i}\right)$ is a better indicator of the effective electric field in the coupled quantum wells active region. As seen in Figure 4.1D, $d E_{0} / d V_{b}$ decreases with increasing laser excitation power $\left(P_{\text {laser }}\right)$, which indicates a reducing electric field $F$. The reduction in $F$ can be attributed to non-ideal ohmic contacts and/or screening by photogenerated carriers. Using the value of $d E_{0} / d V_{b}$ under a low $P_{\text {laser }}$ and positive bias where screening effects are negligible ( $\mathrm{f} \approx 1$ ), we deduced $d \approx \frac{d E_{0}[e V]}{e \cdot d V_{b}[V]} \times W_{i}=0.02573 \times 420 \mathrm{~nm}=11.5 \mathrm{~nm}$, 
close to the as grown average distance between the centers of the adjacent coupled quantum wells. Note that for $V_{b}<0$, the photocurrent is significantly higher and exhibits a negative differential resistance (NDR) region (see Figure 7.16) though the CQW sample was designed to be symmetric and undoped. This signals cross-well transport of carriers and screening of the applied electric fields. We defer further discussions on these effects to Chapter 7

When the bias is increased, the integrated indirect-X PL intensity $\left(\mathrm{I}_{\mathrm{x}}\right)$ can be quenched (Figure 4.1B), indicative of non-negligible nonradiative recombination. The applied electric field reduces the e-h overlap, leading to a long optical recombination time; therefore, nonradiative recombination channels can take over. A constant X PL intensity versus $V_{b}$ would thus indicate a high quality quantum well sample with negligible nonradiative recombinations. However, under HeNe lasers $(\mathrm{h} v=1.959 \mathrm{eV})$ where carriers are generated in the AlGaAs barrier layers, X PL intensity can depend strongly on $V_{b}$ due to the carriers transport across and capture by quantum wells $[127,128]$. We will address this aspect of carrier transport more fully in Chapter 7.

\subsubsection{Excitation intensity dependence}

\subsubsection{Local excitation}

The PL spectra were measured through a pinhole (effective diameter $\phi \approx 3 \mu \mathrm{m}$ ) centered at the excitation spot in the first confocal image plane under a tightly focused HeNe laser excitating the CQW sample at $T=1.7 \mathrm{~K}$. The laser excitation spot has a Gaussian intensity profile with a radius $\sigma_{\mathrm{r}} \approx 6.1 \mu \mathrm{m}$, giving an excitation intensity $\left(I_{\text {laser }}\right)$ approximately as $I_{\text {laser }}=P_{\text {laser }} /\left(2 \pi \sigma_{r}^{2}\right)$. 

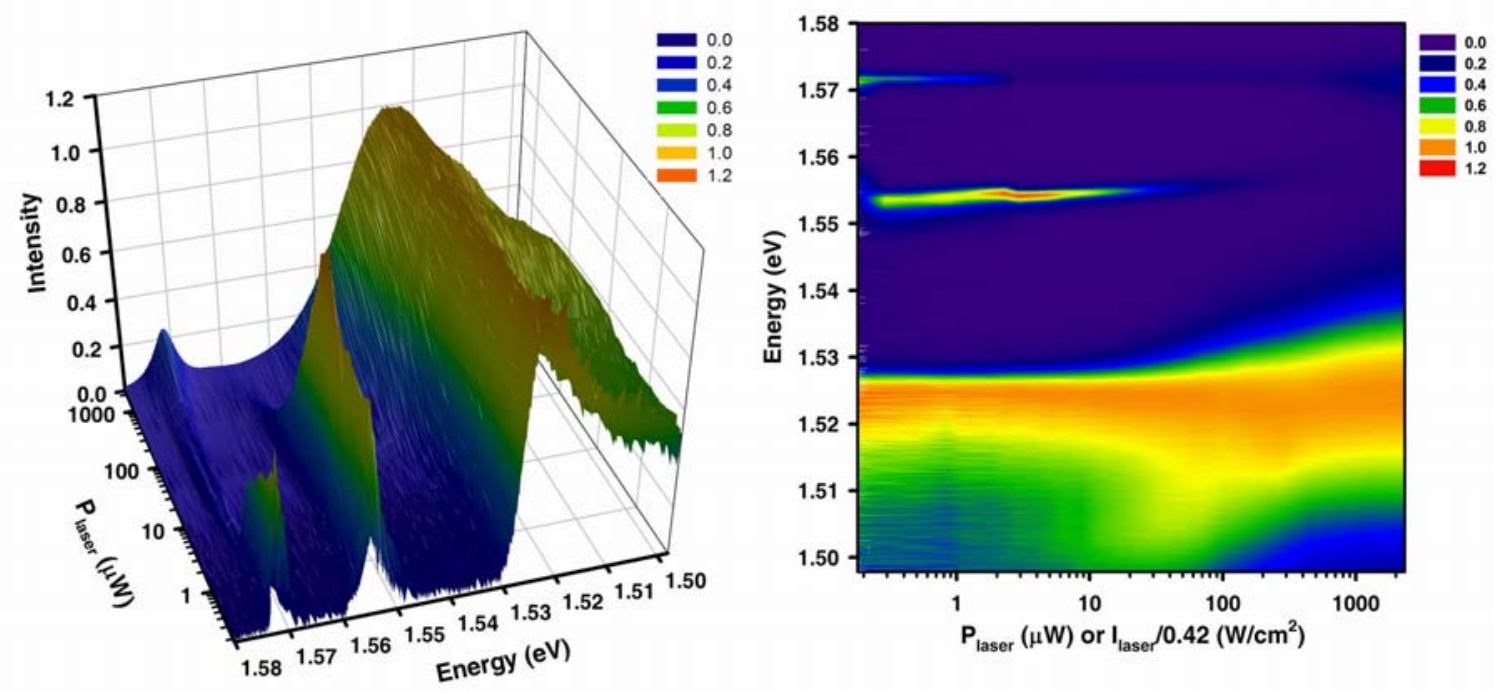

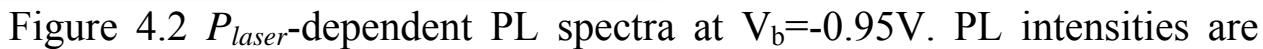
rescaled by normalizing peak $\mathrm{n}^{+}$-GaAs PL intensities. (HeNe laser excitation: spot radius $\sigma \approx 6.1 \mu \mathrm{m}, \mathrm{h} \nu=1.959 \mathrm{eV}$, and $I_{\text {laser }} \approx$ $0.42 \times P_{\text {laser }}[\mu \mathrm{W}] \mathrm{W} / \mathrm{cm}^{-2}$.)

Figure 4.2 shows the measured PL spectra at $V_{b}=-0.95 \mathrm{~V}$ with substrate $\mathrm{n}^{+}$-GaAs PL $\left(\mathrm{n}^{+}-\mathrm{PL}\right)$ normalized to one as a function of $P_{\text {laser }}$. When $P_{\text {laser }}$ is increased, the indirectX PL intensity $\left(\mathrm{I}_{0}\right)$ first grows linearly before saturates at $P_{\text {laser }} \approx 100 \mu \mathrm{W}$ (Figure 4.3B). In addition, the peak intensity of indirect-Xs becomes comparably less than that of directXs. This could be due to ionization of indirect-Xs, heating effects or the increasing importance of the underlying Fermi statistics of constituent electrons and holes of Xs. The saturation occurs at an $\mathrm{X}$ density $\sim 10^{10} \mathrm{~cm}^{-2}$ (see Sec. 4.5 for $\mathrm{X}$ density estimation), less than the Mott transition $[129,130]$ density $\left(\sim 2 \times 10^{11} \mathrm{~cm}^{-2}\right)$ using an $\mathrm{X}$ Bohr radius $\mathrm{a}_{\mathrm{X}}$ $=12 \mathrm{~nm}$. With increasing $P_{\text {laser }}$, the linewidth $(\Gamma)$ decreases and then broadens for intensities near for which the PL intensity deviates from the linear growth with $P_{\text {laser }}$ (Figure 4.3). In the low-density regime, the broadening is attributed to inhomogeneous broadening caused by disorder; whereas in the high-density regime, it can be caused by the presence of free carriers from ionized excitons. In the moderate density regime, $\Gamma$ is a 
minimum, where interface disorder is possibly screened by the repulsive interactions among Xs[39]. Note that a model describing an unbalanced migration of photogenerated charge carriers was also proposed to explain the linewidth reduction observed in a different system [131].

Due to the dipolar repulsive interaction between spatially indirect-Xs, the $\mathrm{X}$ energy is expected to increase (blue shift) linearly with $X$ density $n_{X}$ $\left(\delta E_{0}=E_{0}\left(n_{X}\right)-E_{0}(0) \propto n_{X}\right)$ under mean field approximations [132,19,110]. On the contrary, the $\mathrm{X}$ peak energy $\left(\mathrm{E}_{0}\right)$ versus $P_{\text {laser }}$ in Figure 4.4A shows a nonlinear increase with a weak red shift at moderate $P_{\text {laser }}$ Nevertheless, the energy shift $\delta$ E can be used as an indicator of $\mathrm{X}$ density especially for the low-density regime. This will be addressed in more details in Sec. 4.5.

Finally, we discuss the $\mathrm{n}^{+}-\mathrm{PL}$, which shows an excellent linear dependence on $P_{\text {laser }}$ over a wide range ( four orders of magnitude) (Figure $\left.4.3 \mathrm{~B}\right)$. This confirms the performance of PL extraction and detection of the optical system and accurate relative power measurements. In addition, $\mathrm{n}^{+}-\mathrm{PL}$ shifts to high energy at $P_{\text {laser }}>100 \mu \mathrm{W}$ (or $I_{\text {laser }}$ $>40 \mathrm{~W} / \mathrm{cm}^{-2}$ ), which signals high density photogenerated carriers in the substrate (Figure 4.2B). 

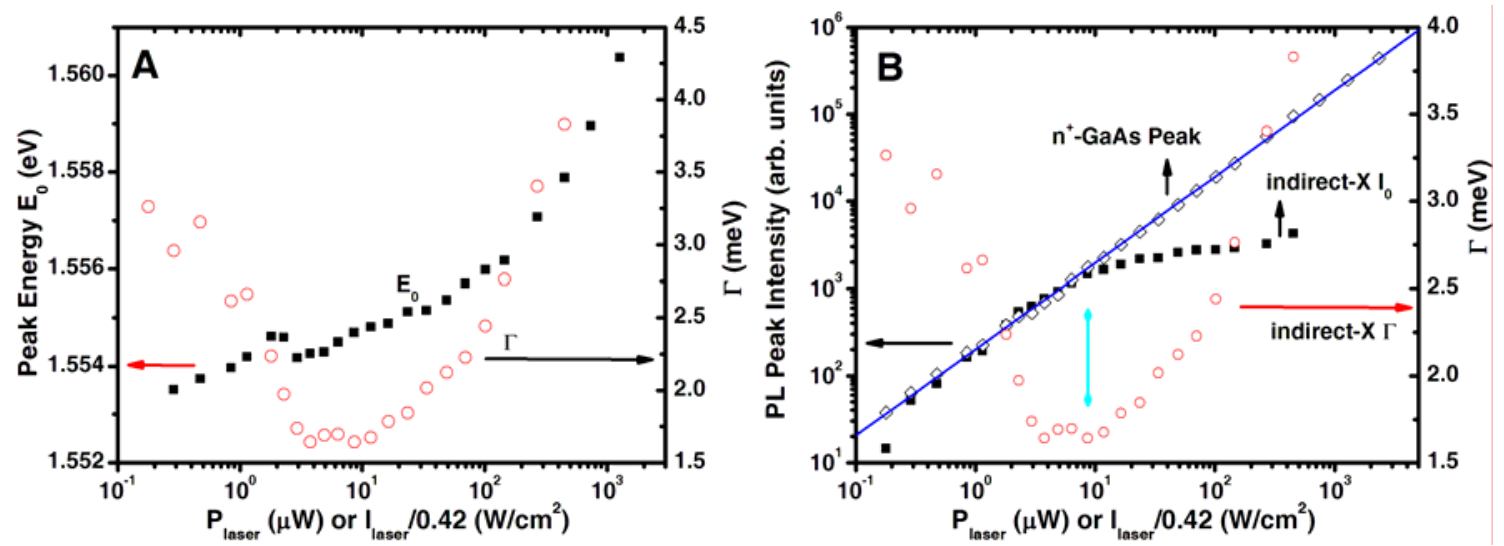

Figure 4.3 $P_{\text {laser }}$ dependent PL analysis at $V_{b}=-0.95 \mathrm{~V}$ and $T=1.7 \mathrm{~K}$. (HeNe laser excitation: spot radius $\sigma_{\mathrm{r}} \approx 6.1 \mu \mathrm{m}, \mathrm{h} v=1.959 \mathrm{eV}$, and $I_{\text {laser }} \approx$ $0.42 \times P_{\text {laser }}[\mu \mathrm{W}] \mathrm{W} / \mathrm{cm}^{-2}$.)

\subsubsection{Uniform excitation}

The indirect-X PL spectra discussed previously come from the central excitation spot (within a diameter $<3 \mu \mathrm{m}$ ) where electron-hole (e-h) pairs (photocarriers) are directly generated and heating of the lattice can occur. Moreover, after the generation of e-h pairs or formation of Xs, relaxation in the momentum space (energy) and transport in real space occur at the same time. To reduce the spatial dependence or heating effects, we measured the $V_{b}$-dependent PL spectra at a locality $(<3 \mu \mathrm{m}$ square $)$ under a uniform excitation of a spot radius $\sigma_{\mathrm{r}} \approx 130 \mu \mathrm{m}$.

We make the following observations: (i) Indirect-X peak $\left(\mathrm{I}_{0}\right)$ and $\mathrm{n}^{+}$-GaAs PL intensities grows linearly with increasing $P_{\text {laser }}$ (Figure 4.4), which corresponds to the low density regime under a tightly focused excitation. (ii) The $\mathrm{X}$ energy shifts approximately linearly for $I_{\text {laser }}<2 \mathrm{~W} / \mathrm{cm}^{-2}$, but deviates from the linear trend for high excitation intensities (Figure 4.4), consistent with the results under a local excitation spot. (iii) The X PL peaks possess asymmetric shapes with low energy tails, which can be seen from the $\sim 0.5 \mathrm{meV}$ lower energy of $\mathrm{E}_{\mathrm{X}}$ than $\mathrm{E}_{0}$ (Figure 4.4) and negative skewness $\left(\mathrm{S}_{\mathrm{X}}\right)$ (not shown). 


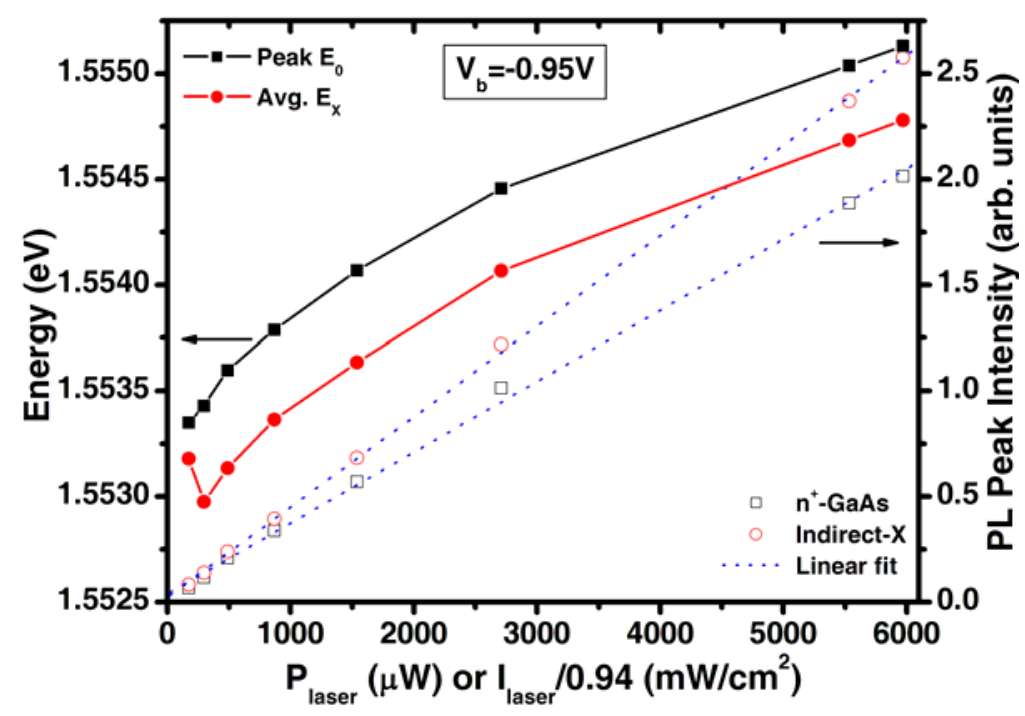

Figure 4.4 $P_{\text {laser }}$ dependent exciton PL energies and peak intensities at $V_{b}=$ $-0.95 \mathrm{~V}$ under a uniform HeNe excitation. Solid squares: Peak $\mathrm{X}$ energy $\left(E_{0}\right)$. Solid circules: Averagy $X$ energy $\left(E_{x}\right)$. Open squares: Peak PL intensities from $\mathrm{n}+-\mathrm{GaAs}\left(\mathrm{n}^{+}-\mathrm{PL}\right)$. Open circles: indirect-X PL peak intensity. Note that $P_{\text {laser }}$ is in the linear scale as compared to the log scale in Figure 4.3.

\subsection{Time-resolved photoluminescence}

This section describes the time-resolved photoluminescence (TRPL) of spatially indirect-Xs versus the bias voltages $\left(V_{b}\right)$, excitation intensity $\left(P_{\text {laser }}\right)$, and temperature $(T)$. A pulsed diode laser of $h v=1.944 \mathrm{eV}(\lambda=637.8 \mathrm{~nm})$ was tightly focused onto the CQW sample to simulate the excitation conditions at a HeNe laser $(\mathrm{h} v=1.959 \mathrm{eV})$. For nonresonant excitation above the conduction band edge of the AlGaAs barrier, the excess photogenerated carriers quickly diffuse and thermalize to the lowest bound states in the well region. This contributes to the observed rise time. The decay time reflects radiative and non-radiative recombination in the well and possible carrier tunneling in and out of the wells.

TRPL has been widely assumed to provide a direct measurement of the radiative lifetime of a given transition. In fact, the situation is usually more complicated due to 
formation, relaxation, and transport processes of Xs, and the presence of nonradiative recombination. Nevertheless, the X PL decay can be approximated as a simple exponential decay under certain conditions. In this case, the observed rate of luminescent photo-emission is given by: $\mathrm{R}=\left(\mathrm{N}_{0} / \tau\right) \mathrm{e}^{\mathrm{t} / \tau}$, where $\mathrm{R}$ is the rate in photons/s, $\mathrm{N}_{0}$ the number of Xs excited by the light pulse, $t$ the observed time of photon emission after excitation, and $\tau$ the characteristic decay time constant of Xs. The logarithm of the observed emission rate can be plotted versus time to obtain a straight line, whose slope is $-1 / \tau$. This defines approximately the radiative lifetime of Xs if nonradiative recombination is negligible.

As mentioned previously in Sec. 2.4.1.1, electric fields in the z-direction transform a strong symmetry-allowed transition for the flat-band condition into a weak one [82]. The main transition in PL is the e1-hh1 transition as seen in Figure 2.1. For this spatially "indirect" (inter-well) transition, the separation of the electron and hole wave functions increases with bias. Thus the oscillator strength is reduced and the lifetime increased. In contrast, the intra-well transition 2 (Figure 2.1), which is symmetry forbidden for a flat-band, becomes dipole allowed but remains spatially direct under an electric field. This direct-X transition is thus expected to have a fast PL transient. In the 8-4-8 $\mathrm{nm} \mathrm{GaAs} / \mathrm{AlGaAs} \mathrm{CQW}$ studied, $\tau$ typically falls in the range of a few $100 \mathrm{ps}$ for direct-Xs and up to $\approx 100 \mathrm{~ns}$ for spatially indirect-Xs under $\sim 30 \mathrm{kV} / \mathrm{cm}$ electric fields. The value of $\tau$ of indirect-Xs is primarily determined by the applied electric field, but it is also affected by the localization, temperature, exciton density, and the presence of free carriers. 


\subsubsection{Spatially- and time-resolved photoluminescence}

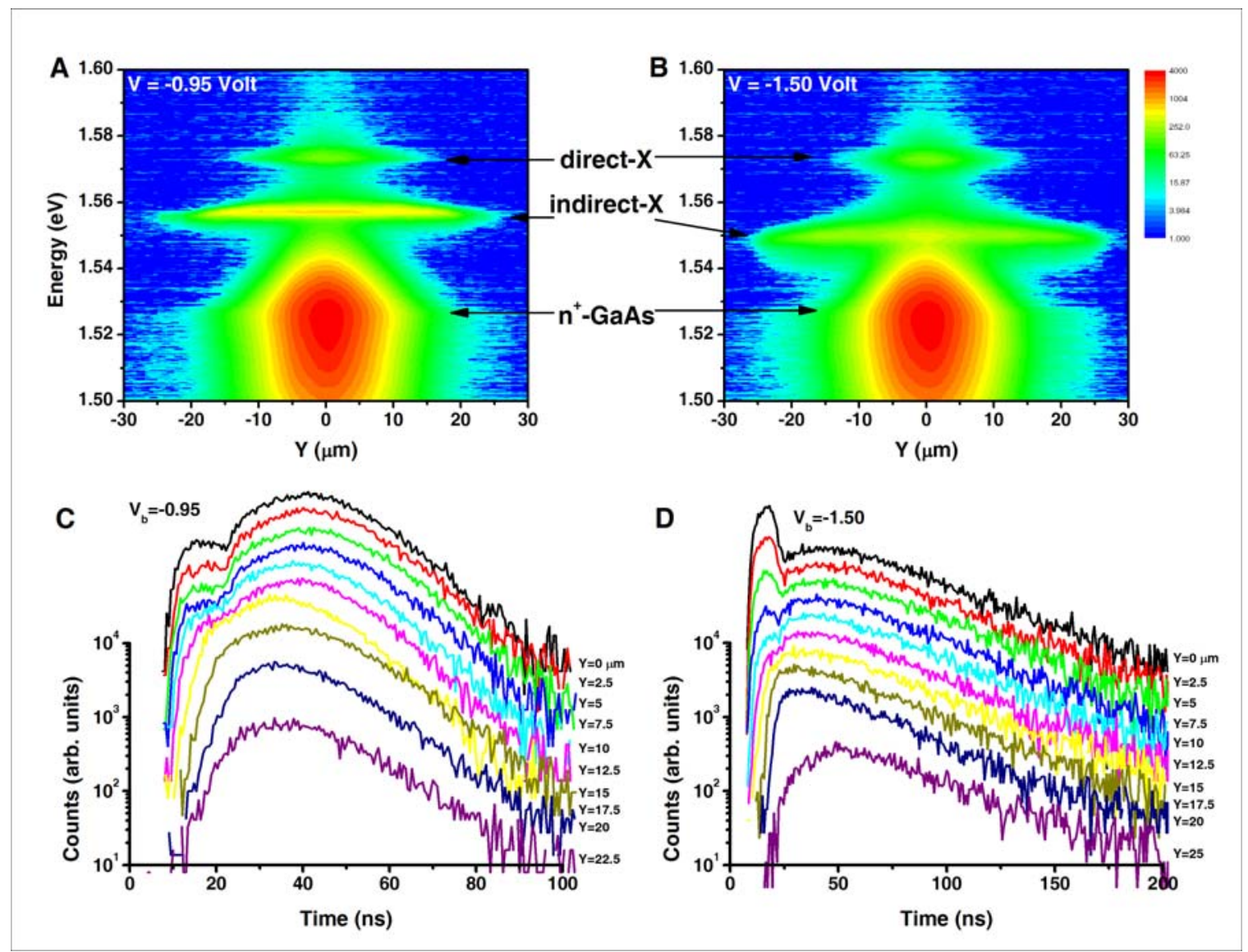

Figure 4.5 Spatially-resolved time-integrated PL spectra and time-resolved indirect-X PL at $V_{b}=-0.95$ and $-1.5 \mathrm{~V}$. (Laser diode excitation: spot radius $\sigma_{\mathrm{r}} \approx 7.5 \mu \mathrm{m}, \mathrm{h} \nu=1.944 \mathrm{eV}$, repetition rate $=1 \mathrm{MHz}$, pulse duration $\sim 20 \mathrm{ns,}$ and average $P_{\text {laser }} \approx 8.0 \mu \mathrm{W}\left[I_{\text {laser }} \approx 2.2 \mathrm{~W} / \mathrm{cm}^{-2}\right]$.)

After the generation of e-h pairs or formation of Xs, the carriers or Xs relax toward the ground state in momentum space while diffusing in real space due to the gradient in $\mathrm{X}$ density. This is characterized by the diffusion length $L_{D} \approx \sqrt{D \tau}$, D being the diffusion coefficient and $\tau$ is the carrier lifetime. Because indirect-Xs have a long lifetime, they travel further away from the generation center than the direct-Xs in QWs or carriers in the substrate $\mathrm{n}^{+}-\mathrm{GaAs}$ layers. This can be seen from the spatially-resolved time-integrated PL spectra (Figure 4.5A-B). The weak peak at $\mathrm{E} \approx 1.5673 \mathrm{eV}$ is the (intrawell) direct-X transition, and the low energy broad emissions are recombination of 
carriers in $\mathrm{n}^{+-G a A s}$ layers. Between these two is the (inter-well) indirect- $X$ transition, which shifts with the bias $V_{b}$ and persists to larger distances for higher $V_{b}$ 's. Because of the short lifetime $(<300 \mathrm{ps})$ and negligible diffusion of photogenerated carriers in $\mathrm{n}^{+}-$ GaAs, $\mathrm{n}^{+}$-PL can be used to measure the laser spot size and calibrate the time zero of the photon counting system. The laser spot radius was $\sigma_{\mathrm{r}} \approx 7.5 \mu \mathrm{m}$, which was used to determine the excitation intensity by $I_{\text {laser }}=P_{\text {laser }} /\left(2 \pi \sigma^{2}\right)$.

The time-integrated PL spectra were measured by a CCD camera attached to the spectrometer (see Figure 3.4 for experimental setup). The dispersed PL spectra can be redirected into a micro-channel photomultiplier attached to a second exist slit for TRPL measurements, where indirect-X PL peaks were centered at the exist slit with a bandwidth $\Delta \mathrm{E} \approx 1.7 \mathrm{meV}$. Spatially-resolved TRPL were measured by scanning a pinhole (equivalent to a diameter $<3 \mu \mathrm{m}$ on the sample) across the excitation spot in the confocal image plane. Near the center of the excitation spot where photocarrier generation and recombination occur both in the CQW and $\mathrm{n}^{+}$-GaAs layers, overlap of indirect-X PL and $\mathrm{n}^{+}$-PL through the exit slit of the spectrometer are inevitable (i.e. detected PL includes both indirect-X PL and $\mathrm{n}^{+}$-PL scattered through the exit slit). This overlap is more pronounced than that from direct-X PL under a higher $V_{b}$ due to the red shift of the indirect-X energy toward the $\mathrm{n}^{+}-\mathrm{PL}$ (Figure 4.5C-D). Carriers in the $\mathrm{n}^{+}$-GaAs layers have a short lifetime and diffusion length, therefore, $\mathrm{n}^{+}$-PL can be separated from indirect-X PL by temporal or spatial separation. The TRPL curves (or PL dynamics) in Figure 4.5CD show that the scattered $n^{+}-\mathrm{PL}$ decreases while the indirect-X PL decay time is almost constant with increasing $\mathrm{Y}$, the distance between the collection spot and the excitation center. The scattered $\mathrm{n}^{+}-\mathrm{PL}$ is negligible at a distance $\mathrm{Y}>10 \mu \mathrm{m}$ (spatial separation) or 
after switching off the excitation (temporal separation). The time resolution of the PL dynamics during and just subsequent to the laser excitation is limited by the diode laser intensity decay time, typically $\sim 300$ ps. The PL increase after the generation of e-h pairs can provide information on the $\mathrm{X}$ formation and relaxation processes. However, caution should be taken in interpreting the data when using a laser diode as the excitation source. Butov et al. have used the non-linear PL dynamics measured under laser-diode excitations as evidence for stimulated scattering of Xs $[37,133,134]$. Those results are questionable because indirect-X PL signals were not properly separated (spatially or temporally) from $\mathrm{n}^{+}$-PL signals.

\subsubsection{Electric field dependence}

In this section the TRPL of the spatially indirect-Xs measured as a function the bias voltage $V_{b}$ (or the electric field) are described. Because the spatially-resolved TRPL measurements have an almost constant decay time across the excitation spot, the pinhole was removed for $V_{b}$-dependent TRPL, which allows spatial averaging and a dramatic increase in the photon count. However, as a precaution, since the ratio of the $\mathrm{n}^{+}-\mathrm{PL}$ to indirect-X PL increases with $P_{\text {laser }}$ in this range (Figure 4.10), the average $P_{\text {laser }}$ was lowered to $\sim 4.2 \mu \mathrm{W}$ to reduce the fraction of the scattered $\mathrm{n}+$-PL signals detected at the indirect-X wavelength. The increased $\mathrm{n}^{+}-\mathrm{PL}$ has no impact on the determination of indirect-X PL decay time since $\mathrm{n}^{+}$-PL is negligible after switching-off of the laser excitation pulse. However, it could confuse the interpretation during excitation. 

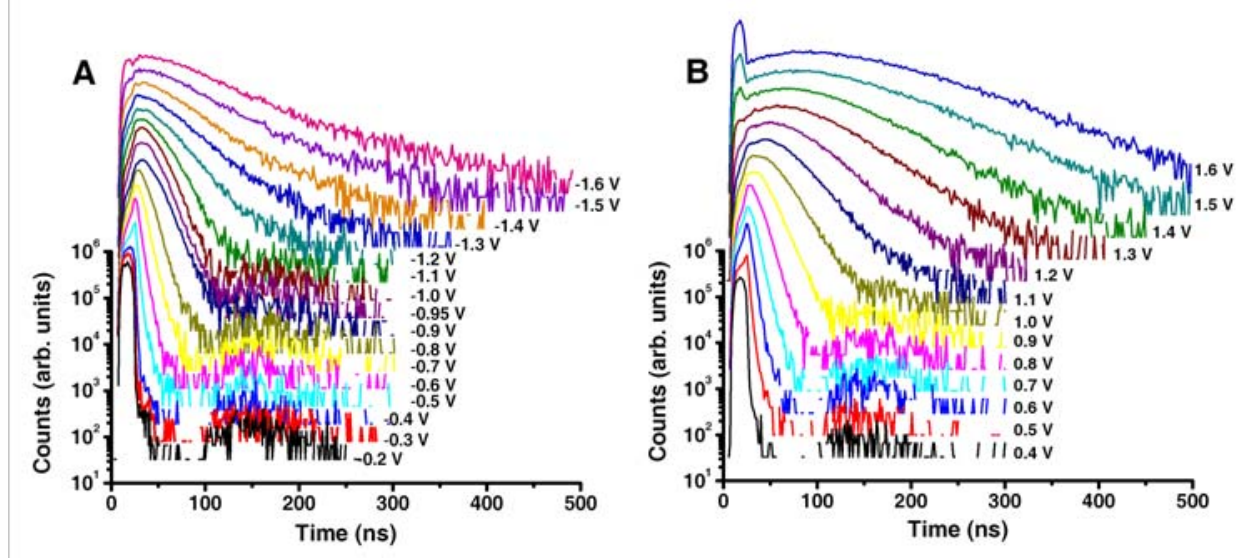

Figure 4.6 $V_{b}$-dependent TRPL indirect-Xs: (A) Negative and (B) positive bias voltages. The PL spikes in the square pulse excitation duration $(\sim 20$ ns) are mainly $\mathrm{n}^{+}-\mathrm{PL}$. (Laser diode excitation: spot radius $\sigma_{\mathrm{r}} \approx 7.5 \mu \mathrm{m}$, hv $=1.944 \mathrm{eV}$, repetition rate $=1 \mathrm{MHz}$, pulse duration $\sim 20 \mathrm{~ns}$, and average $\left.P_{\text {laser }} \approx 4.2 \mu \mathrm{W}\left[I_{\text {laser }} \approx 1.2 \mathrm{~W} / \mathrm{cm}^{-2}\right]\right)$.

In Figure 4.6A-B the TRPL of direct-Xs or indirect-Xs measured as a function of $V_{b}$ are shown for both positive and negative bias voltages. The $V_{b}$-dependent decay times ( $\tau$ ) obtained by an exponential fit are summarized in Figure 4.7. With resprect to the direct-X PL decay time ( $\sim 300 \mathrm{ps})$, the indirect-X PL decay time is increased by more than two orders of magnitude to $\sim 60 \mathrm{~ns}$ for a bias field $\mathrm{F} \approx 35 \mathrm{kV} / \mathrm{cm}$. As mentioned previously, $\tau$ is also affected by localization and the presence of free carriers associated with leakage current or trapping. Alsot evident from these plots is an asymmetry with respect to positive and negative bias. This is reflected in the photocurrent-voltage (I-V) characteristics of the sample in the presence of excitation in the AlGaAs barriers. Moreover, there is significant current flow for $V_{b}<0$ and a negative differential resistance $(\mathrm{NDR})$ regime for $V_{b} \approx-1.0 \mathrm{~V}$ (see Sec. 7.2.2). These I-V characteristics are evidence of the presence of charge or free carriers in the CQW structure, which can also result in the asymmetric $\tau_{\mathrm{X}}$ for positive and negative $V_{b}$ 's as seen in Figure 4.7. Note that 
the kink of $\tau_{\mathrm{X}}\left(V_{b}\right)$ near $-1.1 \mathrm{~V}$ coincides with the NDR regime, which suggest strong effects due to space charges or free carriers.
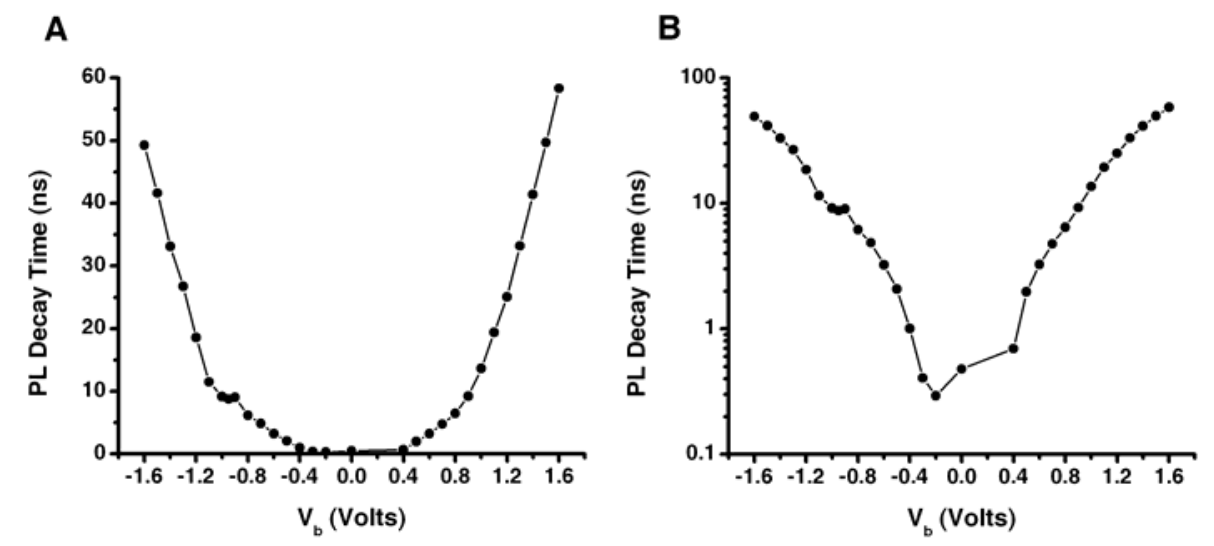

Figure 4.7 Indirect-X PL decay time versus $\mathrm{V}_{\mathrm{b}}$ : (A) linear-liner scale and (B) linear-log scale.

\subsubsection{Excitation intensity dependence}

Measuring TRPL of indirect-Xs as a function of $P_{\text {laser, which is related to }}$ photogenerated $\mathrm{X}$ density, is necessary for the following two reasons: (1) Indirect-X densities can affect $\mathrm{X}$ lifetimes and relaxation processes due to (a) X-X scattering or $\mathrm{X}$ free carrier scattering [135,136] for high densities, and (b) localization of Xs $[137,138]$ originated from interface disorders for low densities. (2) Assuming the $\mathrm{X}$ transition is a two level closed system with negligible nonradiative recombination, the steady state number of Xs $\left(\mathrm{N}_{\mathrm{X}}\right)$ can be approximated by $\mathrm{N}_{X} \approx \tau_{X} \times \mathrm{N}_{\mathrm{ph}}$, where $\mathrm{N}_{\mathrm{ph}}$ is the emitted $\mathrm{X}$ photon per unit time and $\tau_{X}$ is the effective $X$ lifetime. Because $\tau_{X}$ can depend on $N_{X}$ or $P_{\text {laser }}$, measuring $\tau_{\mathrm{X}}$ versus excitation intensity will thus provide a more accurate extimation of the $\mathrm{X}$ density. 

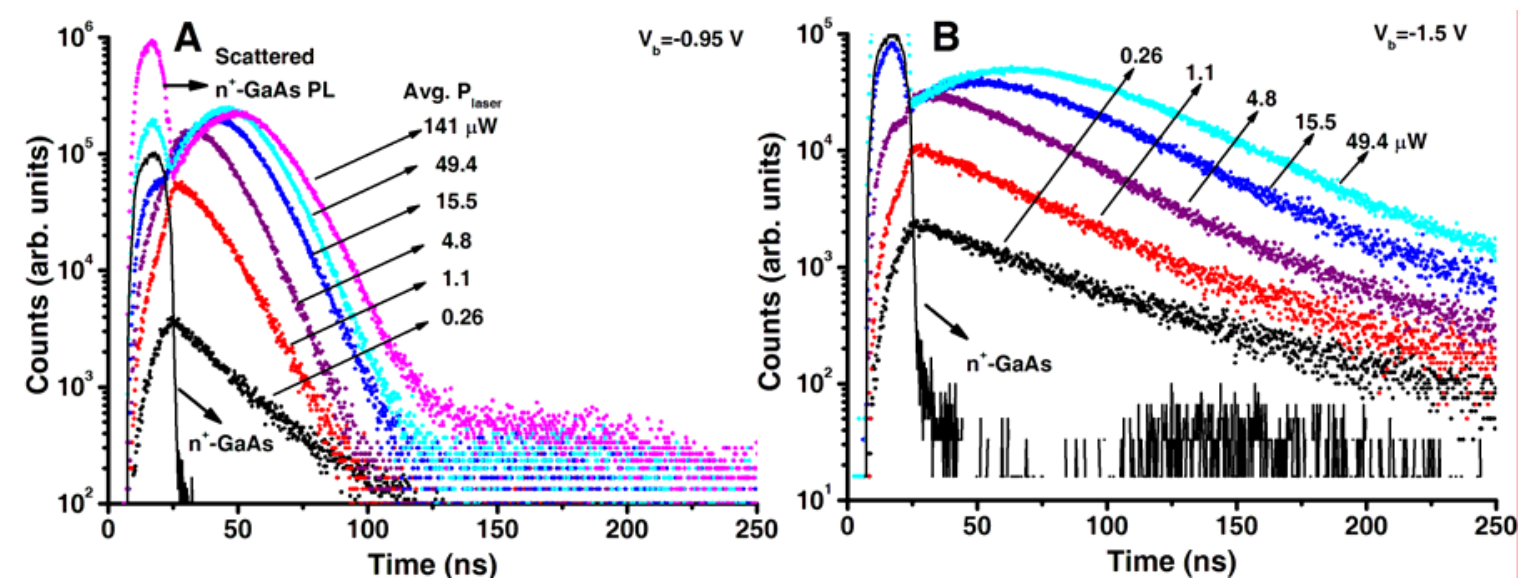

Figure 4.8 Laser excitation intensity ( $\mathrm{P}_{\text {laser }}$ ) dependent PL dynamics. (Laser diode excitation: spot radius $\sigma_{\mathrm{r}} \approx 7.5 \mu \mathrm{m}, \mathrm{h} v=1.944 \mathrm{eV}$, repetition rate $=1 \mathrm{MHz}$, pulse duration $\sim 20 \mathrm{~ns}$ ).

Figure 4.8 shows the TRPL of indirect-Xs and $\mathrm{n}^{+}-\mathrm{PL}$. The $\mathrm{n}^{+}$-PL exhibit a fast decay time $\sim 200 \mathrm{ps}$, which is likely to be limited by the decay time of the pulsed diode laser excitation. We use the fast $\mathrm{n}^{+}$-PL rise and decay to determine the system time-zero.

TRPL of indirect-Xs at $V_{b}=-0.95 \mathrm{~V}$ and $-1.5 \mathrm{~V}$ exhibits simple exponential decay at low $P_{\text {laser, }}$, but non-exponential decay at high $P_{\text {laser }}$. Therefore, we determine the PL decay times by two methods: (i) an exponential fit ( $\tau$, decay time) and (ii) the time for the PL intensity to fall to $1 / \mathrm{e}$ of its peak ( $\tau_{\mathrm{X}}$, fall time). Figure $4.9 \mathrm{~A}$ shows the determined indirect-X PL decay and fall times for $V_{b}=-0.95 \mathrm{~V}$ and $-1.5 \mathrm{~V}$. The indirect-X PL decay time levels off to almost a constant over a wide range of moderate $P_{\text {laser }}$. In this regime, indirect-X lifetimes are mainly determined by overlap of the wavefunctions of the constituent electron and hole, which is almost independent of indirect-X density. However, X PL decay times increase both at low density and hight density. At low density, Xs are localized due to in-plane disorder; whereas at high density, scattering between Xs and free carriers become important. Both effects can increase PL decay times or indirect-X lifetimes. X PL decay times increase for $I_{\text {laser }}>3 \mathrm{~W} / \mathrm{cm}^{2}\left(\equiv \mathrm{I}_{\mathrm{c} 2}\right)$, where a 
Mott transition is likely to occur and electron-hole plasmas are formed. We also determined the peak rise time after the excitation was switched off. ${ }^{16}$ The rise times increase dramatically at $I_{\text {laser }} \approx 0.6 \mathrm{~W} / \mathrm{cm}^{-2}\left(\equiv \mathrm{I}_{\mathrm{cl}}\right)$, where peak indirect-X intensities start deviating from a linear growth trend as well. Under the same $\mathrm{HeNe} \mathrm{CW}$ laser excitation intensity, peak indirect-X intensities also deviate from a linear growth with increasing $P_{\text {laser }}$ (Figure 4.3). For the same average $P_{\text {laser }}$ 's, the pulsed diode laser excitation has a $\sim 50$ times higher instant peak excitation intensity ( $2 \%$ duty cycle) than a CW HeNe laser. However, the time-integrated PL spectra under the pulsed excitation exhibits similar

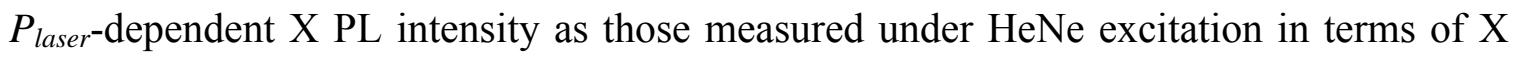
PL energy, line shape, and intensity (Figure 4.3, Figure 4.9, and Figure 4.10). Therefore, the PL spectra depend on average excitation intensities rather than on instantaneous intensities.
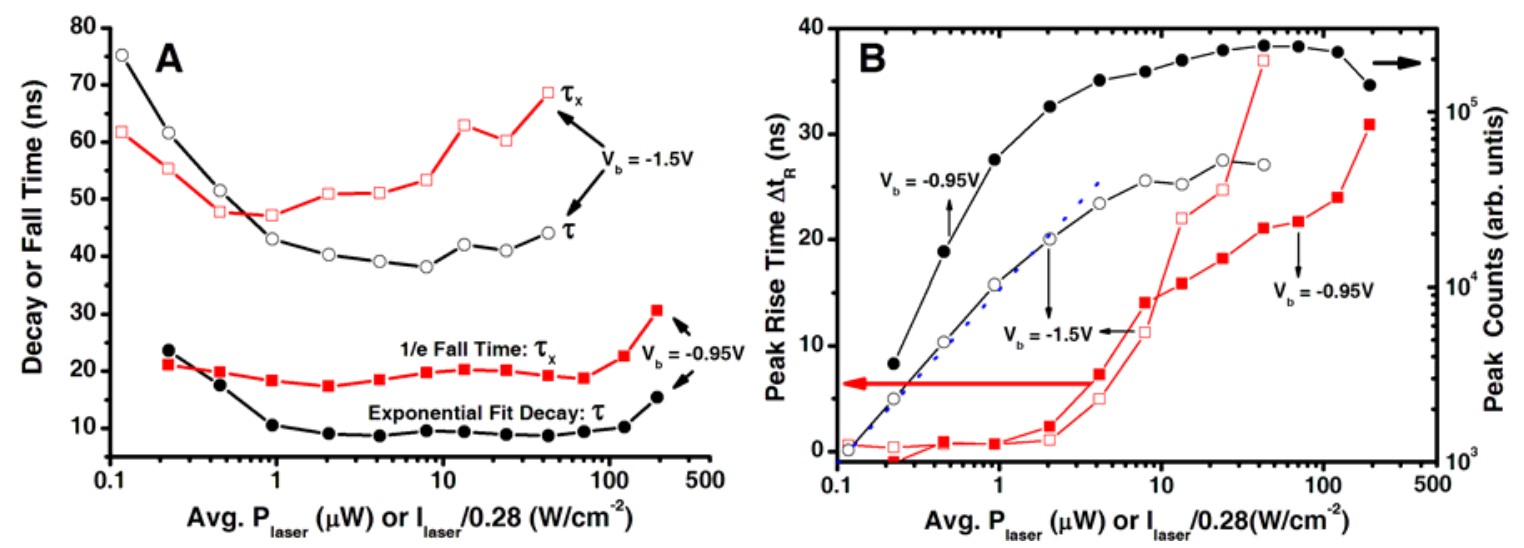

Figure 4.9 $P_{\text {laser }}$-dependent X PL decay/rise time under $V_{b}=-0.95 \mathrm{~V}$ and $1.5 \mathrm{~V}$. (Laser excitation: 1-MHz repetition rate, 20-ns pulse duration: $2 \%$ duty cycle).

\footnotetext{
${ }^{16}$ The pulse is considered switched off when $\mathrm{n}^{+}-\mathrm{PL}$ dropped to $1 \%$ of its peak intensity.
} 


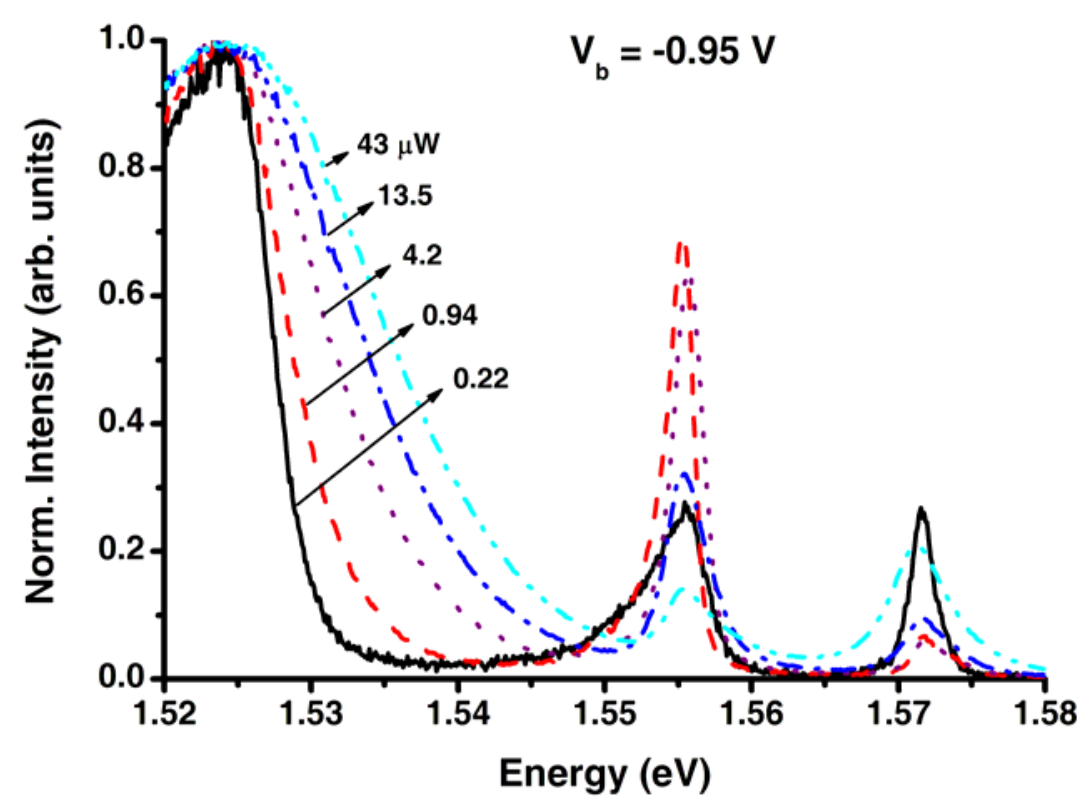

Figure 4.10 Normalized time-integrated PL spectra versus $P_{\text {laser }}$ at $V_{b}=$ $0.95 \mathrm{~V}$ under the pulsed laser diode excitation (1-MHz repetition rate, 20ns pulse duration: $2 \%$ duty cycle). Indirect-X peaks become more intense compared to $\mathrm{n}^{+}-\mathrm{PL}$ with decreasing $P_{\text {laser }}$ before $\mathrm{X}$ localization dominates at the low $P_{\text {laser }}$ regime.

Considering the $P_{\text {laser }}$-dependent time-resolved and time-integrated PL measurements, we tentatively attribute the two critical intensities $I_{c 1}$ and $I_{c 2}$ respectively to onset of (i) Fermion statistics of the constituent electrons and holes and (ii) Mott transition (significant $\mathrm{X}$ ionization or e-h plasma formation). The estimated $\mathrm{X}$ densities are $\sim 10^{10} \mathrm{~cm}^{-2}$ for $\mathrm{I}_{\mathrm{c} 1}$ and $\sim 10^{11} \mathrm{~cm}^{-2}$ for $\mathrm{I}_{\mathrm{c} 2}$, respectively (see Sec. 4.5 for density estimation). The $\mathrm{X}$ can thus only be considered as non-interacting bosons up to a density well below the Mott density as suggested in ref. $[23,139]$. Note that the lower density limit for the bosonic behavior of Xs does not mean that Xs cannot undergo Bose-Einstein condensation (BEC) at higher densities. The results signify that the critical density for BEC cannot be taken as that for non-interacting bosons. Though Xs exist according to the Mott criterion, Fermi statistics and coulomb interactions due to the constituent electrons and holes must be taken into account. 


\subsubsection{Excitation pulse duration dependence}

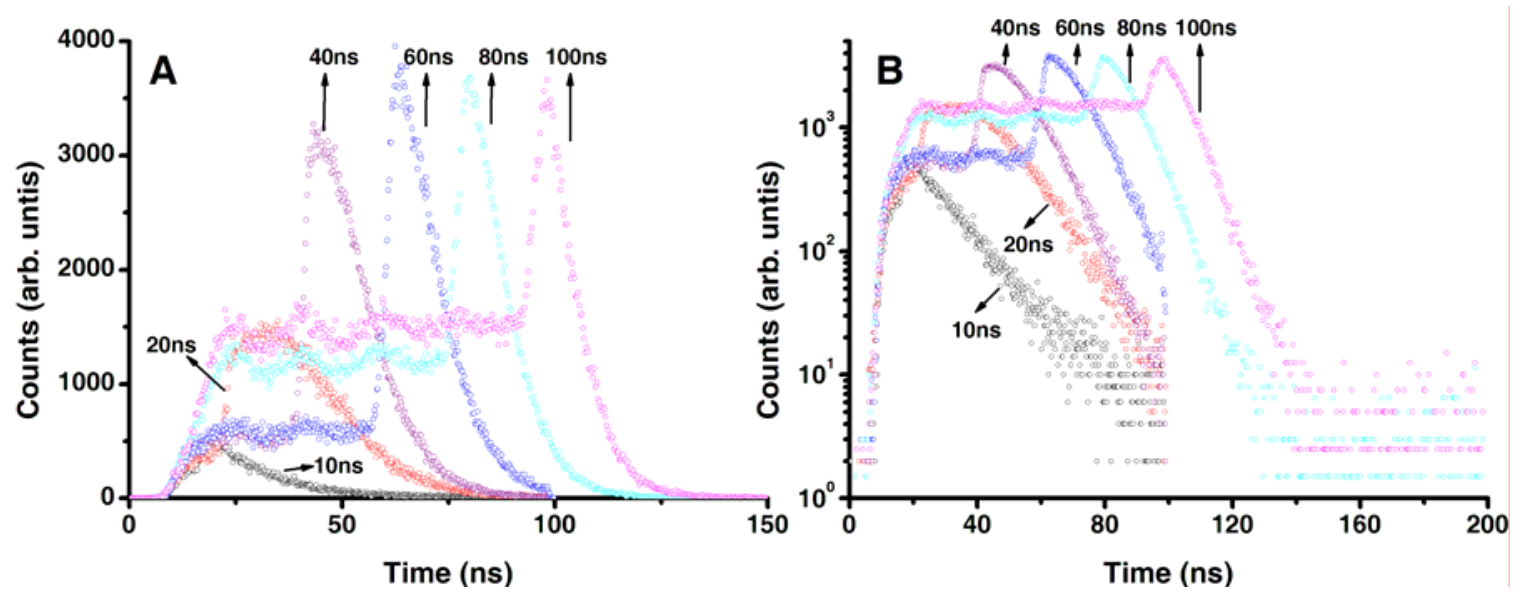

Figure 4.11 TRPL versus excitation pulse duration. (Laser diode excitation: spot radius $\sigma_{\mathrm{r}} \approx 7.5 \mu \mathrm{m}, \mathrm{h} v=1.944 \mathrm{eV}$, repetition rate $=1 \mathrm{MHz}$, and average $P_{\text {laser }} \approx 4.2 \mu \mathrm{W}\left[I_{\text {laser }} \approx 1.2 \mathrm{~W} / \mathrm{cm}^{-2}\right]$ ).

Thus far we have been concerned with TRPL measurements under laser diode excitation with 1-MHz repetition rate and 20-ns pulse duration. To understand the heating effects of photo-excitation and cooling processes of photocarriers or Xs, we also conducted TRPL with varying pulse durations.

Only $\mathrm{X}$ states with center-of-mass momenta $\mathrm{K} \approx 0$ are optically active and contribute to the X PL emissions (see Sec. 2.5). Thus TRPL of indirect-Xs represents the time evolution of the low energy occupation of optically active Xs. Under an above gap off-resonant excitation, PL is quenched during laser excitation due to a high effective temperature and thus the small fraction of cold low energy Xs. Figure 4.11 shows TRPL for pulse durations from $\sim 10 \mathrm{~ns}$ to $100 \mathrm{~ns}$. The indirect-X PL is quenched duration the pulse excitation, but rises sharply after the excitation pulse is switched off. The sharp rise ( 1-2ns) of the X PL after the excitation is switched off indicates a corresponding fast increase in occupation of optically active Xs. This signals a fast formation and thermal relaxation of indirect-Xs toward the quasi-equilibrium. 


\subsubsection{Temperature dependence}

The effects of temperature or thermalization on the radiative lifetime of Xs have been extensively studied $[99,140-142,67,143,144,138]$. The thermal equilibrium distribution of $\mathrm{X}$ states with different in-plane momenta produces an average decay time $(\tau)$ of the $\mathrm{X}$ population given by:

$$
\left\langle\tau^{-1}(T)\right\rangle=\frac{\int d E \rho(E) e^{-E / k_{B} T}}{\int d E \tau(E) \rho(E) e^{-E / k_{B} T}},
$$

where $\rho(E)$ is the excitonic density of states (a step function for a parabolic and isotropic band) [142]. Because the fraction of optically active Xs decreases when the temperature $(T)$ is raised, the lifetime is expected to increase with $T$ provided $\tau(\mathrm{E})$ is dominated by the radiative lifetime or thermalization It has been found theoretically and experimentally that $\tau(T) \propto T$ for Xs in quantum wells.
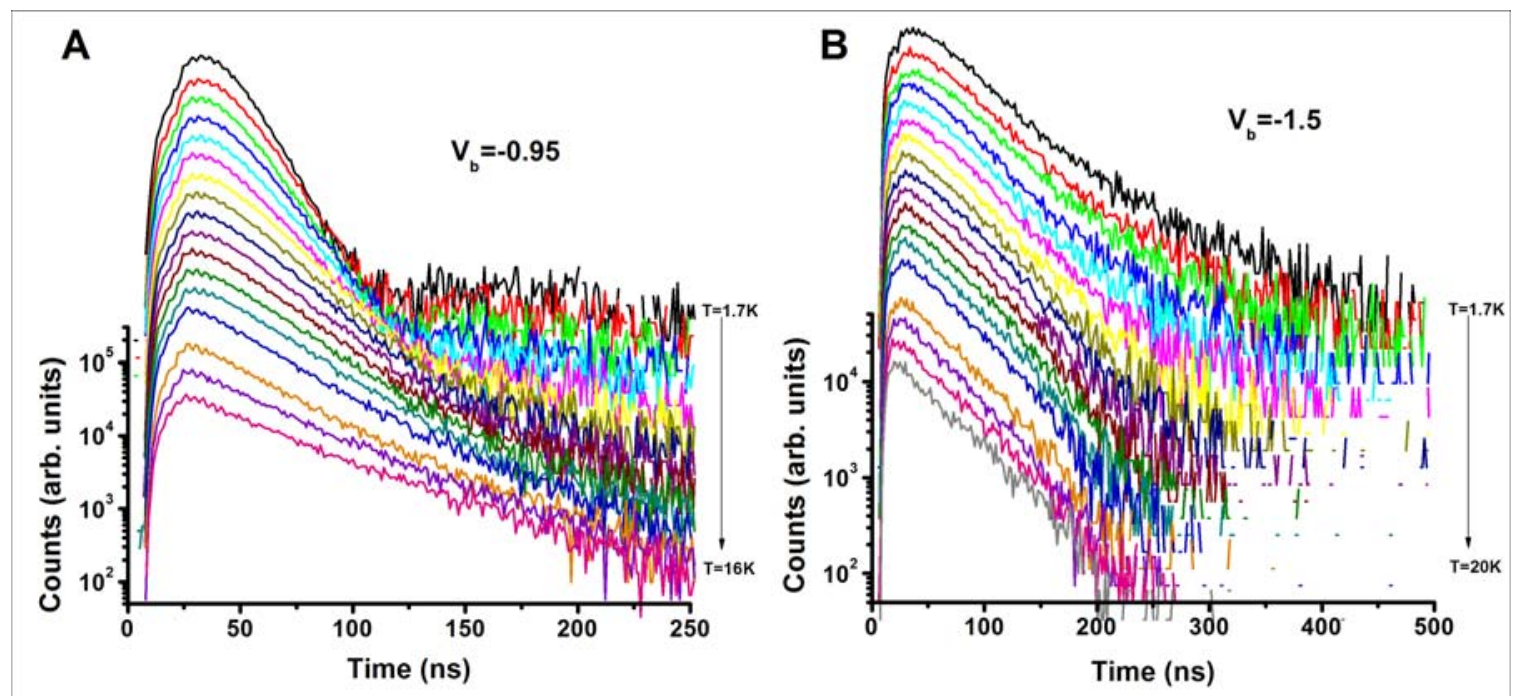

Figure 4.12 T-dependent indirect- $X$ TRPL at $V_{b}=-0.95 \mathrm{~V}$ and $-1.5 \mathrm{~V}$. (Laser diode excitation: spot radius $\sigma_{\mathrm{r}} \approx 7.5 \mu \mathrm{m}, \mathrm{h} v=1.944 \mathrm{eV}$, repetition rate $=1 \mathrm{MHz}$, pulse duration $\sim 20 \mathrm{~ns}$, and average $P_{\text {laser }} \approx 4.2 \mu \mathrm{W}\left[I_{\text {laser }} \approx\right.$ $\left.\left.1.2 \mathrm{~W} / \mathrm{cm}^{-2}\right]\right)$.

The $T$-dependent TRPL of indirect-Xs were measured at $V_{b}=-0.95 \mathrm{~V}$ and $-1.5 \mathrm{~V}$ from $T=1.7 \mathrm{~K}$ up to $\sim 20 \mathrm{~K}$ (Figure 4.12). TRPL curves exhibit exponential decays 
especially for higher temperatures. The exponential-fit decay times are shown in Figure 4.13A. At $V_{b}=-0.9 \mathrm{~V}$, the decay time increases linearly with the bath temperature $T$, consistent with the results reported for Xs in quantum wells. However, at $V_{b}=-1.5 \mathrm{~V}, \tau$ decreases first to a minimum at $T \approx 8 \mathrm{~K}$ before increasing with $T$. In order to understand the origin of such an unusual $T$-dependence, we measured time-integrated PL spectra under the same excitation conditions and $T$ as well.
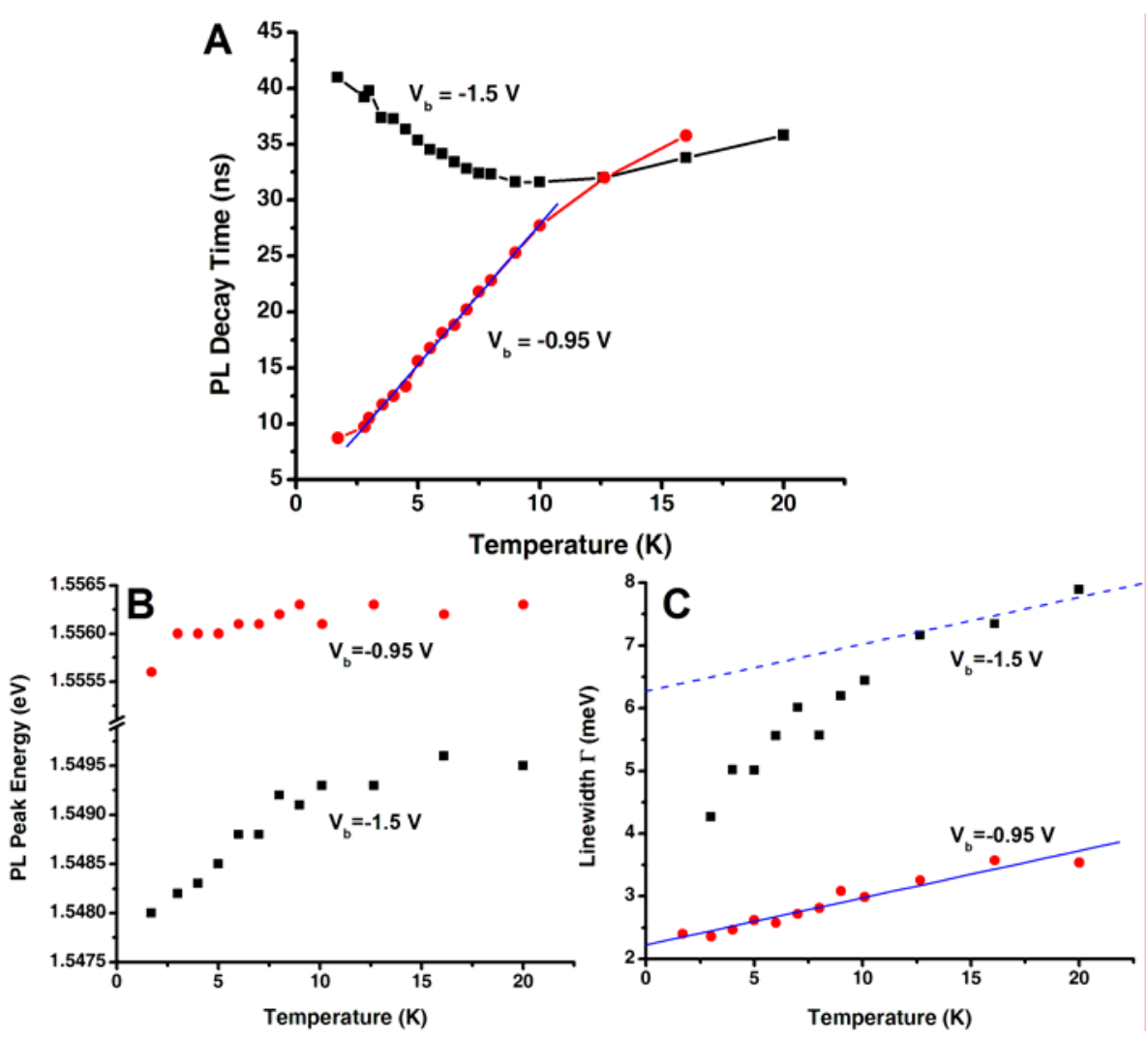

Figure $4.13 T$-dependent indirect-X PL decay time $(\tau)$ : (A) $\tau$ deduced from TRPL. At $V_{b}=-0.95 \mathrm{~V}, \tau$ increases linearly with $\mathrm{T}$ as $\tau$ (ns) $=13.83$ (ns) $0.053 \times T(\mathrm{~K})$. At $V_{b}=-1.5 \mathrm{~V}, \tau(T)$ decreases to a minimum at $T \approx 8 \mathrm{~K}$. (B) Indirect-X PL peak energy and (C) linewidth $\Gamma$ deduced from timeintegrated PL spectra. As $T$ increases, the PL peak energies blue shift and the linewidths broaden for both $V_{b}$ 's. (Laser diode excitation: spot radius $\sigma_{\mathrm{r}} \approx 7.5 \mu \mathrm{m}, \mathrm{h} v=1.944 \mathrm{eV}$, repetition rate $=1 \mathrm{MHz}$, pulse duration $\sim 20 \mathrm{ns,}$ and average $P_{\text {laser }} \approx 4.2 \mu \mathrm{W}\left[I_{\text {laser }} \approx 1.2 \mathrm{~W} / \mathrm{cm}^{-2}\right]$ ).

We first examine the linewidth $(\Gamma)$ of $\mathrm{X}$ PL, which is related to the radiative $\mathrm{X}$ lifetime $[145,99,146,147]$. At $V_{b}=-0.95 \mathrm{~V}, \Gamma$ increases linearly with $T$ as indicated by the 
linear fit (blue line) in Figure 4.13C, consistent with a linear linewidth broadening with increasing $T$ due to the interaction with LA phonons [145]. At $V_{b}=-0.95 \mathrm{~V}, \Gamma$ exhibits identical linear dependent slope for $T>10 \mathrm{~K}$ (blue dashed line) with a different low temperature dependence.

$\mathrm{X}$ energies also exhibit unusual $T$-dependent blue shifts (Figure 4.13C). (i) At $V_{b}$ $=-0.95 \mathrm{~V}$, average $\mathrm{X}$ densities $\left(\mathrm{N}_{\mathrm{X}}\right)$ increase with $T$ due to an increased lifetime if nonradiative recombination is negligible. Therefore, as $T$ is increased, a blue shift due to the mean-field dipole-dipole interaction (see 4.5.1) with increased $\mathrm{N}_{X}$ is possible. This blue shift is compensated by the thermal bandgap shrinkage ${ }^{17}(\sim 0.5-1 \mathrm{meV})[51,148]$ and is expected to be small (a few meV at most). The observed minor blue shifts $(\sim 0.5 \mathrm{meV})$ with increasing $\mathrm{T}$ can thus be induced by increased $\mathrm{X}$ densities due to the dipole-dipole interaction.

At $V_{b}=-1.5 \mathrm{~V}$, the blue shift is more significant $(>1.5 \mathrm{meV})$ and the PL decays faster with increasing $T$. Average $\mathrm{N}_{\mathrm{X}}$ will decrease with a shorter lifetime; therefore, the mean-field dipole-dipole interaction of indirect- $\mathrm{X}$ is unlikely to account for the large blue shifts. The photocurrent-voltage characteristics under such a pulsed laser diode excitation shows a significant photocurrent flow and gain at $V_{b}=-1.5 \mathrm{~V}$, which is similar to the I-V characteristics under a CW HeNe excitation (see Sec. 7.2.2). This signals a build-up of space charges or trapping of charge carriers, which can screen the external applied electric field and counter the Stark effect. This can thus affect the X lifetime and energy. Currently, we cannot determine the T-dependence of such a screening effect

\footnotetext{
17 An empirical equation for temperature dependent bandgap of $\operatorname{GaAs}(\Gamma$ valley): $E_{g}^{(\Gamma)}=1.519-5.405 \times 10^{-4} T^{2} /(T+204) e V$.
} 
quantitatively. Nevertheless, the significant blue shifts and reduction of PL decay times with $T$ at $V_{b}=-1.5 \mathrm{~V}$ is likely to be associated with the presence of space charges or cross-well carrier transport.

\subsubsection{Excitation energy dependence}

The electric field field dependent TRPL was also measured for a laser diode excitation of energy hv $=1.80 \mathrm{eV}$, which is below the band gap of the AlGaAs barriers $\left(\mathrm{E}_{\mathrm{g}}=1.84 \mathrm{eV}\right)$, and thus avoids generation of photocarriers in the AlGaAs layers. For the same $V_{b}$, TRPL of indirect-Xs (Figure 4.14A) excited by $\mathrm{h} v=1.80 \mathrm{eV}$ photons rises faster than that for excitation of $h v=1.944 \mathrm{eV}$, which signals a faster thermal relaxation rate. The PL decay curves are, however, qualitative the same (Figure 4.14B). More extensive TRPL measurements are required for $\mathrm{h} v=1.80 \mathrm{eV}$ as well as other excitation energies to understand the effects of the excitation energy.
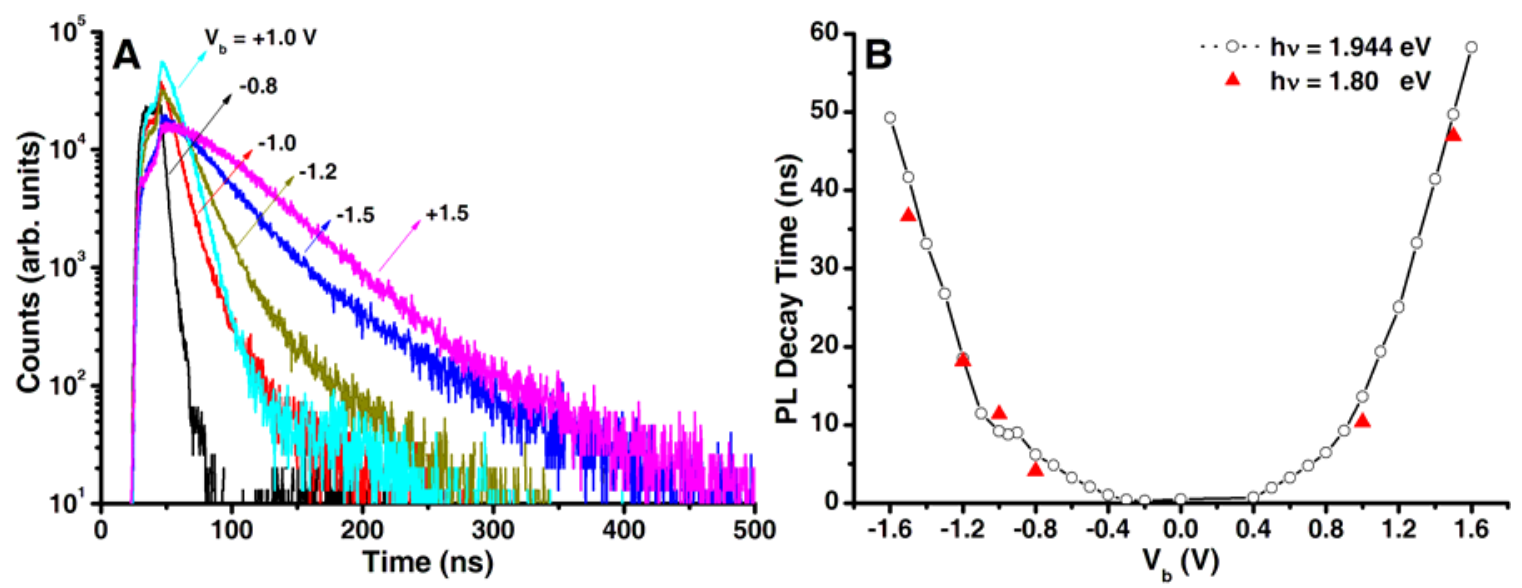

Figure 4.14 $V_{b}$-dependent TRPL indirect-Xs at an excitation energy $h v=$ $1.80 \mathrm{eV}$ : (A) Indirect-X TRPL for selected $V_{b}$ 's. (B) Indirect-X PL decay times: Figure 4.7A at $\mathrm{h} v=1.944 \mathrm{eV}$ is reproduced here for comparison. (Laser diode excitation: spot radius $\sigma_{\mathrm{r}} \approx 8 \mu \mathrm{m}, \mathrm{h} v=1.80 \mathrm{eV}$, repetition rate $=1 \mathrm{MHz}$, pulse duration $\sim 20 \mathrm{~ns}$, and average $P_{\text {laser }} \approx 8.5 \mu \mathrm{W}$ ). 


\subsection{Exciton density}

To estimate the density of Xs, we used two methods: (i) measurements of the PL peak blue shift (Sec. 4.5.1), and (ii) careful measurements and calibration of the photon collection efficiency of the PL imaging and detection system (Sec. 4.5.2).

\subsubsection{Mean field energy and exciton energy shift}

The single exciton Hamiltonian given in Sec. 4.2 does not include many-body interaction of Xs, which can have significant effects on the phases and states of an X system. Here we discuss only some essential condepts that are related to the $\mathrm{X}$ energy shift and density estimation.

There are several effects that can induce an energy shift of indirect-X PL [149]: (i) electric field (Stark effect), (ii) mean-field blue shift (due to the X-X interaction), (iii) thermal bandgap shrinkage, (iv) bandgap renormalization due to a high carrier density [150], (v) strain, and (vi) magnetic fields. The mean-field blue shift due to X-X interaction is the focus here.

In multiple quantum wells, a blue shift of the X PL line with increasing density was reported $[151,152]$ and attributed to a net X-X interaction [66]. For spatially indirect$\mathrm{Xs}$ in CQW, separation of the electron and hole in the z-direction gives rise to a dipole moment; therefore, the blue shift of X PL energy with X density is more pronounced due to dipole-diple interactions. This density dependent blue shift of indirect-X PL in GaAs/AlGaAs CQW systems has been reported $[153,154]$ and was also detailed in Sec. 4.3.2 under a HeNe laser excitation. In CQW systems, the effective $\mathrm{X}-\mathrm{X}$ interaction is dominated by the repulsive dipole-dipole interaction when the interspacing is large (i.e. low $\mathrm{X}$ density) [155-157]. The mean-field $\mathrm{X}$ energy can thus be approximated phenomenologically by 


$$
\begin{aligned}
E_{\text {mean-field }} & =\int_{0}^{2 \pi} d \theta \int_{r_{c}}^{\infty} r d r n \frac{(e d)^{2}}{4 \pi \varepsilon r^{3}} \\
& =\frac{n e^{2} d^{2}}{2 \varepsilon r_{c}}
\end{aligned}
$$

where $d$ is the e-h separation, $\varepsilon$ the dielectric constant, and $r_{c}=d / 2$ for a classical parallel plate capacitor [132]. This gives an $\mathrm{X}$ energy blue shift $\delta E \approx n e^{2} d / \varepsilon$, which allows estimation of the $\mathrm{X}$ density. For the CQW used, $d \approx 12 \mathrm{~nm}$ and $\varepsilon \approx 12.4 \varepsilon_{0}$ (low temperature static dielectric constant) [148], the density can thus be estimated by $n \approx 5.72 \times 10^{9}\left(\mathrm{meV}^{-1} \bullet \mathrm{cm}^{-2}\right) \times \delta E(\mathrm{meV})$. However, this phenomenological formula is less reliable at high densities, where the exchange terms between the electrons and holes of different Xs may be important $[19,110,158]$. The density dependent blue shift for indirect-Xs in the CQW structure studied here has been calculated with a mean-field approximation including the exchange correction for Xs by de-Leon et al. [110]. Assuming an equal concentration of Xs with different spins, the $\mathrm{X}$ density can then be approximated by $n \approx 8.0 \times 10^{9}\left(\mathrm{meV}^{-1} \cdot \mathrm{cm}^{-2}\right) \times \delta E(\mathrm{meV})$, which is higher but close to the density obtained from the above phenomenological approximation without an exchange correction.

We have shown the $\mathrm{X}$ PL peak energy $\left(\mathrm{E}_{0}\right)$ versus $P_{\text {laser }}$ in Figure 4.3A. Extrapolating the peak indirect-X energy measured under a low uniform excitation (Figure 4.4), we deduced $\mathrm{E}_{0}\left(P_{\text {laser }}=0\right) \approx 1.553 \mathrm{eV}$ as the baseline to determine the indirect-X energy shift $\delta E$ from $\mathrm{E}_{0}$ shown in Figure 4.3A. X densities $\left(\mathrm{n}_{\mathrm{X}}\right)$ estimated from the above mentioned formula are represented by the solid red circles in Figure 4.15. For $I_{\text {laser }}<0.8 \mathrm{~W} / \mathrm{cm}^{-2}, \delta E \propto I_{\text {laser }}^{0.8}$ (red line), close to the linear dependence predicted by the 
mean-field approximation. However, for higher excitation intensities, it increases approximately with a power-law $\delta E \propto P_{\text {laser }}^{0.30}$ (red dash line), not linearly.

The $\mathrm{X}$ density can also be approximated by $\mathrm{n}_{\mathrm{X}} \approx \tau_{\mathrm{X}} \times \mathrm{G}_{\mathrm{e}-\mathrm{h}}$ (solid black squares), assuming a simple two level system and neglecting the escape/capture of the photogenerated carriers or nonradiative recombination. The generation rate of e-h pairs $\left(\mathrm{G}_{\mathrm{e}-\mathrm{h}}\right)$ in the two 8-nm GaAs QWs is estimated from the absorption coefficient $\alpha \approx$ $3.9 \times 10^{4} \mathrm{~cm}^{-1}$ [159] assuming $100 \%$ internal quantum efficiency. At $V_{b}=-0.95 \mathrm{~V}$, the effective $\mathrm{X}$ lifetime $\tau_{\mathrm{X}}$ is assumed to be about $20 \mathrm{~ns}$, which is almost constant for most $P_{\text {laser }}$ (see Figure 4.9A). X densities estimated by this method are represented by the sold squares in Figure 4.15. Note that this estimation could be inaccurate because of the error in the estimated $\alpha$ and additional capture/escape of photocarriers.

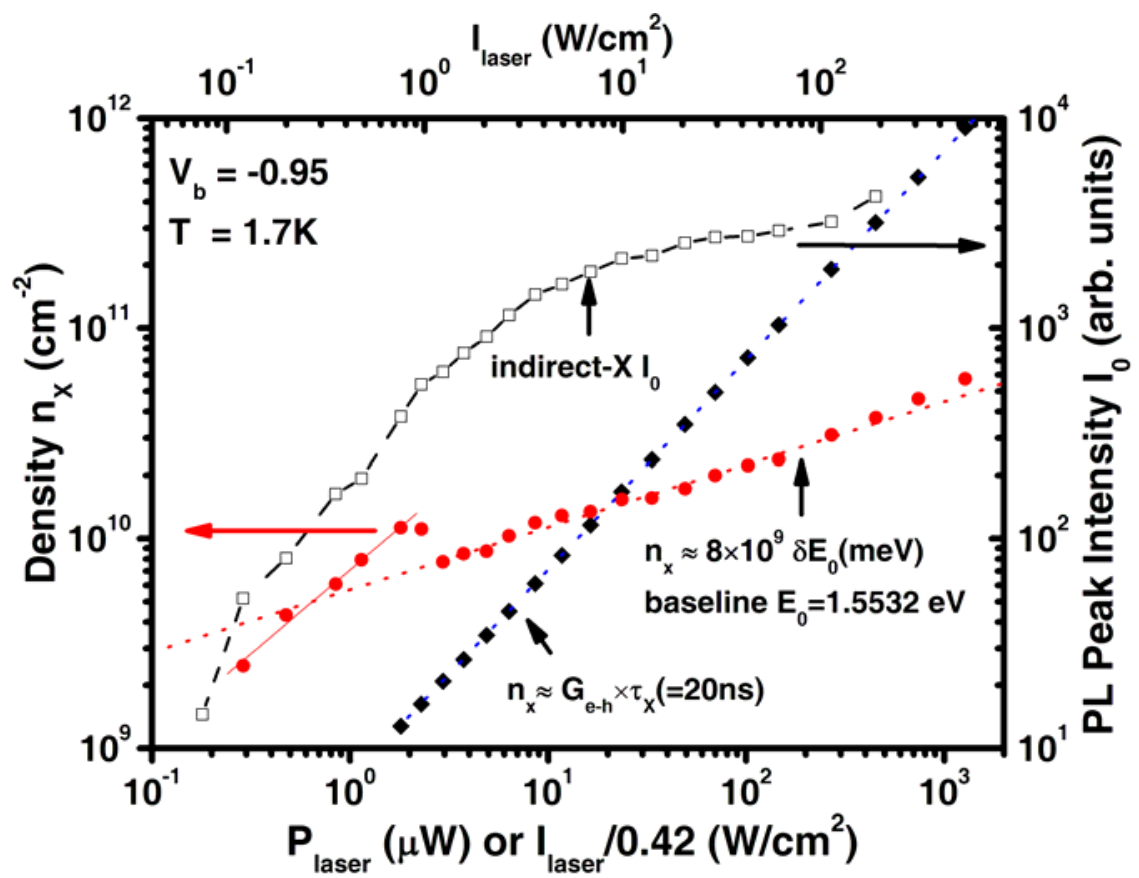

Figure 4.15 Estimated exciton density versus $P_{\text {laser }}$ under a bias $V_{b}=-0.95$ $V$. Red circles: densities estimated from $\delta \mathrm{E}$. Black squares: densities derived from e-h pairs generated in the two GaAs quantum wells. Black open squares: the peak X PL intensity $\left(\mathrm{I}_{0}\right)$. 
These two methods agree only for moderate $P_{\text {laser }}$. The discrepancy is likely caused by the presence of space charge or vertical carrier transport across and capture by the QWs. The HeNe laser excitation energy $(\mathrm{h} v=1.959 \mathrm{eV})$ is above the GaAs or AlGaAs bandgap $\left(\mathrm{E}_{\mathrm{g}}=1.519 \mathrm{eV}\right.$ for $\mathrm{GaAs}$ and $1.84 \mathrm{eV}$ for $\left.\mathrm{Al}_{0.33} \mathrm{Ga}_{0.67} \mathrm{As}\right)$, thus free carriers are generated all across the structure. These photogenerated free carriers can screen the dipolar indirect-X interactions, which leads to a reduction of X PL energy shift with X density. The high intensity excitation also could heat the lattice, which gives rise to a bandgap shrinkage (red shift) (see Sec. 4.4.5). Although weak, this red shift can cancel the blue shift caused by the X-X interaction.

\subsubsection{Photon collection efficiency}

Under the mean-field approximation, X PL energy blue shifts give reasonable X density estimation at low density regimes. However, the mean-field X energy shift is not reliable for high $\mathrm{X}$ densities due to (i) interactions other than dipole-dipole interaction among Xs, and (ii) non-ideal properties (e.g. the non-negligible photocurrent, see Sec. 7.2.2) for the CQW studied. We thus also estimated the $\mathrm{X}$ density with careful measurements and calibration of the photon collection efficiency of the PL imaging and detection system.

The photon collection efficiency $\left(\eta_{c}\right)$ of the optical imaging/detecting system can be determined by two independent methods:

(A). Careful measurements of the transmission of the optics and calculation of PL extraction efficiency $[160,161]$. We assume isotropic (i.e. no angular dependence) X PL emission from the CQW active region and use the given quantum efficiency of the detectors. 
(B). Use of a 'calibrated' photon source directly at the CQW active region. As discussed in Sec. 4.3, the capture and escape of carriers in and out of QWs are negligible with the absence of electric field in CQW. Therefore X PL emission under a zero bias (i.e. direct-X PL) can serve as a calibrated photon source, which is assumed to emit an X photon flux equal to the e-h pair generation rate $\left(\mathrm{G}_{\mathrm{e}-\mathrm{h}}\right)$ in the two 8-nm GaAs QWs. $\mathrm{G}_{\mathrm{e}-\mathrm{h}}$ can be determined as described in the previous section. The PL photon extraction efficiency of the optical system was then determined from the electronic signal $\left(S_{e}\right)$ by $\eta_{c}=S_{e} / G_{e-h}$.

The photon collection efficiencies determined by these two methods are consistent $\left(\eta_{\mathrm{c}}\right.$ differs less than $\left.20 \%\right)$. Once $\eta_{\mathrm{c}}$ is determined, the indirect-X photon flux $\left(\mathrm{N}_{\mathrm{X}, \mathrm{ph}}\right)$ under a bias voltage can be obtained by $N_{X}^{p h}=S_{e} / \eta_{c}$. The steady-state number of Xs $\left(\mathrm{N}_{\mathrm{X}}\right)$ can then be approximated by $N_{X}=\tau_{X} N_{X}^{p h}[162,161]$, where $\tau_{\mathrm{X}}$ is the effective $\mathrm{X}$ lifetime determined from TRPL measurements. Here $\mathrm{X}$ transitions are approximated as those in a two level closed system with a simple exponential decay and negligible nonradiative recombination. The $\mathrm{X}$ density can also be determined similarly from the $\mathrm{X}$ photon flux density for PL imaging measurements, which will be discussed in Chapter 5 .

\subsection{Effective mass and magneto-optical properties}

The exciton optical spectrum consists of discrete states - similar to the Rydberg states of hydrogen - each having a dispersion that reflects the movement of the exciton as a whole (Figure 2.2). In this section we show that the dispersion of indirect-Xs in CQW can be directly measured optically by applying an in-plane magnetic field $[163,164]$. This dispersion was used to determine the magnetoexciton effective mass which is critical to 
the observation of the collective exciton effects and becomes overwhelmingly larger than the sum of the electron and hole masses in high magnetic fields.

The magnetic fields also have many important effects on collective phenomena in the electron-hole (e-h) or exciton systems. For example single component 2D electron (or hole) gases exhibit the integer and fractional quantum Hall effects [87,165]. Two component 2D electron-hole neutral systems are also expected to possess curious properties at high magnetic fields. This is in particular the case of 2D-excitons in a perpendicular magnetic field (magnetoexcitons) whose composite e-h structure has been predicted to result in intriguing effects $[166,167,168]$ : (i) the 2D-magnetoexciton binding energy and effective mass are independent of the electron and hole effective masses and are determined by the magnetic field only [166]; (ii) although 2D-magnetoexciton is a neutral particle, it reacts to the application of in-plane electric field as a single charge (Hall effect for exciton) [167]; (iii) in a direct band gap semiconductor the 2Dmagnetoexciton ground state in in-plane electric field occurs at finite momentum $[167,169]$.

Here we focus on optically determining the indirect-X dispersion by applying a tilted magnetic field with a component parallel to the QW plane. The idea is to profice simultaneous breaking of time-reversal and space-inversion symmetry in the system. It can be understood by considering the usual momentum operator under a magnetic field, which commute with the Hamiltonian of excitons and now plays the role of the center-ofmass $(\mathrm{CM})$ momentum $[170,163]$ :

$$
P=M \vec{v}_{g}-\frac{e}{c} \vec{B} \times \vec{r}_{e h},
$$


where $\mathrm{M}$ is the exciton mass, $\vec{v}_{g}$ is the $\mathrm{CM}$ velocity, and $\vec{r}_{e h}=\vec{r}_{e}-\vec{r}_{h}$ is the coordinate of relative motion. The second term in Eq. (4.1) results from the Lorentz force acting on the electron and the hole that constitute the exciton. Only excitons with momentum $\mathrm{P} \sim 0$ can recombine radiatively; therefore, an exciton is optically active only when it has a velocity such that $M \vec{v}_{g}=\frac{e}{c} \vec{B} \times \vec{r}$. For indirect-X in CQW, the e-h distance $d$ is almost fixed by the potential barrier; if an in-plane magnetic field is applied, the indirect- $\mathrm{X}$ states shift rigidly in $\mathrm{k}$ space in the in-plane direction perpendicular to $\mathrm{B}$ [171]. This mechanism could also be used for obtaining a cold exciton gas in thermodynamic equilibrium. Because of the momentum shift, the in-plane magnetic field tunes the recombination lifetime of the indirect-Xs at rest to very high values, without affecting their binding energy [50].

Figure 4.16 illustrates the principle underlying the coupling mechanism for magnetoexcitons. Consider an in-plane free moving magnetoexciton whose CM momentum is $\mathrm{P}(\mathrm{x}, \mathrm{y})$ and dispersion law is $\mathrm{E}_{\mathrm{X}}=\mathrm{E}_{\mathrm{X}}(\mathrm{P})$. The magnetoexciton has a velocity, $\mathrm{v}_{\mathrm{g}}=\partial \mathrm{E}_{\mathrm{X}} / \partial \mathrm{P}$, and the attractive Coulomb force between the constituent electron and hole is cancelled exactly by the Lorentz force [166,167]. Applying this condition self-consistently, one can determine the magnetoexciton dispersion, the binding energy $E_{b}$ and, in turn, the effective mass $M_{B}$. In the high magnetic field limit, defined by the condition that $\mathrm{E}_{\mathrm{b}}$ is much smaller than the electron and hole cyclotron energies, the problem can be solved analytically [166]. Several conclusions are as follows: (i) $E_{B}=\sqrt{\pi / 2} e^{2} /\left(\varepsilon l_{B}\right) \sim \sqrt{B_{\perp}}$, where $B_{\perp}$ is the component of the magnetic field perpendicular to the QW plane, $l_{B}=\sqrt{\hbar c /\left(e B_{\perp}\right)}$ the magnetic length, and $\varepsilon$ the dielectric 
constant, (ii) $E_{X}(P)=-E_{B} e^{-\beta} I_{0}(\beta)$, where $\mathrm{I}_{0}(\beta)$ is the modified Bessel function, and $\beta=\left[P l_{B} /(2 \hbar)\right]^{2}, \quad$ (iii) for $\quad P l_{B} / \hbar \ll 1, M_{B}=\left(2^{3 / 2} \varepsilon \hbar^{2}\right) /\left(\pi^{1 / 2} e^{2} l_{B}\right) \sim \sqrt{B_{\perp}}, \quad$ (iv) for magnetoexciton with $\mathrm{P}=0$ the magnetic length plays the role of the Bohr radius, (v) magnetoexcitons with momentum P carry an electric dipole in the direction perpendicular to $\mathrm{P}$ whose magnitude, $\left\langle\vec{r}_{e h}\right\rangle=\hat{z} \times P l_{B}^{2} / \hbar$, is proportional to $\mathrm{P}$. This expression makes explicit the coupling between the center-of-mass motion and the internal structure. The coupling results in a curious property, called the electrostatic analogy: the dispersion $E_{X}(\mathrm{P})$ can be calculated from the expression of the Coulomb force between the electron and the hole as a function of $\left\langle\vec{r}_{e h}\right\rangle$ and has the consequence that the magnetoexciton mass depends on $\mathrm{B}_{\perp}$ only, independent of the electron and hole masses. In contrast to the e-h system at a zero magnetic field (hydrogen problem) all the e-h pairs are bound states and there is no scattering state. When $P l_{B} / \hbar \gg 1$, the separation between the electron and hole extends to infinity and the magnetoexciton energy evolves to the transition energy between the free electron and hole Landau levels. Figure 4.16B schematically compares magnetoexciton dispersions with and without the magnetic fields perpendicular to the QW plane. 

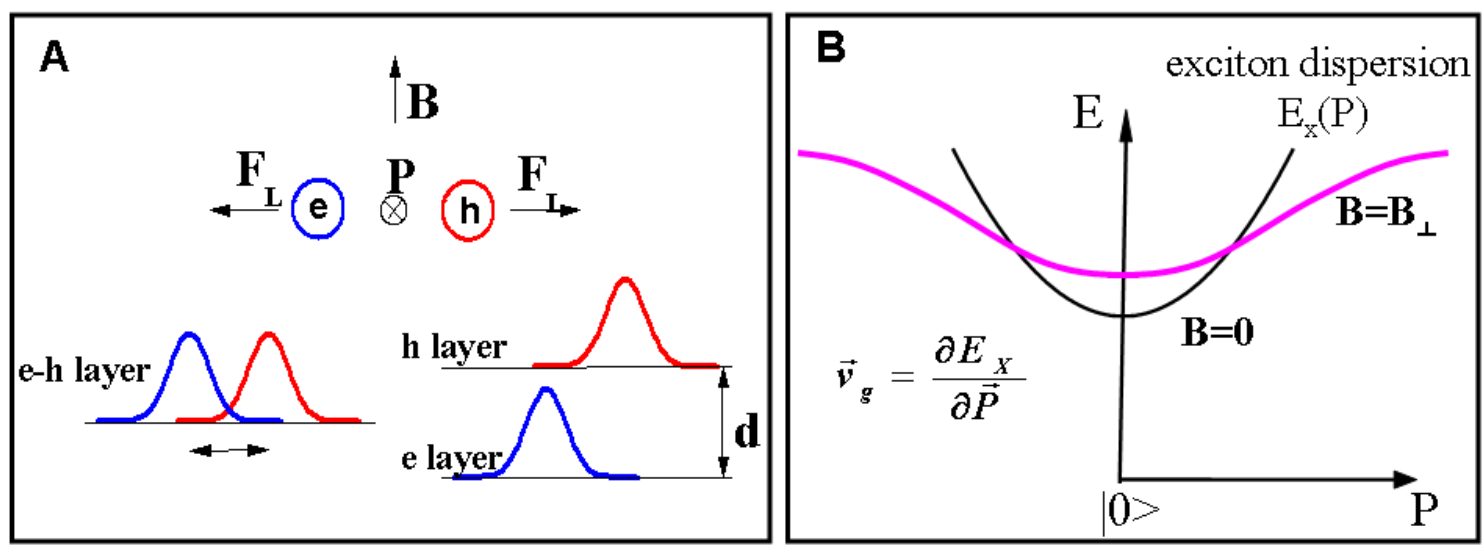

Figure 4.16 (A) Schematics showing (i) the coupling between the 2Dmagnetoexciton center-of-mass and internal motions (top), (ii) the separation between the electron and hole wave functions for quasi-2D magnetoexciton (bottom left) and quasi-2D indirect magnetoexciton (bottom right). $d$ is the mean e-h separation along $z$, the sample growth direction. (B) Example dispersions of 2D-magnetoexciton with and without the magnetic fields.

The principle of our experimental method is illustrated in Figure 4.17. The only free exciton states that can recombine radiatively are those with momentum near $\mathrm{P}_{0} \approx 0$, the intersection between the dispersion surface $\mathrm{E}_{\mathrm{X}}(\mathrm{P})$ and the photon cone $E_{p h}=P c / \sqrt{\varepsilon}$. In GaAs structures the radiative zone corresponds to small $\mathrm{CM}$ momenta $K \approx K_{0} \approx E_{g} \sqrt{\varepsilon} /(\hbar c) \approx 2.7 \times 10^{5} \mathrm{~cm}^{-1}$. Let us consider now a spatially indirect exciton with electron and hole confined in different layers parallel to the QW plane. Such an exciton possesses yet another interesting property. It has been shown in Ref. $[171,163]$ that when a magnetic field $\mathrm{B}_{\|}$is applied in the $\mathrm{QW}$ plane the exciton dispersion surface shifts by $P_{B}=-\frac{e}{c} d B_{\|}$(Figure 4.17). Therefore as $B_{\|}$increases the intersection of the photon cone with the exciton dispersion surface varies. Thus the indirect-X dispersion can be determined by measuring the magnetoexciton photoluminescence (PL) energy as a function of $\mathrm{B}_{\|}$. 
For the CQW studied, the shift induced by strong in-plane magnetic fields $K_{B}=\frac{e}{\hbar c} d B_{\|}$is much larger than $\mathrm{K}_{0}$. For example at $\mathrm{B}_{\|}=12 \mathrm{~T}, K_{B} \sim 2.1 \times 10^{6} \mathrm{~cm}^{-1} \sim 8 \times K_{0}$.

The peak energy of the indirect exciton PL is set by the energy of the radiative zone and is given as a function of in-plane magnetic field by $E_{P=0}=P_{B}^{2} / 2 M_{B}=e^{2} d^{2} B_{\|}^{2} /\left(2 M_{B} c^{2}\right)$ for a parabolic dispersion. The PL measurements were performed in tilted magnetic fields $\vec{B}=B_{\|} \hat{x}+B_{\perp} \hat{y}$ at $\mathrm{T}=1.8 \mathrm{~K}$ in a He cryostat with four optical windows. $\mathrm{B}_{\|}$was used to measure the dispersion of the magnetoexciton (Figure 4.17). Carriers were photoexcited by a HeNe laser and at an excitation intensity $\sim 0.1 \mathrm{~W} / \mathrm{cm}^{2}$.

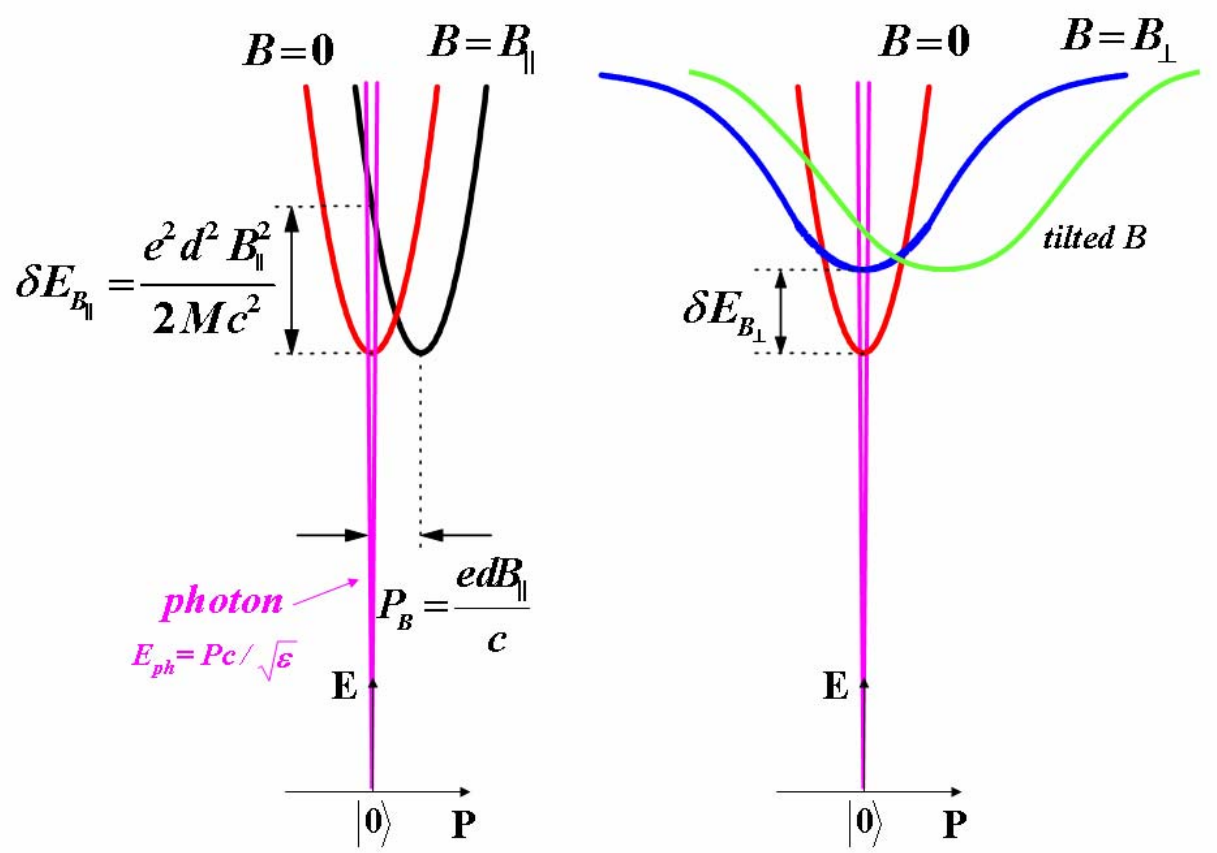

Figure 4.17 Schematics of the indirect exciton dispersions: (left) at zero and finite in-plane magnetic field $B_{\|}$, (right) at zero $B$, perpendicular $B_{\perp} d$ tilted $\mathrm{B}$. The optically active exciton states are near the radiative zone determined by the photon cone.

The basic features of the indirect magnetoexciton are the same as those of the direct magnetoexciton. They are also determined by the coupling between the 
magnetoexciton internal structure and the center-of-mass motion. However, because of the separation between the electron and hole layers the indirect magnetoexciton binding energy and effective mass differ quantitatively from those of direct magnetoexciton (Figure 4.16A). As d increases, the indirect magnetoexciton binding energy is reduced whereas its effective mass is increased. In particular, for $d \ll l_{B}, M_{B d}=M_{B}\left[1+2^{3 / 2} d /\left(\pi^{1 / 2} l_{B}\right)\right]$ and for $d \gg l_{B}, M_{B d}=M_{B} \pi^{1 / 2} d^{3} /\left(2^{3 / 2} l_{B}^{3}\right)[172]$. The effective mass enhancement is easily explained using the electrostatic analogy: For separated layers the e-h Coulomb interaction is weaker than that within a single layer and changes only slightly for $\vec{r}_{e h} \leq d$. This implies that $\mathrm{E}_{\mathrm{X}}(\mathrm{P})$ increases only slowly for $P \leq \hbar d / l_{B}{ }^{2}$; in other words, the indirect magnetoexcitons have a large effective mass.

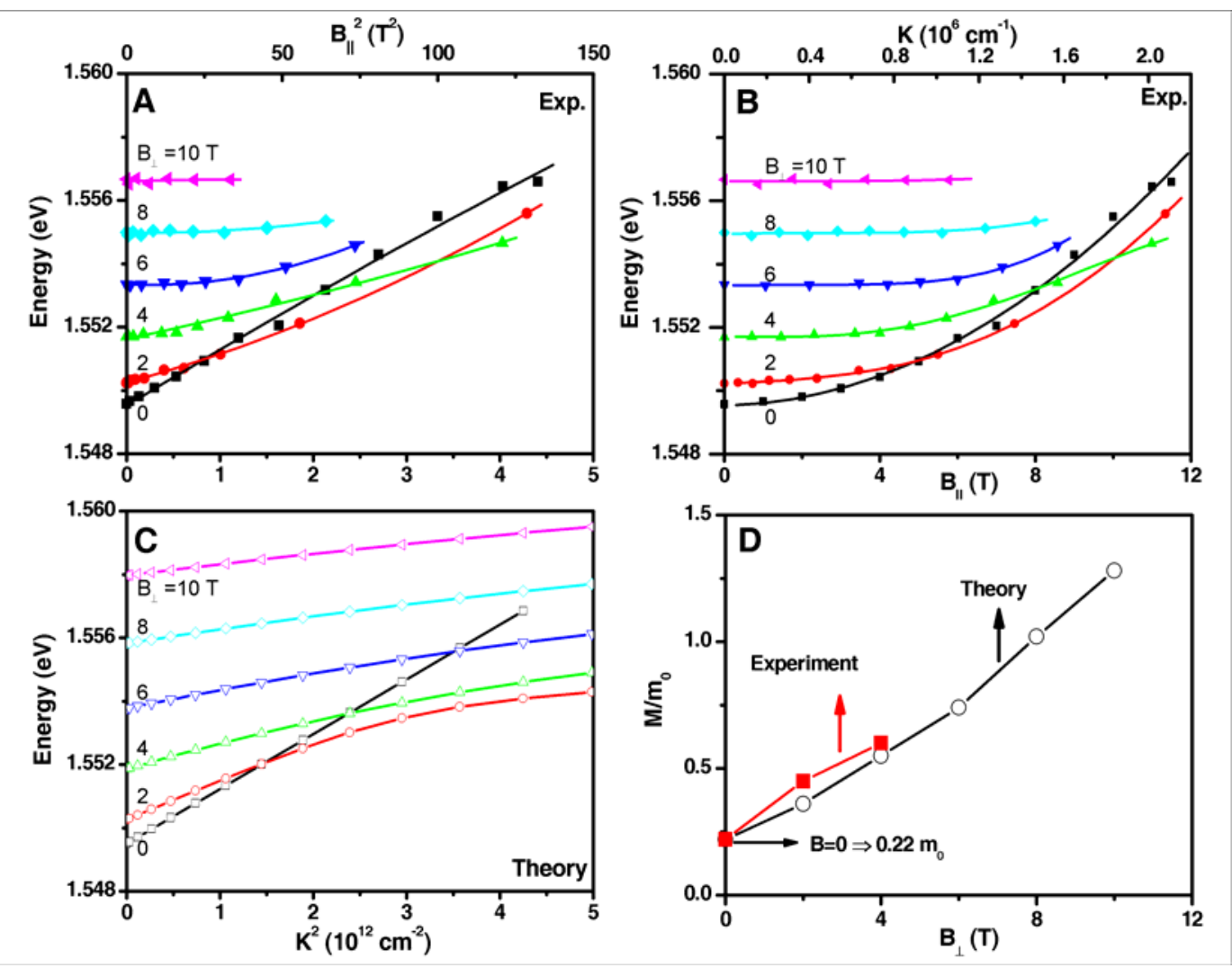

Figure 4.18 (A) Measured and (B) Calculated magnetoexciton dispersions at $\mathrm{B} \perp=0,2,4,6,8$ and $10 \mathrm{~T}$ versus $\mathrm{K}^{2}\left(\mathrm{~B}_{\|}{ }^{2}\right)$. (C) Measured dispersions 
versus $\mathrm{K}\left(\mathrm{B}_{\|}\right)$. (D) Measured (solid squares) and calculated (open circles) magnetoexciton effective mass at the band bottom versus $B_{\perp}$.

As the in-plane magnetic field component $\mathrm{B}_{\|}$increases, the energy of indirect magnetoexcitons increase due to the displacement of the dispersion surface in momentum space, while the energies of the direct transitions are practically unchanged. The magnitude of the PL energy shift is smaller for the higher perpendicular field component $\left(\mathrm{B}_{\perp}\right)$. The $\mathrm{PL}$ line shift versus $\mathrm{B}_{\|}$determines the magnetoexciton dispersion. This is shown in Figure 4.18A and $\mathrm{C}$ for various $\mathrm{B}_{\perp}$. The magnetoexciton effective mass at the band bottom is determined by quadratic fits to the dispersion curves at small $\mathrm{K}$. At $\mathrm{B}_{\|}=0$ the measured dispersion corresponds to an effective mass, $\mathrm{M}=0.22 \mathrm{~m}_{0}$, where $\mathrm{m}_{0}$ is the electron rest mass. This value is in good agreement with the calculated mass of heavyhole exciton in GaAs QWs $\sim 0.25 \mathrm{~m}_{0}$ (electron effective mass $\mathrm{m}_{\mathrm{e}}=0.067 \mathrm{~m}_{0}$ and the heavy-hole mass for in-plane motion, $\left.\mathrm{m}_{\mathrm{hh}_{\|}} \sim 0.18 \mathrm{~m}_{0}[173,174]\right)$.

Drastic changes of the magnetoexciton dispersion are observed at finite $\mathrm{B}_{\perp}$. The dispersion curves flatten at small momenta as seen in Figure 4.18. This corresponds to a strong enhancement of the magnetoexciton mass: At $\mathrm{B}_{\perp}=4 \mathrm{~T}$, the magnetoexciton mass has increased by about a factor of three. At higher $\mathrm{B}_{\perp}$, the dispersion becomes so flat that the scattering of the experimental points does not allow precise determination of the mass due to limit of the available magnetic field and experimental accuracy. The experimental results are now compared with $a b$ initio calculations $[164,172]$. The results are shown in Figure 4.18B. We find that in a finite magnetic field the effective mass which characterizes the dispersion at small $\mathrm{P}$ increase monotonically with $\mathrm{B}_{\perp}$. The calculated 
effective mass enhancement is in good agreement with the experimental data. ${ }^{18}$ The agreement remains qualitatively good at high momenta; however, the theory gives a rate of energy increase with momentum at high P slower that the experiment. This difference could be accounted for by including details such as the effects of the finite QW thickness and finite radiative zone width (i.e. finite $\mathrm{X}$ line width). Note also that due to the line broadening and intensity reduction, at high $\mathrm{B}_{\|} \geq 10 \mathrm{~T}$, the experimental accuracy for the

peak X energy decreases. We note that the observed large enhancement of the effective mass of the indirect exciton in magnetic fields is a single-exciton effect contrary to the well known renormalization effects in neutral and charged e-h plasmas [76].

In conclusion, we find that the exciton effective mass is strongly enhanced as $\mathrm{B}_{\perp}$ increases and, becomes overwhelmingly larger than the sum of electron and hole masses at high fields. This experimental finding proves that the magnetoexciton is a quasiparticle whose effective mass is tunable by magnetic fields and determined by the coupling between the CM motion and internal structure rather than by the masses of its constituents.

\subsection{Summary}

In this chapter, we described fundamental properties of spatially indirect-Xs. We reported the time-integrated and time-resolved photoluminescence spectroscopy as a function of temperature (T), bias voltage (electric field, $V_{b}$ ), and laser excitation power $\left(P_{\text {laser }}\right)$. Methods used to estimate $\mathrm{X}$ density were also discussed. Finally, we studied the

\footnotetext{
18 The only fitting parameter in the calculation is effective mass at $\mathrm{B}_{\perp}$ which was obtained from the measured indirect- $X$ dispersion: $\mathrm{M}=0.22 \mathrm{~m}_{0}$.
} 
dispersion and effective mass of indirect-Xs measured by applying magnetic fields parallel to the quantum well plane. 


\section{Chapter 5 Confined Exciton Systems}

\subsection{Introduction}

Degenerate exciton systems have been produced in quasi-two-dimensional confined areas in semiconductor coupled quantum well structures. We observed contractions of clouds containing tens of thousands of excitons (Xs) within areas as small as $(10 \mu \mathrm{m})^{2}$ near 10 Kelvin. Collection of these confined cold dense indirect-X systems was first reported in ref. [44]. We present here a phase diagram of X systems confined in these small areas, which for brevity we refer to as 'traps'. The spatial and energy distributions of optically active Xs in these traps were determined by measuring photoluminescence (PL) as a function of temperature $(T)$ and laser excitation $\left(P_{\text {laser }}\right)$ and were used as thermodynamic quantities to construct the phase diagram of the $\mathrm{X}$ system, which demonstrates the existence of distinct phases. We will compare the spatiallyresolved PL ( $\mu$-PL) imaging and spectroscopy under a uniform or remote local laser excitation. Then we will present the time-resolved photoluminescence (TRPL) measurements, which reveal partially the formation mechanisms of these degenerate confined X systems. To clarify the formation mechanisms, more extensive experiments will be detailed later in Chapter 7 .

\subsection{Effects of confinement}

The quasi-two-dimensional (quasi-2D) nature of coupled quantum wells (CQW) raises important theoretical issues related to phase transitions in reduced dimensionality. 
BEC only occurs at $\mathrm{T}=0$ in a homogeneous $2 \mathrm{D}$ system of ideal bosons $[40,175,41]$, but can occur at finite $\mathrm{T}$ when they are confined in $2 \mathrm{D}$ traps $[42,43]$. However, for non-ideal interacting bosons, such as Xs, no general result is known. Theoretically, it has been advanced [176-179] that in the case of contact interaction and harmonic-2D traps no BEC occurs at finite temperature although there is a strong particle concentration at the trap center below the $T_{c}$ calculated for ideal bosons. Experimentally, the MIT atomic BEC group has demonstrated realization of BEC of sodium atoms in lower dimensions [180]. Producing local confined cold X systems may also be a key step to realizing excitonic BEC in quasi-2D structures. In that respect, applying the $T_{c}$ formulae for ideal bosons in a square 2D-box [43] to the confined Xs observed in GaAs/AlGaAs CQW gives $T_{c} \approx 1 \mathrm{~K}$ for reachable experimental conditions.

\subsection{Uniform excitation}

Samples containing multiple mesas of various sizes are processed from the same wafer. A low density, approximately one per $(500 \mu \mathrm{m})^{2}$ area, of the 'traps' were found throughout the samples examined. Although we elaborate here the experiments performed on a $(780 \mu \mathrm{m})^{2}$ mesa, similar observations are made on different samples processed from the same wafer grown by a MBE machine. For the experiments reported in this chapter, a static bias voltage $V_{b}=-0.95 \mathrm{~V}(\approx 23 \mathrm{kV} / \mathrm{cm})$ was applied to the CQW sample unless specified otherwise. The mesa was excited uniformly by a defocused $\mathrm{HeNe}$ laser with a spot size $\sim 600-700 \mu \mathrm{m}$ (Full Width at Half Maximum) centered on one of the two traps present in the mesa. Two series of experiments were performed: (i) PL imaging by a CCD camera with a $10 \mathrm{~nm}(\sim 21 \mathrm{meV})$ band-pass interference filter centered at the indirect-X emission line $-\mathrm{a} \sim 2 \mu \mathrm{m}$ spatial resolution is achieved by the combination of a 
microscope objective and camera lens, and (ii) diffraction limit $(\sim 1.7 \mu \mathrm{m})$ spatiallyresolved PL spectra obtained by scanning a pinhole in the confocal image plane. The PL imaging provides direct information on the spatial distribution of the Xs within the filter bandwidth; whereas the PL scans determine the local $(\sim 1.7 \mu \mathrm{m})$ energy distribution of the indirect-Xs with a $\sim 0.1 \mathrm{meV}$ resolution.

A CCD PL image of a $(780 \mu \mathrm{m})^{2}$ mesa under a uniform HeNe laser excitation is shown in Figure 5.1. This mesa contains two natural 'traps' separated by approximately $280 \mu \mathrm{m}$, which are labeled as Trap-A and Trap-B. In the following sections, we detail a phase diagram obtained for Trap-A.

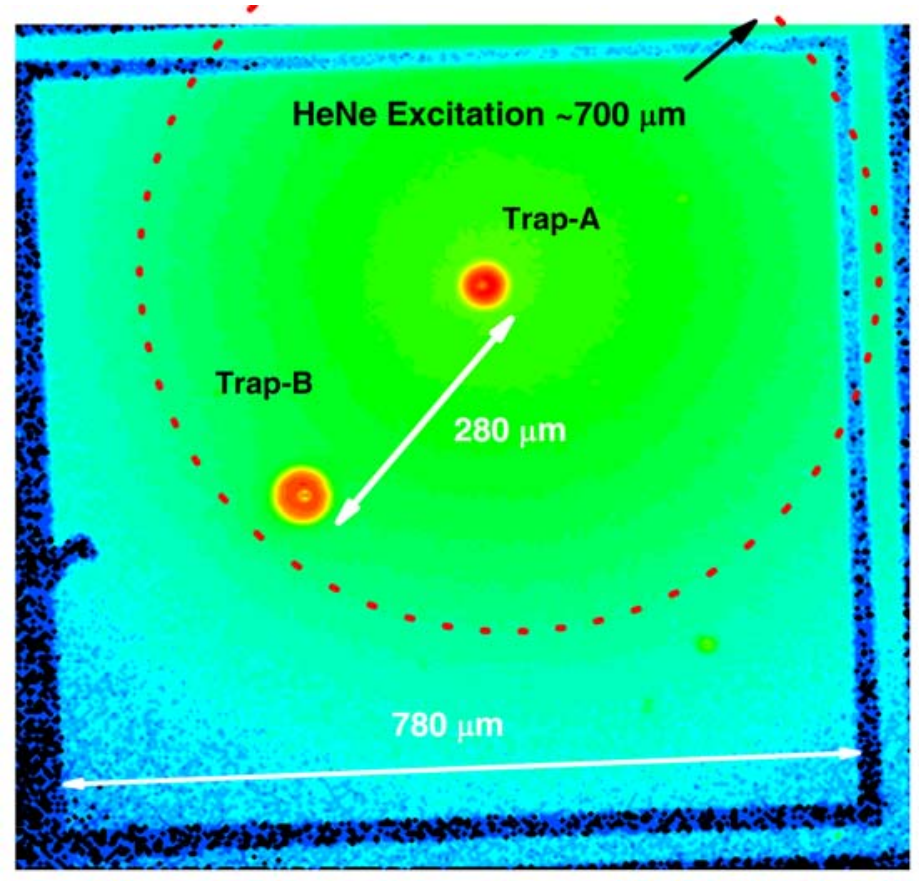

Figure 5.1 A false color CCD PL image of a $780 \mu \mathrm{m}$ mesa with two natural 'traps'. This example image is taken at $T=1.7 \mathrm{~K}$ with a defocused uniform HeNe laser of spot diameter $\sim 600-700 \mu \mathrm{m}$ and $P_{\text {laser }}=1.25 \mathrm{~mW}$ centered at Trap-A. The dark square edges are the $\mathrm{Au} / \mathrm{Pd} / \mathrm{Ge}$ alloy contacts on the top of the mesa. 


\subsubsection{Qualitative descriptions}

The PL images of the indirect-X cloud for three temperatures $T=1.7 \mathrm{~K}, 8 \mathrm{~K}$ and $20 \mathrm{~K}$ (Figure 5.2A) at a low and uniform laser excitation power, $P_{\text {laser }}=50 \mu \mathrm{W}$ show that contraction of the $\mathrm{X}$ cloud is evident with decreasing bath temperature: The cloud radius decreases more than an order of magnitude to $\sim 10 \mu \mathrm{m}$, whereas the peak intensity increases by a factor of 50. The 2D PL images at $T=1.7 \mathrm{~K}$ (Figure 5.2B) for three $P_{\text {laser }}$ values show that with increasing $P_{\text {laser, }}$ the $X$ cloud first contracts and then expands, exhibiting an annular shape with a darker central region that also shrinks and then expands with $P_{\text {laser. }}$ The color bar scale on the figure shows the local density of Xs estimated from the PL intensity by the methods described below. From these images we deduce the spatially integrated PL intensity $\left(\mathrm{I}_{\mathrm{PL}}\right)$, the root mean square radius $\left(\sigma_{\mathrm{PL}}\right)$, the skewness (asymmetricity), and kurtosis ( $\mathrm{K}_{\mathrm{PL}}$ - concentration or peakedness as compared with a Gaussian distribution) ${ }^{19}$. These parameters are used as thermodynamic quantities to characterize the $\mathrm{X}$ fluid.

Spatially resolved PL spectra were also measured as a function of $T$ and $P_{\text {laser }}$ by scanning a pinhole in the confocal image plane. The energy-space hybrid 3D plots shown in Figure 5.3 are two example results at $T=1.7 \mathrm{~K}$ and $20.6 \mathrm{~K}$, respectively.

\footnotetext{
19 The spatial moments of the PL images were calculated with a modified java plug-in module of the ImageJ, a free image processing program from NIH. Definitions of these quantities can be easily obtained by extending the moments defined in Sec. 4.3 to two dimensions.
} 


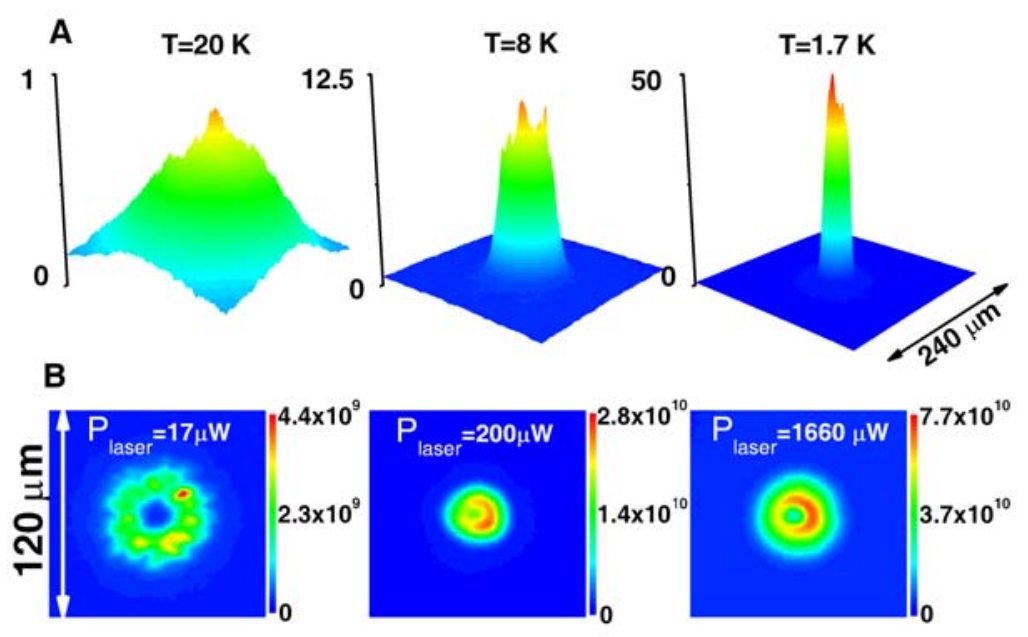

Figure 5.2 Photoluminescence as a function of temperature $(T)$ and laser excitation $\left(P_{\text {laser }}\right)$ in selected images. (A) T-dependent PL 3D false-color images of the confined excitons (Xs) at $T=1.7 \mathrm{~K}, 8 \mathrm{~K}, 20 \mathrm{~K}$ with $P_{\text {laser }}=$ $50 \mu \mathrm{W}$ (laser excitation spot size $\sim 700 \mu \mathrm{m}$ ). PL intensities are rescaled as indicated on the vertical axis. The $\sigma_{P L}$ deduced from the spatial distributions are $\sigma_{\mathrm{PL}}=47,21$, and $10 \mu \mathrm{m}$, respectively, and the $\mathrm{K}_{\mathrm{PL}}=$ $0.45,0.03$, and +1.19 showing that the $\mathrm{X}$ cloud is spatially more peaked than a Gaussian. (B) $P_{\text {laser-dependent }}$ PL 2D false-color images of confined Xs at $T=1.7 \mathrm{~K}$ for $P_{\text {laser }}$ as indicated. The estimated optically active $\mathrm{X}$ density is represented as colorbar scales. From left to right, $\sigma_{\mathrm{PL}}$ are 19,9 , and $13 \mu \mathrm{m}$ respectively, and $\mathrm{K}_{\mathrm{PL}}$ are $+0.33,+1.47$, and +1.20 .
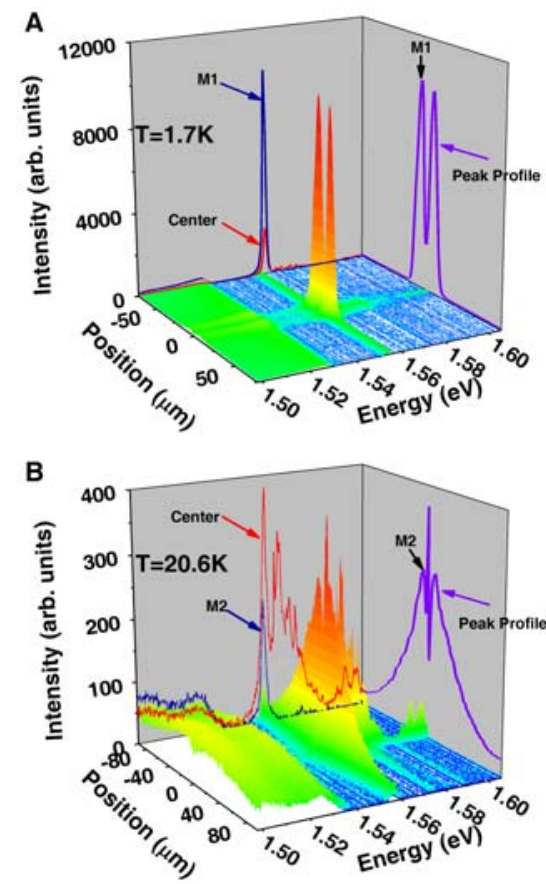

Figure 5.3 Spatially-resolved PL spectra across Trap-A under a uniform HeNe excitation $\left(P_{\text {laser }}=198 \mu \mathrm{W}\right)$ at $\mathrm{T}=1.7 \mathrm{~K}$ and $20.6 \mathrm{~K}$. 


\subsubsection{Phase diagram: $\mu$-PL imaging}

The phase diagram of $\sigma_{\mathrm{PL}}\left(T, P_{\text {laser }}\right)$ is shown in Figure 5.4. At either high temperature or low excitation intensity the $\mathrm{X}$ cloud is extended. Both low temperature and a significant excitation are required for Xs to be concentrated in a small area. Indeed, $\sigma_{\mathrm{PL}}$ is at its minimum $(\sim 10 \mu \mathrm{m})$ for $T<4 \mathrm{~K}$ and moderate excitation $\left(P_{\text {laser }} \approx 35 \mu \mathrm{W}\right.$ to $500 \mu \mathrm{W})$. In that range, the kurtosis phase diagram $\mathrm{K}_{\mathrm{PL}}(T$, Plaser) (Figure 5.5) demonstrates that the $\mathrm{X}$ distribution is more concentrated than a Gaussian, despite the central intensity dip. It is noteworthy that $\sigma_{\mathrm{PL}}$ exhibits steepest variations in a narrow domain - nearly vertical for $P_{\text {laser }} \leq 20 \mu \mathrm{W}$ and diagonal for $P_{\text {laser }} \geq 20 \mu \mathrm{W}$ around $\sigma_{\mathrm{PL}}=$ $30 \mu \mathrm{m}$, which reveals the existence of distinct phases. At fixed $T \leq 10 \mathrm{~K}$, as $P_{\text {laser }}$ is increased, the X cloud first contracts when $P_{\text {laser }}$ crosses $\sim 20 \mu \mathrm{W}$, plateaus over a small range of moderate intensities, then expands. At fixed $P_{\text {laser }}$, as $T$ decreases, the $\mathrm{X}$ cloud contracts and experiences a sharp reduction in size near $T=10 \mathrm{~K}$. This behavior is seen more clearly in $T$-dependent cuts of $\sigma_{\mathrm{PL}}$ for five $P_{\text {laser }}$ between $25 \mu \mathrm{W}$ and $6 \mathrm{~mW}$ (Figure 5.4B). For $P_{\text {laser }}=25 \mu \mathrm{W}, \sigma_{\mathrm{PL}}(T)$ exhibits a clear kink near $T=10 \mathrm{~K}$, which confirms the sudden cloud contraction. However, that kink becomes less pronounced at higher $P_{\text {laser }}$ as $\sigma_{\mathrm{PL}}$ becomes progressively $T$ independent. This transition is further supported by the corresponding normalized $\mathrm{I}_{\mathrm{PL}}(T)$ curves (Figure 5.4C), which, at low excitation, have a strong $T$ dependence with an almost steplike increase between $T=15 \mathrm{~K}$ and $10 \mathrm{~K}$. As the excitation density increases, the magnitude of $\mathrm{IPL}_{\mathrm{PL}}(T)$ increases by a factor of 100 but its $T$ dependence becomes progressively flatter. 

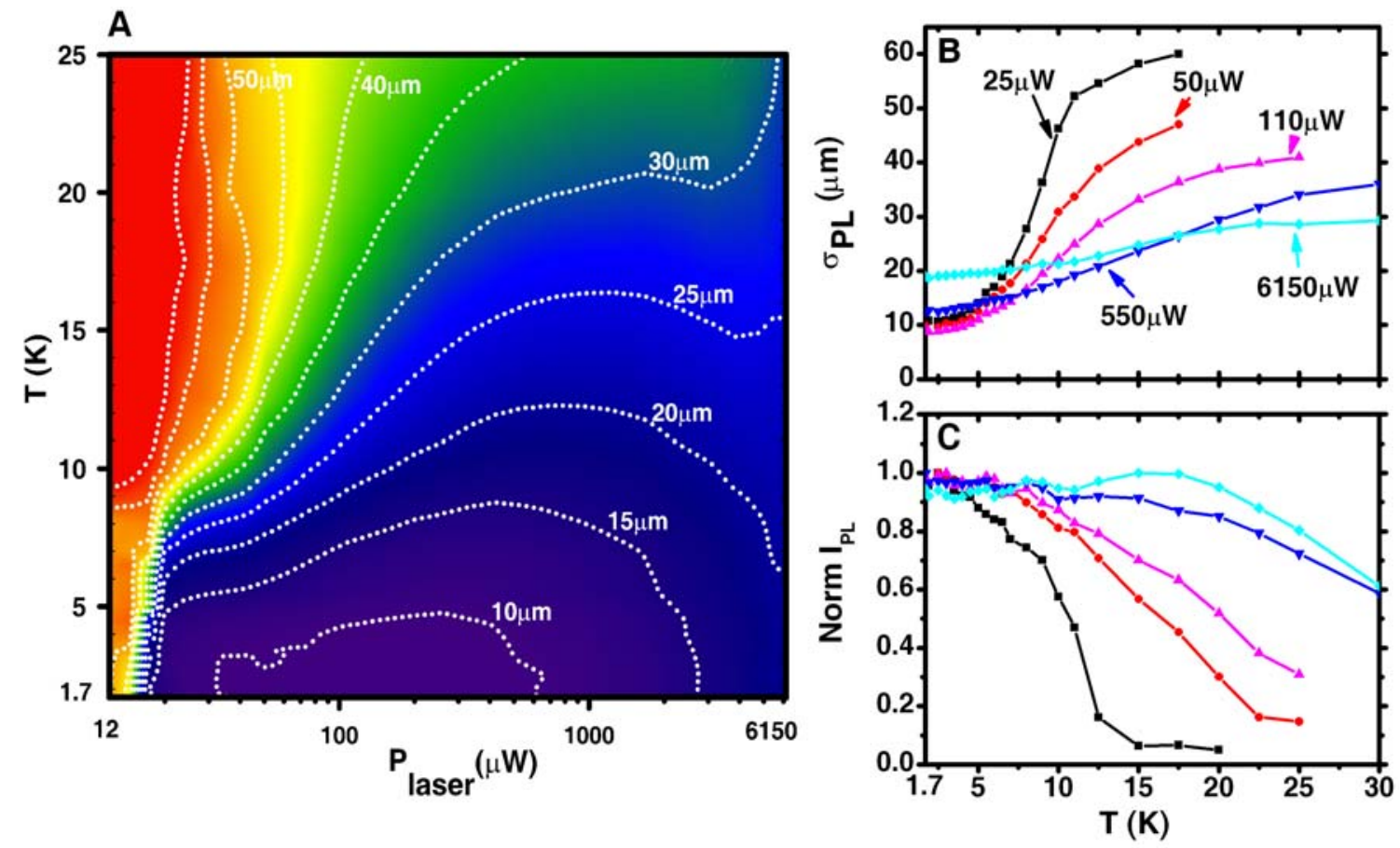

Figure 5.4 (A) Full phase diagram of the root-mean-square radius of $\mathrm{X}$ cloud, $\sigma_{P L}\left(T, P_{\text {laser }}\right)$. The white contour lines highlight the cloud extension from $10 \mu \mathrm{m}$ to $60 \mu \mathrm{m}$ in 5- $\mu \mathrm{m}$ steps. (B) Cross sections, $\sigma_{\mathrm{PL}}(T)$, of the Fig. 2A phase diagram for fixed laser excitation intensities for $P_{\text {laser }}$ as indicated (C) $T$-dependent normalized spatially integrated PL $\left[\operatorname{I}_{\mathrm{PL}}(T)\right]$. $\mathrm{I}_{\mathrm{PL}}(T)$ rises sharply below $T \approx 10 \mathrm{~K}$ for low $P_{\text {laser }}$.

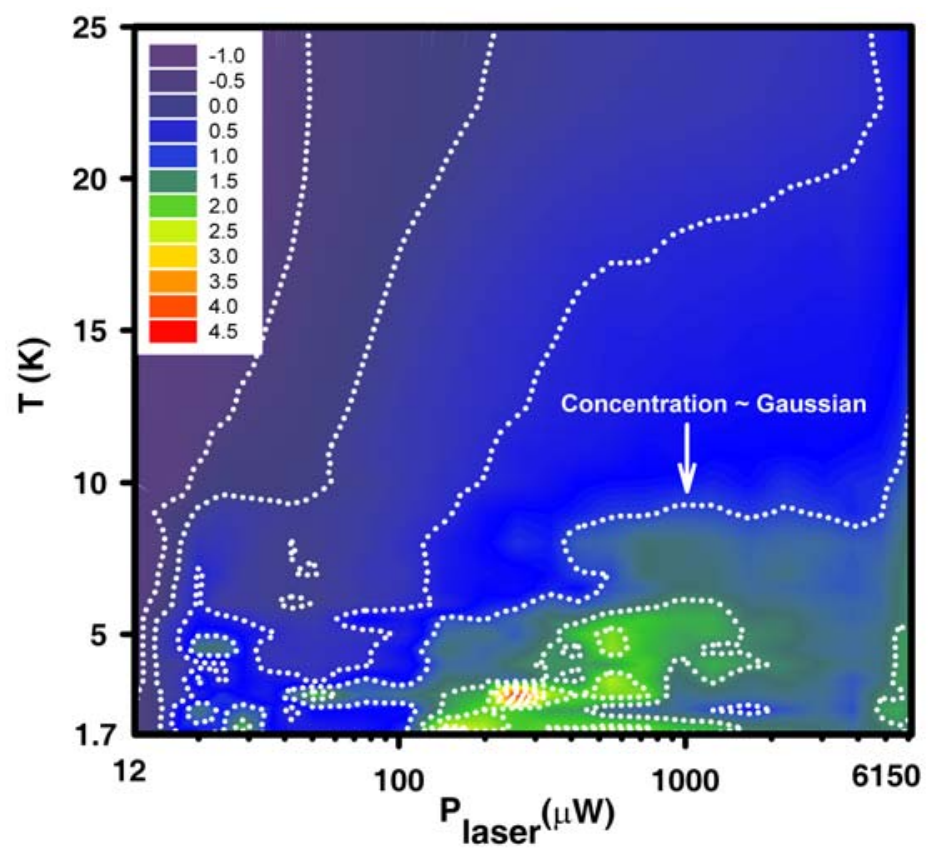

Figure 5.5 Kurtosis $\left(\mathrm{K}_{\mathrm{PL}}\right)$ phase diagram of the $X$ cloud. $\mathrm{K}_{\mathrm{PL}}=1$ for a Gaussain distribution and $\mathrm{K}_{\mathrm{PL}}>1$ for a more concentrated distribution. For 
low $T$ and moderate $P_{\text {laser }}$ where the $\mathrm{X}$ clouds are most localized $\left(\sigma_{\mathrm{PL}} \leq 10\right.$ $\mu \mathrm{m}$ ), the $\mathrm{X}$ clouds are also most concentrated as $\mathrm{K}_{\mathrm{PL}}>1$.

\subsubsection{Phase diagram: $\mu$-PL spectroscopy}

The PL intensity is only sensitive to the radiative recombination of optically active, bound or unbound electron-hole pairs with center of mass momentum $\mathrm{K} \approx 0$ $[100,99]$. It may not reflect the X density which, for CQW Xs, is more directly related to the PL peak energy $\left(\mathrm{E}_{0}\right)$ blue shift due to the indirect-X interaction (see Sec. 4.5.1). Therefore, to better characterize the energy distribution of the indirect-X systems, we used the PL scans that provide local spectroscopic information with a $\Delta y \approx 1.7 \mu \mathrm{m}$ spatial resolution (Figure 5.6A).

\subsubsection{Excitation intensity dependence}

In Figure 5.6B, the black curve gives the indirect-X PL intensity profile, $\mathrm{I}_{\mathrm{X}}(\mathrm{y})$ (spectrally integrated over the indirect-X peak, see Sec. 4.3), for $P_{\text {laser }}=200 \mu \mathrm{W}$ and $T=$ 1.7 K. The PL peak intensity profiles, $\mathrm{I}_{0}(\mathrm{y})$, for four $P_{\text {laser }}$ values are also shown in Figure 5.7A. Consistent with the annular structure of the Figure 5.2B images, $\mathrm{I}_{\mathrm{X}}(\mathrm{y})$ exhibits a central dip surrounded by two intense maxima. The $\mathrm{E}_{0}$ profile (magenta curve) shows that the blue shift increases monotonically from the cloud edges to a maximum at the center. Thus, the indirect-Xs at the cloud center are dense but hot $(K>0)$, not as optically active as those cold $(\mathrm{K} \approx 0)$ ones in the bright annulus. This is consistent with the variations of the PL linewidth $\Gamma(\mathrm{y})$ (red curve), which narrows sharply where $\mathrm{I}_{\mathrm{X}}(\mathrm{y})$ is maximal and broadens a little at the center. This behavior is observed in the region where the indirect$\mathrm{X}$ cloud is concentrated. But for lower $P_{\text {laser }}$ (less than about $100 \mu \mathrm{W}$ ), the $\mathrm{E}_{0}$ profile with discontinuities first appears [44], and then near the center $\mathrm{E}_{0}$ gradually flattens (Figure 5.7B). 

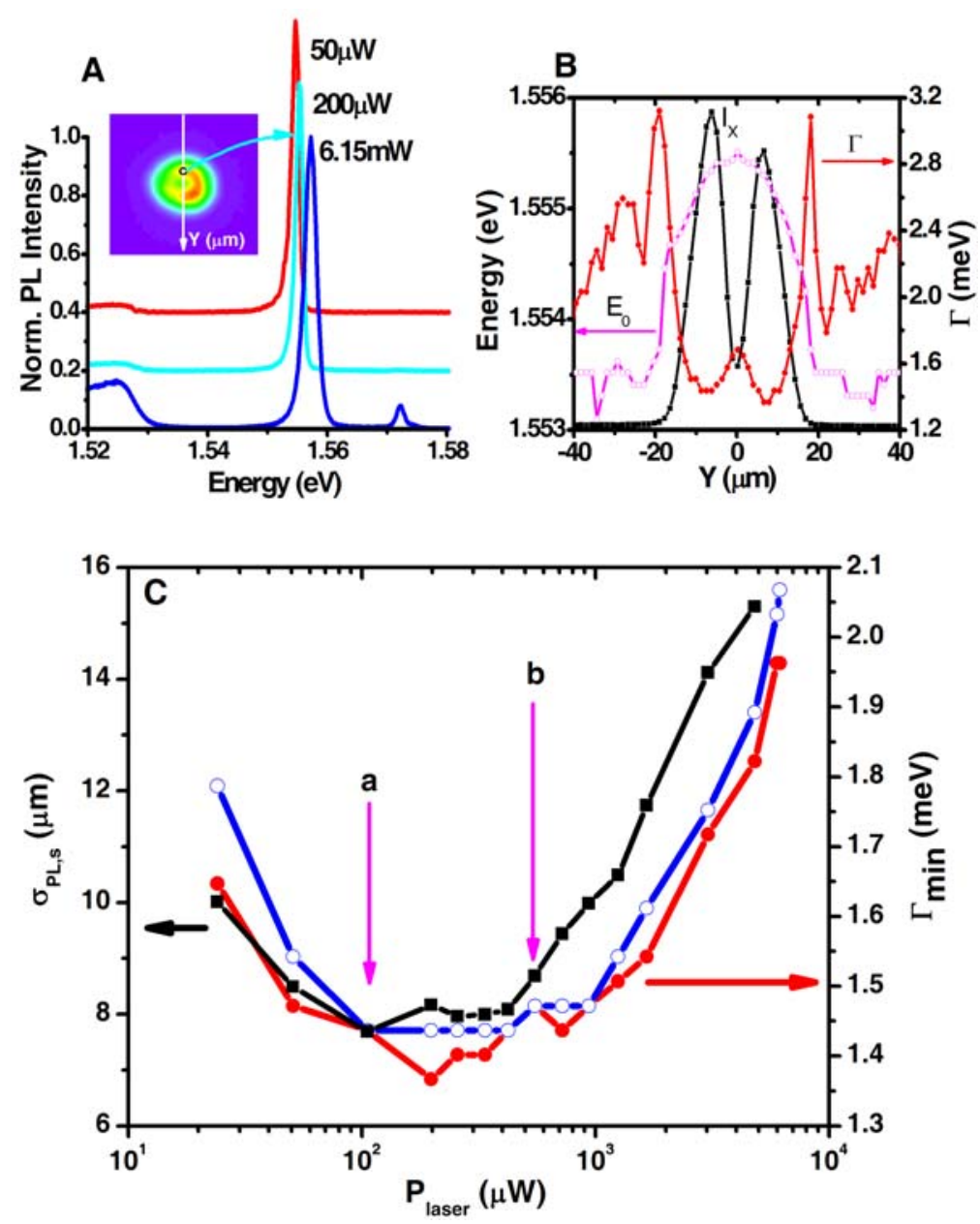

Figure 5.6 Analysis of spatially resolved spectra. (A) Selected PL spectra for $P_{\text {laser }}$ as indicated. The inset 2D PL image shows the path (white arrow) of a pinhole scanning across the trap to measure these spectra. The PL spectra shown are measured at the position (black circle) where the PL intensity is at a maximum. The spectra are normalized and vertically offset for clarity. The low-energy PL emission $(\mathrm{E}<1.53 \mathrm{eV})$ is from $n^{+}$-GaAs layer, and the extra PL peak at $\mathrm{E}=1.572 \mathrm{eV}$ is from direct excitons. (B) Spatial profiles of spectrally integrated X PL $\left(\mathrm{I}_{\mathrm{X}}\right)$ [black curve], $\Gamma$ [red curve], and peak energy $\left(E_{0}\right)$ obtained by analyzing scanned spectra at all positions for $T=1.7 \mathrm{~K}$ and $P_{\text {laser }}=200 \mu \mathrm{W}$. The locations of minimum linewidth $\left(\Gamma_{\min }\right)$ and maximum PL intensity coincide. (C) Two sets of $\Gamma_{\min }$ values (blue and red curves) and cloud radii, $\sigma_{\mathrm{PL}, \mathrm{S}}$ (black curve), versus $P_{\text {laser }}$ at $T=1.7 \mathrm{~K}$. The $\mathrm{X}$-cloud radii are deduced from profiles of $\mathrm{I}_{\mathrm{X}}(\mathrm{y})$. The $\Gamma_{\min }\left(P_{\text {laser }}\right)$ and $\sigma_{\mathrm{PL}, \mathrm{S}}\left(P_{\text {laser }}\right)$ curves have parallel variations, revealing three regimes: (I) a dilute X-system, (II) a degenerate condensed X fluid with narrowest $\Gamma_{\min }$ and most concentrated cloud, and (III) a hybrid X/e-h phase. Arrows (a) and (b) indicate the approximate boundaries of regime (II), where the estimated maximum $X$ density are (a) $1.7 \times 10^{10}$ and (b) $4.6 \times 10^{10} \mathrm{~cm}^{-2}$, respectively. 


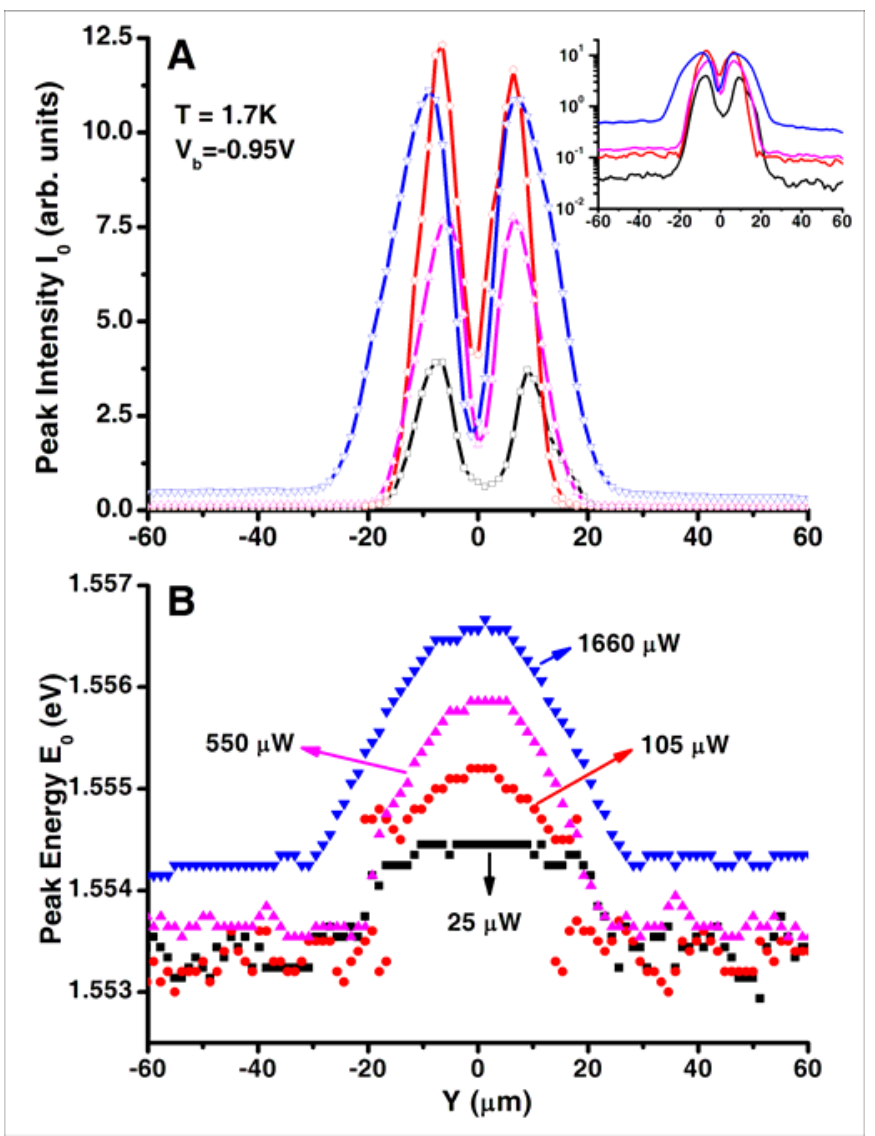

Figure 5.7 Spatially-resolved indirect-X PL profiles for $P_{\text {laser }}$ as indicated. (A) Inidrect-X PL peak intensities $\left(\mathrm{I}_{0}\right)$ in linear and log scales (inset). (B) Inidrect-X PL peak energies $\left(\mathrm{E}_{0}\right)$.

The $\mathrm{I}_{\mathrm{X}}(\mathrm{y})$ mean square radius, $\sigma_{\mathrm{PL}, \mathrm{S}}$, and the PL linewidths at the two minima, $\Gamma_{\min }$, are plotted versus $P_{\text {laser }}\left(\right.$ Figure $5.6 \mathrm{C}$ ) for $T=1.7 \mathrm{~K}$. The $\sigma_{\mathrm{PL}, \mathrm{S}}\left(P_{\text {laser }}\right)$ curve agrees with the behavior seen in Figure 5.4A, i.e., the $X$ cloud contracts to a small area, $\sigma_{\mathrm{PL}, \mathrm{S}} \approx 7$ $\mu \mathrm{m}$, at low $T$ for moderate $P_{\text {lase }}$. Interestingly the linewidth $\Gamma_{\min }\left(P_{\text {laser }}\right)$ curves show a parallel variation versus $P_{\text {laser }}$. At low $T$ where phonons are scarce, the low-density PL linewidth in a QW is dominated by disorder-related inhomogeneous broadening and is roughly constant, then it usually increases with the density due to the $\mathrm{X}-\mathrm{X}$ interaction, $\mathrm{X}$ carrier scattering and screening. In the case of indirect-Xs, however, the repulsive dipoledipole interaction screens the in-plane potential fluctuations, an effect particularly pronounced in the statistically degenerate regime [39]. Figure 5.6C reveals three regimes: 
(I) $20 \mu \mathrm{W} \leq P_{\text {laser }} \leq 100 \mu \mathrm{W}$, (II) $100 \mu \mathrm{W} \leq P_{\text {laser }} \leq 500 \mu \mathrm{W}$, and (III) $500 \mu \mathrm{W} \leq P_{\text {laser. }}$ In regime I and II, the indirect-X PL peak is narrow $(\Gamma<2 \mathrm{meV})$, although it broadens a little near the center (see Figure 5.6B). This narrow linewidth confirms the well-defined X distribution. In regime III, the PL spectra near the center have significantly broad indirect-X peaks and stronger high-energy emissions, indicative of the presence of e-h plasmas. To estimate the density of Xs we combine two methods: (i) measurements of the PL peak blue shift and (ii) careful measurements and calibration of the photon collection efficiency of the PL imaging system (see Sec. 4.5). The densities corresponding to the crossover between these regimes indicated by the arrows in Figure 5.6C are, respectively, $n_{X} \approx 1.7 \times 10^{10} \mathrm{~cm}^{-2}$ and $n_{X} \approx 4.6 \times 10^{10} \mathrm{~cm}^{-2}$, still below the Mott density $[31,129], n_{\text {Mott }} \approx$ $10^{11} \mathrm{~cm}^{-2}$ estimated from $n_{\text {Mott }} \times \mathrm{a}_{\mathrm{X}} \approx 1$ [115]. Regime I corresponds to a dilute $\mathrm{X}$ system, whereas regime III is a hybrid X/e-h phase, where Xs may not be well defined and effects of Fermi statistics cannot be neglected. In regime II, however, simultaneously the linewidth is the narrowest and the $\mathrm{X}$ cloud is the most concentrated with size depending moderately on $P_{\text {laser }}$ There the number of Xs in the X cloud varies between about $10^{4}$ and $10^{5}$, which indicates a highly statistically degenerate phase [44]. Thus, a dense and cold X fluid forms in regime II where the environmental fluctuations are smoothed out and the effects of scattering reduced or even suppressed. It is noteworthy that the linewidth dependence $\Gamma_{\min }\left(P_{\text {laser }}\right)$ across the trap and the $\Gamma\left(P_{\text {laser }}\right)$ measured at the excitation spot (Sec. 4.3.2) exhibit a similar trend.

\subsubsection{Temperature dependence}

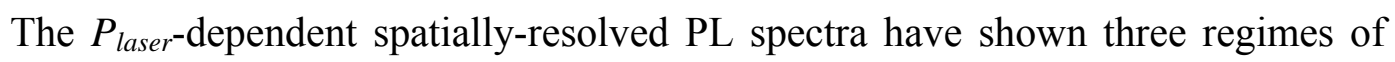
interest. Therefore, we further measured T-dependent spatially-resolved PL for four 
values of $P_{\text {laser }}-50,200,550$, and $1660 \mu \mathrm{W}$ - corresponding to regime I, II, and III described previously.

The T-dependent spatial profiles of X PL integrated intensities $\left(\mathrm{I}_{0}\right)$, linewidths $(\Gamma)$, peak energies $\left(E_{0}\right)$, and average energies $\left(E_{X}\right)$ are shown for $P_{\text {laser }}=200 \mu \mathrm{W}$ in Figure 5.8A to D, respectively. The $\mathrm{X}$ cloud expands with increasing $T$ while maintaining an overall annular shape. Near the center of the trap $(|y|<3 \mu \mathrm{m})$, we observed broad PL emission lines with a lower peak energy but a high energy component. These broad PL emissions cause the 'spikes' to appear in the figures. They are more pronounced compared with the narrow X lines at a high $T$ (see Figure 5.3 for example PL spectra) and a high $P_{\text {laser }}$. These broad PL emissions at the center could be due to the presence of e-h plasma or joule heating caused by a current tunneling through the trap center.

The $\mathrm{E}_{0}(\mathrm{y})$ profile at $T=1.7 \mathrm{~K}$ displays discontinuities at $|\mathrm{y}| \approx 20 \mu \mathrm{m}$, where the PL emissions exhibit two peaks - one is the continuation of the inner high-energy PL lines, and the other is the continuation of the outer low-energy PL lines. In determining the linewidth, these two overlapping lines were not separated, and thus an increase of $\Gamma$ is seen in Figure 5.8B. These discontinuities were washed out (i.e. PL line splitting disappeared) at $T \approx 5-10 \mathrm{~K}$. Note that the above mentioned phenomena were only observed for a small range of low $P_{\text {laser }}(\sim$ regime I) as discussed in the previous section.

The overall $\mathrm{X}$ energy profiles, including $\mathrm{E}_{0}(\mathrm{y})$ and $\mathrm{E}_{\mathrm{X}}(\mathrm{y})$, experience unusual blue shifts with increasing $\mathrm{T}$. We will discuss this in the following section by comparing the PL spectra near the trap center and remote spots.

The PL linewidth $\Gamma(\mathrm{y})$ narrows near where $\mathrm{I}_{\mathrm{X}}(\mathrm{y})$ is maximal and broadens at the center. The $T$-dependent minimumal linewidth $\Gamma_{\min }(\mathrm{T})$ curves are shown in Figure 5.9B 
for $P_{\text {laser }}$ values as indicated. For moderate and high $P_{\text {laser }}$ values, $\Gamma_{\min }$ increases linearly with $T$ at a similar rate; whereas for low $P_{\text {laser, }}$, it increases more rapidly with $T$ and experiences a reduction near $\sim 7-10 \mathrm{~K}$. To understand if the broadening is mainly due to the scattering with longitudinal acoustical (LA) phonons [145], we measured the $T$ dependent direct-X PL at a remote location away from any trap at $V_{b}=0 \mathrm{~V}$ under a uniform excitation. The direct-X linewidth increases at a much slower rate with $T$. This and the unusual $\Gamma_{\min }(T)$ curves for low $P_{\text {laser values suggest that, in additon to scattering }}$ with LA phonons, other broadening mechanisms are involved. The $\mathrm{X}$ cloud radii, $\sigma_{\mathrm{PL}, \mathrm{S}}$, are also calculated from the $\mathrm{I}_{\mathrm{X}}(\mathrm{y})$ profiles (Figure 5.8A). The $T$-dependent $\sigma_{\mathrm{PL}, \mathrm{S}}$ curves shown in Figure 5.9 are consistent with those measured by PL images (Figure 5.4B).
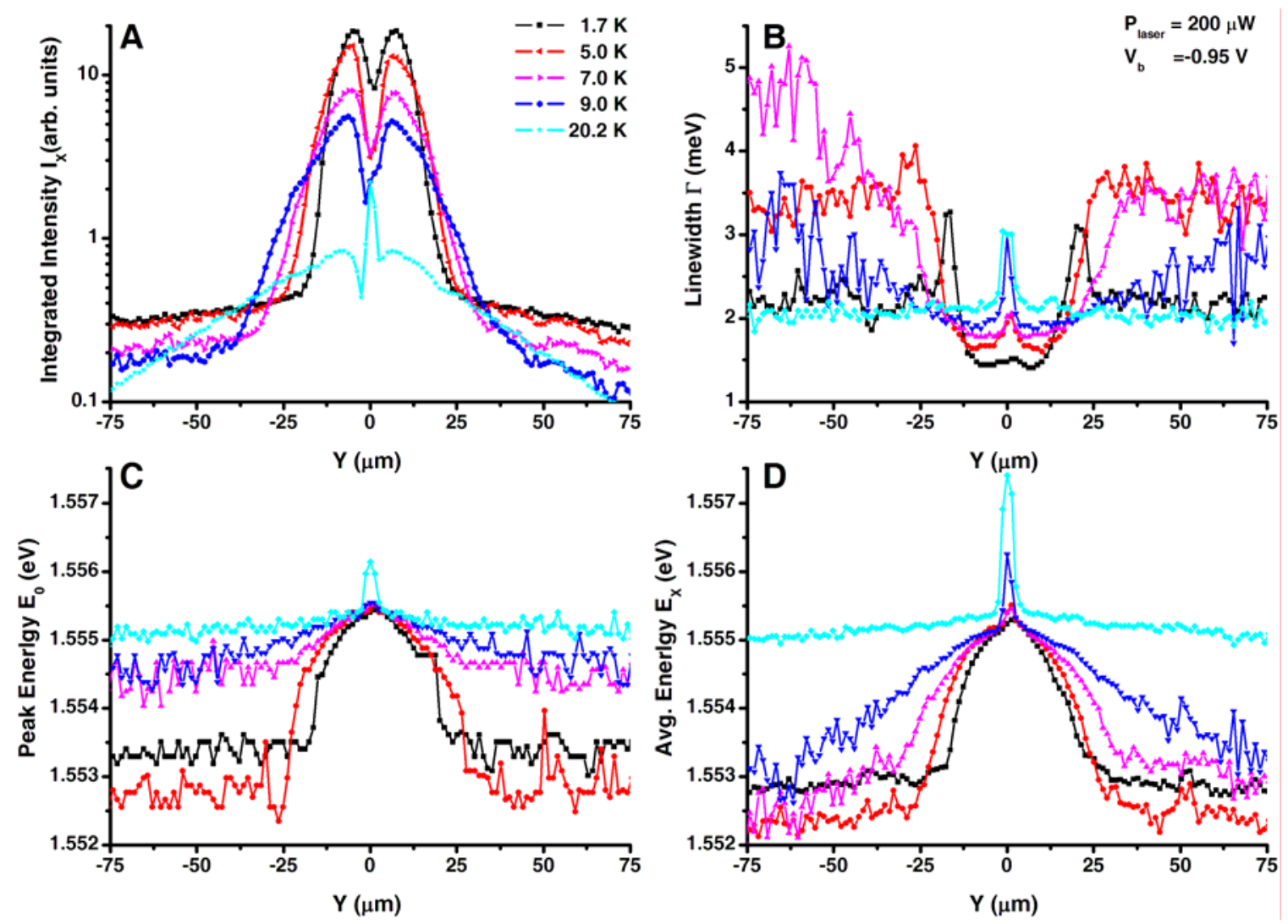

Figure 5.8 Spatially-resolved X-PL spectra versus $T$ at $P_{\text {laser }}=200 \mu \mathrm{W}$. (A) Integrated X-PL intensity $\left(\mathrm{I}_{0}\right)$. (B) X-PL linewidth (Г). (C) X-PL peak energy $\left(E_{0}\right)$. (D) X-PL average energy $\left(E_{X}\right)$. 
One of the main advantages of spatially resolved PL spectroscopy over PL imaging is that the PL emissions from Xs and $\mathrm{n}^{+}$-GaAs layers are recorded in the same measurement. Thus, the X PL can be accurately compared with the PL emissions from the $\mathrm{n}^{+}-\mathrm{GaAs}\left(\mathrm{n}^{+}-\mathrm{PL}\right)$, where carrer recombination processes are fast and well-known. Comparing indirect-X PL and $\mathrm{n}^{+}-\mathrm{PL}$ dependence on parameters such as $T, P_{\text {laser, }}$ and $V_{b}$ can thus reveal unusual physical properties of the indirect-X. In addition, the linear dependence of the $\mathrm{n}^{+}-\mathrm{PL}$ on the excitation intensity $\left(P_{\text {laser }}\right.$ or $\left.I_{\text {laser }}\right)$ is an excellent tool to evaluate the performance of the imaging or detecting system.

The system performance is confirmed by the excellent linear dependence of the integrated $\mathrm{n}^{+}-\mathrm{PL}$ on the measured $P_{\text {laser }}$ (blue line in Figure 5.9E). In contrast, the spatially integrated indirect-X PL $\left(I_{P L, S}=\int I_{X}(y) d y\right)$ grows sublinearly with $P_{\text {laser }}$ (red circles in Figure 5.9E). After confirming the linearity of the power measurement and signal detection, we then normalized the $T$ dependent $I_{P L, S}$ with respect to the $P_{\text {laser }}$ value (i.e. $\mathrm{I}_{\mathrm{PL}, \mathrm{S}}(T) / P_{\text {laser }}$ ) (Figure 5.9C). Due to the sublinear growth of $\mathrm{I}_{\mathrm{PL}, \mathrm{S}}$ with $P_{\text {laser }}$ at low temperature, $\mathrm{I}_{\mathrm{PL}, \mathrm{S}}(T) / P_{\text {laser }}$ actually decreases with increasing $P_{\text {laser }}$ in general. As the excitation density increases, $\mathrm{I}_{\mathrm{PL}, \mathrm{S}}(T)$ curves become progressively flatter, consistent with the results deduced from PL imaging (Figure 5.4C). To further characterize the $T$ dependent indirect-X PL, $\mathrm{I}_{\mathrm{PL}, \mathrm{S}}(T)$ was normalized by dividing $\mathrm{I}_{\mathrm{PL}, \mathrm{S}}(T)$ by the integrated $\mathrm{n}^{+}-\mathrm{PL}\left(\mathrm{I}_{\mathrm{n}-\mathrm{PL}}\right)$ in the same measurement. For low $P_{\text {laser }}$ values, the ratio $\mathrm{R} \equiv \mathrm{I}_{\mathrm{PL}, \mathrm{S}}(T) / \mathrm{I}_{\mathrm{n}-\mathrm{PL}}$ is almost independent of $P_{\text {laser }}$ at $T<2 \mathrm{~K}$; however, R drops faster with increasing $T$ for lower $P_{\text {laser }}$. For $P_{\text {laser }}=1660 \mu \mathrm{W}, \mathrm{R}$ is almost independent of $T$. These results suggest that $T$-dependent PL intensities of indirect-Xs are affected by processes other than redistribution of optically active Xs as a thermal Boltzmann distribution. 
The above analysis averages all indirect-Xs confined in the trap. We further single out the $T$-dependent peak X PL intensity $\left(\mathrm{I}_{0}\right)$, which better represents the most dense part of the confined $\mathrm{X}$ systems. When $T$ is lowered, $\mathrm{I}_{0}(T)$ increases more or less exponentially for $T>5 \mathrm{~K}$ (as indicted by the linear slope on the linear-log scale plot) for all $P_{\text {laser }}$ values studied, but the increasing rates are different (Figure 5.10). $\mathrm{I}_{0}(T)$ deviates slightly from an exponential growth (saturates) with decreasing $T$ near $T \approx 5 \mathrm{~K}$. Measurements with subKelvin temperature are required to confirm this saturation of X PL intensity. With decreasing $T$, an increase in the fraction of optically active Xs (i.e. free Xs with $\mathrm{K} \approx 0$ ) could be offset by a decrease in the fraction of free Xs due to the localization or freezeout. This could be the cause of such a saturation of $\mathrm{I}_{0}$ for $T<5 \mathrm{~K}$.
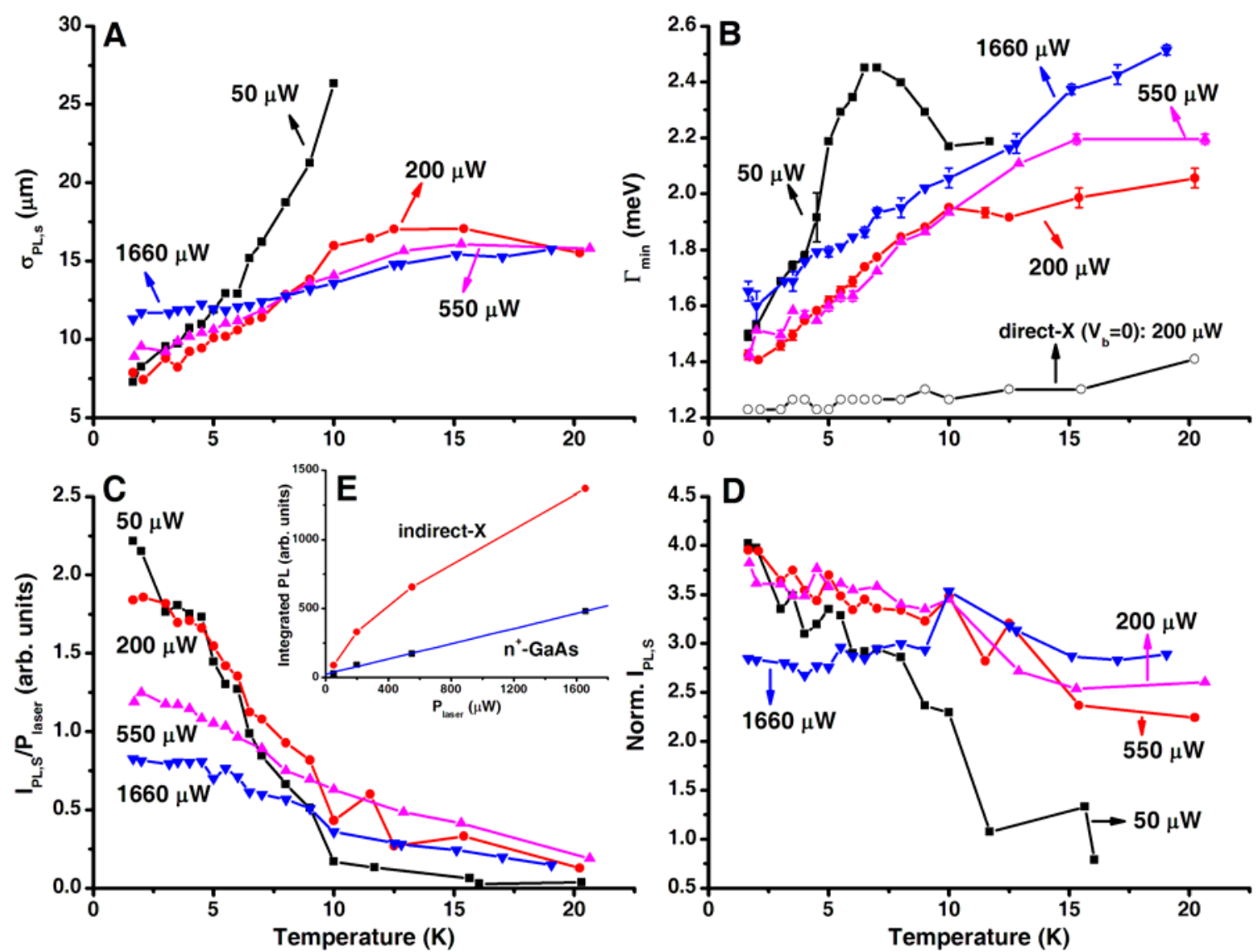

Figure 5.9 Analysis of T-dependent spatially resolved PL spectra of the confined indirect-X systems. (A) X cloud radii, $\sigma_{\mathrm{PL}, \mathrm{S}}$, calculated from 
$\mathrm{I}_{\mathrm{X}}(\mathrm{y})$. (B) Minimum indirect-X linewidths $\left(\Gamma_{\min }\right)$ and direct- $\mathrm{X}$ linewidths.

(C) Normalized spatially integrated indirect-X PL $-\mathrm{I}_{\mathrm{PL}, \mathrm{S}}(\mathrm{T}) / \mathrm{P}_{\text {laser. }}$ (D)

Normalized $T$-dependent $\mathrm{I}_{\mathrm{PL}, \mathrm{S}}-\mathrm{I}_{\mathrm{PL}, \mathrm{S}} /\left(\right.$ integrated $\left.^{+}{ }^{+}-\mathrm{PL}\right)$.
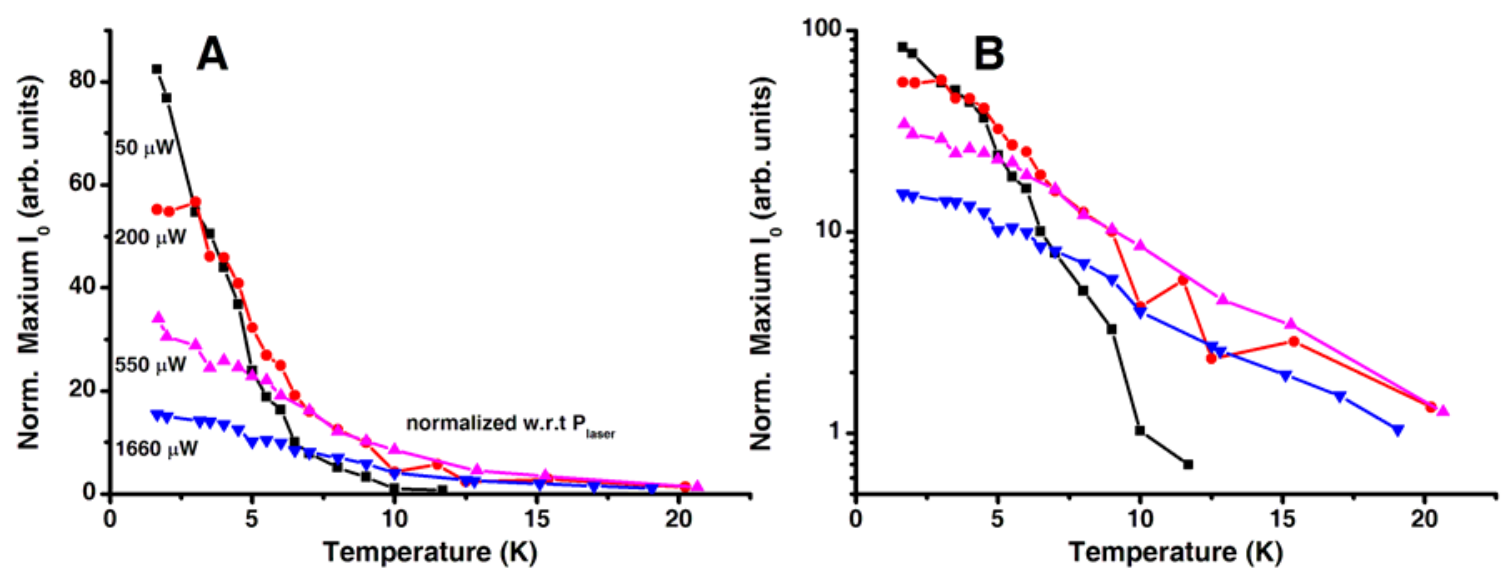

Figure 5.10 Maximum peak indirect-X PL intensity of the confined $\mathrm{X}$ system versus $T$ for $P_{\text {laser }}$ as indicated. (A) linear-linear scales. (B) linear$\log$ scales.

\subsubsection{Comparison with remote homogeneous locations}

In the pervious section, we compared T-dependent indirect-X PL peaks and $\mathrm{n}^{+}-\mathrm{PL}$ emissions in the vicinity of the trap to examine the unique properties of the confined $\mathrm{X}$ cloud. In this section, we will compare the indirect-X PL peaks at the trap center with those from remote (supposedly normal) spots.

Figure 5.11 shows typical indirect-X PL peak intensity $\left(\mathrm{I}_{0}\right)$ and energy $\left(\mathrm{E}_{0}\right)$ profiles taken at $P_{\text {laser }}=1.66 \mathrm{~mW}$ and $T=1.7 \mathrm{~K}$. We analyzed the characteristics of indirect-X PL as listed in Table 4.1 for each scanned spectrum in Y. Data points for $|\mathrm{Y}|<$ $2.5 \mu \mathrm{m}$ were then averaged to represent the 'trap' center (TC), whereas data points for 65 $\mu \mathrm{m}<|\mathrm{Y}|<75 \mu \mathrm{m}$ were averaged to represent remote spots (RS). Here we only discuss the $T$ dependence of indirect-X PL peak energy $\left(E_{0}\right)$, average energy $\left(E_{X}\right)$ and linewidth $(\Gamma)$. 


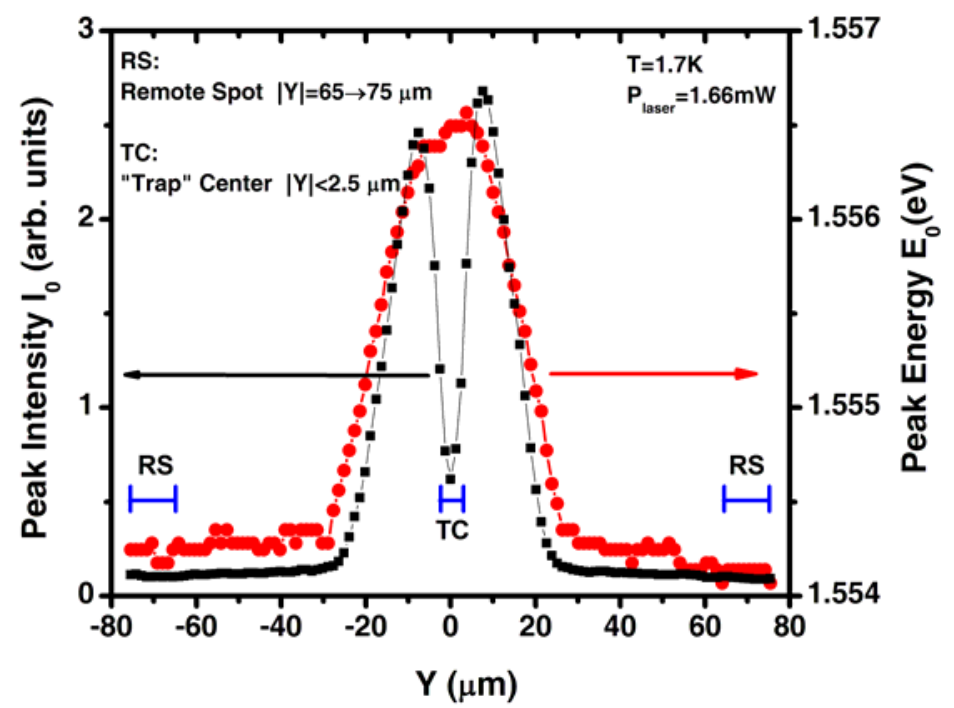

Figure 5.11 The 'trap' center (TC) versus remote spots (RS) exciton PL peak intensity $\left(\mathrm{I}_{0}\right)$ and energy $\left(\mathrm{E}_{0}\right)$ profiles taken at $P_{\text {laser }}=1.66 \mathrm{~mW}$ and $T$ $=1.7 \mathrm{~K}$.

PL or absorption of X peaks in quantum wells are expected to red shift due to the thermal bandgap shrinkage. The GaAs band gap $\left(\mathrm{E}_{\mathrm{g}}\right)$ shrinks by $\sim 1 \mathrm{meV}$ (red shift, $[148,51])$ when $T$ is increased from $2 \mathrm{~K}$ to $20 \mathrm{~K}$. This shift was determined by measuring the $T$-dependent direct-X PL (uniform HeNe excitation, bias $V_{b}=0, P_{\text {laser }}=200 \mu \mathrm{W}$ ), and was found to be less than $0.5 \mathrm{meV}$ up to $\sim 20 \mathrm{~K}$ (Figure $5.12 \mathrm{C}$ ). However, we have shown in Sec. 4.4.5 that indirect-X PL peaks can undergo energy blue shifts with increasing $T$. Though the indirect-X PL from the confined small areas ('trap') can exhibit similar Tdependent blue shift, the X PL energy shifts are more complicated.

The extent of the indirect- $\mathrm{X}$ transport is on the order of $10-20 \mu \mathrm{m}$ as seen from the spatially resolved PL spectra at a tightly focused laser spot (see for example Figure 4.5). Therefore, it is unlikely that the indirect-X PL observed at RS are emissions of the Xs generated at TC. The unusual X energy shift (red shift for T $<5 \mathrm{~K}$ and blue shift for T $>5 \mathrm{~K}$ ) and narrowing of linewidth $\Gamma$ suggest that indirect-Xs are formed at RS for $\mathrm{T}>5 \mathrm{~K}$. 

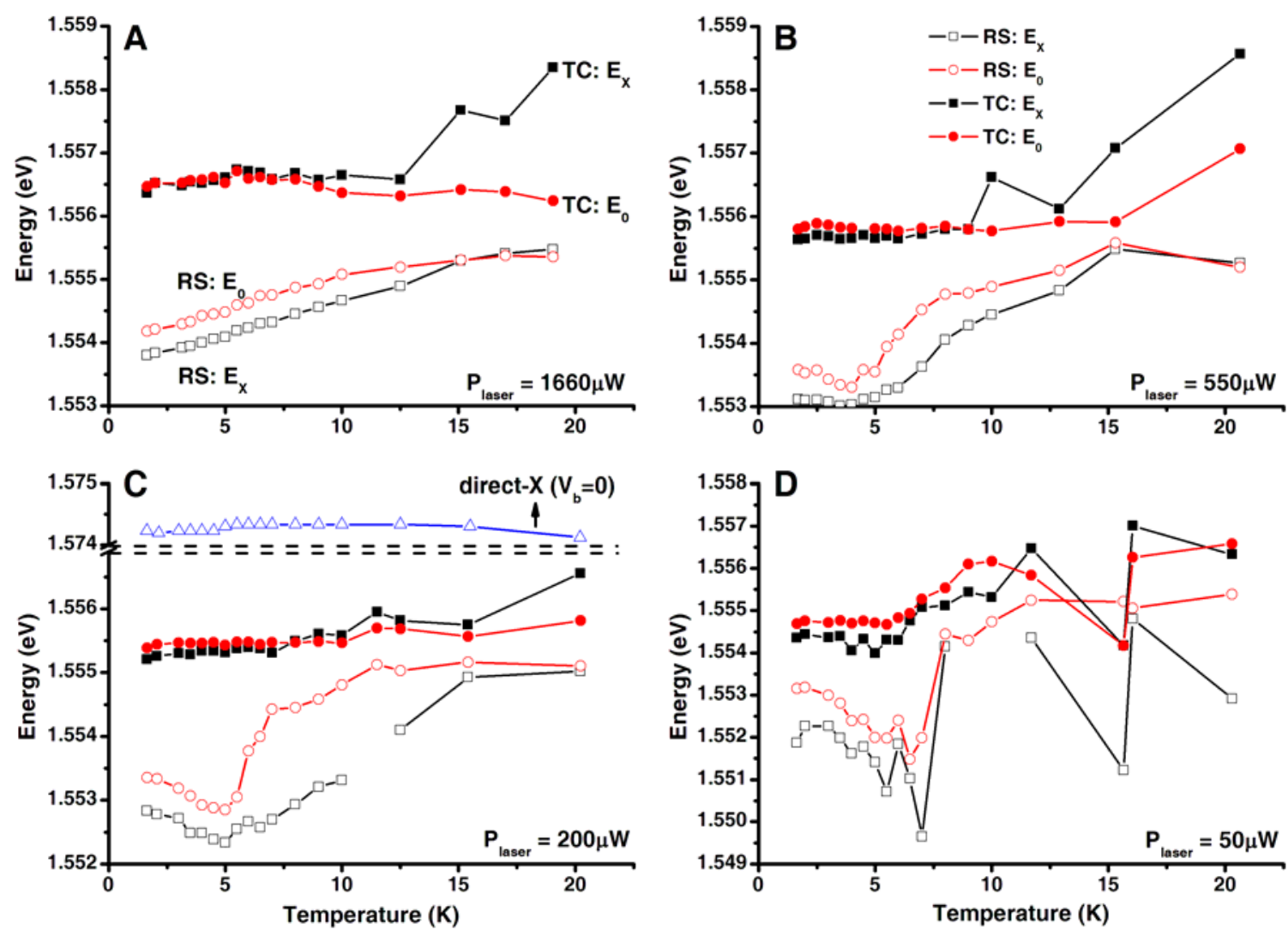

Figure 5.12 Temperature-dependent exciton peak energies $\left(\mathrm{E}_{0}\right)$ and average energies $\left(\mathrm{E}_{\mathrm{X}}\right)$ : the 'Trap' Center (TC) versus Remote Spots (RS).

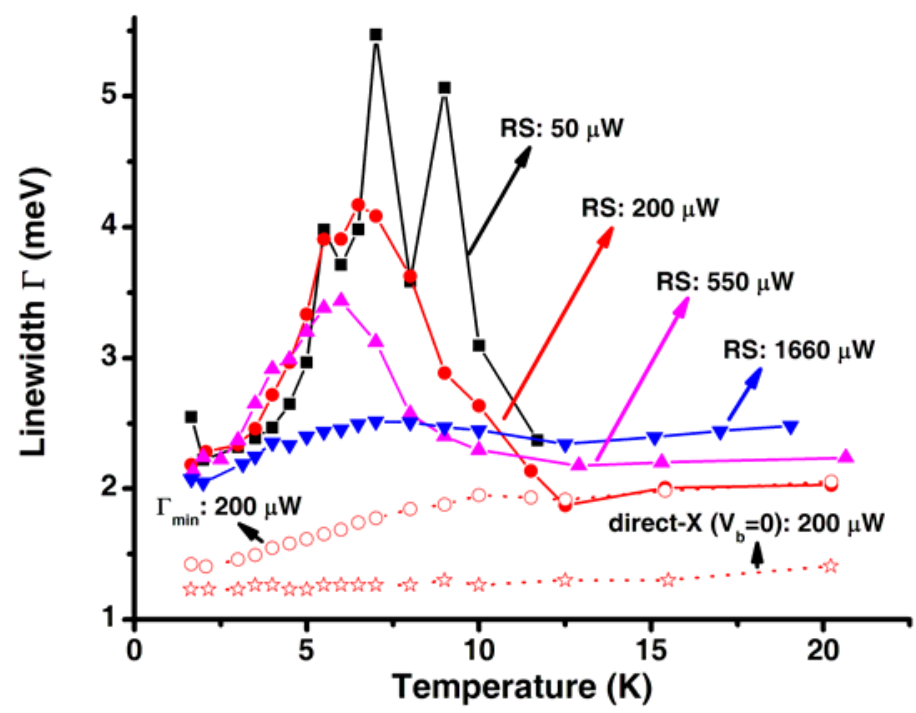

Figure 5.13 Temperature-dependent exciton PL linewidth $(\Gamma)$ at remote spots. 


\subsection{Remote local excitation}

\subsubsection{Photoluminescence imaging}

In the previous section, we described extensively the properties of an $\mathrm{X}$ cloud confined in a small area ('trap') under a uniform excitation, where photocarriers are generated almost across the whole $(780 \mu \mathrm{m})^{2}$ area of the mesa. Confined X clouds were also formed even with a local excitation hundreds of micrometers away, suggesting macroscopic transport of carriers. To further understand the collection and formation mechanisms of these confined $\mathrm{X}$ systems, we performed $\mu$-PL imaging, $\mu$-PL spectroscopy and TRPL under a remote tight-focused (spot radius $\sim 10 \mu \mathrm{m}$ or less) CW HeNe or pulsed diode laser excitation. Here indirect-X PL emissions from 'traps' can be well separated from those from the excitation spot.

The PL images in Figure 5.14 show evolution of indirect-X PL clouds versus $P_{\text {laser }}$ at $T=1.7 \mathrm{~K}$ and $V_{b}=-0.95 \mathrm{~V}$ (electric field $\sim 23 \mathrm{kV} / \mathrm{cm}$ ). A tightly focused (radius $\sim 6 \mu \mathrm{m}) \mathrm{HeNe}$ laser excited at a spot $100 \mu \mathrm{m}$ and $400 \mu \mathrm{m}$ away from Trap-A and Trap-B, respectively. At low $P_{\text {laser, }}$ a remarkable $\sim 300 \mu \mathrm{m}$ diameter modulated ring-like PL pattern appeared around Trap-B (Figure 5.15) despite the large distance between Trap-B and the excitation spot. Such symmetric PL rings cannot be attributed to pure carrier transport in the CQW plane. The clouds exhibit an annular shape with a central darker region for moderate and high $P_{\text {laser }}$ When $P_{\text {laser }}$ is increased, the annular-shaped X PL cloud contracts until a small $\sim(10 \mu \mathrm{m})^{2}$ area is reached whereupon it expands as the power is further increased. This is consistent with the phase diagram reported in Sec. 5.3.2 under a uniform excitation. 

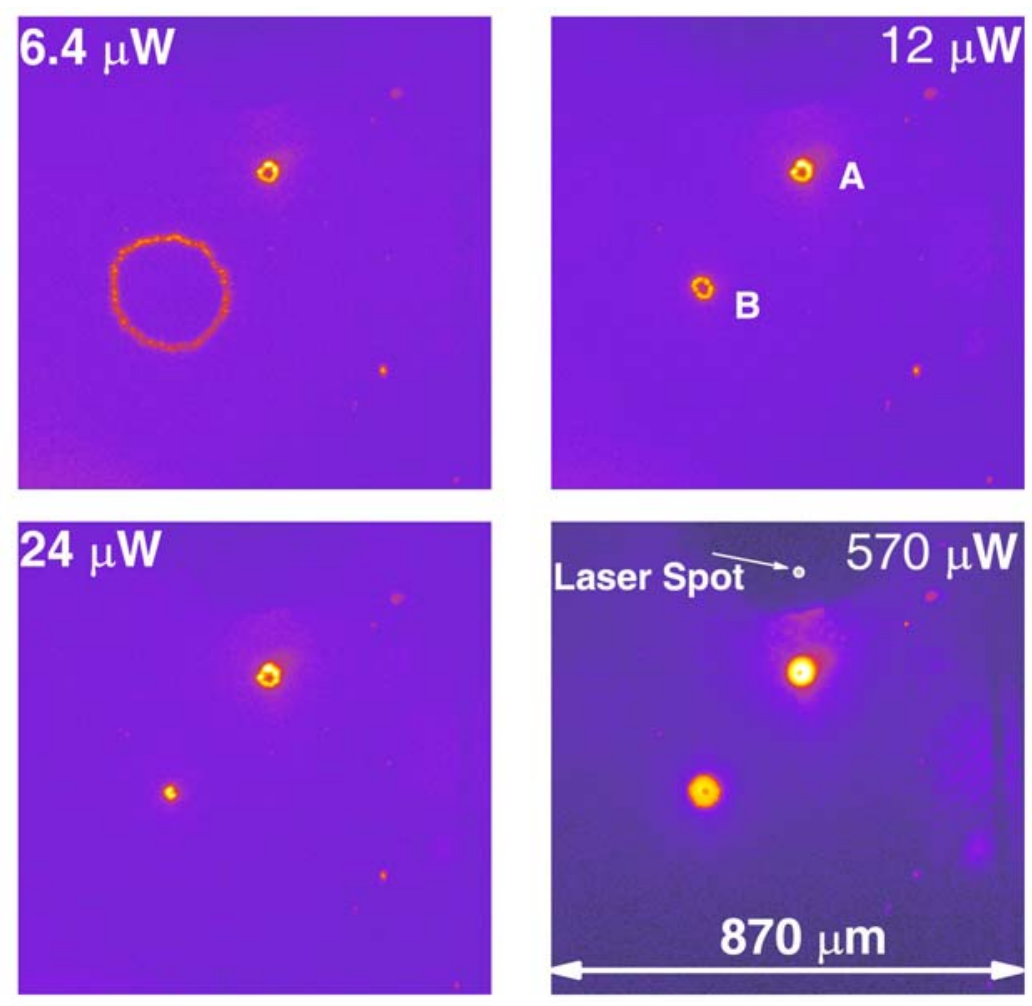

Figure 5.14 Indirect-X PL images under a remote local excitation for $P_{\text {laser }}$ as indicated. The color map is normalized and on a log scale. A $\mathrm{HeNe}$ laser of a spot radius $\sim 6 \mu \mathrm{m}$ excited $400 \mu \mathrm{m}$ away from Trap-B. The excitation spot is indicated by the white circle and was blocked when the image was taken to avoid saturating the CCD camera.

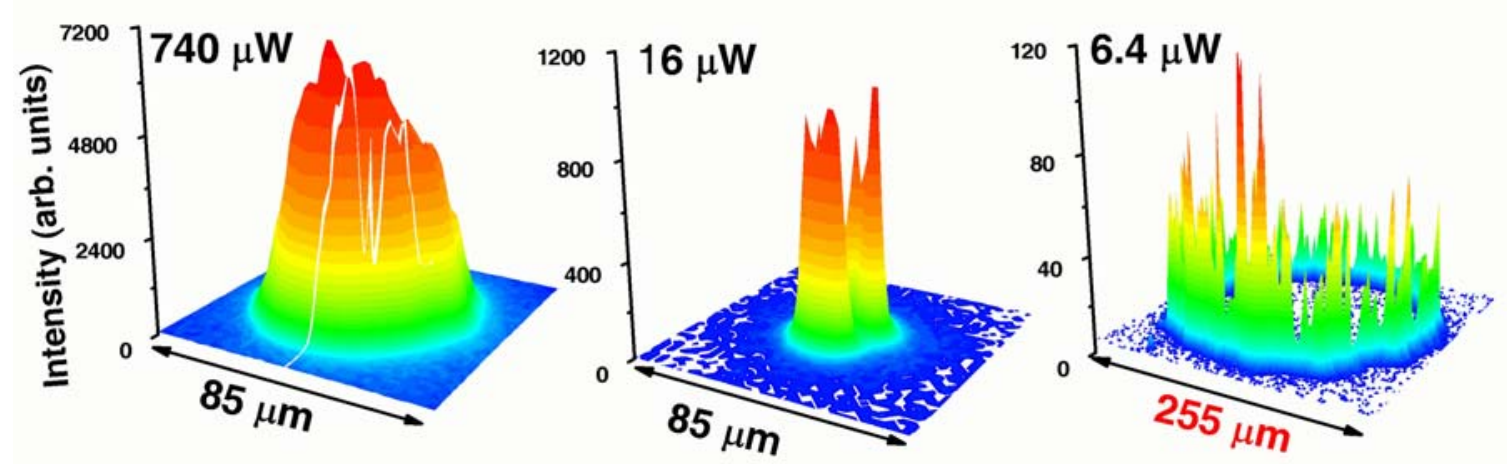

Figure 5.15 False-color 3D PL images around Trap-B. A fragmented ring PL pattern of a diameter exceeding $\sim 300 \mu \mathrm{m}$ can be observed for $P_{\text {laser }}<6$ $\mu \mathrm{W}$.

We compared $\mathrm{I}_{\mathrm{PL}}$ from the cloud under uniform and remote $(\sim 100 \mu \mathrm{m}$ away) localized HeNe excitation (Figure 5.16). Under uniform excitation, $\mathrm{I}_{\mathrm{PL}}$ varies 
approximately linearly with $P_{\text {laser }}$ at low density and sublinearly at high density; whereas under remote localized excitation (see inset X-cloud PL images), $\mathrm{I}_{\mathrm{PL}}$ first grows linearly and then exhibits a kink followed by a superlinear growth at $P_{\text {laser }} \approx 200 \mu \mathrm{W}$. These indicate that the formation of indirect-Xs whithin these confined areas are not simple collection of Xs photogenerated by laser excitation.

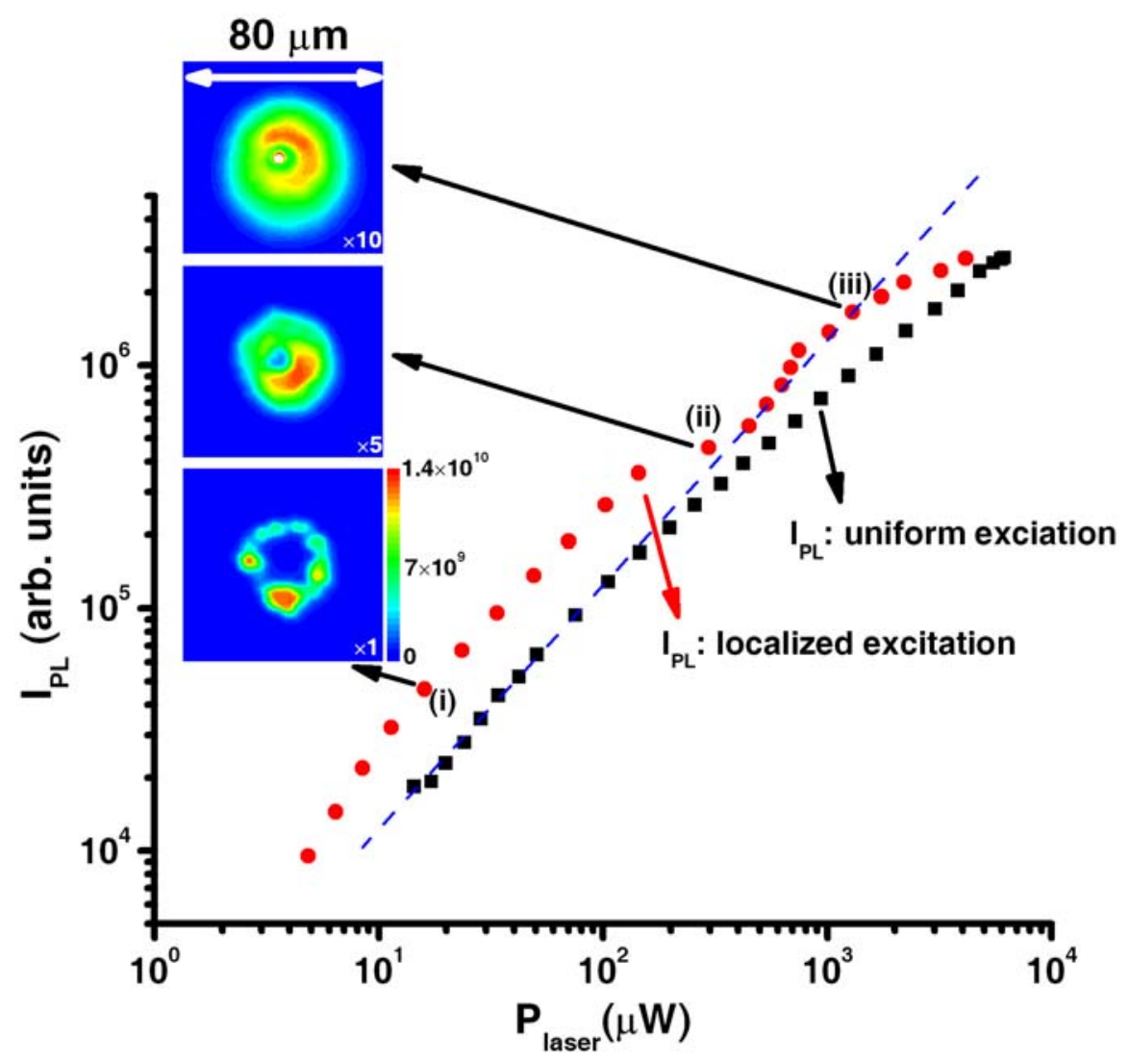

Figure 5.16 Spatially integrated indirect-X PL ( $\left.\mathrm{I}_{\mathrm{PL}}\right)$ under uniform (black squares) and remote $(\sim 100 \mu \mathrm{m}$ away) localized (red circles) $\mathrm{HeNe}$ excitation. The blue dash line is a guidance of linear dependence. The inset PL images are taken at $P_{\text {laser }}=15,290$, and $1100 \mu \mathrm{W}$. The color bar scale represents the estimated optically active $\mathrm{X}$ density for images scaled by $\times 1, \times 5$, and $\times 10$, respectively.

\subsubsection{Photoluminescence spectroscopy}

Traps are electrically active as indicated by the $V_{b}$-dependent PL spectra shown in

Figure 5.17A and B for Trap-A. The linear energy shift of well defined PL peaks seen for 
$-1 \mathrm{~V}<V_{b}<-0.5 \mathrm{~V}$ confirms the formation of well-defined indirect-Xs. This range of $V_{b}$ varies slightly under different $P_{\text {laser }}$ or for different traps. Only under these bias voltages, does the $\mathrm{X}$ cloud contract and expand as described previously. Moreover, the photocurrent depends on the distance of the excitation to the traps, implying formation of current filaments through trap sites (see Sec. 7.2.2).

\subsubsection{Time-resolved photoluminescence}

We also studied spatially- and time-resolved photoluminescence (TRPL) of these confined indirect-X systems under remote local pulsed laser excitation. The sample was excited by a pulsed laser diode with the following specifications: energy $\mathrm{h} v=1.944 \mathrm{eV}$, repetition rate $=100 \mathrm{kHz}$, pulse duration $\approx 20 \mathrm{~ns}$, and spot radius $\sigma_{\mathrm{r}} \approx 7.5 \mu \mathrm{m}$.

Figure 5.17C shows $V_{b}$-dependent indirect-X TRPL curves, which exhibit extremely long ( $\mu \mathrm{s})$ oscillatory decays. This long PL decay as compared to the $\sim 10$-ns to 100-ns indirect-X lifetime implies a continuous formation of indirect-Xs through photoassisted transport across the structure. The oscillatory PL decay also suggests the buildup of space charges (cf Sec. 7.5.2).

Figure 5.19 shows the TRPL versus $\Delta y$, the distance between the excitation and Trap-A. The long PL decay component disappears as $\Delta \mathrm{y} \leq 50 \mu \mathrm{m}$, indicating a draining of photogenerated carriers. Rise times of TRPL correspond on average to $\sim 10^{4} \mathrm{~cm} / \mathrm{s}$ carrier velocity, which deserves comment. If the indirect-Xs observed at Trap-A are due to a collection of Xs or photocarriers generated at the laser excitation spot, to reach TrapA $\sim 100 \mu \mathrm{m}$ or more away, these carriers have to travel more than $1 \mu$ s before recombinations. However, indirect-Xs and photocarriers typically have lifetimes on the order of $100 \mathrm{~ns}$ or less. Therefore, indirect-Xs observed near 'traps' are unlikely due to 
direct collection of Xs or carriers photogenerated at the laser excitation spot. Photoinduced injection of both electrons and holes near these localities seems to be a more plausible scenario.
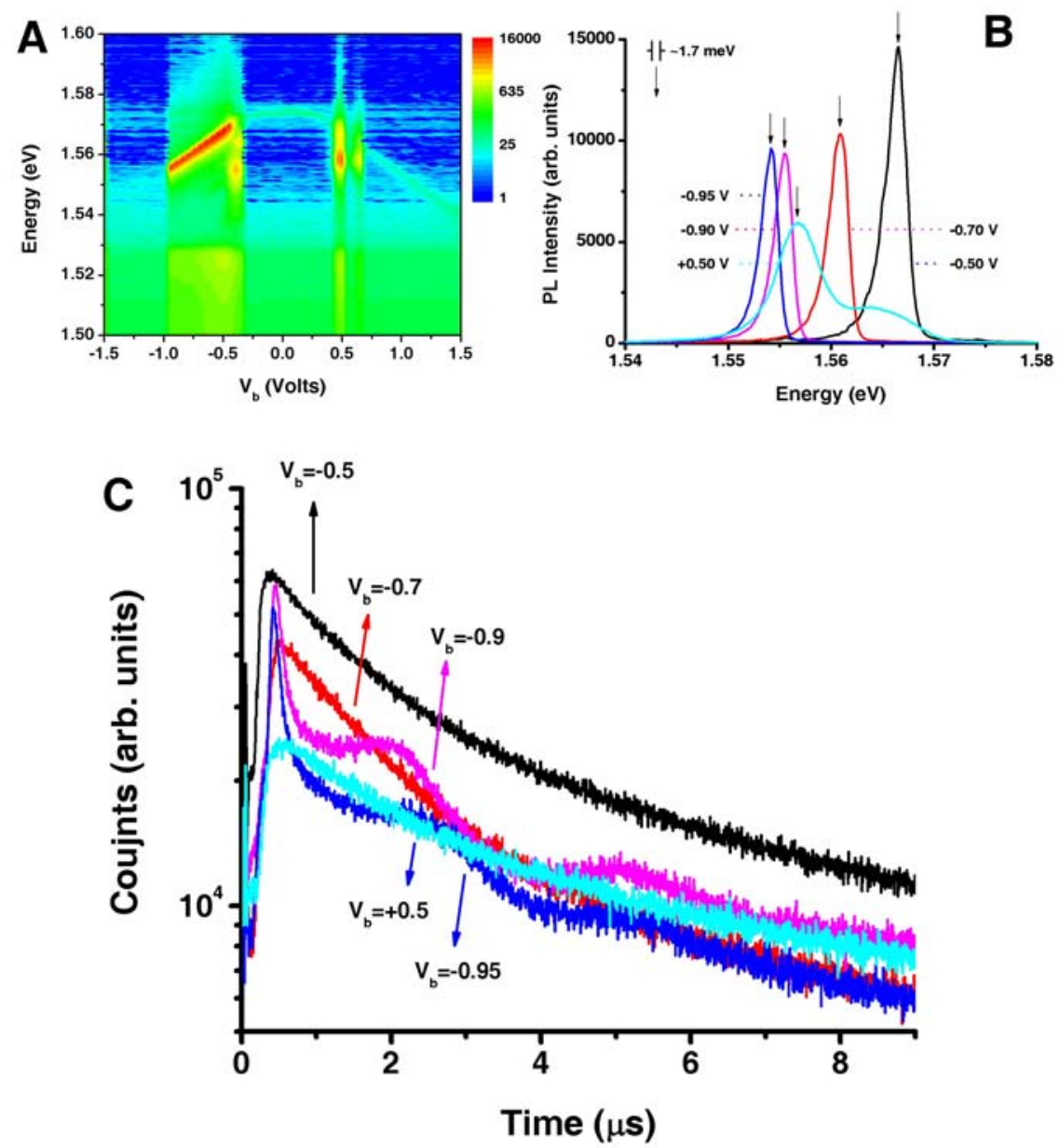

Figure 5.17 (A) Two-dimensional color map of the $V_{b}$-dependent PL spectra measured for Trap-A. (B) Selected PL spectra for $V_{b}$ as indicated. The arrows indicate the central wavelengths of the indirect-X PL detected by a micro-channel plate photomultiplier behind the exit slit (bandwidth $\sim 1.7 \mathrm{meV}$ ) of the spectrometer. (C) $V_{b}$-dependent indirect-X TRPL for X cloud near Trap-A. (Laser: $\Delta \mathrm{y} \approx 150 \mu \mathrm{m}$, pulse duration $=20 \mathrm{~ns}$, repetition rate $=100 \mathrm{kHz}$ and $P_{\text {laser }} \approx 24 \mu \mathrm{W}$ ).

The indirect-X TRPL was also measured as a function of average laser excitation power $\left(P_{\text {laser }}\right)($ Figure 5.19) and temperature $(T)$ (Figure 5.20). They both exhibited a nonexponential PL decay, which indicated that indirect-Xs within these confined small areas are the result of formation through complicate processes rather than simple 
collection of preexisting photogenerated indirect-Xs. The TRPL described above were taken by spatially averaging all confined indirect-Xs near traps. The chaotic formation and radiative recombination of indirect-Xs in these confined areas are seen more clearly from the spatially resolved TRPL (Figure 5.21).

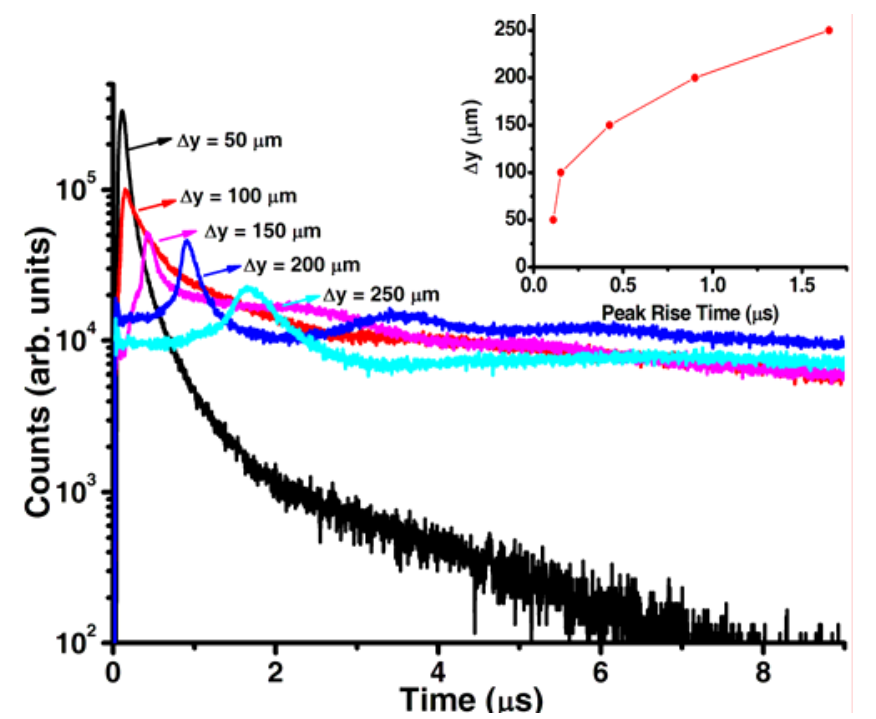

Figure 5.18 TRPL of indirect-Xs from Trap-A versus $\Delta \mathrm{y}$ at $V_{b}=-0.95 \mathrm{~V}$ and $T=1.7 \mathrm{~K}$. The inset shows $\Delta \mathrm{y}$-dependent $\mathrm{PL}$ rise times, corresponding on average to a $\sim 10^{4} \mathrm{~cm} / \mathrm{s}$ carrier velocity. (Laser: pulse duration $=20 \mathrm{~ns}$, repetition rate $=100 \mathrm{kHz}$ and $P_{\text {laser }} \approx 24 \mu \mathrm{W}$ ).

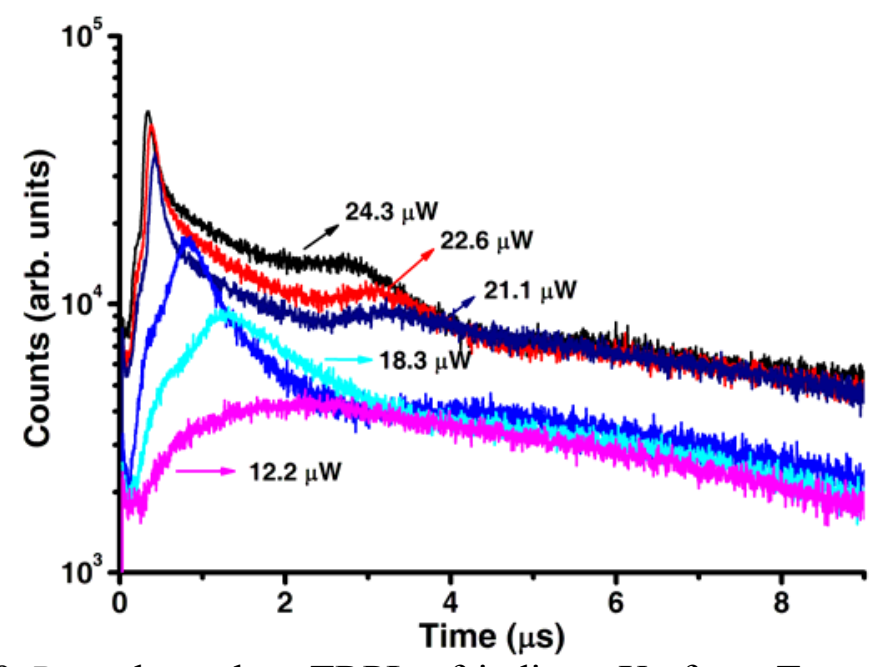

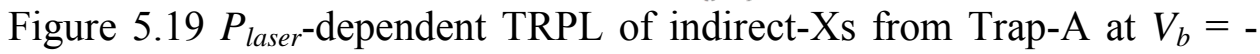
$0.95 \mathrm{~V}$. (Laser: $\Delta \mathrm{y} \approx 150 \mu \mathrm{m}$, pulse duration $=20 \mathrm{~ns}$, and repetition rate $=$ $100 \mathrm{kHz})$. 

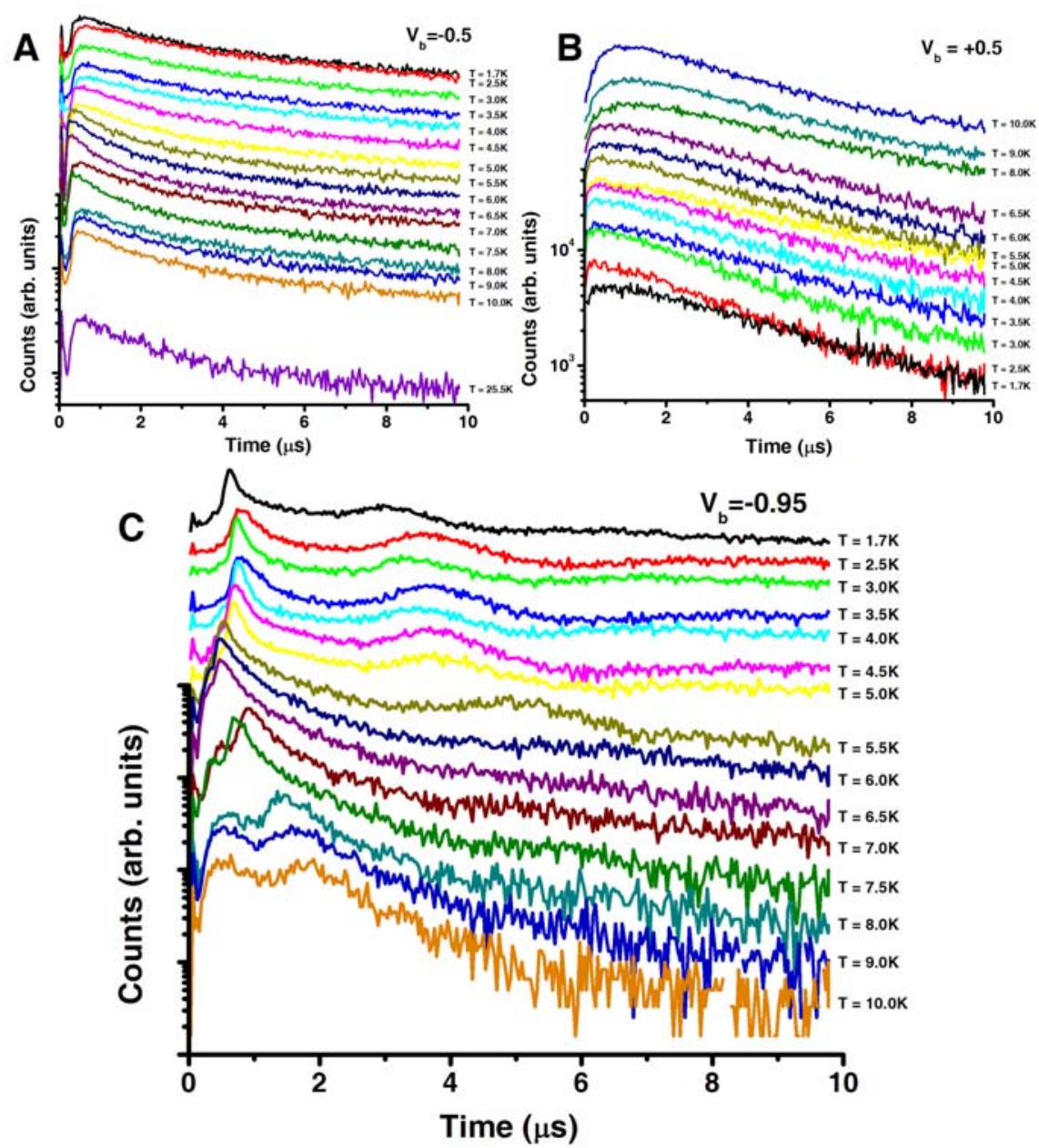

Figure 5.20 T-dependent TRPL of indirect-Xs from Trap-A at $V_{b}=-0.5$, +0.5 and $-0.95 \mathrm{~V}$. (Laser: $\Delta \mathrm{y} \approx 150 \mu \mathrm{m}$, pulse duration $=20 \mathrm{~ns}$, repetition rate $=100 \mathrm{kHz}$, and $P_{\text {laser }} \approx 24 \mu \mathrm{W}$ ).

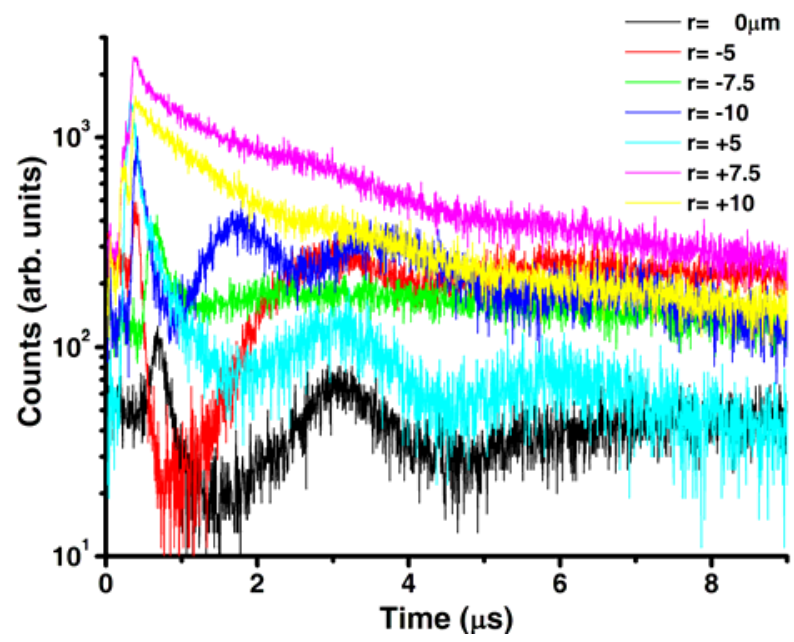

Figure 5.21 Spatially-resolved TRPL of indirect-Xs from Trap-A at $V_{b}=-$ $0.95 \mathrm{~V}$ at $\mathrm{T}=1.7 \mathrm{~K} . \mathrm{r}$ is the distance from Trap-A center. (Laser: $\Delta \mathrm{y} \approx 150$ $\mu \mathrm{m}$, pulse duration $=20 \mathrm{~ns}$, repetition rate $=100 \mathrm{kHz}$, and $P_{\text {laser }} \approx 24 \mu \mathrm{W}$ ). 


\subsection{Conclusion}

In this chapter, we have described the formation and phase diagram of degenerate $\mathrm{X}$ systems in quasi-2D confined small areas ('traps'). For the phase diagram, the thermodynamic quantities are the spatial and energy distributions (including emission energies and linewidths) of the optically active indirect Xs, measured with diffraction limited resolution by PL as a function of temperature $(T)$ and laser excitation $\left(P_{\text {laser }}\right)$. The phase diagram displays distinct regimes with one, in particular, that corresponds to the

concentration of tens of thousands Xs in small areas $(\sim 10 \mu \mathrm{m})^{2}$, at low temperature and moderate excitation. Further investigations are required to search for collective quantum effects in these cold X systems. Spatially- and time-resolved PL (TRPL) spectroscopy were used to study the formation mechanisms of these X systems. TRPL exhibited extremely long ( $\mu \mathrm{s})$ oscillatory decays. These results imply that the indirect-Xs clouds are not collection of preexisting photogenerated Xs or carriers, but are formed in the immediate vicinity of traps through photoassisted carrier capture and injection across the $n+-i-n+$ structure, and a buildup of space charges. These formation mechanisms will be detailed further in Sec. 7.3. 


\section{Chapter 6 Other phenomena}

\subsection{Introduction}

We have conducted measurements by exploring various parameters to study the properties of the coupled quantum well (CQW) samples. Table 6.1 lists the measurements and parameters of the experimental systems. Exploring the phase space with various parameters, we have found that the CQW studied exhibited many unusual phenomena. Some unusual properties of the CQW sample in general have been described in Chapter 4. Properties and extensive phase diagrams of the confined excitons were also in Chapter 5. There are many other interesting physical phenomena observed in the system, which will not be covered in detail in this dissertation. For completeness, we will briefly review some other interesting physical phenomena in the studied CQW sample.

Table 6.1 List of measurements and experimental parameters.

\begin{tabular}{|l|l|l|}
\hline \multirow{2}{*}{ Measurements } & \multicolumn{2}{|c|}{ Parameters/Variables } \\
\hline \multirow{4}{*}{ SL spectroscopy } & $T$ & Temperature \\
\cline { 2 - 3 } Time resolved PL (TRPL) spectroscopy & $P_{\text {laser }}$ & Average laser power \\
\cline { 2 - 3 } & $V_{b}(F)$ & Bias voltages (electric fields) \\
\cline { 2 - 3 } PL imaging (or PL tomography) & $B$ & Magnetic fields \\
\cline { 2 - 3 } & $t$ & Time \\
\cline { 2 - 3 } Photocurrent (steady and transient photocurrent) & $\begin{array}{l}\text { CW excitation \& } \\
\text { Pulsed excitation }\end{array}$ & $\begin{array}{l}\text { (i) Energy (hv) } \\
\text { (ii) Spot size (uniform or local) }\end{array}$ \\
\cline { 2 - 3 } & $\begin{array}{l}\text { Pulsed excitation } \\
\text { only }\end{array}$ & $\begin{array}{l}\text { (i) Pulse amplitude } \\
\text { (ii) Pulse width/duration } \\
\text { (iii) Repetition rate }\end{array}$ \\
\hline
\end{tabular}




\subsection{Ring-pattern photoluminescence}

\subsection{1 $\mu$-PL imaging and $\mu$-PL spectroscopy}

Under local HeNe laser excitation, PL can be observed up to more than $300 \mu \mathrm{m}$ away in the form of a fragmented ring (necklace-like) (Figure 6.1). Between the PL ring and the excitation spot, there is an extended dark annular region, whose size grows with increasing $P_{\text {laser }}$ but decreases with increasing $\left|V_{b}\right|$. PL rings were observed on various mesas and different samples processed from the same wafer. However, they appeared only for a certain range of $P_{\text {laser }}$ and $V_{b}$, typically near the negative differential region (NDR) shown in photocurrent-voltage characteristics (see Sec. 7.2.2).

Note that the PL images and spectra shown in this section (Sec. 6.2) were taken on a mesa having an open area $\sim(500 \mu \mathrm{m})^{2}$ but on a different diced sample from that was used previously. The measurements were performed in a magnetic cryostat with a microscope objective installed. The system spatial resolution was $\sim 4 \mu \mathrm{m}$.

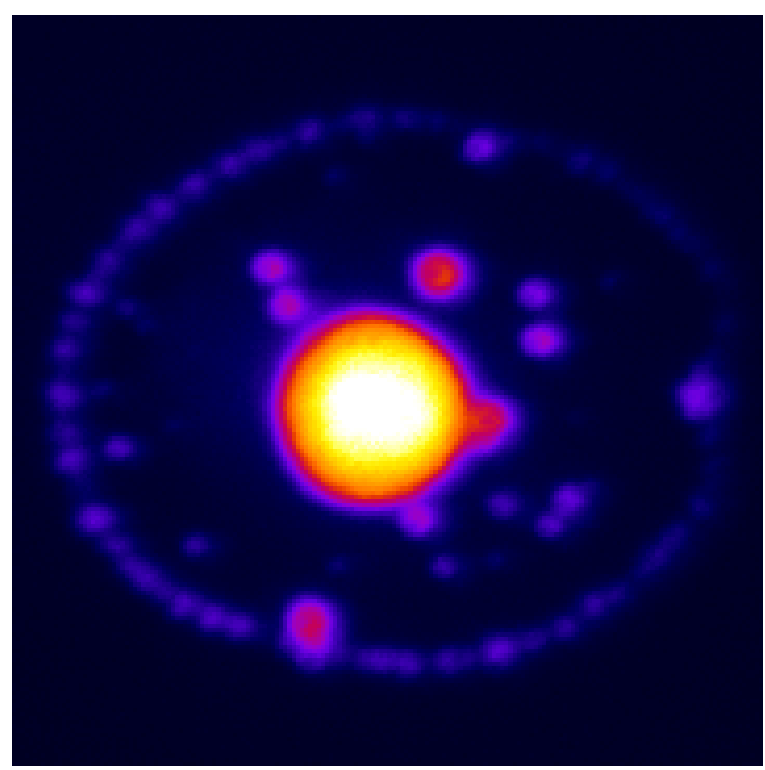

Figure 6.1 A fragmented PL ring pattern shown by a PL image measured at $T=1.7 \mathrm{~K}, P_{\text {laser }}=390 \mu \mathrm{W}$, and $V_{b}=-1.20 \mathrm{~V}$. A $10-\mathrm{nm}$ bandpass filter was used to select the indirect-X PL emissions. The central bright zone of the CCD image was saturated due to the strong PL emissions including the 
leakage PL from the $\mathrm{n}^{+}$-GaAs at the excitation spot. (View area $\sim 240$ $\mu \mathrm{m} \times 240 \mu \mathrm{m})$

To better understand the energy distribution of the indirect-Xs, we measured spatially-resolved PL spectra as a function of $P_{\text {laser }}$ by scanning a pinhole in the first confocal image plane. Figure 6.2 shows the PL spectra as a function of the distance from the excitation center $(\mathrm{Y})$ at $V_{b}=-1.22 \mathrm{~V}, P_{\text {laser }}=390 \mu \mathrm{W}$ and $T=1.8 \mathrm{~K}$.

At low $P_{\text {laser }}$, indirect-X PL distribution follows approximately the laser excitation (Figure 6.3A); whereas at high $P_{\text {laser, }}$, distant ring-pattern PL emerges at increasing radii with increasing $P_{\text {laser }}$. The spatial distribution of the indirect-X PL intensity exhibits an annular-shaped profile with a dip at the excitation center; however, the $\mathrm{X}$ energy decreased monotonically (Figure 6.3A and B). This indicates that the Xs at the excitation center are not optically active, which is likely due to heating of hot photogenerated carriers. The external fragmented PL ring patterns exhibit high contrast only for $T$ less than about $5 \mathrm{~K}[46,181]$. The occurrence of these distant PL ring patterns depends on many parameters such as the laser excitation energy $(\mathrm{h} v)$, intensity $\left(P_{\text {laser }}\right)$, spot size, pulsed or $\mathrm{CW}$ mode, bias voltage $\left(V_{b}\right)$ and magnetic field $(B)$. For some conditions, a double-ring PL pattern can appear and the 'dark' region is not completely dark. Detailed study of these PL rings is beyond the scope of this dissertation. 


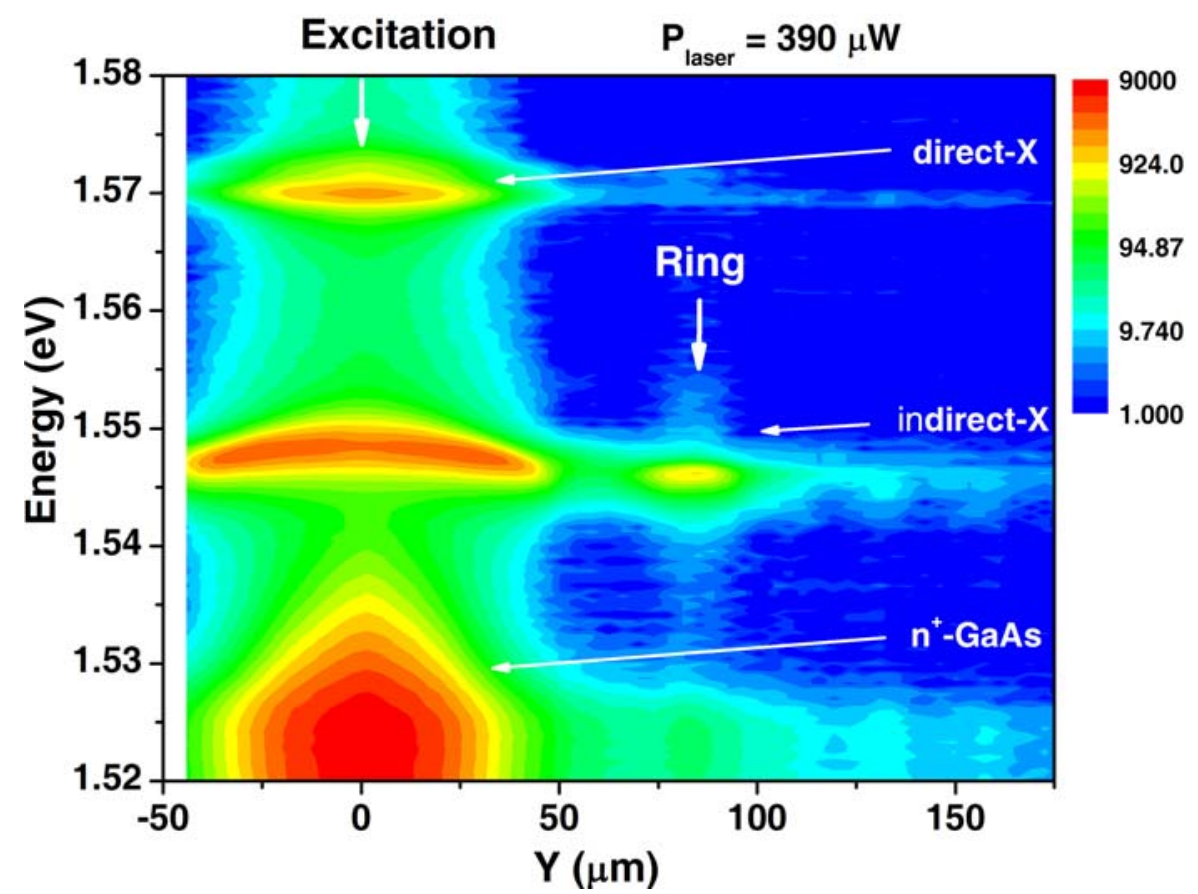

Figure 6.2 Spatially resolved PL spectra at $V_{b}=-1.22 \mathrm{~V}, P_{\text {laser }} \approx 390 \mu \mathrm{W}$ $(\mathrm{HeNe}$ ) and $T=1.8 \mathrm{~K}$. The color map is in log scale. PL lines $\sim 1.57 \mathrm{eV}$ are the direct-Xs, while the broad emissions below $\sim 1.535 \mathrm{eV}$ are from the $\mathrm{n}^{+}$-GaAs layers. PL lines between about $1.548 \mathrm{eV}$ to $1.545 \mathrm{eV}$ are indirectXs. Indirect-X PL reappeared at more than $75 \mu \mathrm{m}$ away from the laser excitation center. Data were taken on a mesa of an area $\sim(500 \mu \mathrm{m})^{2}$.
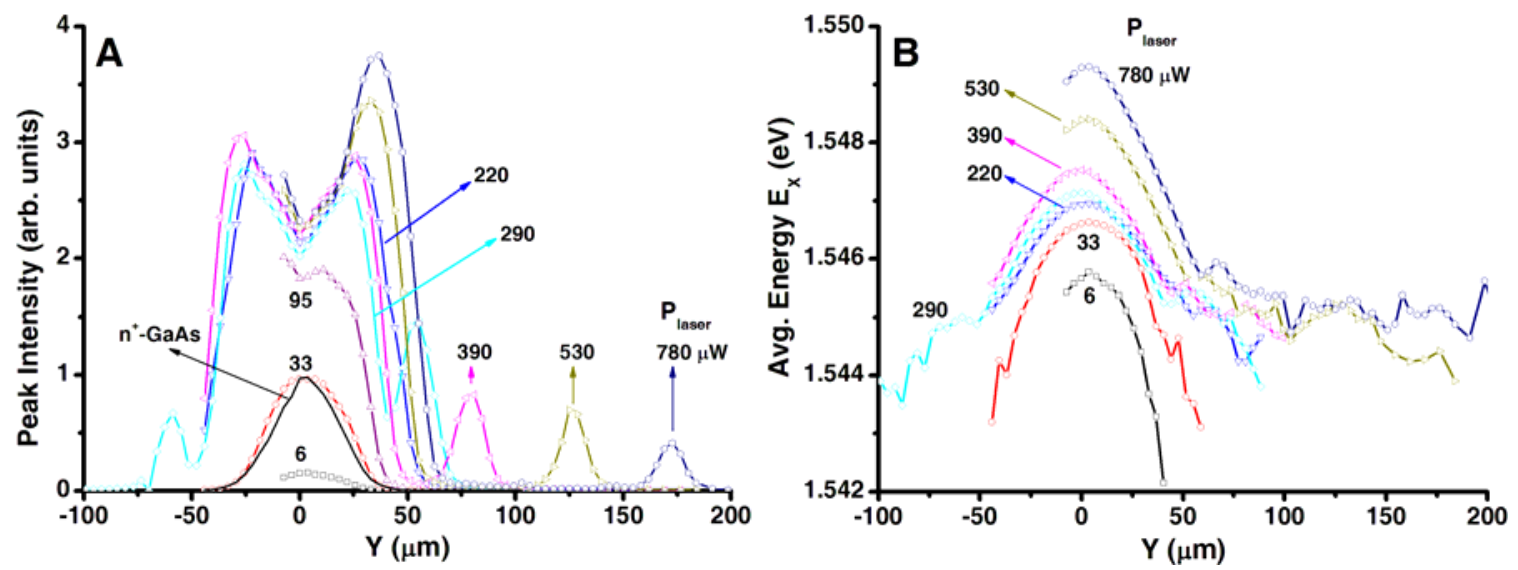

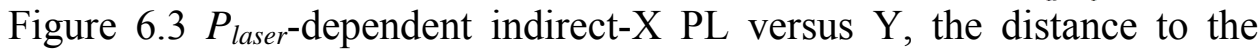
center of the laser excitation. (A) Peak intensity $\left(\mathrm{I}_{0}\right)$. (B) Average energy $\left(\mathrm{E}_{\mathrm{X}}\right)$. Experimental parameters are $V_{b}=-1.22 \mathrm{~V}, T=1.8 \mathrm{~K}$, and $P_{\text {laser }}=6$, $33,95,220,290,390,530$, and $780 \mu \mathrm{W}$, respectively. Black line in (A) is the intensity profile of the laser excitation spot determined from the $\mathrm{n}^{+}$GaAs PL emissions. The laser spot radius $\sigma_{\mathrm{r}} \approx 21 \mu \mathrm{m}$. 


\subsubsection{Time-resolved photoluminescence}

To understand the formation mechanism of these PL ring patterns, we measured time-resolved PL of the indirect-Xs from these distant ring patterns. The measurements shown below were conducted on a mesa of an area $\sim(800 \mu \mathrm{m})^{2}$ at $V_{b}=-1.13 \mathrm{~V}$ and $T=$ 1.7 K using the Janis cryostat (see Sec. 3.3.1 setup). The indirect-X PL peak intensity profile deduced from the spatially resolved time-integrated PL spectra is shown in Figure 6.4A. The 'dark' region is not as clear as that seen in Figure 6.3. This may be due to a different excitation (lower energy, pulsed, and a tight-focused spot [radius $\sigma_{\mathrm{r}} \approx 7.5 \mu \mathrm{m}$ as compared to $\sigma_{\mathrm{r}} \approx 21 \mu \mathrm{m}$ used for Figure 6.3] ). TRPL curves were measured at the rings $(\mathrm{Y}= \pm 60 \mu \mathrm{m})$ and excitation $(\mathrm{Y}=-10 \mu \mathrm{m})$, where $\mathrm{Y}$ is the distance from the laser excitation center. Indirect-X PL exhibits a fast decay $\sim 50 \mathrm{~ns}$ followed by a rise of about 300-500 ns after switch-off of the pulsed excitation (Figure 6.4B). The photocurrent transient shows a similar rise of photocurrent (see Sec. 7.2.3 below). In addition, we have mentioned previously that ring-pattern PL emissions are observed for a $V_{b}$ near the appearance of NDR in the I-V curve at the same time. These suggest that formation of indirect-X near PL rings is related to cross-well carrier transport. However, further experiments are necessary to establish a clear correlation between the PL rings (around the excitation spot, traps, and local spots) and the cross-well carrier transport induced by photoexcitation.

Finally, we note that some models based on the carrier imbalance mechanism and carrier diffusion in the QW plane were proposed $[182,181]$ to explain the formation of ring-pattern PL emissions. Based on the TRPL measurements, the in-plane carrier transport alone is unlikely to account for the formation of these PL rings. 

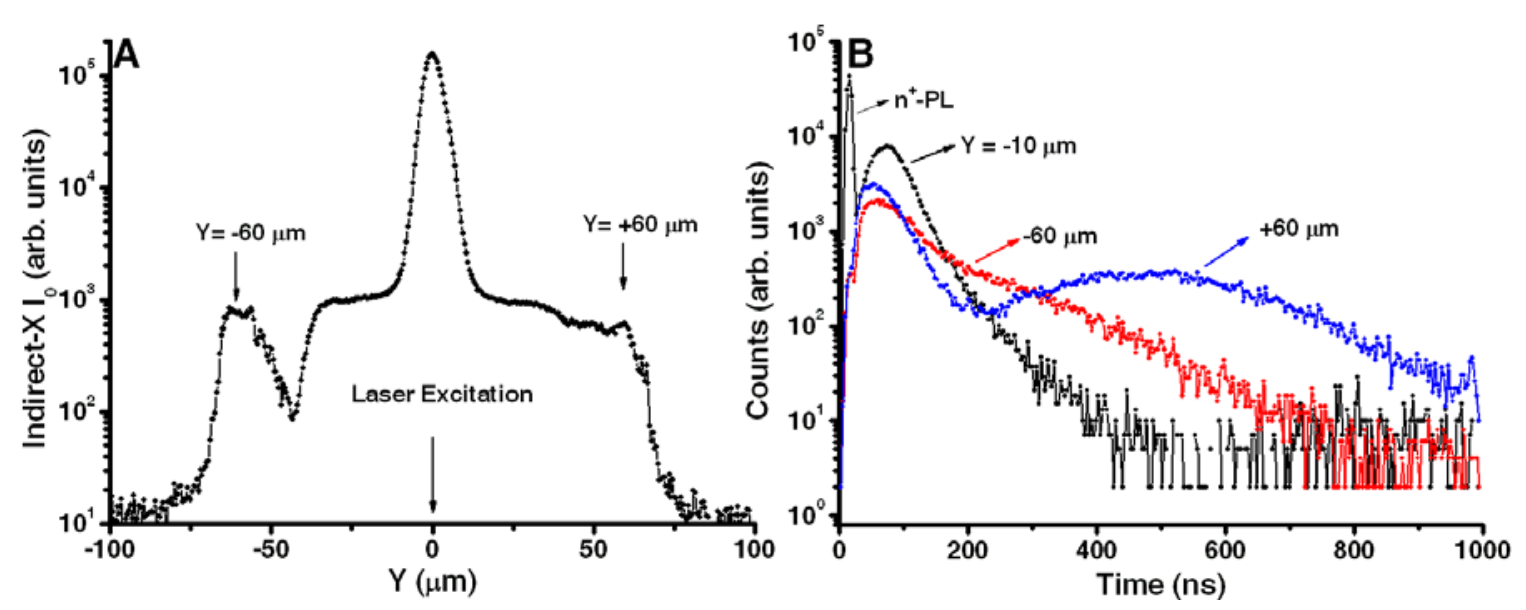

Figure 6.4 (A) Indirect-X PL peak intensity profile under a pulsed diode laser excitation. (B) Time-resolved PL of indirect-Xs: Excitation $(\mathrm{Y}=-10 \mu \mathrm{m})$ versus $\mathrm{PL}$ rings $(\mathrm{Y}= \pm 60 \mu \mathrm{m})$. (Pulsed laser excitation: $\mathrm{h} v=1.844 \mathrm{eV}$, pulse duration $=$ $20 \mathrm{~ns}$, repetition rate $=1 \mathrm{MHz}$ and average $\left.P_{\text {laser }} \approx 186 \mu \mathrm{W}\right)$.

\subsection{Local photoluminescence centers}

In Chapter 5, we have described extensively the indirect-X systems formed in confined small areas. These confined $\mathrm{X}$ cloud were observed only for certain $V_{b}$ 's as we have discussed (See Sec. 5.4.3 and Figure 5.17). Most of the results reported in Chapter 5 were obtained for $V_{b}=-0.95 \mathrm{~V}$, corresponding to $\sim 23 \mathrm{kV} / \mathrm{cm}$ electric field across the CQW active region. When the negative $V_{b}$ was decreased further to less than about -1.0 $\mathrm{V}$, many local PL centers began to emerge as shown in Figure 6.5. The PL images shown here were taken with a long-pass filter allowing high transmission for photons of wavelengths $\lambda>715 \mathrm{~nm}$. This allowed equal transmission of PL emissions from the indirect-Xs and $\mathrm{n}^{+}$-GaAs layers for any $V_{b}$. These local PL centers exhibit some characteristics similar to those strongly confined X systems ('traps'). The formation and collection of indirect-Xs near 'traps' and local PL centers are likely caused by similar mechanisms, which we will address in detail in Chapter 7. However, we will not detail the properties of local PL centers. 


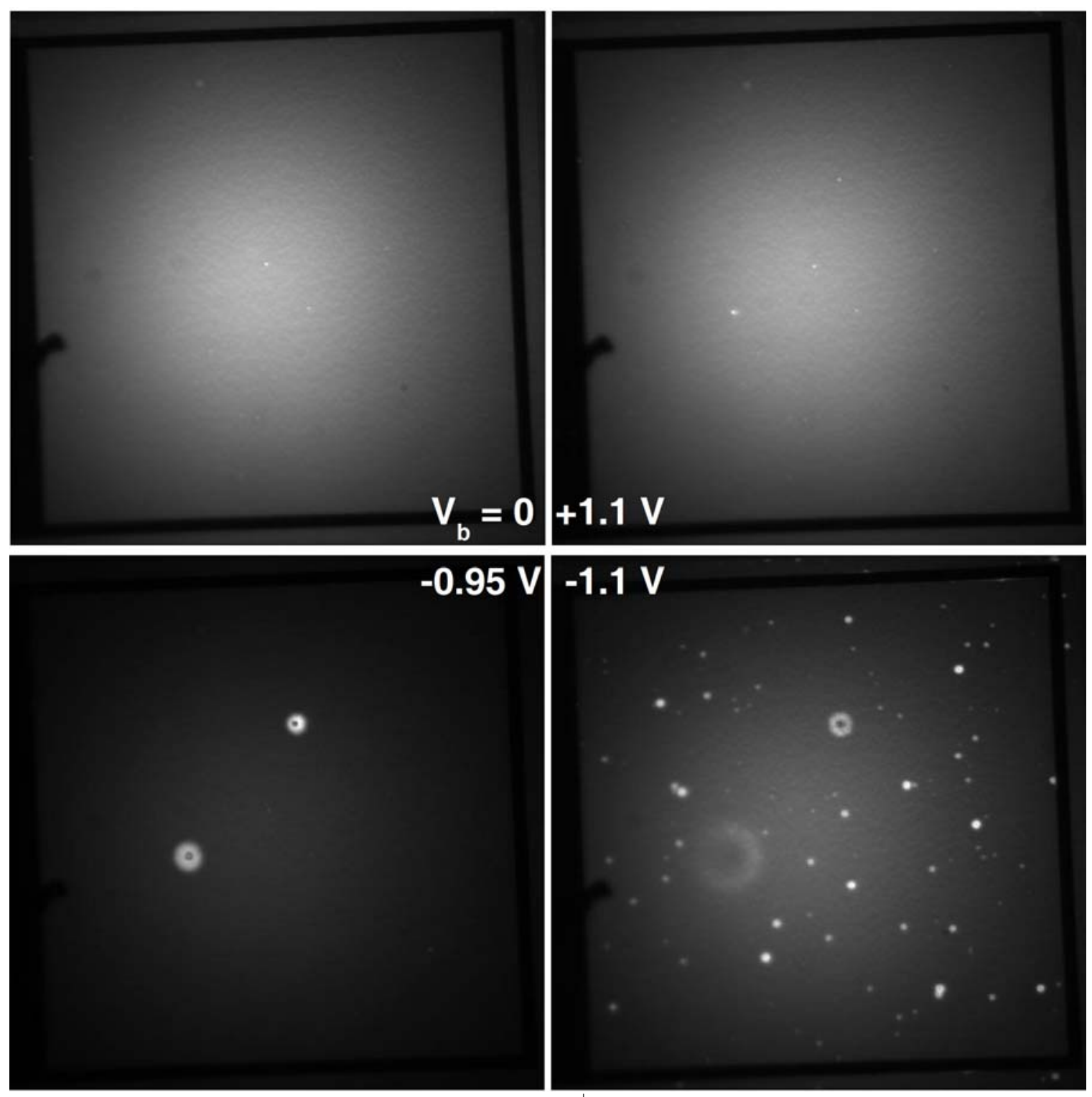

Figure $6.5 V_{b}$-dependent PL (Xs and $\mathrm{n}^{+}$-GaAs) images at $T=1.7 \mathrm{~K}$ and $P_{\text {laser }}=6440 \mu \mathrm{W}$ under a uniform HeNe excitation. Traps and local PL centers appeart only for certain range of $V_{b}$, where photocurrent is usually significant. (View area: $870 \mu \mathrm{m} \times 870 \mu \mathrm{m}$ ) 


\section{Chapter 7 Formation mechanisms}

\subsection{Introduction}

For localized excitation, photoluminescence (PL) was observed not only from the laser spot, but also far from it (i.e., from PL rings, local PL centers and 'traps'). We refer to these unusual PL emissions as "distant PL" (DPL) for brevity. DPL emissions have narrow peaks (linewidth $\Gamma \approx 2 \mathrm{meV}$ or less) and shift with applied bias voltage $\left(V_{b}\right)$, which confirm that they are radiative annihilation of spatially indirect excitons (indirect$\mathrm{Xs})$.

In this chapter, we will summarize the electrical properties of the CQW sample and compare them with the electric field dependent PL spectra. These results suggest that indirect-Xs giving rise to DPL emissions are formed through photo-induced carrier injection, trapping, transport and space charge buildup. We will also summarize the formation mechanisms of DPL and possible modeling.

\subsection{General photocurrent characteristics}

\subsubsection{Introduction}

The system under study is coupled quantum wells (CQW) in an electric field perpendicular to the layers. The undoped GaAs CQW together with AlGaAs barrier layers are embedded in an $n^{+}-i-n^{+}$structure. The electric field across the intrinsic region is tuned by varying $V_{b}$. Ideally, the quantum wells are depleted in the dark, and the carrier density and distribution can be controlled by optical excitation at a suitable wavelength. 
However, the leakage current is usually significantly higher in a biased $n-i-n$ structure than that in a reversed-biased $p-i-n$ structure. Therefore, the electrical properties of the CQW sample should be measured to distinguish the effects of cross-well carrier transport and the physics of in-plane photogenerated Xs.

\subsubsection{Stationary photocurrent measurement}

\subsubsection{Dark current}

The low-temperature dark current $\left(\mathrm{I}_{\text {dark }}\right)$ of a mesa of $\sim(800 \mu \mathrm{m})^{2}$ area for $V_{b}>-$ $0.9 \mathrm{~V}$ is typically less than $100 \mathrm{pA}$ (Figure 7.1). Though the CQW sample has a designed overall $n$-i-n symmetric structure, $\mathrm{I}_{\mathrm{dark}}$ is asymmetric and increases significantly (similar to junction breakdown) for $V_{b}<-0.9 \mathrm{~V}$. All mesas of the CQW sample have a qualitatively similar $V_{b}$ dependence (not shown). For all mesas, $\left|I_{\text {dark }}\right|$ increases sharply for $V_{b}$ less than about $-1 \mathrm{~V}$; while for some mesas a sharp increase of $\left|\mathrm{I}_{\text {dark }}\right|$ is also observed for $V_{b}$ higher than about $+1 \mathrm{~V}$. The non-zero asymmetric $\mathrm{I}_{\text {dark }}-\mathrm{V}$ curves indicate that the grown CQW sample has an intrinsic asymmetric band structure in the growth direction (z-direction).

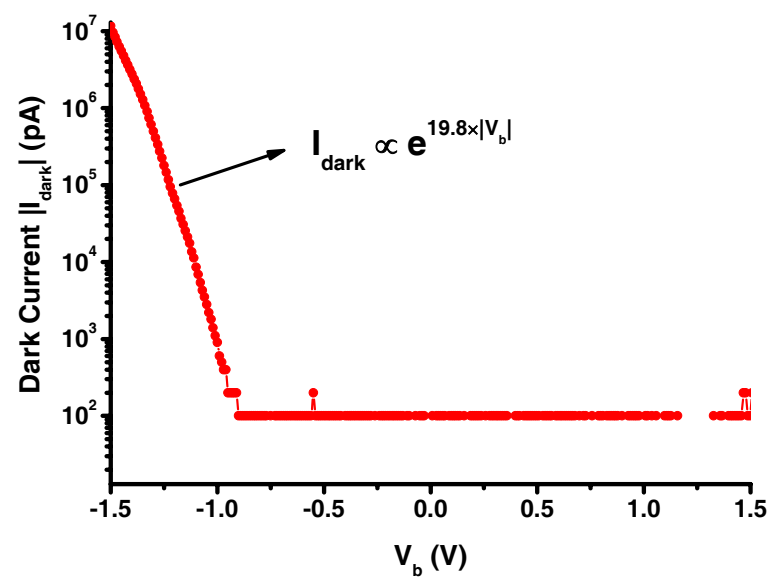

Figure 7.1 Current-voltage characteristics in the dark for a $\sim(800 \mu \mathrm{m})^{2}$ mesa. 


\subsubsection{Photocurrent-voltage characteristics}

The photocurrent-voltage (I-V) characteristics of the CQW sample were measured under a uniform or local tight-focused laser excitation as a function of the laser excitation power, $P_{\text {laser }}$. Figure 7.2 shows the low-temperature $(T=1.7 \mathrm{~K}) \mathrm{I}-\mathrm{V}$ curves measured under tightly-focused HeNe excitation at the center of the $\sim(800 \mu \mathrm{m})^{2}$ mesa that has been studied in this dissertation. These asymmetric I-V curves demonstrate that the devices behave like a p-n junction, although it was designed to be a overall symmetric $n^{+}-i-n^{+}$ structure. Moreover, as pointed out previously, the I-V curves exhibit negative differential resistance (NDR) regions around $V_{b}=-1 \mathrm{~V}$, indicative of carrier tunneling/transport across the CQW structure. The fragmented PL ring patterns appeared near the NDR region, which indicated that the appearance of the PL rings might be closely related to the cross-well carrier tunneling/transport.

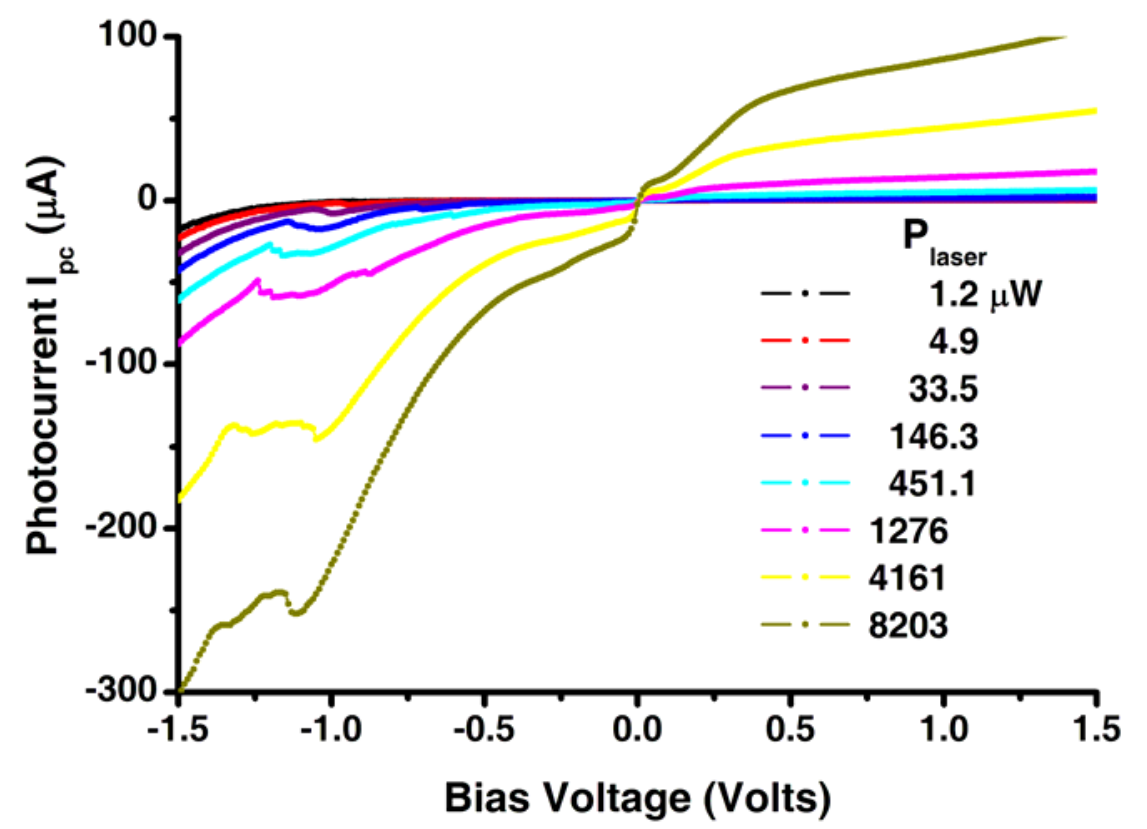

Figure 7.2 Photocurrent-voltage (I-V) characteristics under a local $\mathrm{HeNe}$ excitation at $T=1.7 \mathrm{~K}$ for $P_{\text {laser }}$ as indicated. (laser spot radius $\sigma_{\mathrm{r}} \approx 6 \mu \mathrm{m}$ ) 


\subsubsection{Photovoltaic effects}

The asymmetry of the grown CQW structure is also revealed from the weak photovoltaic effects observed. Figure 7.3 is a magnified view of the I-V curves around the origin, which shows non-zero short-circuit (i.e. $V_{b}=0$ ) photocurrent $\left(\mathrm{I}_{\mathrm{SC}}\right)$ flowing from the grounded bottom contact to the top contact (see Figure 3.1 for electrical connections). ISC increases linearly with $P_{\text {laser }}$ ( Figure 7.4: $\left.\mathrm{I}_{\mathrm{SC}} \propto\left(P_{\text {laser }}\right)^{0.96} \sim P_{\text {laser }}\right)$, which is a typical characteristic of a junction solar cell [183]. The saturation at high $P_{\text {laser }}$ is attributed to the reduction of photogenerated carriers caused by absorption saturation.

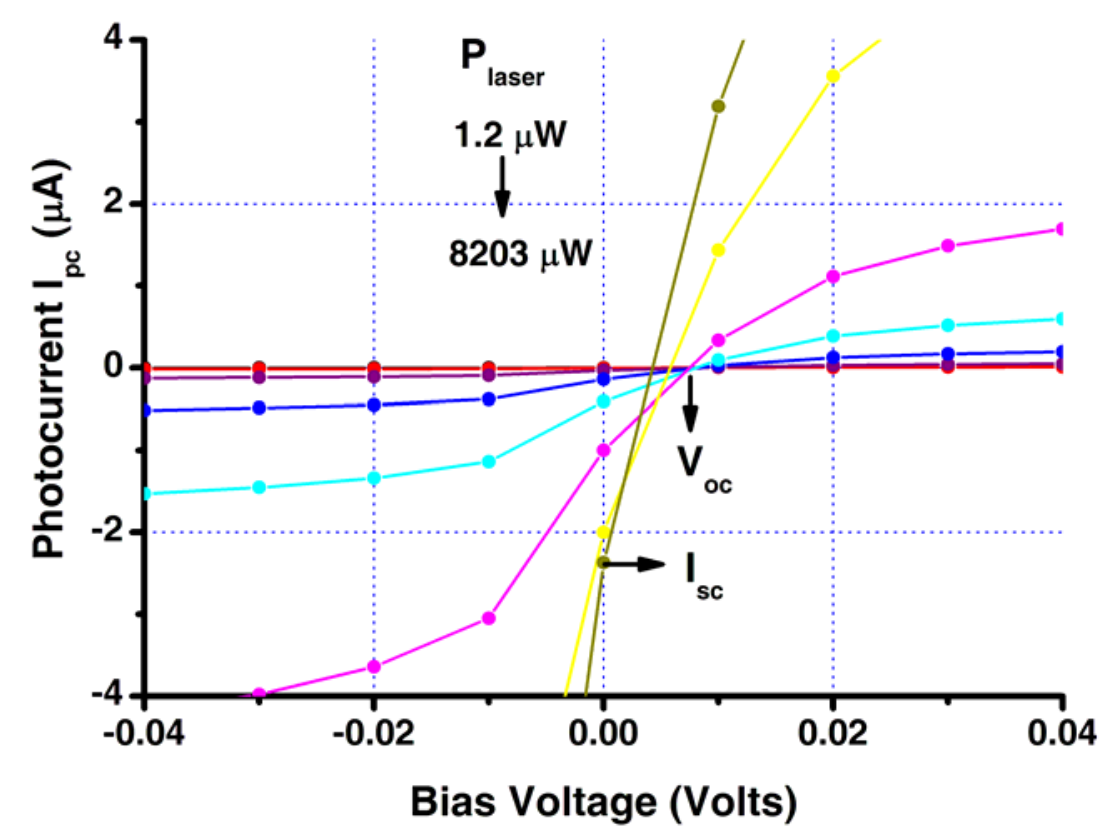

Figure 7.3 Zoom-in view near the origin of the photocurrent-voltage characteristics shown in Figure 7.2. The arrows point to the short-circuit current $\left(\mathrm{I}_{\mathrm{sc}}\right)$ and open-circuit voltage $\left(\mathrm{V}_{\mathrm{oc}}\right)$.

The weak photovoltaic effects indicate a built-in internal electric field when excited by a HeNe laser. This suggests an overall asymmetric band structure or doping in the z-direction, although the asymmetry is weak. One possible cause is an average lighter doping concentration in the top $\mathrm{n}^{+}$-GaAs layer (hence p-like with respect to the bottom 
layer), which results in a weaker bandgap narrowing. However, this asymmetric band structure can only explain the current-voltage characteristics for $V_{b} \approx 0$. The overall photocurrent-voltage characteristics actually suggest opposite asymmetric band structure with a p-like bottom layer. The photogenerated carriers clearly affect the band structure of the CQW sample.

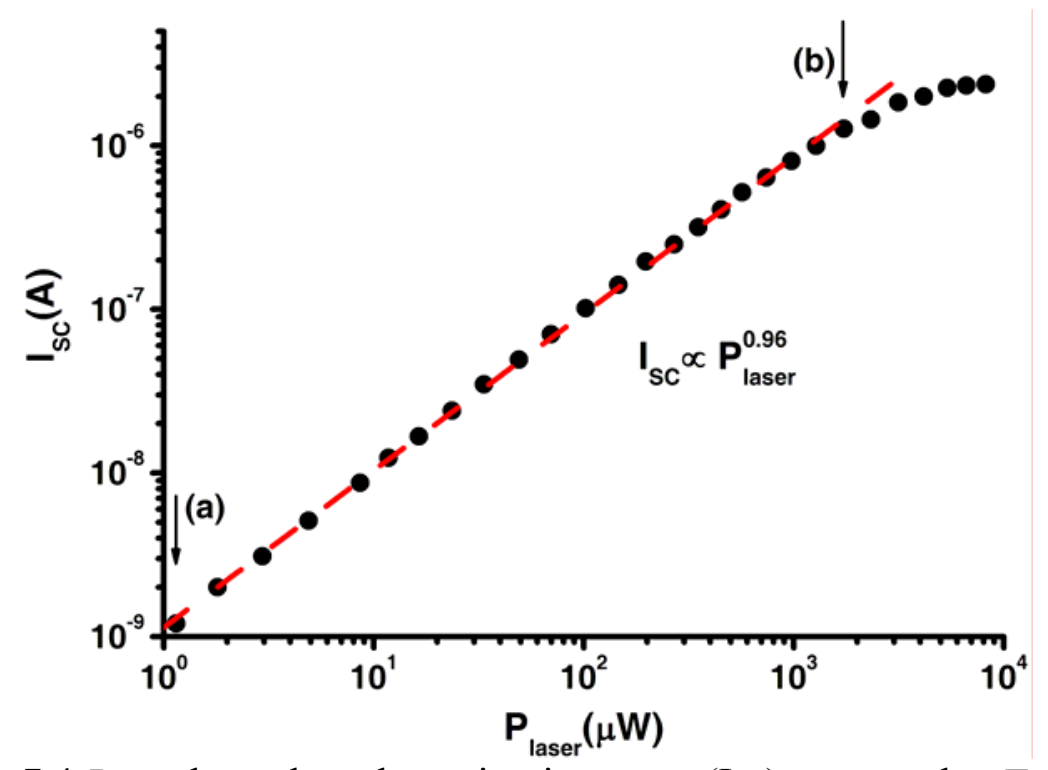

Figure 7.4 $P_{\text {laser }}$-dependent short-circuit current $\left(\mathrm{I}_{\mathrm{SC}}\right)$ measured at $T=1.7 \mathrm{~K}$ with a tight-focused $\mathrm{HeNe}$ laser spot (radius $\sigma_{\mathrm{r}} \sim 6 \mu \mathrm{m}$ ) exciting halfway between Trap-A and Trap-B. The dash line is the linear fit with data points between (a) and (b), which gives $\mathrm{I}_{\mathrm{SC}} \propto\left(P_{\text {laser }}\right)^{0.96} \sim P_{\text {laser }}$.

\subsubsection{Responsivity and photocurrent gain}

Following the convention used for photodetectors, we define responsivity $(\mathfrak{R})$ as

$$
\mathfrak{R}=\frac{\left|I_{p c}\right|}{P_{\text {laser }}}(A / W) .
$$

Here $I_{p c}$ is the net photocurrent, which was determined by subtracting the dark current (Figure 7.1) from the previously measured photocurrent (Figure 7.2). Figure 7.5 shows the responsivity ( $\Re$ ) versus $P_{\text {laser }}$ and $V_{b}$. 

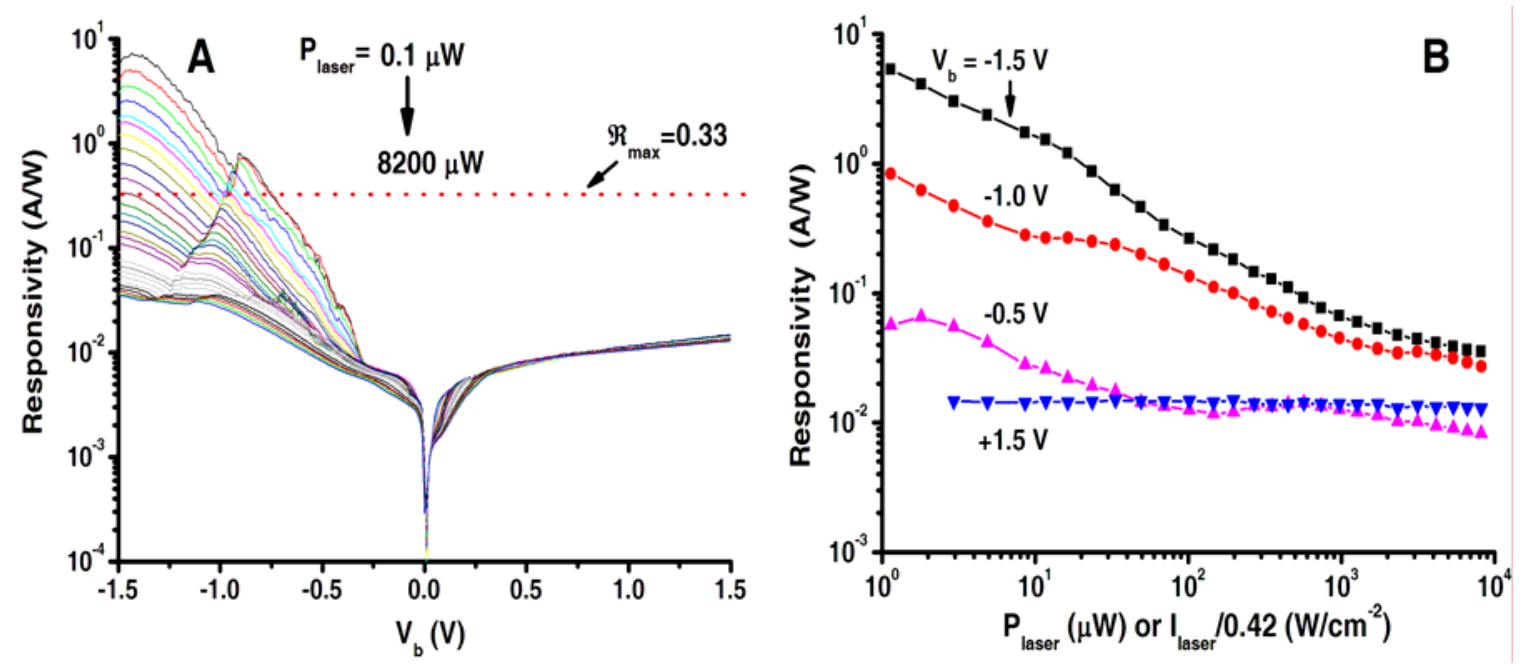

Figure 7.5 Responsivity $(\mathfrak{R})$ versus $P_{\text {laser }}$ and $V_{b}$ at $T=1.7 \mathrm{~K}$. A HeNe laser beam was tightly focused (spot radius $\sigma \approx 6 \mu \mathrm{m}$ ) on the center of the $\sim(800 \mu \mathrm{m})^{2}$ mesa. (A) $\mathfrak{R}\left(V_{b}\right)$ for $P_{\text {laser }}=0.1 \mu \mathrm{W}$ to $8200 \mu \mathrm{W}$. (B) $\mathfrak{R}\left(P_{\text {laser }}\right)$ for $V_{b}=-1.5,-1.0,-0.5$, and $+1.5 \mathrm{~V}$.

Because the CQW sample was designed to have an overall $n-i-n$ symmetric structure, the photocurrent responsivity is expected to exhibit similar to a reverse-biased p-i-n photodiode if leakage currents are negligible. This is the case for $V_{b}>-0.3 \mathrm{~V}$, where the dark current is below $10^{-10}$ A (Figure 7.5). For $-0.3 \mathrm{~V}<V_{b}<1.5 \mathrm{~V}, \mathfrak{R}$ is generally less than 0.01 and almost independent of $P_{\text {laser }}$ (Figure 7.5A). However, for $V_{b}<-0.5, \mathfrak{R}$ increases with decreasing $P_{\text {laser }}$ and can exceed a value of 10 . This is significantly higher than the typical responsivity of a $p-i-n$ photodiode, which has $\sim 0.2$ maximal responsivity for high-sensitivity ones.

Figure $7.5 \mathrm{~B}$ shows the responsivity $\mathfrak{R}$ as a function of $P_{\text {laser. }} \mathfrak{R}$ is independent of $P_{\text {laser }}$ for $V_{b}>-0.3 \mathrm{~V}$ as examplified by the $\mathfrak{R}\left(P_{\text {laser }}\right)$ curve at $V_{b}=+1.5 \mathrm{~V}$ (blue triangles). Responsivity $\mathfrak{R}$ increases non-linearly with descreasing $P_{\text {lase }}$ for negative $V_{b}<-0.5 \mathrm{~V}$ : the leakage current is also significant, even in the dark. The nonlinear $P_{\text {laser-dependent }}$ responsivity and high responsivity values for $V_{b}<-0.5$ and low $P_{\text {laser }}$ indicate that the photocarriers generated by the incident laser may not be the only source of the 
photocurrent. To better understand the cause of such high and nonlinear responsivity, we consider the photocurrent gain of the CQW sample.

The photocurrent gain $\left(\Gamma_{\mathrm{G}}\right)$ is related to the photocurrent $\left(\mathrm{I}_{\mathrm{pc}}\right)$ and generation rate of the photocarriers $(\mathrm{G})$ by

$$
I_{p c}=\Gamma_{G} q G
$$

G is given by

$$
G=\eta_{i} \frac{(1-R) P_{\text {laser }}\left(1-e^{-\alpha L}\right)}{h v} \quad(\# / \mathrm{sec}),
$$

where $\eta_{\mathrm{i}}(\approx 100 \%$ for high quality samples) is the internal quantum efficiency, $\mathrm{R}$ the surface reflectance, $\alpha$ the absorption coefficient, $L$ the thickness of the absorption region, and $h v$ the incident laser energy.

The gain $\left(\Gamma_{\mathrm{G}}\right)$ is thus associated with the responsivity $(\mathfrak{R})$ by

$$
\Re=\Gamma_{G} \times \eta_{i} \times \frac{q(1-R)\left(1-e^{-\alpha L}\right)}{h v} .
$$

The generation of photocarriers can be followed by one of the two processes: (a) successive re-injection of the faster carrier (see below), or (b) loss by recombination. In general, $\Gamma_{\mathrm{G}}<1$ if carriers recombine before being swept out, and $\Gamma_{\mathrm{G}}>1$ if re-injection can occur. For an ideal $n-i-n$ structure, all photogenerated carriers are swept out without replenishment by re-injection or recombination loss; therefore, the value of $\Gamma_{\mathrm{G}}$ is expected to be close to 1 if all photogenerated carriers contribute to the photocurrent. This is of course not the case for a CQW sample due to the recombination loss (photoluminescence), which reduces the value of $\Gamma_{\mathrm{G}}$. Nevertheless, $\Gamma_{\mathrm{G}}=1$ should be the upper bound if re-injection is negligible. 
For a HeNe laser $(\mathrm{h} v=1.959 \mathrm{eV})$ incident normally on the GaAs surface of the CQW sample, the transmittance $T=(1-R) \approx 0.65$. Assuming $\Gamma_{\mathrm{G}}=1, \eta_{\mathrm{i}}=100 \%$, and $\alpha \mathrm{L}=$ $\infty$ (i.e. all incident photons are absorbed by the sample and converted into the photocurrent), we obtain $\Re=0.33\left(\equiv \Re_{\max }\right.$ ), which is an absolute maximum responsivity value unless re-injection occurs. The responsivity clearly can exceed $\mathfrak{R}_{\max }$, which confirms the occurrence of carrier re-injection for $V_{b}$ about less than $-0.5 \mathrm{~V}$ under a HeNe laser excitation.

We now turn our attention to the possible causes of carrier re-injection.

The optical energy absorbed by the CQW device can be converted to electrical energy, which is usually manifest as a photocurrent flowing through the QW structure and the external circuit. The process usually involves several steps [183]: (i) Absorption of optical energy and generation of carriers, (ii) recombination of some of the photogenerated electron-hole pairs, (iii) transportation of the electrons and/or holes across the barriers in opposite directions through tunneling, thermionic emission, or direct sweep-out, and finally (iv) carrier collection or re-injection to generate a photocurrent flowing through the external circuitry.

Optical absorption in the depletion region of a semiconductor diode generates extra pairs of electrons and holes. These photogenerated carriers are swept out by the electric field, giving rise to a reverse current of one electron (or forward current of one hole) for every generated electron-hole pairs. It is noteworthy that the electron going in one direction and the hole going in the other are in the end the same current: only one particle passes any given point, so only one electronic charge travels around the circuit, not two. The resultant photocurrent will persist until both carriers are collected at the 
electrodes, or until they recombine (radiatively or nonradiatively ${ }^{20}$ ) in the structure before reaching the contact electrodes. The time for detection of the photocurrent is limited by the transit time of the faster carrier (usually the electrons) between the electrodes. Electrons travel close to their saturated drift velocity $\left(\sim 10^{7} \mathrm{~cm} / \mathrm{sec}\right.$ in GaAs or AlGaAs) [51] under a $\sim 25 \mathrm{kV} / \mathrm{cm}$ electric field, which is a typical field across the active region of the CQW sample studied. Thus, the time taken to sweep the carriers out under such a strong field is usually quite short ( 10 ps for one $\mu \mathrm{m})$. However, the continued persistence of the slower holes (or trapped carriers) will draw more electrons (or opposite-type carriers) to maintain charge neutrality. This gives rise to a photocurrent gain, $\Gamma_{\mathrm{G}}$, which can be approximated by

$$
\Gamma_{G} \approx \frac{\tau_{m}}{\tau_{t r}}
$$

where $\tau_{\mathrm{m}}$ is the carrier (the minority carrier) life time, and $\tau_{\mathrm{tr}}$ is the transit time of the fast electrons [183]. In order to calculate the photocurrent caused by absorption of light, we clearly have to find out how long the excess carriers exist between the electrodes before they are swept out or recombine, and consider the carrier injection through the contacts.

After understanding possible sources of photocurrent gain, we now examine the nonlinear $P_{\text {lase }}$-dependent photocurrent gain. Figure 7.5B shows that the responsivity ( $R$ ) decreases with increasing $P_{\text {lase }}$ for $V_{b}$ less than about $-0.5 \mathrm{~V}$. This could be qualitatively explained by a longer transit time $\left(\tau_{\text {tr }}\right)$ due to the decrease of the cross-well electric field caused by screening of photogenerated carriers or space charges. However, the carrier lifetime $\left(\tau_{\mathrm{m}}\right)$ can be affected by the electric fields and the space charges can cause

\footnotetext{
${ }^{20}$ The losses in active regions can occur due to free-carrier absorption, scattering at defects and other nonradiative transitions.
} 
nonlinear carrier transit across the CQW structure. These make quantitative analysis quite difficult at the current stage. Nevertheless, we found that the 'intrinsic' region of the CQW sample was not defect-free and might be photoconductive under $\mathrm{HeNe}$ laser excitation.

In the next section, we will describe the transient photocurrent response, which will provide stronger evidence for charge trapping, space-charge build-up, and persistent photoconductivity.

\subsubsection{Transient photocurrent}

We have described the asymmetric stationary I-V characteristics in Sec. 7.2.2. We measured the low-temperature $(T=1.7 \mathrm{~K})$ transient photocurrents $(\mathrm{TPC})$ due to a remote localized (radius $\sigma_{\mathrm{r}} \approx 8 \mu \mathrm{m}$ ) $10-20 \mathrm{~ns}$ pulsed excitation of energy $\mathrm{h} v=1.944 \mathrm{eV}$, which is above the bandgap of AlGaAs barriers. Figure 7.6 shows selected TPC measured at bias $V_{b}=-1 \mathrm{~V}$ and $+1 \mathrm{~V}$, which represent qualitatively the TPC for positive and negative $V_{b}$ 's. Pulsed laser excitation of $\sim 10$-ns duration was halfway between Trap-A and Trap-B, which is close to the center of the $\sim(800 \mu \mathrm{m})^{2}$ mesa. 


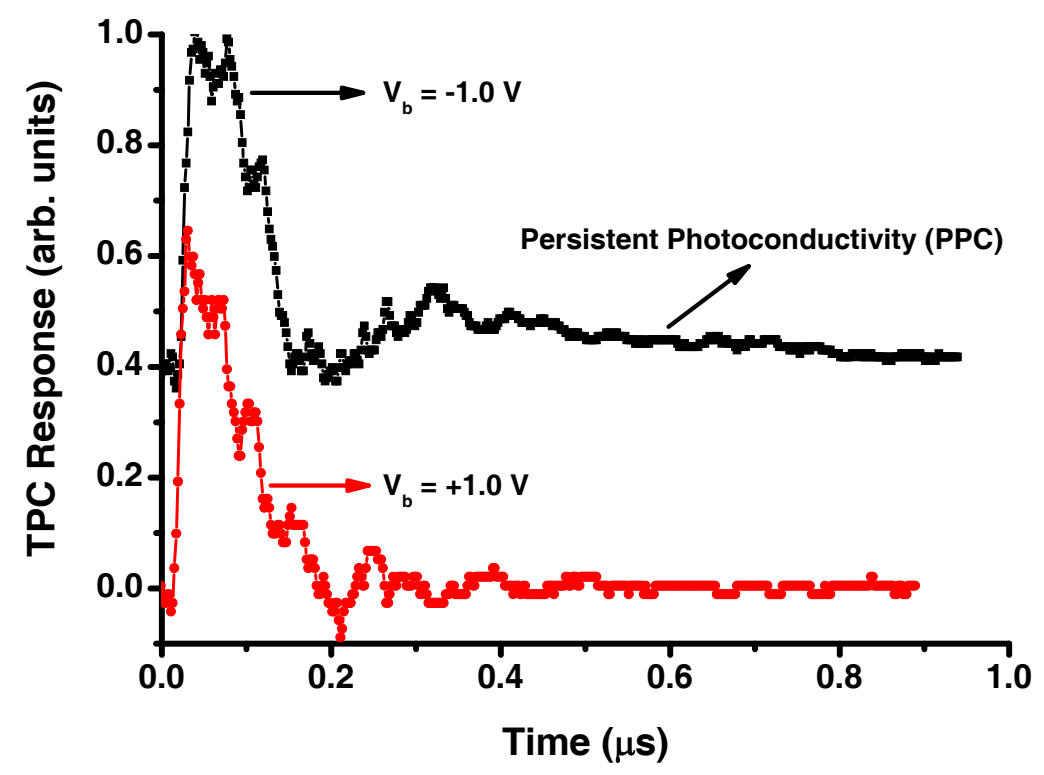

Figure 7.6 Transient photocurrent (TPC) at $V_{b}=-1$ and +1 V. (Laser diode excitation: spot radius $\sigma \approx 8 \mu \mathrm{m}, \mathrm{h} v=1.944 \mathrm{eV}$, repetition rate $=100 \mathrm{kHz}$, pulse duration $\sim 10 \mathrm{~ns}$, and average $P_{\text {laser }} \approx 10 \mu \mathrm{W}\left[I_{\text {laser }} \approx 2.5 \mathrm{~W} / \mathrm{cm}^{-2}\right]$ ).

For $V_{b}=+1 \mathrm{~V}$, the TPC response consists only of a $0.1-\mu$ s to $0.2-\mu$ s pulse; whereas for $V_{b}=-1 \mathrm{~V}$, the fast TPC response is followed by a long-decay component that can last up to about $10 \mu$ s (not shown). The long-decay photocurrent is likely associated with a persistent photoconductivity (PPC).

The conclusions of this observation are as follows:

(i) The initial transient PC pulse lasts much longer than the pulsed excitation.

(ii) For $\mathrm{V}_{\mathrm{b}}<0 \mathrm{~V}$, after the initial transient PC pulse there is a very long lived persistent photoconductivity.

These observations suggest that the initial PC pulse is due to the high energy photocarriers that transit the device; whereas the long lived PPC is related to some defects or trapping of charged carriers. 
If the photocarriers were just swept through the CQW/Barriers structure by the strong $(\sim 23 \mathrm{kV} / \mathrm{cm})$ applied field, the rise and fall of the TPC should be on the order of nanoseconds or less. The slow fall time $(\sim 100 \mathrm{~ns})$ implies a long carrier transit time, which can be caused by multiple scatterings of the hot carrier or trapping of photogenerated carriers. The long decay PPC component is unlikely to be caused by similar stronger carrier trapping and space-charge build-up mechanisms. One natural interpretation is that photocarriers are trapped by defects in the AlGaAs layers and then released gradually with a long time constant. However, this is not the only possibility.

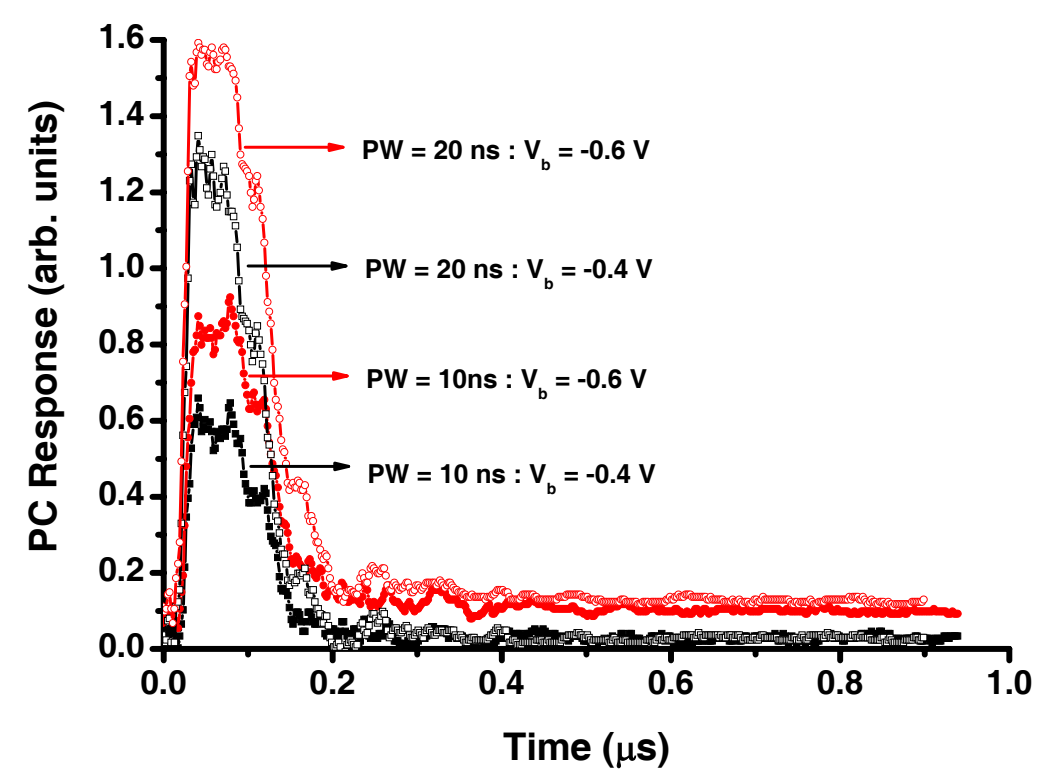

Figure 7.7 Transient photocurrent (TPC) measured with different pulse durations. (Laser diode excitation: spot radius $\sigma \approx 8 \mu \mathrm{m}, \mathrm{h} v=1.944 \mathrm{eV}$, repetition rate $=100 \mathrm{kHz}$, and average $P_{\text {laser }} \approx 10 \mu \mathrm{W}\left[I_{\text {laser }} \approx 2.5 \mathrm{~W} / \mathrm{cm}^{-}\right.$ $\left.\left.{ }^{2}\right]\right)$.

Figure 7.7 shows the PC response for excitation with 10 -ns and 20-ns laser pulses and for $V_{b}=-0.4 \mathrm{~V}$ (black curves) and $V_{b}=-0.6 \mathrm{~V}$ (red curves). The number of photon per pulses for the 20-ns case is about twice that for the 10-ns one. 
The initial transient PC pulses scale with the number of photons per pulse; whereas the PPC depends mainly on $V_{b}$. This implies that the PPC is not only due to the trapping and de-trapping of photocarriers. We thus measured the time-resolved photocurrent-voltage characteristics (I-V curves) of the device to examine other possibilities.

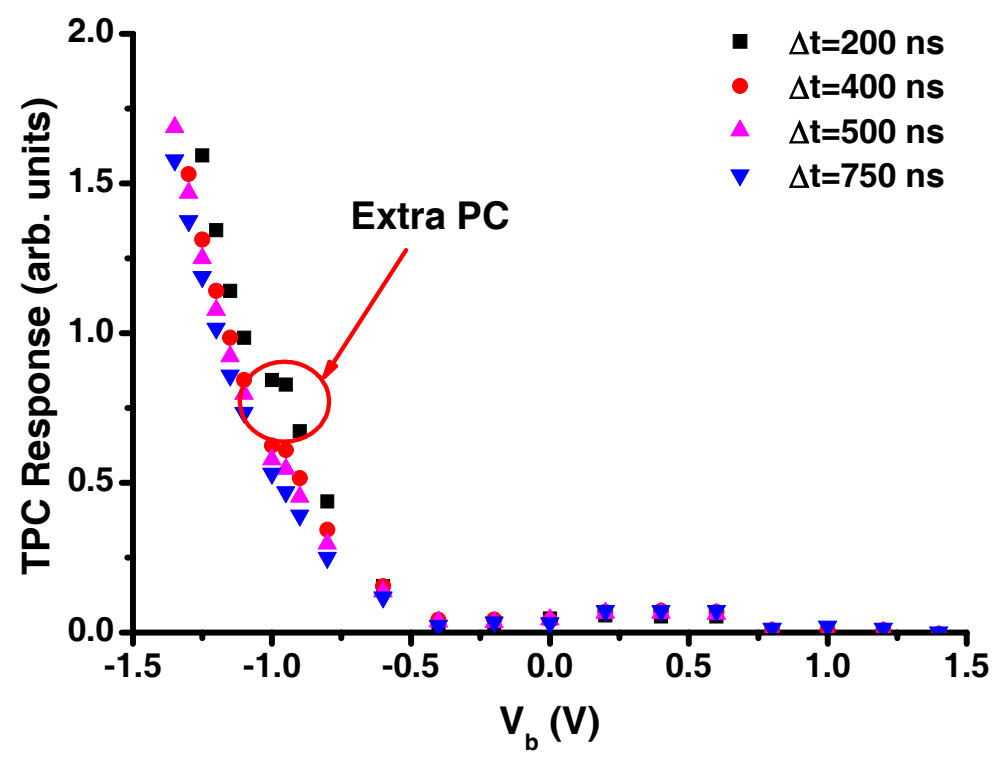

Figure 7.8 Time-resolved I-V characteristics at time delays $\Delta \mathrm{t}=200,400$, 500 , and $750 \mathrm{~ns}$, respectively. (Laser diode excitation: spot radius $\sigma \approx 8$ $\mu \mathrm{m}, \mathrm{h} v=1.944 \mathrm{eV}$, pulse duration $\approx 10 \mathrm{~ns}$, repetition rate $=100 \mathrm{kHz}$, and average $P_{\text {laser }} \approx 10 \mu \mathrm{W}\left[I_{\text {laser }} \approx 2.5 \mathrm{~W} / \mathrm{cm}^{-2}\right]$ )

Figure 7.8 shows the instantaneous amplitude of the TPC response in the PPC regime at $\Delta \mathrm{t}=200,400,500$ and $750 \mathrm{~ns}$ after the initial pulse as a function of $V_{b}$. PPC was observed for all accessible $V_{b}<0$ and for $V_{b}=0$ to about $+0.5 \mathrm{~V}$. The I-V curve at $\Delta \mathrm{t}$ $=200 \mathrm{~ns}$ (black squares) exhibits an increase at $V_{b} \approx-1 \mathrm{~V}$ (marked by the red circle in Figure 7.8).

We note two important points: (i) this increase of PC occurs exactly where the NDR is observed, (ii) it is delayed by $\sim 200 \mathrm{~ns}$ and lasts approximately $\sim 200 \mathrm{~ns}$ (Figure 7.6). We 
thus conclude that (i) the PPC cannot be explained simply by trapping and de-trapping, and (ii) the NDR is associated with additional carriers flowing through the device with a time of flight $\sim 200 \mathrm{~ns}$.

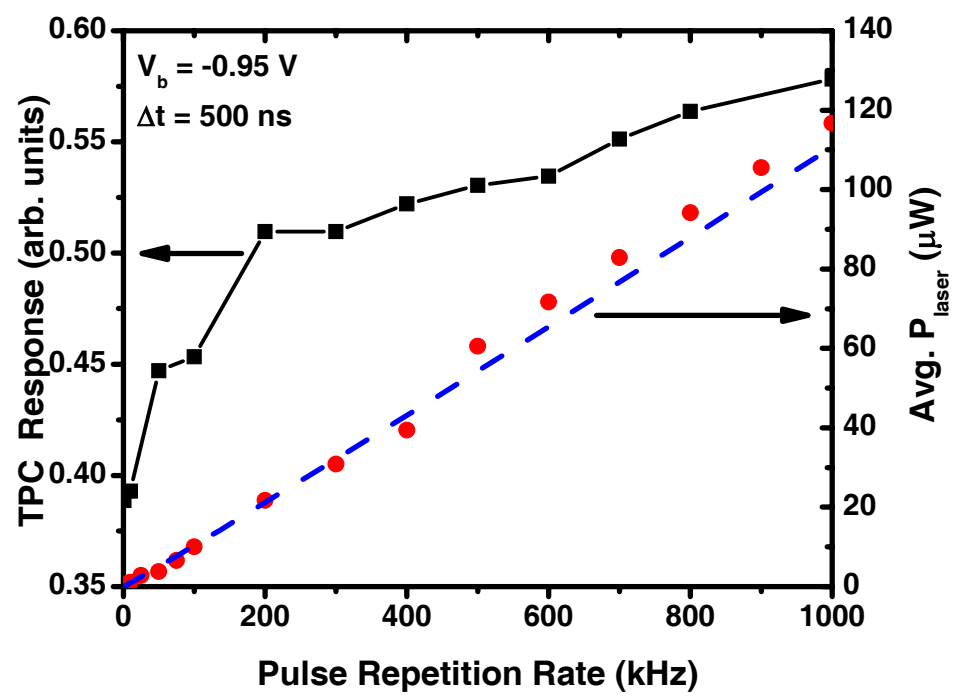

Figure 7.9 Transient photocurrent versus the laser repetition rate. (Laser diode excitation: spot radius $\sigma_{\mathrm{r}} \approx 8 \mu \mathrm{m}, \mathrm{h} v=1.944 \mathrm{eV}$ )

The PPC decay rate was determined by measuring the transient photocurrent amplitude at a fixed time delay $\Delta \mathrm{t}=500 \mathrm{~ns}$ following the pulse excitation (where the PPC dominates) as a function of the pulse repetition rate. The black squares in Figure 7.9 indicate the photocurrent response versus the laser repetition rate. It falls sharply for repetition rates smaller than about 10 to $100 \mathrm{kHz}$, which indicates a PPC decay time on the order of 10-100 $\mu$ s. In Figure 7.9, the solid red circles are the measured average laser power $P_{\text {laser }}$, and the blue dashed line is a linear fit. This excellent linear $P_{\text {laser }}$ dependence confirms that the sharp fall of photocurrent response is due to the increase of the time spacing between consecutive pulses, not a variation in $P_{\text {laser }}$. 
We have observed an unusual long decay time $\left(\tau_{\mathrm{m}}\right)$ of photogenerated carrier and persistent photoconductivity in the CQW sample studied. These results agree with the gain observed from stationary photocurrent measurements. Because current gain $\Gamma_{\mathrm{G}} \approx$ $\tau_{\mathrm{m}} / \tau_{\mathrm{tr}}$, a long carrier lifetime $\left(\tau_{\mathrm{m}}\right)$ will cause a current gain due to carrier re-injection. The measured photocurrents are thus composed of not only photogenerated carriers but also injected carriers.

These can also be understood from another perspective. DX (deep-level complex) centers in doped AlGaAs [184-186] have been known to give rise to persistent photoconductivity [187-189]. The AlGaAs barrier layers of the CQW sample were designed as undoped layers, thus it remains unclear if the photoconductivity is related to DX centers. A more thorough set of $T$-dependent current transient measurements $[190,191]$ is necessary to verify and clarify deep levels in AlGaAs. However, the PPC can be understood from a simple intuitive phenomenological model based on dielectric relaxation. The mesa studied has an area $A \approx(1050 \mu \mathrm{m} \times 920 \mu \mathrm{m})$, and the thickness between the heavily doped $\mathrm{n}^{+}$-GaAs metallic contacts $\mathrm{W}$ is about $0.66 \mu \mathrm{m}$. This gives a plate capacitance $C=\frac{\varepsilon A}{W} \approx 160 p F$ (the low-temperature dielectric constant $\varepsilon=12.4 \varepsilon_{0}$ ). The differential resistance $\left(R_{\text {diff }}\right)$ can be determined from the measured I-V curves, which is about $10^{4}$ to $10^{5} \Omega$ for $V_{b} \approx-1$ V. $\tau_{D}=R_{\text {diff }} \times C$ is the dielectric relaxation time along the z-direction. $\tau_{\mathrm{D}}$ is thus about $1.6 \mu$ s to $16 \mu \mathrm{s}$, which is close to the decay time of the observed PPC. Such dielectric relaxation is one signature of presence of space charges $[192,193]$. 


\subsection{Confined exciton systems}

\subsubsection{Electrical properties}

We have described the photocurrent-voltage (I-V) characteristics of the CQW sample under a uniform or local laser excitation. To study the spatial opto-electrical properties of the sample, the photocurrent $\left(\mathrm{I}_{\mathrm{pc}}\right)$ was measured by scanning a tightly focused HeNe laser excitation spot $(\sim 15 \mu \mathrm{m}$ in diameter $)$ across the mesa. These experiments reveal the electrical activity of 'traps', where dense indirect-Xs were formed in confine small areas (see Chapter 5).

Figure 7.10 shows the $\mathrm{I}_{\mathrm{pc}}$ measured at $V_{b}=-0.95 \mathrm{~V}$ by scanning the HeNe laser spot across the Trap-A (see Figure 5.1 for the image of the mesa) over a $\sim 600 \mu \mathrm{m}$ distance. $I_{p c}$ depends strongly on the excitation location of the laser spot and increases sharply when the laser spot was moved close to Trap-A. However, $\mathrm{I}_{\mathrm{PC}}$ decreases when the excitation spot is moved closer to Trap-B (not shown). We examined various mesas with similar traps, and found that $\mathrm{I}_{\mathrm{pc}}$ exhibits a spatial dependence and usually increases when the excitation is moved closer to traps, indicating that the 'traps' are indeed electrically active. Current filaments and injection or trapping of carriers (electrons or holes) could occur at these localities.

$\mathrm{I}-\mathrm{V}$ characteristics were also measured with laser excitation at different locations (Figure 7.11). When the laser excitation spot was moved closer to a 'trap' site, a higher $P_{\text {laser }}$ was necessary to observe the NDR described previously (Sec. 7.2.2). It is also noteworthy that PL emissions from other locations of the mesa disappeared when the excitation spot was on such an active 'trap' (Trap-A or Trap-B on this mesa). 


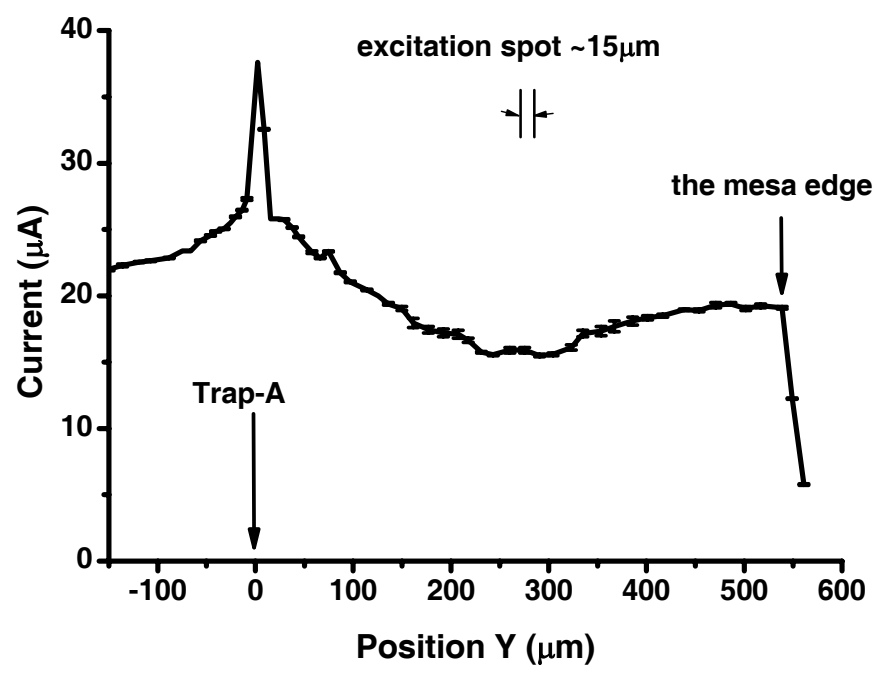

Figure 7.10 Photocurrent versus laser excitation locations. ( $T=1.7 \mathrm{~K}, V_{b}=$ $-0.95 \mathrm{~V}, \mathrm{HeNe}$ excitation spot diameter $\sim 15 \mu \mathrm{m}$ and $P_{\text {laser }}=0.35 \mathrm{~mW}$ ).

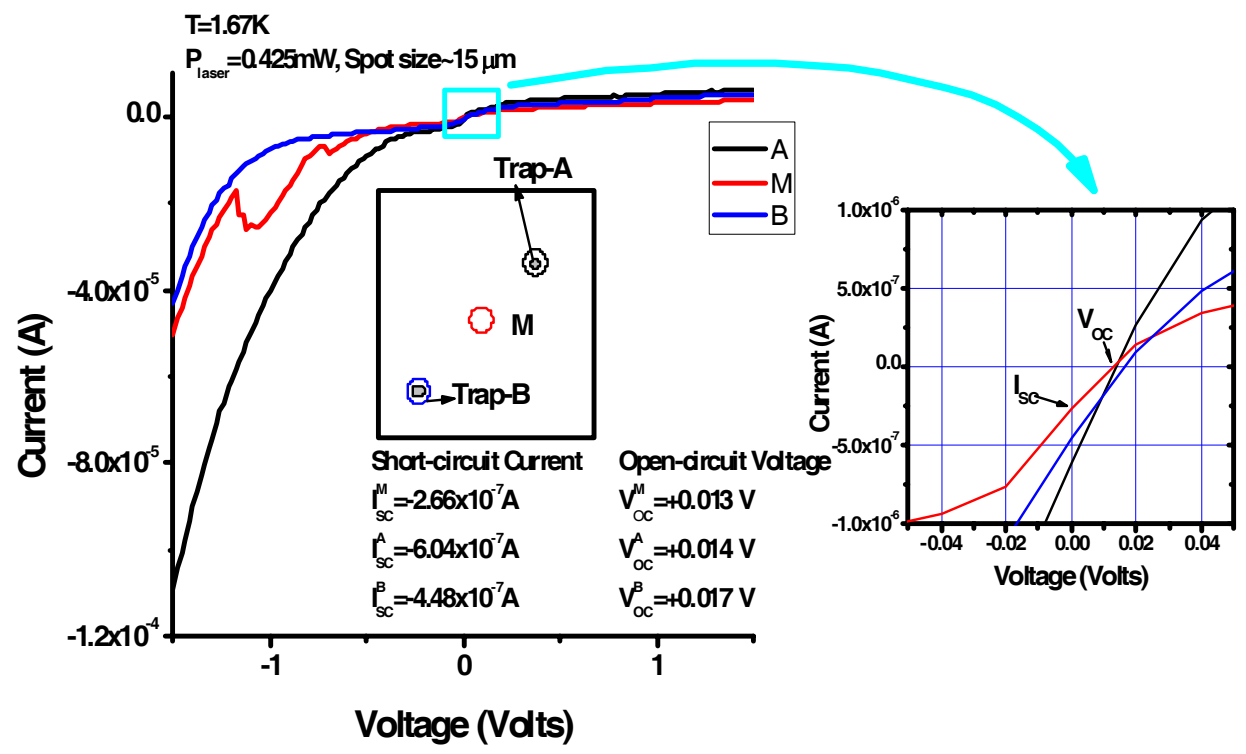

Figure 7.11 Photocurrent-voltage characteristics under a local $\mathrm{HeNe}$ excitation near the 'traps'. (spot diameter $\sim 15 \mu \mathrm{m}$, and $P_{\text {laser }} \approx 0.4 \mathrm{~mW}$ ). The inset is the zoom-in view near the origin. 


\subsubsection{Optical properties}

\subsubsection{Zero-field measurement}

We have described the photoluminescence properties of indirect-X systems in confined small areas in Chapter 5. To better understand the X collection mechanisms in these 'traps', we first characterized the sample under zero applied electric fields (Figure 7.12), where direct-X PL dominates and the localized indirect-X cloud disappears. QW structures grown by the molecular beam epitaxy have typical thickness fluctuations on the order of one monolayer, corresponding to about a $0.5-\mathrm{meV}$ energy variation for our 8$\mathrm{nm}$ QWs. ${ }^{21}$ The direct-X PL peak has a constant energy across the trap within our $\sim 0.1$ meV resolution; therefore, QW thickness fluctuation is unlikely to be the main cause of collection. A weak photocurrent is also observed, and at the trap center, a broad (linewidth $\Gamma \approx 10 \mathrm{meV}$ ) low intensity emission at $\sim 1.565 \mathrm{eV}$ is seen. This signals the presence of local inhomogeneous fields and/or current flows. Using the PL from the heavily doped $n+-G a A s$ layer, we determine that the Fermi level lowered by $\sim 1 \mathrm{meV}$ at the trap center. This indicated a lower local electron concentration which may be related to a variation in doping/vacancies or a photogenerated carrier drain.

\footnotetext{
${ }^{21}$ The energy states in CQW were calculated with 1D Poisson - a freeware program written by Gregory Snider for calculating energy band diagrams for semiconductor structures. The program can be downloaded from http://www.nd.edu/ gsnider/.
} 


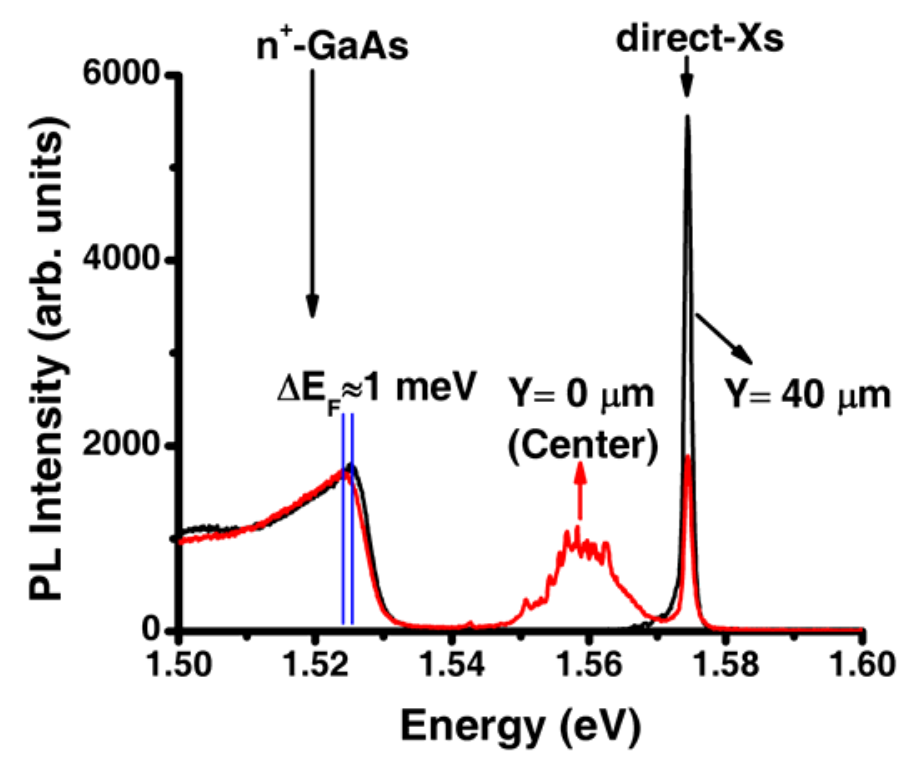

Figure 7.12 Spatially-resolved PL spectra taken at $V_{b}=0$ for $\mathrm{Y}=0$ and 40 $\mu \mathrm{m}$, where $\mathrm{Y}$ is the distance from the center of Trap-A.

\subsubsection{Electric field dependence}

In Sec. 7.2.2, we showed that the photocurrent depended upon the distance between the excitation spot and traps, which implies formation of current filaments through trap sites. The traps' electrical activity was confirmed by the electric field dependent PL shown in Figure 5.17A and B for Trap-A. The linear energy shift of well defined PL peaks seen for $-1 \mathrm{~V}<V_{b}<-0.5 \mathrm{~V}$ confirms the formation of well-defined indirect-Xs. This range of $V_{b}$ varies slightly under different $P_{\text {laser }}$ or for different traps. Only under these bias voltages does the $\mathrm{X}$ cloud contract and expand as described previously.

\subsubsection{Excitation energy dependence}

In the experiments presented thus far e-h pairs are photogenerated uniformly over a large area by a HeNe laser at hy $=1.96 \mathrm{eV}, \sim 20 \mathrm{meV}$ above the $\mathrm{Al}_{0.33} \mathrm{Ga}_{0.67} \mathrm{As}$ barrier bandgap $\left(\mathrm{E}_{\mathrm{gb}}\right)$ [51]. The effects reported in this chapter are readily observed for excitation energy $\mathrm{h} v$. between $\mathrm{E}_{\mathrm{gb}}$ and $\mathrm{E}_{\mathrm{ub}} \approx 2.34 \mathrm{eV}$. Importantly, they disappear abruptly as $\mathrm{h} v$ is 
tuned below $\mathrm{E}_{\mathrm{gb}}$ or above $\mathrm{E}_{\mathrm{ub}}$, even though the excitation is adjusted to create approximately the same density of e-h pairs in the CQW. This indicates the vital role of (i) the photocarriers generated in the AlGaAs barriers layers, and (ii) the difference in carrier capture by and escape from GaAs QWs.

\subsection{Photocurrents versus photoluminescence}

\subsubsection{General spatially indirect exciton systems}

In Sec. 7.2, we have shown that the I-V chacteristics of the CQW sample deviated from those of an ideal $n^{+}-i-n^{+}$structure because of (i) significant leakage photocurrents for $V_{b}<-0.5 \mathrm{~V}$ (more than 1000 time than that at $V_{b}=0$ ), (ii) photovoltaic effects, and (iii) photocurrent gains caused possibly by long-lifetime minority carriers or charges trapped in the 'intrinsic' region.

Here, imposing the law of energy conservation, we will show that the number of emitted indirect-X PL photons in this CQW sample can exceed the number of photons absorbed in two GaAs QWs. For these conditions, distant photoluminescence (DPL) emissions were clearly observed. This implies that a significant fraction of the DPL emission is due to carriers injected into the CQW.

The optical energy absorbed by the CQW sample dissipate through the following channels: (i) Photoluminescence - including the radiative recombination of Xs in QWs, carriers in $\mathrm{n}^{+}$-GaAs, recombination centers if any, and possible recombination of carriers in AlGaAs barrier layers under an excitation energy above its bandgap. (ii) Photocurrent

- including carrier diffusion and drift (sweep-out) in the presence of a cross-well electric field. (iii) Nonradiative recombination - including, for example, trapping of space charges, recombination centers, or Auger processes. For the latter energy could dissipate 
via phonons (i.e. heating), and build-up of space charges can in turn affect the measured current as discussed in Sec. 7.2.2.4.

For channel (i)-photoluminescence, we monitored X PL, $\mathrm{n}^{+}$-GaAs PL, and checked possible PL from AlGaAs or other defect radiative recombination centers. For channel (ii)-photocurrent, which is related to cross-well carrier transport, we considered the following cross-well carrier transport mechanisms [194-198]: (1) Direct tunneling from the lowest quantum state, (2) thermally assisted tunneling by thermal excitation from the first to a higher level followed by tunneling out of the well, (3) thermionic emission directly over the potential barrier, (4) trapping, and (5) drift (or sweep-out). For channel (iii)-nonradiative recombination, we neglected the Auger processes or heating effects.

First, we studied cases for $V_{b}=0$ and $-0.95 \mathrm{~V}$ under a uniform HeNe excitation at $T=1.7 \mathrm{~K}$. Figure 7.13 shows a comparison of the number of carriers deduced from the measured photocurrents with the estimated $\mathrm{X}$ photons emitted as a function of $P_{\text {laser }}$ under a uniform $\mathrm{HeNe}$ excitation (spot diameter $>500 \mu \mathrm{m}$ ). 


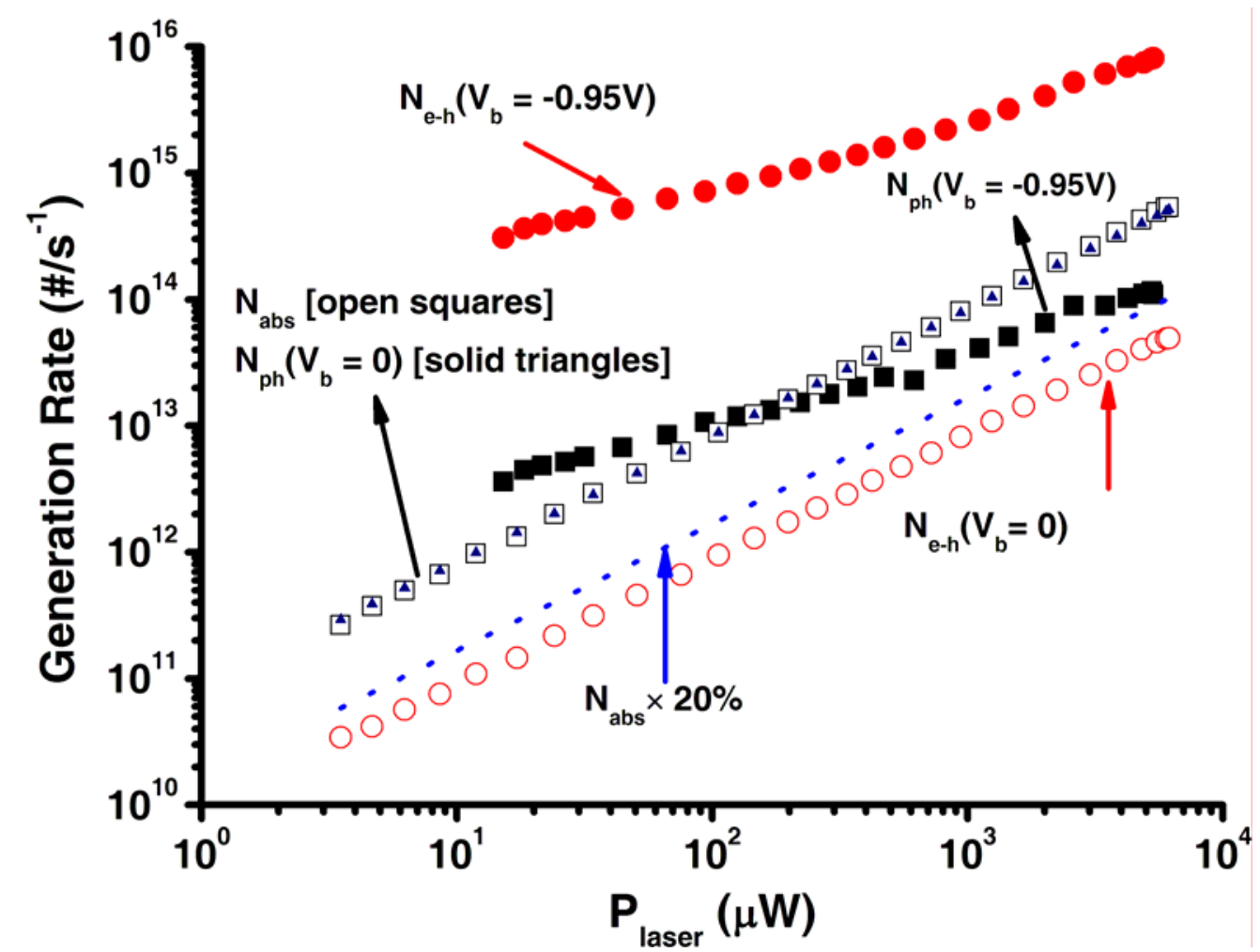

Figure 7.13 Estimated $P_{\text {laser }}$-dependent number of carriers and emitted $\mathrm{X}$ photons under a uniform HeNe excitation at $T=1.7 \mathrm{~K}$ and $V_{b}$ as indicated.

The symbols and curves in Figure 7.13 represent the followings:

a) The solid and open red circles correspond to e/h number flow through the device per second $\left(\mathrm{N}_{\mathrm{e}-\mathrm{h}}\right)$ deduced from the measured photocurrent for $V_{b}=-0.95 \mathrm{~V}$ and $V_{b}$ $=0$, respectively.

b) The open black squares represent the generation of electron-hole (e-h) pairs per second $\left(\mathrm{N}_{\mathrm{abs}}\right)$ in the two GaAs QWs calculated from the known GaAs absorption coefficient at low $T$ for total $16-\mathrm{nm}$ thickness of the two QWs, assuming $100 \%$ internal quantum efficiency.

c) The solid triangles inside the open black square label the number of estimated direct-X PL photons per second determined from the integrated signals of the PL CCD images ( $\left.\mathrm{I}_{\mathrm{PL}}\right)$ measured at $V_{b}=0$, using the system photon collection efficiency $\left(\mathrm{C}_{\text {eff }}\right)$ described in Sec. 4.5.2. 
d) The black solid squares represent the number of emitted indirect-X photons per second $\left(\mathrm{N}_{\mathrm{ph}}\right)$ at $V_{b}=-0.95 \mathrm{~V}$, which was again determined from $\mathrm{I}_{\mathrm{PL}}$ and the $\mathrm{C}_{\text {eff. Note that }}$ the absolute value of $\mathrm{C}_{\text {eff }}$ has no impact on the relative values of $\mathrm{N}_{\mathrm{ph}}\left(V_{b}=-0.95\right)$ and $\mathrm{N}_{\mathrm{ph}}\left(V_{b}=0\right) . \mathrm{N}_{\mathrm{ph}}\left(V_{b}=-0.95\right) / \mathrm{N}_{\mathrm{ph}}\left(V_{b}=0\right)$ is determined by the relative CCD image signal strength, which is independent of $\mathrm{C}_{\mathrm{eff}}$.

e) The blue dashed line is obtained by multiplying curve (b) by 0.2 and can be used as a guide to account for eventual loss in the collection system. This gives a lower estimate for the number of absorbed photons per second $\left(\mathrm{N}_{\mathrm{abs}}\right)$ in the two GaAs QWs. $\mathrm{N}_{\mathrm{abs}}$ should lie between the curves (b) and (e).

Next, we divided the observations into two parts: (i) $V_{b}=0 \mathrm{~V}$ and (ii) $V_{b}=-0.95 \mathrm{~V}$.

(i) $V_{b}=0 \mathrm{~V}$ (short-circuit):

Photocurrent - At $V_{b}=0$, the short-circuit photocurrents $\left(\mathrm{I}_{\mathrm{sc}}\right)$ are nonzero but increase linearly with increasing $P_{\text {laser }}$ (Figure 7.4). $\mathrm{N}_{\mathrm{e}-\mathrm{h}}=\mathrm{I}_{\mathrm{sc}} / \mathrm{q}$ is approximately $10 \%$ or less than $\mathrm{N}_{\mathrm{abs}}$; therefore, sweep-out of photogenerated carriers at $V_{b}=0$ is negligible. This justifies the low carrier loss (i.e. $\mathrm{N}_{\mathrm{abs}}=\mathrm{N}_{\mathrm{ph}}$ ) assumption used to determine system photon throughput from direct-X PL as described in Sec. 4.5.2.

Photons $-\mathrm{N}_{\mathrm{ph}}\left(V_{b}=0\right)$ has an excellent linear dependence on $P_{\text {laser }}$ ranging over three orders of magnitude. This shows that, at $V_{b}=0 \mathrm{~V}$, non-radiative recombinations are negligible or independent of $P_{\text {laser. }}$

(ii) $V_{b}=-0.95 \mathrm{~V}$ :

Photocurrent - At $V_{b}=-0.95 \mathrm{~V}, \mathrm{~N}_{\mathrm{e}-\mathrm{h}}$ is three orders of magnitude higher than that at $V_{b}=0$ and grows nonlinearly with $P_{\text {laser. }}$ This photocurrent gain and its causes have been discussed in Sec. 7.2.2.4 and Sec. 7.2.3. The nonlinear dependence of $\mathrm{N}_{\mathrm{e}-\mathrm{h}}$ on $P_{\text {laser }}$ 
can be attributed to a nonlinear growth of current gain with decreasing $P_{\text {laser }}$, which was also discussed in Sec. 7.2.3.

Photons - For $P_{\text {laser }}<100 \mu \mathrm{W}, \mathrm{N}_{\mathrm{ph}}\left(V_{b}=-0.95 \mathrm{~V}\right)$ clearly exceeds $\mathrm{N}_{\mathrm{abs}}$, which implies capture of extra carriers by the CQW. Hot photogenerated carriers can be swept out under high electric fields and results in a loss as indicated by the $\mathrm{N}_{\mathrm{abs}} \times 0.2$ curve showing a lower bound of $\mathrm{N}_{\text {abs. }}$ Therefore, the enhancement of $\mathrm{N}_{\mathrm{ph}}$ can be larger. Furthermore, $\mathrm{N}_{\mathrm{ph}}\left(V_{b}=-0.95 \mathrm{~V}\right)$ is not linearly dependent on $P_{\text {lase }}$, but follows the $\mathrm{N}_{\mathrm{e}-\mathrm{h}}$ dependence. This suggests that a significant fraction of the indirect-Xs detected is due to the capture of the cross-well transport carriers. Recall that at $V_{b}=-0.95 \mathrm{~V}$, indirect-Xs with small areas ('traps') were observed (see Chapter 5). $\mathrm{N}_{\mathrm{ph}}$ can exceed $\mathrm{N}_{\mathrm{abs}}$ by a greater amount for $V_{b}<-1.0 \mathrm{~V}$, where PL ring patterns and other local PL centers can be observed (see Chapter 6).

Comparing the numbers of the photoexcited carriers and the photons from $\mathrm{X}$ annihilations (direct or indirect ones) in QWs, and the swept-out electrons (holes), we conclude that carriers injected into or captured by the two GaAs quantum wells contribute significantly to the enhancement of indirect-X PL intensities for $V_{b}<-0.95 \mathrm{~V}$, where distant PL ('traps' and PL rings) can be readily observed under a HeNe laser excitation.

This conclusion raises further questions: (a) Are the carriers captured by the QWs photoexcited in the $\mathrm{n}^{+}$-GaAs layers and/or the AlGaAs barriers next to QWs? Or are they re-injection carriers induced by photoexcitation? (b) What are the effects of the carriers that are photoexcited in the AlGaAs barrier layers? 
We addressed these questions by comparing the $V_{b}$-dependent total integrated PL ( $\mathrm{I}_{\mathrm{PL}}$, including PL from $\mathrm{X}$ and $\left.\mathrm{n}^{+}-\mathrm{GaAs}\right)$ under a $\mathrm{HeNe}(\mathrm{h} v=1.959 \mathrm{eV})$ and a laser diode $(\mathrm{LD} 690, \mathrm{~h} v=1.802 \mathrm{eV})$ uniform excitation. The total integrated PL were determined from PL images taken with a long-pass filter allowing high transmission $(>90 \%)$ for photons of wavelengths $\lambda>715 \mathrm{~nm}(=1.734 \mathrm{eV})$ (Schott Glass RG715). The powers of the HeNe and LD690 lasers were adjusted to give approximately equal incident photon flux on the CQW sample.

The low-temperature $\left(\mathrm{T} \approx 2 \mathrm{~K}\right.$ ) bandgap of the $\mathrm{Al}_{0.33} \mathrm{Ga}_{0.67} \mathrm{As}$ barrier is $\sim 1.940 \mathrm{eV}$ ( $\left(\mathrm{E}_{\mathrm{gb}}\right.$ ), corresponding to a wavelength $\sim 639 \mathrm{~nm}$. Under HeNe laser excitation, we found no PL emissions from the CQW sample near that wavelength even under a zero bias. Radiative recombinations of the carriers photoexcited in the AlGaAs barriers (total thickness $\sim 400 \mathrm{~nm}$ ) thus are negligible. Therefore, by measuring the PL from the $\mathrm{n}^{+}-$ GaAs layers and Xs as well the photocurrent, we caould monitor the major dissipating channels of the energy deposited by laser excitation. Here we neglect energy dissipation in the form of phonons (i.e. heating) via nonradiative recombination.

We now examine the $V_{b}$-dependent $I_{P L}$ in accordance with the law of energy conservation. Figure 7.14 shows that $\mathrm{I}_{\mathrm{PL}}$ under HeNe excitation $\left(\mathrm{I}_{\mathrm{PL}}: \mathrm{HeNe}\right.$, red dots) is about twice $\mathrm{I}_{\mathrm{PL}}$ under LD690 excitation (IPL:LD690, black squares) for similar incident photon fluxes. This indicates that carriers photoexcited in the AlGaAs barriers contribute significantly to the observed PL. Under LD690 excitation, IPL is almost independent of $V_{b}$, but decreases and then increases slightly with increasing $\left|V_{b}\right|$. The weak quenching of $\mathrm{I}_{\mathrm{PL}}$ is attributed to an increase fraction of nonradiative recombination; whereas the weak enhancement of $\mathrm{I}_{\mathrm{PL}}\left(\sim 1.4 \%\right.$ at $V_{b}=-1.5 \mathrm{~V}$ as compared to that at $\left.V_{b}=0\right)$ can only be 
accounted for by carrier re-injection since the photocurrent increased with $\left|V_{b}\right|$ as well (see below). However, the re-injection effect is weak (particulary in comparison with the systemic errors) for an excitation below the AlGaAs bandgap $\mathrm{E}_{\mathrm{gb}}$, such as with LD690.

The re-injection effect is more pronounced under HeNe excitation. When $V_{b}$ was varied from 0 to $-1.5 \mathrm{~V}, \mathrm{I}_{\mathrm{PL}}$ increased almost monotonically up to $\sim 5.8 \%$ while the photocurrent increased by two orders of magnitude. These results indicate that carrier reinjection gives a vital contribution to the total PL emission. The enhancement of $\mathrm{I}_{\mathrm{PL}}$ is more significant at a large negative bias $V_{b}$, where photocurrents are significant and DPL readily observed.

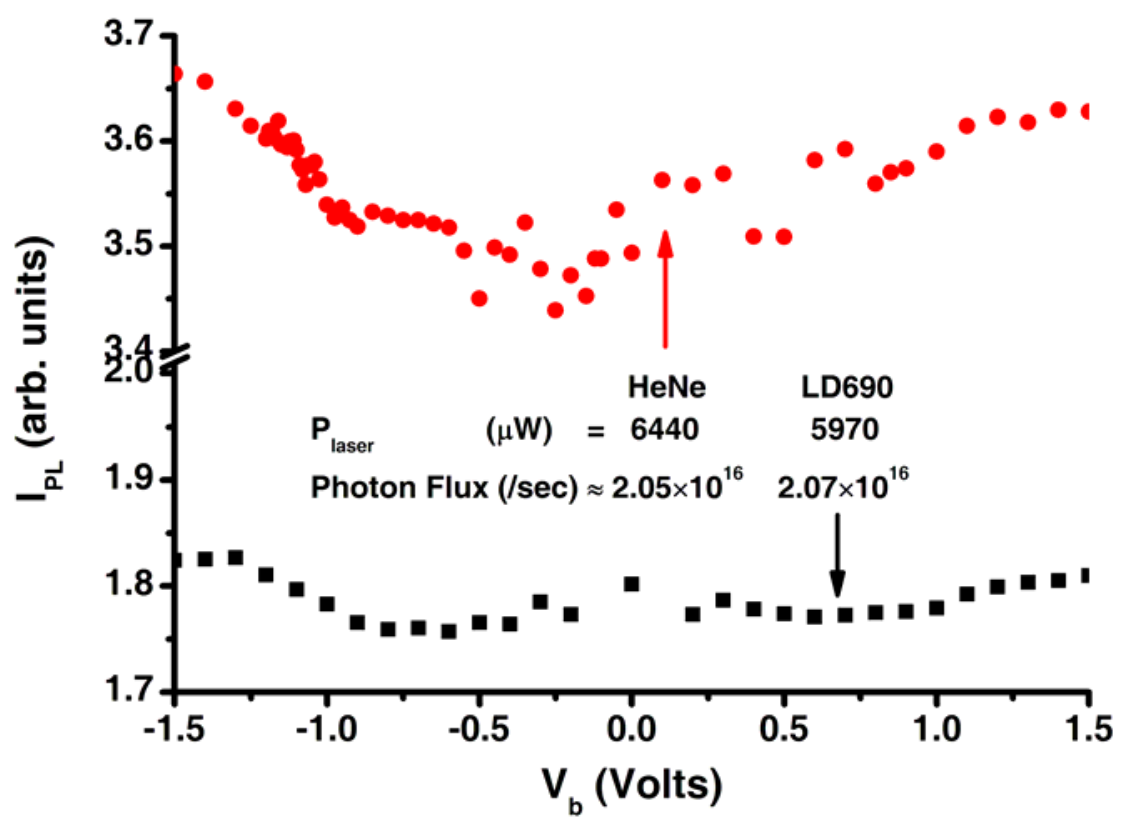

Figure 7.14 Comparison of $V_{b}$-dependent total integrated PL (including PL from $\mathrm{X}$ and $\mathrm{n}^{+}-\mathrm{GaAs}$ ) determined from PL imaging under a $\mathrm{HeNe}(\mathrm{h} v=$ $1.959 \mathrm{eV})$ and laser diode $(\mathrm{LD} 690, \mathrm{~h} v=1.802 \mathrm{eV})$ uniform excitation. The laser powers were adjusted to give approximately equal incident photon fluxes on the CQW sample.

In the above PL imaging experiments, the PL from Xs in the QWs (X PL) was shadowed or overwhelmed by PL from the $\mathrm{n}^{+}$-GaAs layers $\left(\mathrm{n}^{+}-\mathrm{PL}\right)$ (see, for example, 
spectra in Figure 4.1). It is more revealing to examine X PL and $\mathrm{n}^{+}$-PL separately. We thus measured the $V_{b}$-dependent PL spectra at a locality (diameter $\phi \approx 3 \mu \mathrm{m}$ ) far from any 'traps' or 'local PL centers' under the same uniform excitation. The $P_{\text {laser }}$ 's of the HeNe and LD690 were lowered but still adjusted to give approximately equal incident photon fluxes.

The resultant $V_{b}$-dependent PL spectra are shown in Figure 7.15A and B for HeNe and LD690 excitation, respectively. The indirect-X PL lines for LD690 are not as narrow as those for HeNe, and the direct- to indirect-X transition is also not as smooth. We will leave the causes of these effects for future study, and maintain our focus on a comparison of the $V_{b}$-dependent spectrally integrated X-PL and $\mathrm{n}^{+}$-PL, whose spectral integrating range are defined by the white dashed line and arrows shown in Figure 7.15.
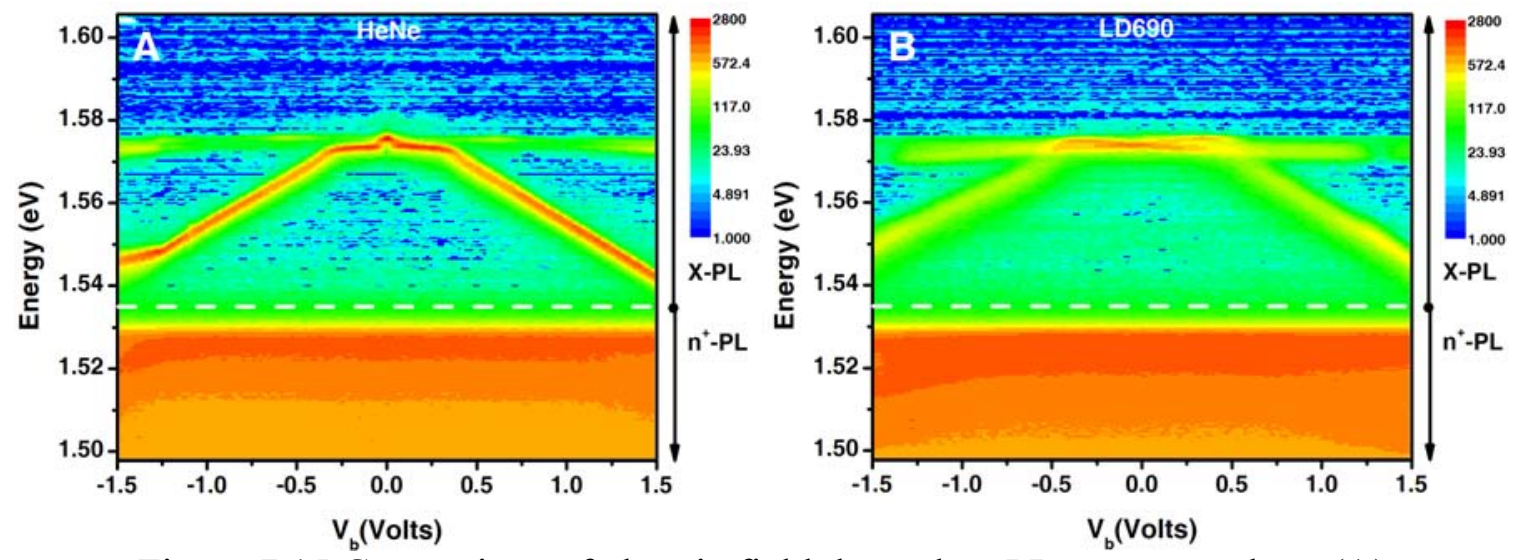

Figure 7.15 Comparison of electric field dependent PL spectra under a (A) $\mathrm{HeNe}(\mathrm{h} v=1.959 \mathrm{eV})$ and $(\mathbf{B})$ laser diode $(\mathrm{LD} 690, \mathrm{~h} v=1.802 \mathrm{eV})$ uniform excitation. The laser powers were adjusted to give approximately equal incident photon fluxes on the CQW sample.

The ratio of the $\mathrm{n}^{+}-\mathrm{PL}: \mathrm{HeNe}$ and $\mathrm{n}^{+}-\mathrm{PL}: \mathrm{LD} 690$ at $V_{b}=0$ is approximately 0.90 , which is close to the photon flux ratio, which is approximately 0.86 (Figure 7.16A and B). This confirms the accuracy of relative power measurements and the linear performance of the optical collection and detection system. 
For the case of LD690, the X-PL is quenched whereas $n^{+}-\mathrm{PL}$ is enhanced with increasing $\left|V_{b}\right|$ (Figure 7.16C and D). This can be attributed to carriers tunneled through $[127,128]$ or swept out of the CQW and the increasing significance of nonradiative recombination due to a longer indirect-X lifetime under applied electric fields. The photocurrents (or leakage current) are about two orders of magnitude lower than those obtained under HeNe excitation. The difference is more significant for $V_{b}>0.5 \mathrm{~V}$, where the main contribution of the photocurrent under LD690 excitation is due to the swept-out photoexcited carriers.

For the case of HeNe laser excitation, the initial PL quenching is pronounced, which is likely caused by a dramatic increase in the sweep-out of carriers photoexcited in the AlGaAs layers. The most important features are: (i) the parallel dependence of the photocurrent and photoluminescence (X-PL and $\mathrm{n}^{+}-\mathrm{PL}$ ) on $V_{b}$ (ii) the dramatic increase of both X-PL and $\mathrm{n}^{+}$-PL for $V_{b}$ less than about $-1.2 \mathrm{~V}$. By conservation of energy, we thus conclude that carrier re-injection is significant in the CQW sample, especially for $\mathrm{HeNe}$ laser excitation and a large negative bias $V_{b}$. Because the DPL emissions (see Sec. 7.1) were observed under these conditions, the unusual DPL emissions are likely associated with re-injected carriers rather than carriers or Xs photogenerated directly by laser excitation. If this is the case, DPL is similar to electroluminescence in light emission diodes (LEDs), which have clearly designed $\mathrm{p}-\mathrm{n}$ junctions. Though the numerous experimental results presented so far have suggested that the CQW sample exhibits asymmetry in the z-direction, the sample is unlikely to possess a clear-cut $p$-n junction because no p-type doping was introduced purposely in the growing process. Currently, the mechanisms of such an injection (i.e. possible double injection of both electrons and 
holes) are not clear. Nevertheless, experimental results suggest such an injection must be present.
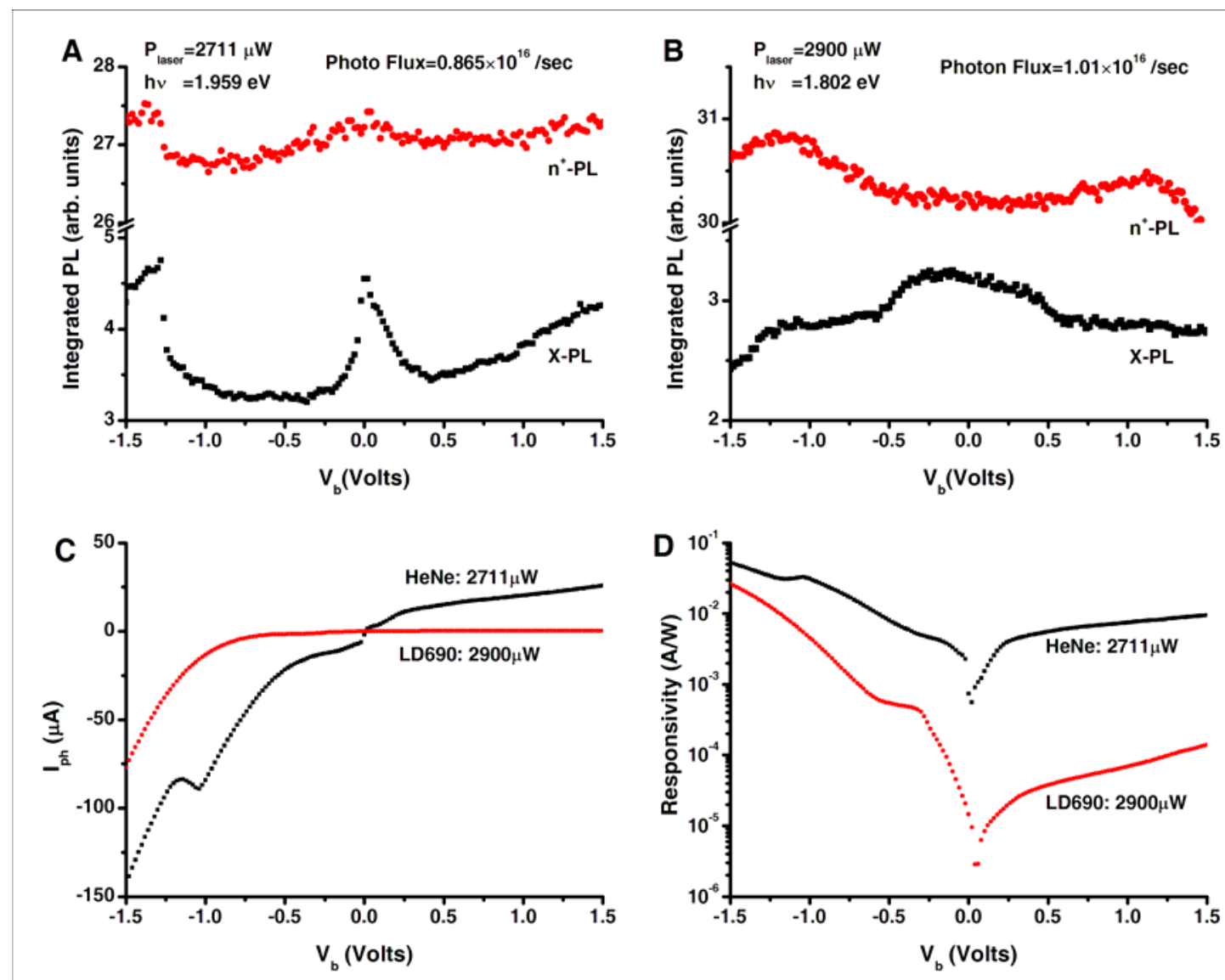

Figure 7.16 Comparisons of the PL- and photocurrent-voltage characteristics under $\mathrm{HeNe}(\mathrm{hv}=1.959 \mathrm{eV})$ and laser diode (LD690, $\mathrm{h} v=1.802 \mathrm{eV}$ ) uniform excitation.

\subsubsection{Confined spatially indirect exciton systems}

We have described the time-resolved photoluminescence (TRPL) measurements of the confined indirect-X systems in Sec. 5.4.3. The measured TRPL exhibits a typical long (up to $\sim 10 \mu \mathrm{s}$ ) and oscillatory decay, which we have attributed to carrier injection or space-charge buildup. In this section, we further compare the TRPL of these confined indirect-X systems with the transient photocurrent measured at $V_{b}=-0.95 \mathrm{~V}$, where 
extensive diagrams as a function of $T$ and $P_{\text {laser }}$ have been carried out and detailed in Chapter 5.
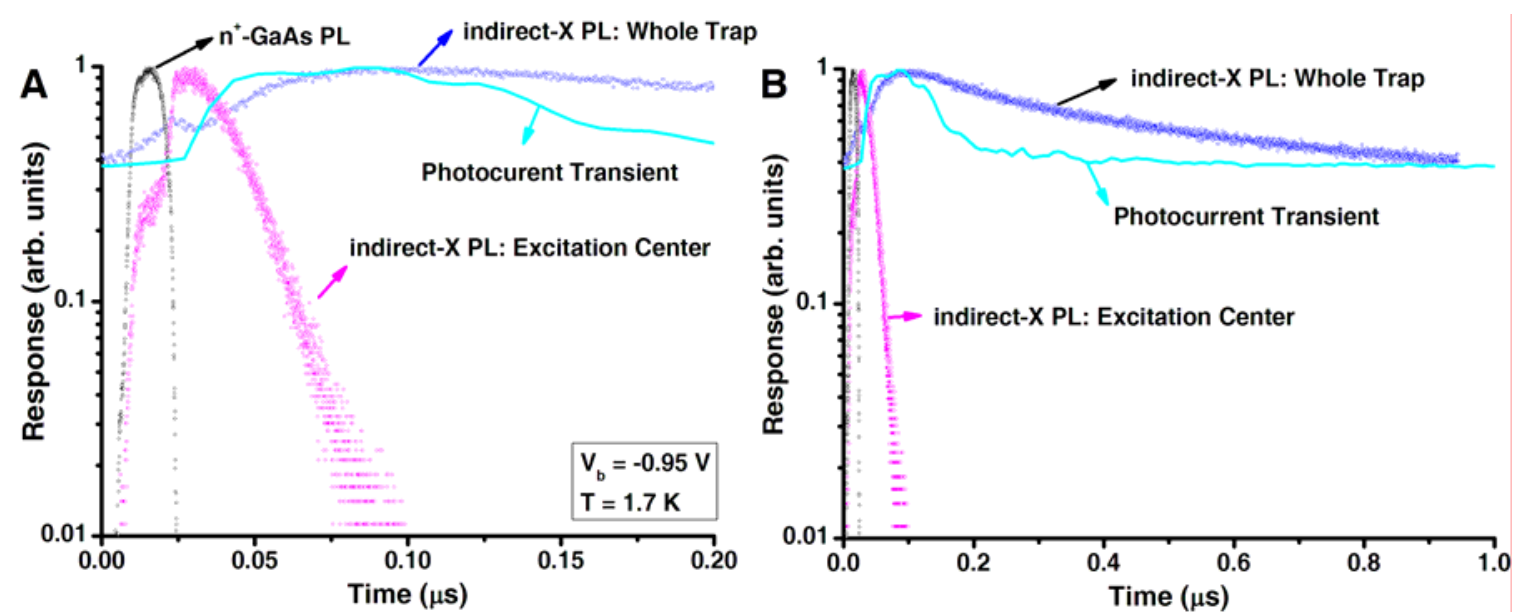

Figure 7.17 TRPL of indirect-Xs (blue dots) from Trap-A versus the transient photocurrent (cyan line) at $T=1.7 \mathrm{~K}$ and $V_{b}=-0.95 \mathrm{~V}$ (Laser diode: repetition rate $=100 \mathrm{kHz}$, average $P_{\text {laser }} \approx 24 \mu \mathrm{W}$, and the distance to Trap-A $\Delta y \approx 100 \mu \mathrm{m}$ for indirect-X TRPL; $\Delta \mathrm{y} \approx 0$ for the transient photocurrent). The TRPL of indirect-Xs (magenta dots) and $\mathrm{n}^{+}-\mathrm{GaAs}$ substrate layers (black dots) at the laser excitation center are also shown for comparison (Laser diode: repetition rate $=1 \mathrm{MHz}$, average $P_{\text {laser }} \approx 0.43$ $\mu \mathrm{W}$, and $\Delta \mathrm{y} \approx 100 \mu \mathrm{m}$ ). (A) Time scale: 0 to $0.2 \mu \mathrm{s}$. (B) Time scale: 0 to $1.0 \mu \mathrm{s}$.

TRPL of indirect-Xs from Trap-A (cf. Chapter 5) and the transient photocurrent at $T=1.7 \mathrm{~K}$ and $V_{b}=-0.95 \mathrm{~V}$ are shown in Figure 7.17 . The cyan curve represents the transient photocurrent under a pulsed laser excitation centered on Trap-A. Note that similar photocurrent transients were observed for excitation anywhere on the mesa at $V_{b}$ $=-0.95 \mathrm{~V}$. The blue dots are the TRPL of indirect-Xs within Trap-A under a similar pulsed excitation but $\sim 100 \mu \mathrm{m}$ away from Trap-A. TRPL of indirect-Xs at the laser excitation center decay much faster (decay time $\approx 6.5 \mathrm{~ns}-$ lifetime of indirect-Xs at $V_{b}=$ $-0.95 \mathrm{~V})$ as expected; however, TRPL of indirect-Xs from Trap-A follows the transient photocurrent up to $\sim 10 \mu \mathrm{s}$, which indicates a continuous generation of indirect-Xs at 
these 'traps'. These results suggest a strong correlation between the persistent photocurrent and the long indirect-X PL decay observed at 'traps'.

\subsection{Interpretations}

\subsubsection{Confined exciton systems}

We propose the following model for formation of indirect-Xs within confined small areas: The localized dark spot at the center of the X-cloud is associated with a pinhole which funnels a current filament through the $n^{+}-i-n^{+}$structure. This source of current is smaller than or on the order of our spatial resolution of about $2 \mu \mathrm{m}$. In the absence of photoexcitation, it contributes only to the leakage current, but provides a localized source of majority carriers of one type. For low excitation density, corresponding to regime-I (Figure 5.6), carriers of the opposite type flow toward the current source. Indirect-Xs are formed where the two types of carriers merge, resulting in an extended indirect-X system. Currently, models based on the carrier imbalance mechanism and in-plane carrier diffusion have been proposed [182,181]. However, formation of these indirect-X systems involves both cross-well and in-plane carrier transport as we have discussed previously (cf. Sec. 5.4 and Sec. 7.2). A full model taking into account of cross-well carrier transport and the presence of space charges is necessary. As the photoexcitation increases, at some point both types of carriers are injected into the CQW, resulting in an $\mathrm{X}$ density strongly peaked at the center (Figure 5.6B and Figure 5.7B) and a concentrated cloud as shown in the central image of Figure 5.2B. This corresponds to regime-II. As the excitation density increases further to reach regime-III, effects of the underlying Fermi statistics become apparent. The picture that has emerged is quite different from that of a condensation of Xs preexisting in the structure, which are photogenerated directly by laser excitation. 


\subsubsection{In-depth simulations}

In this section, we discuss briefly requirements for further in-depth simulations for the observed unusual DPL emission.

In general, the confining potential profile of quantum wells and barriers (i.e. the band diagram) is reciprocally influenced by the nonequilibrium charge transport through the structure, including charge accumulation, trapping, or depletion. The space charges provide a feedback to the charge carrier distribution that couples to the electric potential governing the transport according to Poisson's equation. The effect is more pronounced in semiconductor heterostructures with band discontinuities forming potential wells and barriers. The local space charge accumulation, nonlinear cross-well charge transport, and real-space carrier transport have provided mechanisms for negative differential resistance (NDR), current bistability, and nonlinear dynamics [199,200,193], many of which have been observed in the CQW sample studied.

Unlike the space-charge dynamics discussed in [199,200,193], the observed NDR regions exist only with proper combinations of the photoexcitation intensity $\left(P_{\text {laser }}\right)$, energy $(h v)$ and bias voltage $\left(V_{b}\right)$. The nature of these unusual I-V characteristics of the CQW sample studied has not been fully determined. The stationary and transient photocurrent measurements with various excitation energies and intensities suggest space charge accumulations, which give rise to the carrier injection or localized filament formation [201]. Many unusual phenomena observed in this CQW sample involve complicate processes. To simulate the formation processes will require a proper set of equations accounting for space charges, carrier injection, generation and recombination, and transport of the photogenerated carrier. To simulate all of these effects is a challenging task. 
A full theoretical description of charge/carrier transport in semiconductor structures requires a self-consistent solution of the coupled Maxwell's equations for the fields and a proper set of continuity equations for the transport and recombination of the carriers. The presence of space charges couples these sets of equations nonlinearly. For example, in thermodynamics equilibrium, the band-bending effect due to space charges is determined by a self-consistent solution of the coupled Schrödinger equation and Poisson's equation. The electrostatic potential in Schrödinger equation is calculated from Poisson's equation for a given carrier density distribution, and conversely, the carrier density is determined by the wave function calculated from the Schrödinger equation [202]. When a bias voltage is applied to the structure, a similar procedure is used and the transport equations must be solved simultaneously with Poisson's equation under nonequilibrium conditions. In time-dependent nonequilibrium situations, the finite dielectric or carrier relation time due to build-up or sweep-out of space charges can complicate the space-charge dynamics and make the problem intractable without proper approximation [203,201]. Therefore, from an experimentalist's point of view, a more pragmatic approach might be to sort out the physical processes involved experimentally with better controlled quantum well structures.

\subsection{Summary}

We have investigated the electric field dependent PL and photocurrent characteristics of the CQW samples for different excitation conditions: (i) various excitation energy (hv), (ii) different excitation spot sizes - uniform or localized $(\approx 5-10$ $\mu \mathrm{m}$ diameter spot), and (iii) continuous and pulsed excitation. Distant photoluminescence 
disappeared sharply when $\mathrm{h} v$ was tuned below the AlGaAs bandgap $\mathrm{E}_{\mathrm{gb}} \approx 1.94 \mathrm{eV}$ or above $\mathrm{E}_{\mathrm{ub}} \approx 2.34 \mathrm{eV} \mathrm{E} \mathrm{gb}$, which indicated the importance of the photocarriers generated in AlGaAs layers.

In addition, the photocurrent-voltage characteristics of the sample show asymmetry with an $\mathrm{N}$-shape negative differential resistance (NDR) regime and photocurrent gain, which signaled trapping of photogenerated carriers and/or buildup of space charges. Therefore, both (a) generation and transport of carrier in/cross the AlGaAs barriers and (b) carrier capture and escape from QWs are of importance for the formation of the observed localized dense cold X systems and PL ring patterns.

With respect to the formation of the confined indirect-X systems ('traps'), we have ruled out the QW thickness fluctuation as a cause of X collection and demonstrated that these traps are electrically active. We also compared the long oscillatory PL decay with the transient photocurrent measurements, which confirmed the vital role of the photo-assisted carrier transport or injection across the structure.

We thus concluded that the observed degenerate X-systems in the 'traps' and extended ring patterns form through the photo-induced carrier injection, trapping, transport and space charge buildup. Finally, possibility of developing full models was discussed. 


\section{Chapter 8 Conclusion}

\subsection{To BEC or not to BEC?}

Over the past thirty years, there have been numerous claims of the observation of Bose-Einstein condensation or superfluidity of excitons. For example, there were early claims in $\mathrm{CuCl}$ [204-206], $\mathrm{Cu}_{2} \mathrm{O}$ [207-209], and exciton-polaritons in microcavities [210]. These were followed by much debate and many retractions [211-213]. The primary problem is that the experimental claims have been subject to multiple interpretations. To provide details of all the claims and counter claims would be a formidable task, which we shall not pursue. We shall only briefly review some such claims and progress in the fabrication of suitable CQW, which is the system studied in this dissertation.

The first set of experiments claiming observation of Bose statistics based on temperature dependent photoluminescence (PL) linewidth in CQW [36] encountered difficulties with trapped excitons due to interface disorder [214-216]. More recently, there have been several claims of condensation of indirect excitons in coupled quantum wells $[217,134,46,218,219]$. The work based on nonlinear PL dynamics by Butov et al. claimed stimulated bosonic scattering of excitons in highly statistically degenerate regimes $[37,133,134]$. However, spatially- and time-resolved PL measurements on the same CQW sample have shown that the reported nonlinear PL dynamics is distorted by the presence of scattered PL from the substrate $n^{+}$-GaAs layers (see Sec. 4.4). Recently observations of distant PL rings with an extended dark region between the ring and 
excitation spot (see Chapter 6 ) were reported by two groups [46,47]. These were initially attributed to superfluid dark excitons or a macroscopically ordered exciton state with coherence. The current consensus is that the Xs are formed close to where the PL rings are observed. The formation mechanisms of these PL rings are attributed to charge imbalance and in-plane charge transport in ref. [181,182]. However, we have demonstrated in this dissertation that the cross-well carrier transport and capture are vital to any interpretation. The exact formation mechanisms are worth further investigations. The fragmented PL rings were interpreted as evidence of macroscopic ordered exciton states in ref. [46,181]. However, the experimental observation was of ordered fragmented PL patterns, which are not necessarily associated with an ordered exciton collective state arising from the intrinsic exciton interaction or macroscopic coherence. For example, excitons in the PL rings could be formed through inhomogeneous carrier injection, which exhibit fragmented patterns due to the presence of space charge. We have also reported an extensive phase diagram of dense cold excitons confined in localities [44,45]. As detailed in Chapter 5, depending on the exciton density and temperature these confined exciton systems exhibit interesting distinct phases (states), but there is no clear evidence for BEC. Spatially indirect-Xs in the studied CQW samples exhibit many interesting physical phenomena, but may be not necessarily related to BEC or superfluidity of excitons.

Many of the previously claimed observations of excitonic BEC involved either exciton-polariton induced lasing [210] or some type of resonant pumping, or relied on spectral narrowing of excitonic luminescence $[36,218]$ or fast expansion of excitons [207]. These are subject to plausible alternative interpretations without resorting to Bose- 
Einstein condensation. More decisive experimental evidence, such as the demonstration of the coherence of the state, is required to firmly establish BEC or any quantum collective effects of excitons in these solid-state systems.

\subsection{Summary}

Spatially indirect-Xs in CQW are composed of pairs of electron and hole confined in separate QWs. The energy and lifetime of indirect-X are tunable by electric fields; while the effective mass can be manipulated by magnetic fields. Indirect-Xs exhibit a long lifetime, high cooling/thermalization rate, and a stable ground state due to the dipolar repulsive interaction - promising attributes for the observation of collective quantum effects. These properties of indirect-Xs were investigated through timeintegrated or time-resolved PL spectroscopy as a function of temperature (T), bias voltage $\left(V_{b}\right)$ (electric field), laser excitation intensity $\left(P_{\text {laser }}\right)$, and magnetic field $(B)$.

Degenerate exciton systems have been produced in quasi-two-dimensional confined areas in semiconductor coupled quantum well structures. We observed contractions of clouds containing tens of thousands of excitons within areas as small as $(10 \mu \mathrm{m})^{2}$ near 10 Kelvin. The spatial and energy distributions of optically active excitons were determined by measuring photoluminescence as a function of temperature and laser excitation and were used as thermodynamic quantities to construct the phase diagram of the exciton system, which demonstrates the existence of distinct phases. Spatially and time resolved PL spectroscopy were also used to study the formation mechanisms of

these X systems. TRPL exhibited extremely long ( $\mu$ s) oscillatory decays, which implied continuous formation of indirect-Xs through photo-induced carrier transport and injection, and a buildup of space charges. 
Exploring the phase space with various parameters, we have found that the CQW studied exhibited many unusual phenomena. We reviewed briefly the fragmented ringpatter PL emissions observed hundreds of microns away from a localized $\mathrm{HeNe}$ laser excitation spot $[46,47]$ and other local PL centers. The ordered distant ring-pattern PL signaled macroscopic carrier transport and possible ordering in the system.

The indirect-X cloud confined in small areas, distant ring-pattern PL emissions, and local PL centers all disappeared when excitation energy hv was tuned below the AlGaAs bandgap $\mathrm{E}_{\mathrm{gb}} \approx 1.94 \mathrm{eV}$ or above $\mathrm{E}_{\mathrm{ub}} \approx 2.34 \mathrm{eV}$, which indicated that these phenomena were likely caused by the carriers photogenerated in the AlGaAs layers. In addition, the photocurrent-voltage characteristics of the sample showed asymmetry with an N-shape negative differential resistance (NDR) regime and photocurrent gain, which signaled trapping of photogenerated carriers and/or buildup of space charges. Therefore, both (a) generation and transport of carrier in and across the AlGaAs barriers and (b) carrier capture and escape from QWs are important for formation of the observed localized dense cold X systems and PL ring patterns.

We thus concluded that the observed degenerate X-systems in the small areas and ring-pattern PL emissions are formed through the photo-induced carrier injection, trapping, transport and space charge buildup. These insights into the formation mechanisms of the observed cold exciton systems may lead to new strategies for producing confined cold X systems artificially in a more controllable way.

\subsection{Future directions}

Optically properties of dense exciton systems in quantum wells have been studied extensively both experimentally and theoretically. Traditional photoluminescence 
spectroscopy, which is a simple and valuable means by which to study the nature of the lowest excitation states, cannot yield much information about the coherence of the exciton systems which is vital for establishing excitonic BEC. A superfluid or BEC transition of the exciton system might manifest itself as a sharp narrowing of the PL peak at the critical temperature or number density. However, the intrinsic exciton PL in the quantum well systems would be masked by the inhomogeneous broadening due to the inevitable disorder. Therefore the narrowing of PL linewidth shown in Chapter 4 and Chapter 5 is not unambiguous evidence of collective quantum effects. It is certainly worth investigating the higher order coherence $[220,221]$ of photoluminescence or superradiance from these cold exciton systems [222-226] by the Hanbury Brown-Twiss or photon bunching experiments [227]. The spatial coherence of emissions from different locations of the system can also be measured by two-field correlation or optical homodyne tomography [228,229]. These results could clarify if the indirect-X photoluminescence possesses quantum coherent properties related to a collective exciton state. Other linear optical techniques such as resonant Rayleigh scattering $[230,231]$ and nonlinear optical techniques such as four-wave mixing [232-234] can reveal the spatial or temporal coherence properties of the exciton states as well. Stimulated light backscattering and other nonlinear optical phenomena from exciton Bose-Einstein condensates have also been predicted theoretically $[235,236]$. The experimental search for exciton $\mathrm{BEC}$ has been historically controversial due to the strong interacting nature of excitons and the lack of unambiguous evidence of the macroscopic coherence. To establish excitonic BEC with optical techniques, it is vital to investigate effects that can be associated with the optical manifestation of exciton coherent collective states. 
Many attempts to obtain dense exciton systems are with the motive of realizing and excitonic BEC in solid-state systems. However, the exciton or electron-hole system by itself is interesting for the observation of many fascinating physical phenomena, such as the rich phenomena observed in coupled quantum wells. However, the leakage current is significantly higher in a biased $n-i-n$ structure, such as the one studied, than in a reversed-biased $p-i-n$ structure. The leakage current could affect the photoexcited carrier relaxation dynamics and other processes significantly. The experimental results reported in this dissertation have indicated the decisive role of carrier transport, capture, and injection in the formation of the observed ring PL patterns and exciton systems confined in small areas. The intrinsic exciton phenomena need to be distinguishable from other phenomena, such as the space-charge effects or carrier transport and injection. This can be achieved by using less leaky samples and a pico-second ultrafast laser excitation in resonance with the direct exciton transitions. Secondly, it would be interesting to investigate the possibility of artificially generating cold, dense and long-lived indirect-X systems by tunneling injection mechanisms [237,238]. This would provide new possibilities for exploration of collective quantum phenomena of excitons in solid-state systems. 


\section{Appendix}

\section{A. List of Symbols}

$\begin{array}{ll}\mathrm{a}_{\mathrm{x}} & \text { Bohr radius of excitons } \\ B & \text { Magnetic field } \\ \mathrm{c} & \text { Velocity of light } \\ \mathrm{E}_{0} & \text { Peak energy of an exciton PL line } \\ \mathrm{E}_{\mathrm{c}(\mathrm{v})} & \text { Energy of conduction (valence) band edge } \\ \Delta \mathrm{E}_{\mathrm{c}(\mathrm{v})} & \text { Conduction (valence) band offset } \\ \mathrm{E}_{\mathrm{b}} & \text { Exciton binding energies } \\ \mathrm{E}_{\mathrm{F}} & \text { Fermi level in semiconductor } \\ \mathrm{E}_{\mathrm{g}} & \text { Bandgap of semiconductor } \\ \mathrm{E}_{\mathrm{X}} & \text { Mean energy of an exciton PL line } \\ \mathrm{F} & \text { Electric field } \\ \mathrm{G} & \text { Generation rate of carriers } \\ \mathrm{h}, \hbar & \text { Plank's constant and reduced Plank's constant } \\ \mathrm{I}_{\mathrm{pc}} & \text { Photo-Current } \\ \mathrm{I}_{\mathrm{SC}} & \text { Short-circuit current of solar cell } \\ \mathrm{I}_{\text {laser }} & \text { Laser light intensity } \\ \mathrm{I}_{\mathrm{PL}} & \text { Spatially integrated photoluminescence image intensity } \\ \mathrm{I}_{\mathrm{X}} & \text { Spectrally integrated intensity of an exciton PL line } \\ \mathrm{k}_{\mathrm{B}} & \text { Boltzmann's constant } \\ \mathrm{L}_{\mathrm{e}(\mathrm{h})} & \text { Diffusion length for electron (hole) } \\ \mathrm{m}_{\mathrm{e}(\mathrm{h})} & \text { Electron (hole) effective mass } \\ \mathrm{m}_{0} & \text { Rest mass of electron } \\ \mathrm{I}_{0} & \text { Photoluminescence peak intensity } \\ P_{l a s e r} & \text { Incident optical or laser power right before the front surface of the device } \\ \mathrm{q} & \text { Electronic charge } \\ \mathfrak{R} & \text { Responsivity of detector } \\ \mathrm{R} & \text { Reflectivity } \\ \mathrm{t} & \text { Time } \\ \mathrm{t}_{\mathrm{tr}} & \text { Carrier transit time } \\ T & \text { Temperature } \\ V_{b} & \text { Applied bias voltage } \\ \mathrm{V}_{\mathrm{OC}} & \text { Open circuit voltage of solar cell } \\ \alpha & \text { Absorption coefficient } \\ \Gamma & \text { Internal, external quantum efficiency } \\ \eta_{\mathrm{i}}, \eta_{\mathrm{ext}} & \end{array}$


$\eta_{\mathrm{r}} \quad$ Radiative recombination quantum efficiency

$\lambda \quad$ Wavelength of light

$\mu_{\mathrm{e}(\mathrm{h}) \quad \text { Mobility of electrons (holes) }}$

$v \quad$ Frequency of light

$\sigma_{\mathrm{r}} \quad$ Root mean square radius of a laser excitation spot

$\sigma_{\mathrm{PL}} \quad$ Root mean square radius of photoluminescence images

$\tau, \tau_{\mathrm{r}}, \tau_{\mathrm{nr}} \quad$ Lifetime, radiative lifetime, nonradiative lifetime

$\tau_{\mathrm{e}(\mathrm{h})}$

Electron (hole) recombination lifetime 


\section{B. Sample Preparation}

The processing involves two photolithographic steps to define (i) mesa and (ii) metal contact layers. We started with a cleaved piece of sample $\sim 12 \times 10 \mathrm{~mm}^{2}$ in size, which will yield $\sim 6$ chips in the end. First, after defining the mesa layer with standard lithographic procedure, the sample was wet etched down to the bottom $\mathrm{n}^{+}$-GaAs gate layer. Then the photoresist is stripped off and the sample cleaned with hot acetone. Next, the gate metal contact layer was defined again with a standard lithographic procedure. The sample was then deposited with $\mathrm{Ge} / \mathrm{Pd} / \mathrm{Au}\left(250 / 200 / 2000 \mathrm{~A}^{\circ}\right)$ alloy layers with an ebeam evaporator. Top and bottom gates are defined after the lift off step. The processed piece was further cleaved along the (110) planes into separate chips (chip area $\sim 3.5 \times 4.5$ $\mathrm{mm}^{2}$ ) for rapid thermal annealing at $\sim 320-360^{\circ} \mathrm{C}$ for $\sim 60$ seconds with ambient $\mathrm{Ag}$ and $\mathrm{H}_{2}$ gases to form ohmic contacts. The chip was finally bond to a proper open cavity die for gold wire bonding.

Below are the machines and chemicals used for sample processing in the Microlab at UC, Berkeley:

Machines Used:

- Photolithography: $\sim 1 \mu \mathrm{m}$ resolution is sufficient

Mask maker: Emulsion masks for positive photoresists.

* Photoresist spinner: Headway Photoresist Spinner

- Mask Aligner: Quintel Mask Aligner ( $\times 1)$ or Canon 4× Projection Mask Aligner

- Testing/inspection

* Surface Profiler: Alpha-Step IQ Surface Profiler

- Thin film deposition

* E-beam evaporator: ULTEK e-beam evaporator

- Annealing 
* Rapid thermal annealing: Heatpulse 210T RTA system

- Packaging

* Bonding machine: Westbond Model 7400B Ultra Sonic Bonders

\section{Chemicals used:}

* Photolighography

- Photoresist: Shipley S1818 positive photoresist

- Developer: Shipley 452 developer

- Stripper: J.T. Baker PRS-3000 or Acetone

* Etching

- GaAs wet etching: $\mathrm{H}_{3} \mathrm{PO}_{4}: \mathrm{H}_{2} \mathrm{O}_{2}: \mathrm{H}_{2} \mathrm{O}=3: 1: 25 \rightarrow \sim 0.30 \mu \mathrm{m} /$ minute etch rate. 


\section{References}

1. $\quad$ S. Bose, "Plancks gesetz und lichtquantenhypothese," Z. Phys. 26, 178 (1924).

2. A. Einstein, "Quantentheorie des einatomigen idealen gases: Zweite abhandlung," Sitzungber. Preuss. Akad. Wiss. 1925, 3 (1925).

3. R. C. Majumdar \& S. Bose, "Bosons, presented to satyendra nath bose on the occasion of his seventieth birthday," (Hindustan Publ. Corp. (India), Delhi,, 1964).

4. $\quad$ F. London, "On the bose-einstein condensation," Phys. Rev. 54, 947 (1938).

5. M. H. Anderson, J. R. Ensher, M. R. Matthews, C. E. Wieman \& E. A. Cornell, "Observation of bose-einstein condensation in a dilute atomic vapor," Science 269, 198 (1995).

6. K. B. Davis, M. O. Mewes, M. R. Andrews et al., "Bose-einstein condensation in a gas of sodium atoms," Phys. Rev. Lett. 75, 3969 (1995).

7. E. A. Cornell \& C. E. Wieman, "Nobel lecture: Bose-einstein condensation in a dilute gas, the first 70 years and some recent experiments," Rev. Mod. Phys. 74, 875 (2002).

8. W. Ketterle, "Nobel lecture: When atoms behave as waves: Bose-einstein condensation and the atom laser," Rev. Mod. Phys. 74, 1131 (2002).

9. C. Pethick \& H. Smith, "Bose-einstein condensation in dilute gases," (Cambridge University Press, Cambridge ; New York, 2002).

10. L. P. Pitaevskiæi \& S. Stringari, "Bose-einstein condensation," (Clarendon Press, Oxford, 2003).

11. S. A. Moskalenko, "Reversible optico-hydrodynamic phenomena in a nonideal exciton gas," Sov Phys-Sol State 4, 199 (1962).

12. J. M. Blatt, W. Brandt \& K. W. Boer, "Bose-einstein condensation of excitons," Phys. Rev. 126, 1691 (1962).

13. L. V. Keldysh \& Y. V. Kopaev, "Possible instability of semimetallic state toward coulomb interaction," Sov. Phys. Solid State 6, 2219 (1965).

14. L. V. Keldysh \& A. N. Kozlov, "Collective properties of excitons in semiconductors," Sov. Phys. JETP 27, 521 (1968).

15. L. V. Keldysh, "Macroscopic coherent states of excitons in semiconductors," in Bose-einstein condensation 246 (Cambridge University Press, Cambridge ; New York, 1995).

16. E. Hanamura \& H. Haug, "Condensation effects of excitons," Phys. Rep.-Rev. Sec. Phys. Lett. 33, 209 (1977).

17. C. Comte \& P. Nozieres, "Exciton bose condensation - the ground-state of an electron-hole gas .1. Mean field description of a simplified model," J Phys-Paris 43, 1069 (1982). 
18. P. Nozieres \& C. Comte, "Exciton bose condensation - the ground-state of an electron-hole gas .2. Spin states, screening and band-structure effects," J PhysParis 43, 1083 (1982).

19. X. J. Zhu, P. B. Littlewood, M. S. Hybertsen \& T. M. Rice, "Exciton condensate in semiconductor quantum-well structures," Phys. Rev. Lett. 74, 1633 (1995).

20. I. E. Perakis, "Condensed-matter physics - exciton developments," Nature 417, 33 (2002).

21. G. E. Bauer \& T. Ando, "Theory of band-gap renormalization in modulationdoped quantum wells," J. Phys. C 19, 1537 (1986).

22. C. Delalande, "Semiconductor quantum wells," in Quantum optics of confined systems 181 (Kluwer Academic Publishers, Dordrecht ; Boston, 1996).

23. M. Combescot \& C. Tanguy, "New criteria for bosonic behavior of excitons," Europhysics Letters 55, 390 (2001).

24. R. Zimmermann, K. Kilimann, W. D. Kraeft, O. Kremp \& G. Ropke, "Dynamical screening and self-energy of excitons in electron-hole plasma," Physica Status Solidi B-Basic Research 90, 175 (1978).

25. G. Ropke, T. Seifert, H. Stolz \& R. Zimmermann, "Influence of exciton gas and electron-hole plasma on exciton energy levels," Physica Status Solidi B-Basic Research 100, 215 (1980).

26. H. Stolz, R. Zimmermann \& G. Ropke, "Correlated hartree-fock theory of the electron-hole plasma containing exciton bound states," Physica Status Solidi BBasic Research 105, 585 (1981).

27. H. Haug \& S. Schmittrink, "Electron theory of the optical-properties of laserexcited semiconductors," Prog. Quant. Electron. 9, 3 (1984).

28. J. Fernandez-Rossier, C. Tejedor, L. Munoz \& L. Vina, "Polarized interacting exciton gas in quantum wells and bulk semiconductors," Phys. Rev. B 54, 11582 (1996).

29. C. Ciuti, V. Savona, C. Piermarocchi, A. Quattropani \& P. Schwendimann, "Role of the exchange of carriers in elastic exciton-exciton scattering in quantum wells," Phys. Rev. B 58, 7926 (1998).

30. G. Rochat, C. Ciuti, V. Savona et al., "Excitonic bloch equations for a twodimensional system of interacting excitons," Phys. Rev. B 61, 13856 (2000).

31. C. F. Klingshirn, "The electron-hole plasma," in Semiconductor optics 306 (Springer, Berlin ; New York, 1997).

32. C. D. Jeffries \& L. V. Keldysh (eds.) Electron-hole droplets in semiconductors (North-Holland, Amsterdam, 1983).

33. T. M. Rice, "The electron-hole liquid in semiconductors: Theoretical aspects," in Solid state physics - advances in research and applications 32, 1 (Academic Press, New York, 1977).

34. R. Cingolani \& K. Ploog, "Frequency and density dependent radiative recombination processes in iii-v semiconductor quantum-wells and superlattices," Adv. Phys. 40, 535 (1991).

35. S. A. e. Moskalenko \& D. W. Snoke, "Bose-einstein condensation of excitons and biexcitons : And coherent nonlinear optics with excitons," (Cambridge University Press, Cambridge, UK ; New York, 2000). 
36. T. Fukuzawa, E. E. Mendez \& J. M. Hong, "Phase-transition of an exciton system in gaas coupled quantum-wells," Phys. Rev. Lett. 64, 3066 (1990).

37. L. V. Butov, A. Imamoglu, A. V. Mintsev, K. L. Campman \& A. C. Gossard, "Photoluminescence kinetics of indirect excitons in gaas/alxga1-xas coupled quantum wells," Phys. Rev. B 59, 1625 (1999).

38. A. L. Ivanov, P. B. Littlewood \& H. Haug, "Bose-einstein statistics in thermalization and photoluminescence of quantum-well excitons," Phys. Rev. B 59, 5032 (1999).

39. A. L. Ivanov, "Quantum diffusion of dipole-oriented indirect excitons in coupled quantum wells," Europhys. Lett. 59, 586 (2002).

40. P. C. Hohenberg, "Existence of long-range order in one and two dimensions," Phys. Rev. 158, 383 (1967).

41. V. N. Popov, "On the theory of the superfluidity of two- and one- dimensional bose systems," Theor. Math. Phys. 11, 565 (1973).

42. V. Bagnato \& D. Kleppner, "Bose-einstein condensation in low-dimensional traps," Phys. Rev. A 44, 7439 (1991).

43. W. Ketterle \& N. J. van Druten, "Bose-einstein condensation of a finite number of particles trapped in one or three dimensions," Phys. Rev. A 54, 656 (1996).

44. L. V. Butov, C. W. Lai, A. L. Ivanov, A. C. Gossard \& D. S. Chemla, "Towards bose-einstein condensation of excitons in potential traps," Nature 417, 47 (2002).

45. C. W. Lai, J. Zoch, A. C. Gossard \& D. S. Chemla, "Phase diagram of degenerate exciton systems," Science 303 (2004).

46. L. V. Butov, A. C. Gossard \& D. S. Chemla, "Macroscopically ordered state in an exciton system," Nature 418, 751 (2002).

47. D. Snoke, S. Denev, Y. Liu, L. Pfeiffer \& K. West, "Long-range transport in excitonic dark states in coupled quantum wells," Nature 418, 754 (2002).

48. G. H. Wannier, "The structure of electronic excitation levels in insulating crystals," Phys. Rev. 52, 191 (1937).

49. R. S. Knox, "Theory of excitons," (Academic Press, New York, 1963).

50. S. Charbonneau, M. L. W. Thewalt, E. S. Koteles \& B. Elman, "Transformation of spatially direct to spatially indirect excitons in coupled double quantum wells," Phys. Rev. B 38, 6287 (1988).

51. S. Adachi, "Gaas and related materials: Bulk semiconducting and superlattice properties," (World Scientific, Singapore ; River Edge, NJ, 1994).

52. M. Cardona, "Band parameters of semiconductors with zincblende, wurtzite, and germanium structure," J. Phys. Chem. Solids 24, 1543 (1963).

53. J. R. Chelikowsky \& M. L. Cohen, "Nonlocal pseudopotential calculations for electronic structure of 11 diamond and zincblende semiconductors," Phys. Rev. B 14, 556 (1976).

54. B. I. Min, S. Massidda \& A. J. Freeman, "Structural and electronic properties of bulk gaas, bulk alas, and the gaas/alas superlattice," Phys. Rev. B 38, 1970 (1988).

55. J. R. Chelikowsky, T. J. Wagener, J. H. Weaver \& A. Jin, "Valence-band and conduction-band densities of states for tetrahedral semiconductors - theory and experiment," Phys. Rev. B 40, 9644 (1989). 
56. P. Y. Yu \& M. Cardona, "Fundamentals of semiconductors: Physics and materials properties," (Springer, Berlin ; New York, 2001).

57. S. L. Chuang, "Physics of optoelectronic devices," (Wiley, New York, 1995).

58. C. Kittel \& C. Y. Fong, "Quantum theory of solids," (Wiley, New York, 1987).

59. J. M. Luttinger, "Quantum theory of cyclotron resonance in semiconductors general theory," Phys. Rev. 102, 1030 (1956).

60. Z. Ikonic, V. Milanovic \& D. Tjapkin, "Valence subband structure of [100]grown, [110]-grown, and [111]-grown gaas-(al,ga)as quantum wells and the accuracy of the axial approximation," Phys. Rev. B 46, 4285 (1992).

61. Y. C. Chang \& J. N. Schulman, "Interband optical transitions in gaas-ga ${ }_{1-x} a l_{x} a s$ and inas-gasb superlattices," Phys. Rev. B 31, 2069 (1985).

62. J. J. Hopfield, "Theory of the contribution of excitons to the complex dielectic constant of crystals," Physical Review 112, 1555 (1958).

63. M. D. Sturge \& E. I. Rashba, "Excitons," (North-Holland Pub. Co., Amsterdam ; New York, 1982).

64. C. Y. P. Chao \& S. L. Chuang, "Analytical and numerical solutions for a twodimensional exciton in momentum space," Phys. Rev. B 43, 6530 (1991).

65. H. Haug \& S. W. Koch, "Quantum theory of the optical and electronic properties of semiconductors," (World Scientific, Singapore, 1994).

66. S. Schmitt-Rink, D. S. Chemla \& D. A. B. Miller, "Theory of transient excitonic optical nonlinearities in semiconductor quantum-well structures," Physical Review B 32, 6601 (1985).

67. L. C. Andreani \& A. Pasquarello, "Accurate theory of excitons in gaas/ga ${ }_{1-x} a l_{x} a s$ quantum wells," Phys. Rev. B 42, 8928 (1990).

68. R. Winkler, "Excitons and fundamental absorption in quantum wells," Phys. Rev. B 51, 14395 (1995).

69. L. V. Keldysh, "Excitons in semiconductor dielectric nanostructures," Physica Status Solidi A-Applied Research 164, 3 (1997).

70. C. Weisbuch \& B. Vinter, "Quantum semiconductor structures : Fundamentals and applications," (Academic Press, Boston, 1991).

71. G. Bastard, "Wave mechanics applied to semiconductor heterostructures," (Éditions de physique, Les Ulis, France, 1988).

72. D. A. B. Miller, D. S. Chemla, T. C. Damen et al., "Band-edge electroabsorption in quantum well structures - the quantum-confined stark-effect," Phys. Rev. Lett. 53, 2173 (1984).

73. T. H. Wood, C. A. Burrus, D. A. B. Miller et al., "High-speed optical modulation with gaas/gaalas quantum wells in a p-i-n-diode structure," Appl. Phys. Lett. 44, 16 (1984).

74. D. A. B. Miller, D. S. Chemla, T. C. Damen et al., "Electric-field dependence of optical-absorption near the band-gap of quantum-well structures," Phys. Rev. B 32, 1043 (1985).

75. L. Vina, E. E. Mendez, W. I. Wang, L. L. Chang \& L. Esaki, "Stark shifts in gaas/gaalas quantum-wells studied by photoluminescence spectroscopy," J. Phys. C 20, 2803 (1987).

76. S. Schmitt-Rink, D. S. Chemla \& D. A. B. Miller, "Linear and nonlinear opticalproperties of semiconductor quantum wells," Advances in Physics 38, 89 (1989). 
77. T. H. Wood, C. A. Burrus, D. A. B. Miller et al., "131 ps optical modulation in semiconductor multiple quantum wells (mqws)," Ieee Journal of Quantum Electronics 21, 117 (1985).

78. H. Kawai, J. Kaneko \& N. Watanabe, "Doublet state of resonantly coupled algaas gaas quantum wells grown by metalorganic chemical vapor deposition," Journal of Applied Physics 58, 1263 (1985).

79. A. Yariv, C. Lindsey \& U. Sivan, "Approximate analytic solution for electronic wave functions and energies in coupled quantum wells," Journal of Applied Physics 58, 3669 (1985).

80. E. J. Austin \& M. Jaros, "Carrier lifetimes and localization in coupled gaasgaalas quantum wells in high electric fields," Journal of Physics C-Solid State Physics 19, 533 (1986).

81. H. Q. Le, J. J. Zayhowski \& W. D. Goodhue, "Stark effect in $a_{x} g a_{1-x} a s$ gaas coupled quantum wells," Applied Physics Letters 50, 1518 (1987).

82. Y. J. Chen, E. S. Koteles, B. S. Elman \& C. A. Armiento, "Effect of electric fields on excitons in a coupled double quantum well structure," Physical Review B 36, 4562 (1987).

83. L. D. Landau \& E. M. Lifshiëtìs, "Quantum mechanics: Non-relativistic theory," (Pergamon Press, Oxford; New York, 1991).

84. J. H. Davies, "The physics of low-dimensional semiconductors: An introduction," (Cambridge University Press, Cambridge, U.K. ; New York, NY, USA, 1998).

85. T. Chakraborty \& P. Pietiläinen, "The quantum hall effects : Integral and fractional," (Springer-Verlag, Berlin ; New York, 1995).

86. S. D. Sarma \& A. Pinczuk, "Perspectives in quantum hall effects : Novel quantum liquids in low-dimensional semiconductor structures," (Wiley, New York, 1997).

87. Z. F. Ezawa, "Quantum hall effects : Field theoretical approach and related topics," (World Scientific, Singapore ; River Edge, NJ, 2000).

88. T. C. Damen, J. Shah, D. Y. Oberli et al., "Dynamics of exciton formation and relaxation in gaas quantum wells," Phys. Rev. B 42, 7434 (1990).

89. R. Ferreira, P. Rolland, P. Roussignol et al., "Time resolved exciton transfer in gaas/al $\mathrm{ga}_{1-\mathrm{x}}$ as double quantum-well structures," Phys. Rev. B 45, 11782 (1992).

90. R. Ferreira \& G. Bastard, "Tunneling and relaxation in semiconductor double quantum wells," Rep. Prog. Phys. 60, 345 (1997).

91. A. Alexandrou, J. A. Kash, E. E. Mendez et al., "Electric-field effects on exciton lifetimes in symmetrical coupled gaas-al ${ }_{0.3} g a_{0.7}$ as double quantum wells," Phys. Rev. B 42, 9225 (1990).

92. R. A. Kaindl, M. A. Carnahan, D. Hagele, R. Lovenich \& D. S. Chemla, "Ultrafast terahertz probes of transient conducting and insulating phases in an electron-hole gas," Nature 423, 734 (2003).

93. Y. E. Lozovik \& O. K. Berman, "Phase transitions in a system of two coupled quantum wells," JETP Lett. 64, 573 (1996).

94. Y. E. Lozovik \& O. L. Berman, "The excitonic superfluid liquid in the system of spatially separated electrons and holes," Phys. Scr. 55, 491 (1997).

95. Y. E. Lozovik, O. L. Berman \& V. G. Tsvetus, "Superfluidity of indirect magnetoexcitons in coupled quantum wells," JETP Lett. 66, 355 (1997). 
96. Y. E. Lozovik \& O. L. Berman, "Phase transitions in a system of spatially separated electrons and holes," J. Exp. Theor. Phys. 84, 1027 (1997).

97. Y. E. Lozovik, O. L. Berman \& M. Willander, "Superfluidity of indirect excitons and biexcitons in coupled quantum wells and superlattices," J. Phys.-Condes. Matter 14, 12457 (2002).

98. S. B. T. de-Leon \& B. Laikhtman, "Phase diagram of quasi two-dimensional electron-hole gas in single and coupled quantum wells," Physica Status Solidi aApplied Research 190, 689 (2002).

99. J. Feldmann, G. Peter, E. O. Gobel et al., "Linewidth dependence of radiative exciton lifetimes in quantum-wells," Phys. Rev. Lett. 59, 2337 (1987).

100. H. B. Bebb \& E. W. Williams, "Photoluminescence i: Theory," in Semiconductors and semimetals 8, 181 (Academic Press, New York, 1972).

101. K. K. Bajaj, "Use of excitons in materials characterization of semiconductor system," Mater. Sci. Eng. R-Rep. 34, 59 (2001).

102. L. Schultheis, A. Honold, J. Kuhl, K. Kohler \& C. W. Tu, "Optical dephasing of homogeneously broadened two-dimensional exciton-transitions in gaas quantumwells," Phys. Rev. B 34, 9027 (1986).

103. J. Humlicek, E. Schmidt, L. Bocanek, R. Svehla \& K. Ploog, "Exciton line-shapes of gaas/alas multiple-quantum wells," Phys. Rev. B 48, 5241 (1993).

104. M. Nakayama, "Theory of the excitonic polariton of the quantum well," Solid State Commun. 55, 1053 (1985).

105. F. Bassani, F. Tassone \& L. C. Andreani, "Excitons and polaritons in quantum wells," in Semiconductor superlattices and interfaces (North-Holland, Amsterdam ; New York, 1993).

106. D. V. O'Connor \& D. Phillips, "Time-correlated single-photon counting," (Academic Press, London ; Orlando, Fla., 1984).

107. R. K. Ahrenkiel, "Minority-carrier lifetime in iii-v semiconductors," in Semiconductors and semimetals 39, 39 (Academic Press Inc., Boston, 1993).

108. R. M. Ballew, J. N. Demas, N. P. Ayala, M. Grubb \& S. W. Snyder, "Computer interfaced fast kinetics luminescence decay experiment," J Chem Educ 68, 222 (1991).

109. R. M. Ballew \& J. N. Demas, "An error analysis of the rapid lifetime determination method for the evaluation of single exponential decays," Anal Chem 61, 30 (1989).

110. S. B. de-Leon \& B. Laikhtman, "Exciton-exciton interactions in quantum wells: Optical properties and energy and spin relaxation," Phys. Rev. B 6312, art. no. (2001).

111. T. Kamizato \& M. Matsuura, "Excitons in double quantum wells," Physical Review B 40, 8378 (1989).

112. C. C. Phillips, R. Eccleston \& S. R. Andrews, "Theoretical and experimental picosecond photoluminescence studies of the quantum-confined stark-effect in a strongly coupled double-quantum-well structure," Physical Review B 40, 9760 (1989).

113. A. M. Fox, D. A. B. Miller, G. Livescu et al., "Excitons in resonant coupling of quantum-wells," Phys. Rev. B 42, 1841 (1990). 
114. A. M. Fox, D. A. B. Miller, G. Livescu, J. E. Cunningham \& W. Y. Jan, "Excitonic effects in coupled quantum-wells," Phys. Rev. B 44, 6231 (1991).

115. M. M. Dignam \& J. E. Sipe, "Exciton-states in coupled double quantum-wells in a static electric-field," Phys. Rev. B 43, 4084 (1991).

116. Y. Takahashi, Y. Kato, S. S. Kano et al., "The effect of electric field on the excitonic states in coupled quantum well structures," Journal of Applied Physics 76, 2299 (1994).

117. S. de-Leon \& B. Laikhtman, "Exciton wave function, binding energy, and lifetime in inas/gasb coupled quantum wells," Physical Review B 61, 2874 (2000).

118. S. Y. Kim Ph.D. Thesis "Excitons in coupled double quantum wells" (University of California at Berkeley, 2001).

119. M. H. Szymanska \& P. B. Littlewood, "Excitonic binding in coupled quantum wells," Phys. Rev. B 67, art. no. 193305 (2003).

120. R. Strobel, R. Eccleston, J. Kuhl \& K. Kohler, "Measurement of the excitonformation time and the electron- tunneling and hole-tunneling times in a doublequantum-well structure," Phys. Rev. B 43, 12564 (1991).

121. A. Papoulis \& S. U. Pillai, "Probability, random variables, and stochastic processes," (McGraw-Hill, Dubuque, Iowa, 2002).

122. W. H. Press, "Numerical recipes in $\mathrm{c}^{++}$: The art of scientific computing," (Cambridge University Press, Cambridge, UK ; New York, 2002).

123. A. Esser, E. Runge, R. Zimmermann \& W. Langbein, "Photoluminescence and radiative lifetime of trions in gaas quantum wells," Phys. Rev. B 62, 8232 (2000).

124. A. Esser, R. Zimmermann \& E. Runge, "Theory of trion spectra in semiconductor nanostructures," Phys. Status Solidi B 227, 317 (2001).

125. N. N. Sibeldin, M. L. Skorikov \& V. A. Tsvetkov, "Formation of charged excitonic complexes in shallow quantum wells of undoped gaas/algaas structures under below-barrier and above-barrier photoexcitation," Nanotechnology 12, 591 (2001).

126. L. V. Butov, A. Imamoglu, K. L. Campman \& A. C. Gossard, "Coulomb effects in spatially separated electron and hole layers in coupled quantum wells (vol 92, pg 260, 2001)," J. Exp. Theor. Phys. 92, 752 (2001).

127. Y. Horikoshi, A. Fischer \& K. Ploog, "Photoluminescence quenching in reversebiased $a_{x} \mathrm{ga}_{1-\mathrm{x}}$ as/gaas quantum-well heterostructures due to carrier tunneling," Physical Review B 31, 7859 (1985).

128. T. Chavanapranee, S. Fujimoto \& Y. Horikoshi, "Photoluminescence quenching by optical bias in algaas/gaas single quantum wells," Japanese Journal of Applied Physics Part 1-Regular Papers Short Notes \& Review Papers 40, 6477 (2001).

129. H. Reinholz, "Mott effect for an electron-hole plasma in a two-dimensional structure," Solid State Commun. 123, 489 (2002).

130. S. Schmitt-Rink \& C. Ell, "Excitons and electron-hole plasma in quasi-2dimensional systems," J. Lumin. 30, 585 (1985).

131. S. F. Yoon, K. Radhakrishnan \& Q. H. Du, "Laser excitation induced photoluminescence linewidth reduction in molecular beam epitaxial inalas layers grown on inp substrates," Superlattice Microst. 23, 503 (1998). 
132. D. Yoshioka \& A. H. Macdonald, "Double quantum-well electron-hole systems in strong magnetic-fields," J. Phys. Soc. Jpn. 59, 4211 (1990).

133. L. V. Butov, A. Imamoglu, A. A. Shashkin et al., "Nonlinear photoluminescence kinetics of indirect excitons in coupled quantum wells," Phys. Status Solidi A 178, 83 (2000).

134. L. V. Butov, A. L. Ivanov, A. Imamoglu et al., "Stimulated scattering of indirect excitons in coupled quantum wells: Signature of a degenerate bose-gas of excitons," Phys. Rev. Lett. 86, 5608 (2001).

135. R. Eccleston, B. F. Feuerbacher, J. Kuhl, W. W. Ruhle \& K. Ploog, "Density dependent exciton radiative lifetimes in gaas quantum wells," Physical Review B 45, 11403 (1992).

136. M. Hirasawa \& T. Ishihara, "Exciton lifetime in quantum well with vicinal highdensity excitons," Physica E 7, 600 (2000).

137. A. L. Efros, C. Wetzel \& J. M. Worlock, "Effect of a random adiabtic potential on the optical-properties of 2-dimensional excitons," Phys. Rev. B 52, 8384 (1995).

138. D. S. Citrin, "Radiative lifetimes of excitons in quantum-wells - localization and phase-coherence effects," Phys. Rev. B 47, 3832 (1993).

139. M. Combescot \& C. Tanguy, "Reply to the comment by s. Rombouts et al. On "new criteria for bosonic behaviour of excitons"," Europhysics Letters 63, 787 (2003).

140. M. Colocci, M. Gurioli, A. Vinattieri et al., "Temperature dependence of exciton lifetimes in gaas/algaas quantum well structures," Europhysics Letters 12, 417 (1990).

141. M. Colocci, M. Gurioli \& A. Vinattieri, "Dynamics of carrier recombination in gaas/algaas quantum well structures," Physica Scripta T39, 211 (1991).

142. M. Colocci, M. Gurioli \& J. Martinezpastor, "Exciton relaxation dynamics in quantum well heterostructures," Journal De Physique IV 3, 3 (1993).

143. L. C. Andreani, F. Tassone \& F. Bassani, "Radiative lifetime of free excitons in quantum wells," Solid State Commun. 77, 641 (1991).

144. J. Martinez-pastor, A. Vinattieri, L. Carraresi et al., "Temperature dependence of exciton lifetimes in gaas/al ${ }_{x}$ ga $_{1-x}$ as single quantum wells," Phys. Rev. B 47, 10456 (1993).

145. J. Lee, E. S. Koteles \& M. O. Vassell, "Luminescence linewidths of excitons in gaas quantum-wells below 150-k," Phys. Rev. B 33, 5512 (1986).

146. V. Srinivas, J. Hryniewicz, Y. J. Chen \& C. E. C. Wood, "Intrinsic linewidths and radiative lifetimes of free-excitons in gaas quantum-wells," Phys. Rev. B 46, 10193 (1992).

147. D. S. Citrin, "Homogeneous-linewidth effects on radiative lifetimes of excitons in quantum-wells," Solid State Commun. 84, 281 (1992).

148. J. S. Blakemore, "Semiconducting and other major properties of gallium arsenide," Journal of Applied Physics 53, R123 (1982).

149. D. W. Snoke, V. Negoita \& K. Eberl, "Energy shifts of indirect excitons in coupled quantum wells," Journal of Luminescence 87-9, 157 (2000).

150. R. A. Abram, "Effects of heavy doping and high-excitation on the band-structure of gallium-arsenide," Semiconduct. Semimet. 39, 259 (1993). 
151. N. Peyghambarian, H. M. Gibbs, J. L. Jewell et al., "Blue shift of the exciton resonance due to exciton-exciton interactions in a multiple-quantum-well structure," Physical Review Letters 53, 2433 (1984).

152. D. Hulin, A. Mysyrowicz, A. Antonetti et al., "Well-size dependence of exciton blue shift in gaas multiple-quantum-well structures," Physical Review B 33, 4389 (1986).

153. L. V. Butov, A. A. Shashkin, V. T. Dolgopolov, K. L. Campman \& A. C. Gossard, "Magneto-optics of the spatially separated electron and hole layers in gaas/alxga1-xas coupled quantum wells," Phys. Rev. B 60, 8753 (1999).

154. V. Negoita, D. W. Snoke \& K. Eberl, "Huge density-dependent blueshift of indirect excitons in biased coupled quantum wells," Physical Review B 61, 2779 (2000).

155. K. Yang, "Dipolar excitons, spontaneous phase coherence, and superfluidinsulator transition in bilayer quantum hall systems at nu=1," Phys. Rev. Lett. 8705 (2001).

156. M. Baranov, L. Dobrek, K. Goral, L. Santos \& M. Lewenstein, "Ultracold dipolar gases - a challenge for experiments and theory," Phys. Scr. T102, 74 (2002).

157. G. M. Kavoulakis, "Bose-einstein condensation of indirect excitons in coupled quantum wells," J. Low. Temp. Phys. 132, 297 (2003).

158. L. J. Sham \& T. Ostreich, "Exciton interaction and spin dynamics in semiconductor heterostructures," J. Lumin. 87-9, 179 (2000).

159. E. D. Palik \& G. Ghosh, "Handbook of optical constants of solids," (Academic Press, San Diego, 1998).

160. I. Schnitzer, E. Yablonovitch, C. Caneau \& T. J. Gmitter, "Ultrahigh spontaneous emission quantum efficiency, 99.7-percent internally and 72-percent externally, from algaas/gaas/algaas double heterostructures," Applied Physics Letters 62, 131 (1993).

161. E. F. Schubert, "Light emitting diodes," (Cambridge University Press, Cambridge ; New York, 2003).

162. M. Colocci, M. Gurioli \& A. Vinattieri, "Relaxation dynamics in gaas $/ a l_{x} g a_{1-x} a s$ quantum well heterostructures," in Semiconductor superlattices and interfaces (North-Holland, Amsterdam ; New York, 1993).

163. A. Parlangeli, P. C. M. Christianen, J. C. Maan et al., "Optical observation of the energy-momentum dispersion of spatially indirect excitons," Phys. Rev. B 62, $15323(2000)$.

164. L. V. Butov, C. W. Lai, D. S. Chemla et al., "Observation of magnetically induced effective-mass enhancement of quasi-2d excitons," Phys. Rev. Lett. 87, art. no. 216804 (2001).

165. H. L. Stormer, D. C. Tsui \& A. C. Gossard, "The fractional quantum hall effect," Rev. Mod. Phys. 71, S298 (1999).

166. I. V. Lerner \& Y. E. Lozovik, "Correlation-energy and excitation-spectra of twodimensional electron-hole systems in high magnetic-fields," Solid State Commun. 36, 7 (1980).

167. D. Paquet, T. M. Rice \& K. Ueda, "Two-dimensional electron-hole fluid in a strong perpendicular magnetic-field - exciton bose condensate or maximum density two-dimensional droplet," Phys. Rev. B 32, 5208 (1985). 
168. N. E. Kaputkina \& Y. E. Lozovik, "Two-dimensional exciton with spatiallyseparated carriers in coupled quantum wells in external magnetic field," Physica E-Low-Dimensional Systems \& Nanostructures 12, 323 (2002).

169. A. Imamoglu, "Inhibition of spontaneous emission from quantum-well magnetoexcitons," Phys. Rev. B 54, 14285 (1996).

170. L. P. Gorkov \& Dzyalosh.Ie, "Contribution to theory of mott exciton in a strong magnetic field," Sov. Phys. JETP 26, 449 (1968).

171. A. A. Gorbatsevich \& I. V. Tokatly, "Formation of k-space indirect magnetoexcitons in double-quantum-well direct-gap heterostructures," Semicond Sci. Tech. 13, 288 (1998).

172. Y. E. Lozovik, I. V. Ovchinnikov, S. Y. Volkov, L. V. Butov \& D. S. Chemla, "Quasi-two-dimensional excitons in finite magnetic fields," Phys. Rev. B 65, art. no. 235304 (2002).

173. R. Eppenga, M. F. H. Schuurmans \& S. Colak, "New $k \cdot p$ theory for gaas/ga ${ }_{1-}$ ${ }_{x} a l_{x} a s$-type quantum wells," Phys. Rev. B 36, 1554 (1987).

174. G. Duggan, "Theory of heavy hole magnetoexcitons in gaas-(al,ga)as quantum well heterostructures," Phys. Rev. B 37, 2759 (1988).

175. J. M. Kosterliz \& D. J. Thouless, "Ordering, metastability and phase-transitions in 2 dimensional systems," J. Phys. C 6, 1181 (1973).

176. D. S. Petrov, M. Holzmann \& G. V. Shlyapnikov, "Bose-einstein condensation in quasi-2d trapped gases," Phys. Rev. Lett. 84, 2551 (2000).

177. R. K. Bhaduri, S. M. Reimann, S. Viefers, A. G. Choudhury \& M. K. Srivastava, "The effect of interactions on bose-einstein condensation in a quasi twodimensional harmonic trap," J. Phys. B 33, 3895 (2000).

178. U. Al Khawaja, J. O. Andersen, N. P. Proukakis \& H. T. C. Stoof, "Low dimensional bose gases," Phys. Rev. A 66, art. no. (2002).

179. J. P. Fernandez \& W. J. Mullin, "The two-dimensional bose-einstein condensate," J. Low. Temp. Phys. 128, 233 (2002).

180. A. Gorlitz, J. M. Vogels, A. E. Leanhardt et al., "Realization of bose-einstein condensates in lower dimensions," Phys. Rev. Lett. 8713, art. no. (2001).

181. L. V. Butov, L. S. Levitov, A. V. Mintsev et al., "Charge transport and phase transition in exciton rings," arXiv:cond (2003).

182. R. Rapaport, G. Chen, D. Snoke et al., "Mechanism of luminescence ring pattern formation in quantum well structures: Optically-induced in-plane charge separation," arXiv:cond (2003).

183. P. Bhattacharya, "Semiconductor optoelectronic devices," (Prentice Hall, Upper Saddle River, NJ, 1997).

184. D. V. Lang, R. A. Logan \& M. Jaros, "Trapping characteristics and a donorcomplex ( $d x)$ model for the persistent photoconductivity trapping center in tedoped al $\mathrm{ga}_{1-x}$ as," Physical Review B 19, 1015 (1979).

185. P. M. Mooney, "Deep donor levels (dx centers) in iii-v semiconductors," Journal of Applied Physics 67, R1 (1990).

186. L. Dobaczewski, P. Kaczor, Z. R. Zytkiewicz et al., "Hole capture at the $d x(s i)$ and $d x(t e)$ defects in $a_{x} g a_{1-x} a s, "$ Journal of Applied Physics 72, 3198 (1992).

187. G. Brunthaler, K. Ploog \& W. Jantsch, "Photoluminescence transients due to hole capture at dx centers in $a_{x} g a_{1-x} a s: S i, "$ Physical Review Letters 63, 2276 (1989). 
188. T. Sato \& T. Ishiwatari, "Deep levels in strongly si-compensated gaas and algaas," Journal of Applied Physics 91, 5158 (2002).

189. R. Piotrzkowski, E. Litwin-Staszewska, F. Bosc, J. Sicart \& J. L. Robert, "New type of persistent photoconductivity related to $d x$-center: The study of interband ppc in si-doped algaas," Physica B 274, 792 (1999).

190. H. Fujioka, E. R. Weber \& A. K. Verma, "Transient current study of lowtemperature-grown gaas using an n-i-n structure," Appl. Phys. Lett. 66, 2834 (1995).

191. A. K. Verma, J. Tu, J. S. Smith, H. Fujioka \& E. R. Weber, "Electrical characterization of low-temperature al0.3ga0.7as using n-i-n structures," Appl. Phys. Lett. 68, 699 (1996).

192. S. Ehret, H. Schneider, C. Schonbein, G. Bihlmann \& J. Fleissner, "Analysis of the transport mechanism in gaas/algaas quantum-well infrared photodetection structures using time resolved photocurrent measurements," Appl. Phys. Lett. 69, 931 (1996).

193. N. M. Haegel, C. R. Brennan \& A. M. White, "Transport in extrinsic photoconductors: A comprehensive model for transient response," J. Appl. Phys. 80, 1510 (1996).

194. D. C. Hutchings, C. B. Park \& A. Miller, "Modeling of cross-well carrier transport in a multiple quantum-well modulator," Appl. Phys. Lett. 59, 3009 (1991).

195. D. J. Moss, T. Ido \& H. Sano, "Calculation of photogenerated carrier escape rates from gaas $a l_{x} g a_{1-x}$ as quantum wells," IEEE J. Quantum. Elect. 30, 1015 (1994).

196. K. R. Lefebvre \& A. F. M. Anwar, "Electron escape time from single quantum wells," IEEE J. Quantum. Elect. 33, 187 (1997).

197. A. F. M. Anwar \& K. R. Lefebvre, "Electron escape via polar optical-phonon interaction and tunneling from biased quantum wells," Phys. Rev. B 57, 4584 (1998).

198. K. R. Lefebvre \& A. F. M. Anwar, "Electron-phonon interaction within an unbiased and biased quantum well," IEEE J. Quantum. Elect. 35, 216 (1999).

199. N. Balkan, B. K. Ridley \& A. J. Vickers, "Negative differential resistance and instabilities in 2-d semiconductors," (Plenum Press, New York, 1993).

200. Z. S. Gribnikov, K. Hess \& G. A. Kosinovsky, "Nonlocal and nonlinear transport in semiconductors - real-space transfer effects," J. Appl. Phys. 77, 1337 (1995).

201. R. K. Willardson \& A. C. Beer, "Injection phenomena," (Academic Press, New York, 1970).

202. I. H. Tan, G. L. Snider, L. D. Chang \& E. L. Hu, "A self-consistent solution of schrodinger-poisson equations using a nonuniform mesh," J. Appl. Phys. 68, 4071 (1990).

203. E. Schöll, "Nonlinear spatio-temporal dynamics and chaos in semiconductors," (Cambridge University Press, Cambridge ; New York, 2001).

204. N. Peyghambarian, L. L. Chase \& A. Mysyrowicz, "Bose-einstein statistical properties and condensation of excitonic molecules in cucl," Physical Review B 27, 2325 (1983). 
205. L. L. Chase, N. Peyghambarian, G. Grynberg \& A. Mysyrowicz, "Evidence for bose-einstein condensation of biexcitons in cucl," Physical Review Letters 42, 1231 (1979).

206. M. Hasuo, N. Nagasawa, T. Itoh \& A. Mysyrowicz, "Bose-einstein condensation of biexciton system in cucl," Journal of Luminescence 60-1, 758 (1994).

207. D. W. Snoke, J. P. Wolfe \& A. Mysyrowicz, "Evidence for bose-einstein condensation of excitons in $\mathrm{Cu}_{2} \mathrm{O}$," Physical Review B 41, 11171 (1990).

208. J. L. Lin \& J. P. Wolfe, "Bose-einstein condensation of paraexcitons in stressed $\mathrm{Cu}_{2} \mathrm{O}, "$ Physical Review Letters 71, 1222 (1993).

209. A. Mysyrowicz, E. Benson \& E. Fortin, "Directed beam of excitons produced by stimulated scattering," Physical Review Letters 77, 896 (1996).

210. S. Pau, H. Cao, J. Jacobson et al., "Observation of a laserlike transition in a microcavity exciton polariton system," Physical Review A 54, R1789 (1996).

211. E. Benson, E. Fortin \& A. Mysyrowicz, "Anomalous exciton transport in cu2o: Excitonic superfluidity or phonon-wind effect?," Solid State Commun. 101, 313 (1997).

212. K. E. O'Hara \& J. P. Wolfe, "Relaxation kinetics of excitons in cuprous oxide," Phys. Rev. B 62, 12909 (2000).

213. A. Jolk, M. Jorger \& C. Klingshirn, "Exciton lifetime, auger recombination, and exciton transport by calibrated differential absorption spectroscopy in cu2o," Phys. Rev. B 65, art. no. (2002).

214. S. D. Baranovskii, R. Eichmann \& P. Thomas, "Temperature-dependent exciton luminescence in coupled quantum wells," Physica Status Solidi B-Basic Research 205, R19 (1998).

215. S. D. Baranovskii \& P. Thomas, "Phase-transition of an exciton system in gaas coupled quantum-wells and on fermi-dirac distribution of excitons in coupled quantum-wells - comment," Phys. Rev. Lett. 69, 993 (1992).

216. S. D. Baranovskii, R. Eichmann \& P. Thomas, "Temperature-dependent exciton luminescence in quantum wells by computer simulation," Phys. Rev. B 58, 13081 (1998).

217. L. V. Butov, A. Zrenner, G. Abstreiter, G. Bohm \& G. Weimann, "Condensation of indirect excitons in coupled alas/gaas quantum-wells," Phys. Rev. Lett. 73, 304 (1994).

218. A. V. Larionov \& V. B. Timofeev, "Condensation of interwell excitons in gaas/algaas double quantum wells," JETP Lett. 73, 301 (2001).

219. A. A. Dremin, V. B. Timofeev, A. V. Larionov, J. Hvam \& K. Soerensen, "Phase diagram of the bose condensation of interwell excitons in gaas/algaas double quantum wells," JETP Lett. 76, 450 (2002).

$220 . \quad$ R. J. Glauber, "Coherent and incoherent states of radiation field," Physical Review 131, 2766 (1963).

221. J. J. Slosser \& P. Meystre, "Resource letter: Cqo-1: Coherence in quantum optics," American Journal of Physics 65, 275 (1997).

222. B. Laikhtman, "Coherence of the exciton condensate luminescence," Europhysics Letters 43, 53 (1998). 
223. A. Olaya-Castro, F. J. Rodriguez, L. Quiroga \& C. Tejedor, "Restrictions on the coherence of the ultrafast optical emission from an electron-hole-pair condensate," Physical Review Letters 8724 (2001).

224. Y. E. Lozovik \& I. V. Ovchinnikov, "Many-photon coherence of bose-condensed excitons: Luminescence and related nonlinear optical phenomena," Phys. Rev. B 66, art. no. (2002).

225. D. Snoke, "Spontaneous bose coherence of excitons and polaritons," Science 298, 1368 (2002).

226. A. Olaya-Castro, L. Quiroga \& C. Tejedor, "Coherence properties of a radiating electron-hole condensate," Solid State Commun. 127, 141 (2003).

227. L. Mandel \& E. Wolf, "Optical coherence and quantum optics," (Cambridge University Press, Cambridge ; New York, 1995).

228. U. Leonhardt \& H. Paul, "Measuring the quantum state of light," Progress in Quantum Electronics 19, 89 (1995).

229. C. Iaconis \& I. A. Walmsley, "Direct measurement of the two-point field correlation function," Optics Letters 21, 1783 (1996).

230. S. Haacke, S. Schaer, B. Deveaud \& V. Savona, "Interferometric analysis of resonant rayleigh scattering from two-dimensional excitons," Phys. Rev. B 61, R5109 (2000).

231. S. Haacke, "Resonant rayleigh scattering by wannier excitons in a twodimensional disordered potential," Reports on Progress in Physics 64, 737 (2001).

232. T. Ostreich, T. Portengen \& L. J. Sham, "Second-order optical response from a bose-einstein condensate of excitons," Solid State Communications 100, 325 (1996).

233. J. A. Leegwater \& S. Mukamel, "Four-wave mixing signatures of exciton bose condensation," Chemical Physics Letters 217, 456 (1994).

234. J. A. Leegwater \& S. Mukamel, "Transient grating spectroscopy of exciton sound-waves in dense exciton fluids," Chemical Physics Letters 203, 125 (1993).

235. Y. E. Lozovik \& I. V. Ovchinnikov, "Stimulated light backscattering from exciton bose condensate," JETP Lett. 74, 288 (2001).

236. Y. E. Lozovik, I. L. Kurbakov \& I. V. Ovchinnikov, "Nonlinear optical phenomena in coherent phase of $2 d$ exciton system," Solid State Communications 126, 269 (2003).

237. H. Yoon, A. L. Gutierrezaitken, R. Jambunathan, J. Singh \& P. K. Bhattacharya, "A cold inp-based tunneling injection-laser with greatly reduced auger recombination and temperature-dependence," Photonics Tech. Lett. 7, 974 (1995).

238. A. Parlangeli, P. C. M. Christianen, J. C. Maan \& M. Henini, "Bistability and discontinuity in the tunnel current of two-dimensional electron-hole layers," Physical Review B 63, 115307 (2001). 